\title{
CHEMICAL SCIENCES DIVISION Annual Report 1994
} June 1995

Lawrence Berkeley Laboratory

University of California Berkeley, California 94720

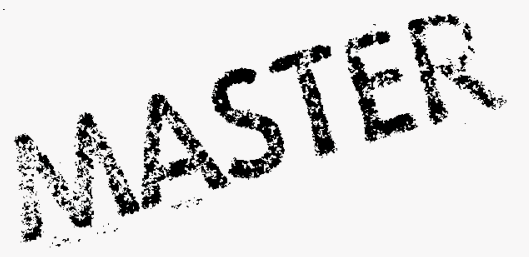


8 monscispeper 


\section{DISCLAIMER}

This report was prepared as an account of work sponsored by an agency of the United States Government. Neither the United States Government nor any agency thereof, nor any of their employees, make any warranty, express or implied, or assumes any legal liability or responsibility for the accuracy, completeness, or usefulness of any information, apparatus, product, or process disclosed, or represents that its use would not infringe privately owned rights. Reference herein to any specific commercial product, process, or service by trade name, trademark, manufacturer, or otherwise does not necessarily constitute or imply its endorsement, recommendation, or favoring by the United States Government or any agency thereof. The views and opinions of authors expressed herein do not necessarily state or reflect those of the United States Government or any agency thereof. 


\section{DISCLAIMER}

Portions of this document may be illegible in electronic image products. Images are produced from the best available original document. 


\section{Contents}

\section{REMARKS BY THE DIVISION DIRECTOR}

Charles B. Harris

\section{CHEMICAL SCIENCES}

Fundamental Interactions

Chemical Physics

Energy Transfer and Structural Studies of Molecules on Surfaces

Charles B. Harris, Investigator

Photochemistry of Materials in the Stratosphere

Harold S. Johnston, Investigator.

Laser Sources and Techniques

Andrew H. Kung, Investigator

Crossed Molecular Beams

Yuan T. Lee and Arthur Suits, Investigators 8

Molecular Interactions

William A. Lester, Jr., Investigator.

Theory of Atomic and Molecular Collision Processes

William H. Miller, Investigator

Selective Photochemistry

C. Bradley Moore, Investigator

Photodissociation of Free Radicals

Daniel M. Neumark, Investigator

Physical Chemistry with Emphasis on Thermodynamic Properties

Kenneth S. Pitzer, Investigator

Atomic Physics

Search for the Electric Dipole Moment of the Electron

Eugene D. Commins, Investigator

High-Energy Atomic Physics

Harvey Gould, Investigator

Atomic Physics

Michael H. Prior, Investigator

Processes and Techniques

Chemical Energy

High-Energy Oxidizers and Delocalized-Electron Solids

Neil Bartlett, Investigator. 
Catalytic Conversion of $\mathrm{C}_{1}$ Compounds

Alexis T. Bell, Investigator 44

Transition-Metal-Catalyzed Conversion of $\mathrm{CO}, \mathrm{NO}, \mathrm{H}_{2}$ and Organic

Molecules to Fuels and Petrochemicals

Robert G. Bergman, Investigator 48

New Molecular Precursors for Low-Temperature Routes to Oxide Materials

T. Don Tilley, Investigator.

Potentially Catalytic and Conducting Polyorganometallics

K. Peter C. Vollhardt, Investigator.

Heavy-Element Chemistry

Actinide Chemistry

Norman M. Edelstein, Richard A. Andersen,

Kenneth N. Raymond, and David K. Shuh, Investigators

Chemical Engineering Sciences

Molecular Thermodynamics for Phase Equilibria in Mixtures

John M. Prausnitz, Investigator

\section{LABORATORY-DIRECTED RESEARCH AND DEVELOPMENT (LDRD)}

Exploration of the Interface Between Homogeneous and Heterogeneous Catalysis:

Synthesis and Study of Organometallic Catalysts Supported on Novel Polysiloxane

Materials with Controllable Solubility Properties

Robert G. Bergman and Bruce M. Novak, Investigators.

Ultrafast Dynamics of Electrons at Surfaces

Charles B. Harris, Investigator

Structure and Chemistry of Nonmetallic Adsorbates at Semiconductor Interfaces Investigated by Synchrotron Radiation Techniques

David K. Shuh, Investigator

WORK FOR OTHERS

United States Office of Naval Research

Superconducting Properties of High- $T_{\mathrm{c}}$ Oxides

Vladimir Z Kresin, Investigator.

\section{APPENDICES}

Appendix A: Division Personnel

Appendix B: Index of Investigators 


\section{REMARKS BY THE DIVISION DIRECTOR}

The Chemical Sciences Division (CSD) is one of ten research Divisions of the Lawrence Berkeley Laboratory, a Department of Energy National Laboratory. This Division is composed of individual research groups that are organized into five scientific areas: Chemical Physics, Inorganic/Organometallic Chemistry, Actinide Chemistry, Atomic Physics, and Chemical Engineering. Our research staff continues to achieve fundamental advances in understanding the structure and reactivity of critical reaction intermediates and transients and the dynamics of elementary chemical reactions. Significant advances in the theoretical understanding of these basic processes have also been made. In addition, the Division supports a strong effort in heterogeneous and homogeneous catalysis.

The CSD is unique in that a good deal of our Division's research activity occurs in laboratories located on the campus of the University of California at Berkeley, involving students and faculty in the College of Chemistry. Several large programs, notably the Actinide Chemistry Program and the Atomic Physics Program, are based at the Lawrence Berkeley Laboratory (LBL) site just east of and above the University campus.

The benefits of close ties with the campus are broad and numerous. One of the most important of these is the vigor, dedication, and high productivity that graduate student research assistants bring to our various research projects. The Laboratory has a staff of highly skilled engineers and crafts technicians who support the scientific mission, and it is the site of several major, world-class user facilities such as the Advanced Light Source (ALS). Combined, the two institutions represent a highly enriched research environment, attracting a large number of eminent scientists and leaders from all over the worldmany of whom have close associations with the Chemical Sciences research programs.

In FY 1994 the CSD neared completion of developing two end-stations and a beamline to advance chemical dynamics research at the ALS. In support of DOE's national role in combustion research and chemical science, the beamline effort will enable researchers from around the world to make fundamental advances in understanding the structure and reactivity of critical reaction intermediates and transients, and in understanding the dynamics of elementary chemical reactions. The Chemical Dynamics Beamline initiative at the ALS envisions both experimental and theoretical efforts that would contribute to accurate and reliable models for predicting combustion properties to improve the design of engines, burners, and other combustion devices.

Awards and honors received by CSD investigators in FY 1994 include:

- Neil Bartlett was the George Cady Lecturer at the University of Washington.

- Robert G. Bergman received the U.S. Department of Energy E.O. Lawrence Award in Chemistry.

- Alexis T. Bell was a Reilly Lecturer at the University of Notre Dame, and a Hoyt C. Hottel Lecturer at the Massachusetts Institute of Technology.

- Charles B. Harris was made a Fellow of the Optical Society of America.

- Yuan T. Lee received an Honorary Doctor of Science Degree (Honoris Causa) from the Cheng Kung University and the Hong Kong Baptist University.

- William Miller was made a Fellow of the American Academy of Arts and Sciences and Honorary Professor at Shandong University, People's Republic of China. He received the American Chemical Society Award in Theoretical Chemistry and the Christensen Visiting Fellowship from St. Catherine's College in Oxford.

- C.B. Moore received the Earle K. Plyler Prize of the American Physical Society.

- Daniel M. Neumark was made a Fellow of the American Physical Society.

- John M. Prausnitz was Dexter Baker Lecturer at Lehigh University, and Plenary Lecturer at the 100th Birthday of the Bunsengesellschaft für Phys. Chemie Christensen Fellow, St. Catherine's College, Oxford University.
Charles B. Harris

Division Director

Chemical Sciences Division 
Chemical Sciences 


\section{FUNDAMENTAL INTERACTIONS}

\section{CHEMICAL PHYSICS}

\section{Energy Transfer and Structural Studies of Molecules on Surfaces*}

\author{
Charles B. Harris, Investigator
}

\section{INTRODUCTION}

The goal of this research is to understand the dynamics of excited electronic states on surfaces, at interfaces, and in condensed phases. The research program is both theoretical and experimental in character. It includes nonlinear optical and ultrafast laser techniques in the picosecond to femtosecond regime, in addition to more conventional surface science instruments for characterizing surfaces and adsorbate-surface interactions. Recent work has focused on the application of the new techniques that we have developed to probe the dynamics of electrons at interfaces and carrier diffusion in semiconductors. The results of this program have a direct bearing on the properties of nanoscale and quantum electronic devices, optoelectronic materials, and on other problems of general interest such as the dynamics of electrical transmission in conductors on ultrafast timescales and the optical properties of thin films.

\section{Electronic Dynamics at Metal-Insulator Interfaces (Publications 1,2)}

\section{C.B. Harris, R.E. Jordan, R.L. Lingle, Jr., J.D. McNeill, and N.-H. Ge}

New results for electron dynamics at interfaces and in thin films are emerging from a combination of ultrafast lasers and electron spectroscopy. Time- and angle-resolved two-photon photoemission (TPPE) spectroscopy probes the band structure and femtosecond dynamics of excited electronic states at surfaces and interfaces. Electrons excited just below the photoemission threshold of a metal may remain bound at the metal surface by the image potential. We have found that these image potential states persist in the presence of adsorbed molecules. Electrons in the image potential states, localized in the microscopic

*This work was supported by the Director, Office of Energy Research, Office of Basic Energy Sciences, Chemical Sciences Division, of the U.S. Department of Energy under Contract No. DE-AC03-76SF00098. region just outside the surface and interacting strongly with adsorbed molecules, provide a unique probe of the potential at the metal-insulator interface and of the dynamics of the electron in atomically thin films.

Major strides have been made in the study of rare gas films on metal substrates. These experiments show that angle-resolved TPPE can serve as a powerful and general probe of the transition at an interface from two to three dimensions. We have performed layer-resolved twophoton photoelectron spectroscopy of the conduction band of the $\mathrm{Xe} / \mathrm{Ag}(111)$ interface for up to nine atomic layers of Xe. For multilayers of $\mathrm{Xe} / \mathrm{Ag}(111)$, TPPE shows how the 2-D states of the interface evolve toward the 3-D bulk states of crystalline $\mathrm{Xe}$, and the corresponding effective mass measurement by angle-resolved TPPE tracks the spatial extent of the electron as a function of layer thickness. The binding energies of the three lowest quantum states of the conduction band are plotted as a function of layer thickness in Figure 1-1. The $n=1,2$, and 3 bare surface image

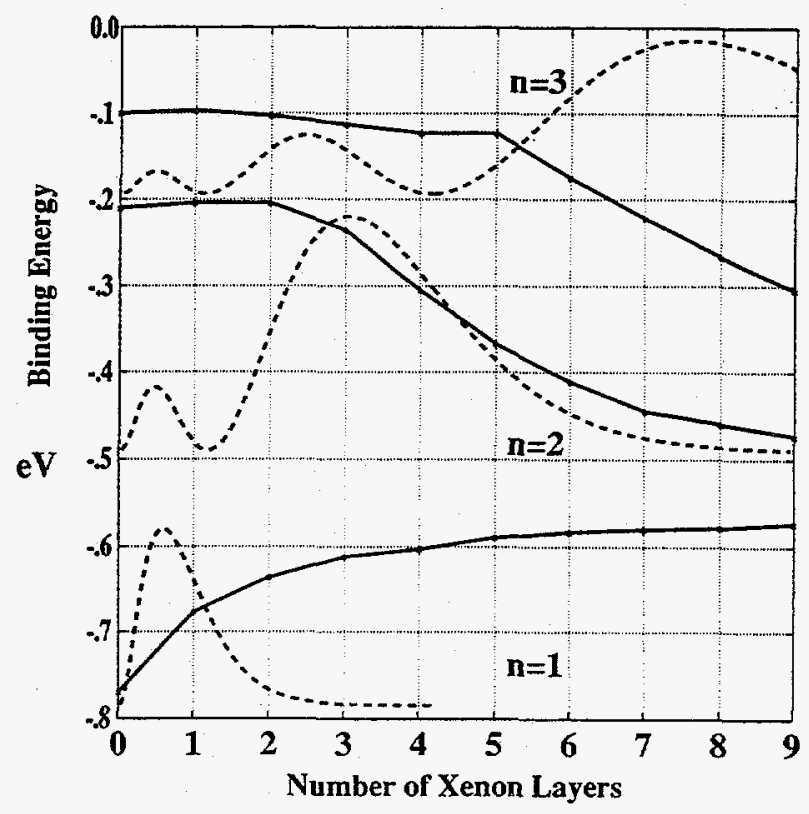

Figure 1-1. The solid lines are the experimental binding energies of an excess electron at the $\mathrm{Xe} / \mathrm{Ag}(111)$ interface for the layer thicknesses indicated. One layer of Xe is approximately $4.1 \AA$ thick. The dashed lines are hydrogenic image potential wave functions for a bare metal surface drawn to scale with layer thickness. This illustrates that a particular image potential state, labeled by its principal quantum number $n$, does not respond to the presence of the adlayer until the layer substantially overlaps with the electron density. 
potential state wave functions are plotted to scale, showing that the energy of a particular state is only perturbed when the layer becomes thick enough to spatially overlap with the electron density distribution of that quantum state.

We have recently solved a long-standing puzzle concerning the effect of insulating monolayers on the electrostatic fields near a metal surface. In previous work, ${ }^{1}$ we found that excess electron binding energies on molecular monolayers (including xenon, cyclohexane, and n-octane) could be explained without invoking the dielectric response of the monolayer. A systematic study of n-alkanes on $\mathrm{Ag}(111)$ has now revealed that the binding energy of the image potential electron at the monolayer is indeed correlated with both the molecular polarizability and the dielectric constant of the associated bulk material, as shown in Figure 1-2.

A classical electrostatic continuum model indicates that the potential for the metal-insulator interface consists of two principal terms: one is the screened image potential interaction between the electron and the metal while the second is the direct, attractive polarization interaction between the electron and the adlayer. In fact, the attractive interaction between the adlayer and the image electron dominates the screening effect for monolayers with high dielectric susceptibility. Thus a monolayer may either increase or decrease the binding of an image potential electron to the surface. The two effects were roughly in balance for the previously studied monolayers composed of xenon, cyclohexane, and n-octane.

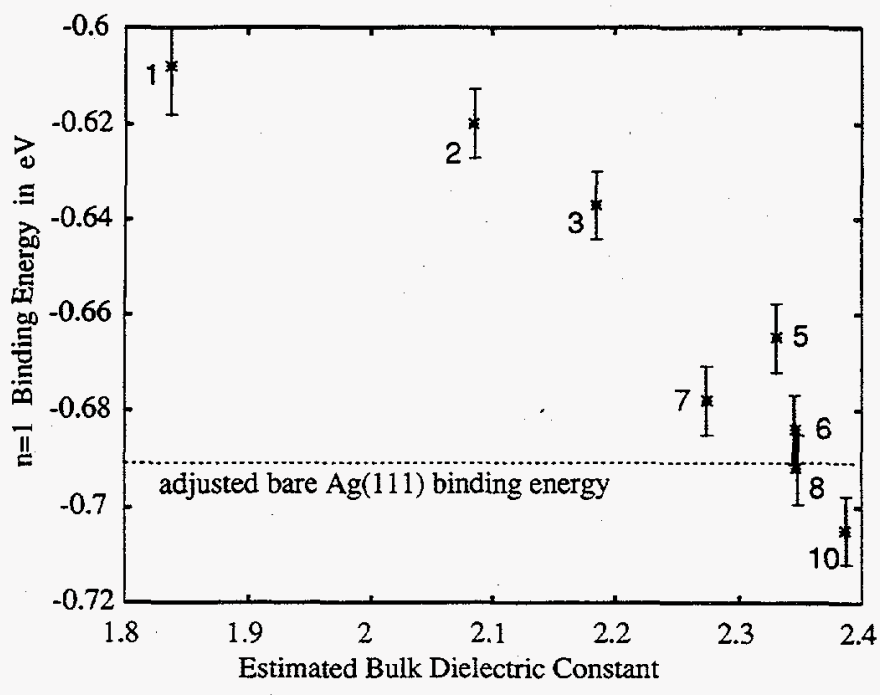

Figure 1-2. Plot of $n=1$ image potential electron binding energy in the presence of monolayers of various $n$-alkanes on $\mathrm{Ag}(111)$. The $\mathrm{x}$-axis is the estimated dielectric constant for the associated bulk crystalline material. As the dielectric susceptibility of the layer increases, the binding of the excess electron to the layer dominates screening of the electronmetal interaction, leading to stronger net binding.
Finally, we have continued the study of 2-D localization of electrons at metal-alkane interfaces by confirming the predictions of the model we initially applied to this problem in Publication 1. We have observed localized electrons in the bilayer of ethane, n-propane, and $\mathrm{n}$-decane. The ethane case is particularly important, since the theory we applied in Publication 1 predicts that ethane layers would be the shortest alkane to show localization at the interface. We have now begun to investigate localization phenomena at interfaces with alkene and aromatic layers.

1. W.R. Merry, R.E. Jordan, D.F. Padowitz, and C.B. Harris, Surf. Sci. 295, 393 (1993).

\section{Ultrafast Dynamics in Solution ${ }^{\dagger}$}

\author{
C.B Harris, M.C. Asplund, S.E. Bromberg, J.C. King, \\ T. Lian, and C.T. Vala
}

Chemical reactions are strongly influenced by the interaction of solutes with their surrounding solvents. These interactions can stabilize transition states, change energy level ordering, or alter the flow of energy from one mode to another. One way to study these interactions is to look at the rate at which a solute is able to transfer energy from its vibrational modes to the solvent energy modes. Past studies by this group showed that vibrationally hot $I_{2}$ cooled with a time constant of 140 picoseconds (ps), and that the rate of cooling depends on the density of solvent states at the $\mathrm{I}_{2}$ oscillator frequency.

Recent work by Barbara and coworkers and Ruhman and coworkers has shown that $\mathrm{I}_{2}{ }^{-}$in polar solvents vibrationally cools in 2-4 ps, much faster than $I_{2}$ in nonpolar solvents. This effect could be due to two factors. $\mathrm{I}_{2}{ }^{-}$has a weaker bond, and would therefore have a different overlap with the solvent modes. $\mathrm{I}_{2}{ }^{-}$also has a charge that can interact with a dipole in the solvent. In order to better understand this system, we have studied the vibrational cooling of $\mathrm{I}_{2}{ }^{-}$in nonpolar solvents. This provides cooling that should be independent of Coulombic effects, and therefore analogous to the $I_{2}$ system. Our results show that $\mathrm{I}_{2}-$ in $\mathrm{CCl}_{4}$ vibrationally cools with a timescale of 4-5 ps, approximately the same timescale as in polar solvents.

Other work in the area of transition metal complexes has focused on understanding the reaction mechanism of carbon-hydrogen bond activation. When a complex such as $\mathrm{Cp} * \operatorname{Ir}(\mathrm{CO})_{2}\left(\mathrm{Cp}^{*}=\eta 5-\mathrm{C}_{5} \mathrm{Me}_{5}\right)$ is irradiated with $\mathrm{UV}$ light in alkane solution, it reacts with the solvent, losing $\mathrm{CO}$ to form $\mathrm{Cp}^{*} \operatorname{IrCO}(\mathrm{R})(\mathrm{H})$. This reaction is important because it represents the first step in a potential catalytic conversion of unreactive alkanes into more useful molecules. While much has been learned about the reaction since its 
discovery in 1982, studies aimed at directly observing the intermediates in the reaction have been hindered by the rapid rate of reaction and the low quantum yield.

In an effort to understand the dynamics responsible for the low quantum yield, we have carried out picosecond transient absorption experiments on $\mathrm{Cp}^{*} \operatorname{Ir}(\mathrm{CO})_{2}$ in room temperature cyclohexane and its congener, $\mathrm{Cp} * \mathrm{Rh}(\mathrm{CO})_{2}$, in room temperature pentane. Our results indicate that, following absorption of a photon, most of the molecules promoted to an excited electronic state decay directly back to the ground state. We found that this process occurs very quickly, taking $30-40$ ps. We therefore conclude that the quantum yield is low because most of the excited molecules are returning to the ground state before reacting with the solvent.

$\varlimsup^{\dagger}$ National Science Foundation Program using DOE equipment.

\section{Work in Progress}

The local potential fluctuations in the alloy semiconductor $\mathrm{CdS}_{\mathrm{x}} \mathrm{Se}_{1-\mathrm{x}}$ have been examined via their effects on the carrier dynamics as probed by ultrafast fluorescence upconversion. Such substitutional alloys are an intermediate case between well-ordered pure crystals and highly disordered glassy semiconductors. This disorder is a result of local potential fluctuations arising from the random occupation of the sulfur-selenium sites and gives rise to localized states just below the band edge. Because the localized states have differing lifetimes that vary as a function of their localization energy, their interaction with the delocalized states in the conduction band causes the fluorescence to change its form from a single exponential decay (observed in pure crystals) to that of a "stretched" exponential, also known as a Kohlrausch function. This functional form is an exponential with the exponent raised to a power between zero and one. The measured decays in these experiments display "stretch" exponents ranging from around 0.5 for the most disordered samples to unity for pure CdSe and CdS. Since the most disordered crystals, those with $\mathrm{x}=0.5$, have the greatest number of localized states distributed over the widest range of localization energies, these results validate the model given above for coupling between localized and delocalized states.

Examination of the signal rise times can provide further information on the details of these interactions. In addition to revealing the effects of the localized states on intraband relaxation, the rise times probe diffusion of carriers through samples with graded composition, since the band gap varies in a well-characterized manner with depth. At the present time, the measured rise times in fixed composition alloys are on the order of the instrumental resolution, which is currently 1 ps with our cavity-dumped dye laser synchronously pumped by a mode-locked $\mathrm{Nd}$ :YLF laser. To fully resolve these rise times, a regeneratively amplified $100 \mathrm{fs}$ Ti:sapphire system will be employed.

The work on electron dynamics at surfaces and interfaces is taking new directions due to major improvements being made in our apparatus. The incorporation of an amplified Ti:sapphire laser will improve our time resolution to $100 \mathrm{fs}$, while utilization of an optical parametric amplifier will give us widely tunable ultraviolet pulses. Time-resolved studies of electron behavior at metal-polymer interfaces have begun, and a new program of study focusing on magnetic films and metal-semiconductor junctions is being initiated in FY 1995. The study of magnetic films will test theories of 2-D magnetism and probe the magnetic properties of nanometer-scale metal-metal structures.

\section{FY 1994 PUBLICATIONS AND REPORTS}

\section{Refereed Journals}

1. R.L. Lingle, Jr., D.F. Padowitz, R.E. Jordan, J.D. McNeill, and C.B. Harris, "Two-Dimensional Localization of Electrons at Interfaces," Phys. Rev. Lett. 72, 2243 (1994).

\section{Other Publications}

2. D.F. Padowitz, C.B. Harris, R.E. Jordan, R.L. Lingle, Jr., J.D. McNeill, and W.R. Merry, "Two-photon Photoemission and the Dynamics of Electrons at Interfaces," SPIE Proceedings: Laser Techniques for Surface Science, Vol. 2125 (1994).

\section{Invited Talks}

3. C.B. Harris, "The Properties and Dynamics of Electrons at Interfaces," Solvent Dynamics Effects on Relaxation Phenomena Conference in Okazaki, Japan, October 1993.

4. C.B. Harris, "The Properties and Dynamics of Electrons at Interfaces," Symposium on Molecular Structure, Hiroshima University, Japan, October 8-12, 1993.

5. C.B. Harris, "Direct Femtosecond Measurements of SingleCollision Dominated Geminate Recombination Times of Molecules in Solution," American Chemical Society Pacific Conference on Chemistry and Spectroscopy, Pasadena, CA, October 14-23, 1993.

6. C.B. Harris, "The Properties and Dynamics of Electrons at Interfaces," El-Sayed Symposium, University of California, Los Angeles, CA, November 5, 1993.

7. C.B. Harris, "The Properties and Dynamics of Electrons at Interfaces," Department of Chemistry, University of Victoria, Canada, November 18, 1993. 
8. C.B. Harris, "The Properties and Dynamics of Electrons at Interfaces," American Physical Society Meeting, Crystal City, VA, April 18-22, 1994.

9. J.C. King, "Vibrational Relaxation of a Charged Species in a Nonpolar Solvent," Ultrafast Phenomena IX Conference, Dana Point, CA, May 4-8, 1994.

10. R.L. Lingle, Jr., "Behavior of Electrons at Nanometer-Scale Metal Dielectric Interfaces," Center for Naval Analyses, Alexandria, VA, May 19, 1994.

11. C.B. Harris, "The Properties and Dynamics of Electrons at Interfaces," Conference on Ultrafast Processes in Chemistry and Biology, Umea, Sweden, June 13-16, 1994.
12. R.L. Lingle, Jr., "Behavior of Electrons at Nanometer-Scale Metal Dielectric Interfaces," Hewlett-Packard Laboratories, Palo Alto, CA, June 23, 1994.

13. C.B. Harris, "The Properties and Dynamics of Electrons at Interfaces," Lasers in Surface Science, International Centre for Theoretical Physics, Adriatco Research Conference, Trieste, Italy, August 9-12, 1994.

14. C.B. Harris, "Localization of Electrons in Restricted Geometries," 208th National American Chemical Society Meeting, Washington, D.C., August 21-26, 1994.

15. J. C. King, "Watching Chemistry as It Happens: Ultrafast Spectroscopy," Department of Chemistry, Westmont College, Santa Barbara, CA, August 31, 1994. 


\section{Photochemistry of Materials in the Stratosphere*}

\author{
Harold S. Johnston, Investigator
}

\section{INTRODUCTION}

This research is concerned with global change in the chemistry of the atmosphere, including theoretical and interpretative gas-phase and heterogeneous photochemistry.

\section{Atmospheric Dynamics and Photochemistry (Publications 1 and 2)}

This project, in collaboration with Lawrence Livermore National Laboratory, carried out a critical evaluation of 11 multidimensional atmospheric models with respect to their formulation of atmospheric motions and particulate settling (Publication 1). The observations [U.S. Atomic Energy Commission (AEC)] of gaseous carbon-14 and of particulate strontium-90 in the atmosphere from 1963 to 1970 provide unique, but limited, data up to an altitude of about $35 \mathrm{~km}$ for testing long-term atmospheric motions. Model calculations for carbon- 14 begin for conditions as they were on October 1963, nine months after the conclusion of the nuclear bomb tests. The initial conditions for the calculations are derived by three methods. The measured carbon- 14 vertical profiles at $31 \mathrm{~N}$ are qualitatively different from all of the models; the measured vertical profiles show a maximum mixing ratio in the altitude range 20 to $25 \mathrm{~km}$ from October 1963 through July 1966, but all modeled profiles show mixing ratio maxima that increase in altitude from $20 \mathrm{~km}$ in October 1963 to greater than $40 \mathrm{~km}$ by April 1966. Both carbon- 14 and strontium-90 data indicate that the models differ substantially among themselves with respect to stratosphere-troposphere exchange rate. Strontium-90 data indicate that aerosol settling is important up to at least 35 $\mathrm{km}$ altitude. Relative to the measurements, about three- quarters of the models transport carbon-14 from the lower stratosphere to the troposphere too rapidly, and all models with all three sets of initial conditions appear to sweep carbon-14 out of the mid-stratosphere (above $28 \mathrm{~km}$ ) much more slowly than that observed from 1963 to 1966 to 1970 .

Publication 2 is a review of recent calculations concerning the effect of a large proposed fleet of stratospheric aircraft on the vertical ozone column. Between 1981 to 1991 , atmospheric models predicted a large ozone reduction for such aircraft cruising at an altitude of $20 \mathrm{~km}$, but they also predicted a strong sensitivity to flight altitude with little of no ozone change for flight between 15 and $17 \mathrm{~km}$. Calculations, for the first time in 1991 including heterogeneous chemistry on stratospheric sulfuric acid aerosols, show little of no ozone change for flight up to $20 \mathrm{~km}$. These results emphasize the need for complete understanding of heterogeneous chemistry in the stratosphere.

\section{FY 1994 PUBLICATIONS AND REPORTS}

\section{Refereed Journals}

1. D.E. Kinnison, H.S. Johnston, and D.J. Wuebbles, "Model Study of Atmospheric Transport Using Carbon 14 and Strontium 90 as Inert Tracers," J. Geophys. Res. 99, 20647 (1994); LBL-36626.

\section{Other Publications}

2. H.S. Johnston, "Stratospheric Aircraft: Impact on the Stratosphere?" CHEMRAWN VII, A World Conference, The Chemistry of the Atmosphere: Its Impact on Global Change, edited by J.G. Calvert (Blackwell Scientific Publications, 1994); LBL-31884.

\section{Invited Talks}

3. H.S. Johnston, "Two Ozone Holes Found in 1985," Emory University, Atlanta, GA, April 1994 and Naval Research Laboratory, June 1994.

\footnotetext{
*This work was supported by the Director, Office of Energy Research, Office of Basic Energy Sciences, Chemical Sciences Division of the U.S. Department of Energy under Contract No. DE-AC03-76SF00098.
} 


\section{Laser Sources and Techniques*}

Andrew H. Kung, Investigator

\section{INTRODUCTION}

This program focuses on the development of novel laser and spectroscopic techniques in the IR, UV, and VUV regions for studying combustion-related molecular dynamics at the microscopic level. Laser spectroscopic techniques have proven to be extremely powerful in the investigation of molecular processes that require very high sensitivity and selectivity. Our approach is to use quantum electronic and nonlinear optical techniques to extend the spectral coverage and to enhance the optical power of ultrahigh resolution laser sources so as to obtain photoionization, fluorescence, and photoelectron spectra of jet-cooled free radicals and to analyze products resulting from unimolecular and bimolecular reactions. New spectroscopic techniques are developed with these sources for the detection of optically thin and often short-lived species. Recent activities center on regenerative amplification of high-resolution solid-state lasers, development of tunable high-power, mid-IR lasers and short-pulse UV/VUV tunable lasers, and construction of an oil-free, rotating source beam machine for spectroscopy and dynamics studies of free radicals. In conjunction with these developments, this program provides for scientific and technical management of the construction of a chemical dynamics beamline at the Advanced Light Source.

\section{Efficient Generation of Tunable Narrowband Mid- Infrared Radiation in $\mathrm{KTiOAsO}_{4}$}

\section{A.H. Kung}

Potassium titanyl arsenate (KTA), an isomorph of potassium titanyl phosphate (KTP), has infrared transmission that extends to beyond $5.0 \mathrm{~mm}$. This suggests that the crystal has great potential for producing high-power tunable radiation in the critical mid-IR region of 3-5 mm. We report efficient generation of tunable narrowband midIR radiation in KTA by difference frequency mixing of an injection-seeded pulsed Ti:sapphire ring laser and a $Q$ switched Nd:YAG laser. High-power output from 2.6$5.3 \mathrm{~mm}$ is obtained.

*This work was supported by the Director, Office of Energy Research, Office of Basic Energy Sciences, Chemical Sciences Division, of the U.S. Department of Energy under Contract No. DE-AC03-76SF00098.
The experiment utilizes a regeneratively amplified KTP optical parametric oscillator (OPO-regen). This device produces single-frequency tunable 2 -nsec pulses at $\sim 100 \mathrm{~mJ}$. Residual $1.064 \mathrm{~mm}$ radiation from the Nd:YAG laser used to pump the power amplifier is combined with the OPO-regen pulses in a KTA crystal. Two KTA crystals are used. With a hydrothermal-grown KTA crystal that is cut at $\mathrm{q}=31^{\circ}, \mathrm{f}=90^{\circ}$ ( $\mathrm{y}-\mathrm{z}$ plane) and by tuning the OPOregen from $800 \mathrm{~nm}$ to $890 \mathrm{~nm}$, IR output from $3.0 \mathrm{~mm}$ to $5.3 \mathrm{~mm}$ is obtained. The long wavelength cutoff in IR generation is limited by absorption in the crystal. Output linewidth is estimated to be $0.5 \mathrm{~cm}^{-1}$, limited by that of the unseeded Nd:YAG laser. Narrowband continuous scanning and stable operation in the IR is demonstrated by obtaining an absorption spectrum of a $80-\mathrm{cm}$ path of atmospheric $\mathrm{CO}_{2}$.

For optimal conversion efficiency a flux-grown KTA crystal cut at $q=40^{\circ}, \mathrm{f}=0^{\circ}$ ( $\mathrm{x}-\mathrm{z}$ plane) is used. The effective nonlinearity for phase-matching in the $x-z$ plane is approximately 2.45 times higher than in the $y-z$ plane, making this orientation more desirable for high-efficiency generation. At an input energy of $115 \mathrm{~mJ}$ at $760 \mathrm{~nm}, 16 \mathrm{~mJ}$ of IR at $2.66 \mathrm{~mm}$ is measured. This corresponds to a peak power of $8 \mathrm{MW}$ and a photon conversion from the tunable input to IR output of $54 \%$. At $4.5 \mathrm{~mm}$, the measured photon conversion is $26 \%$, providing over $2.5 \mathrm{MW}$ of peak IR power. At this wavelength, pump depletion is $34 \%$, indicating the output efficiency is limited by bulk absorption in the KTA crystal.

These results show that KTA is an excellent crystal for generating high power mid-IR in the critical $3-5 \mathrm{~mm}$ region.

\section{Mid-Infrared Generation Using Dye Lasers in $\mathrm{KTiOAsO}_{4}$ vs. $\mathrm{LiIO}_{3}$}

\section{A. H. Kung, S. Fei, and H. L. Strauss (Publication 3)}

Infrared pulses have been generated in the 3.6 to $5.4 \mathrm{~mm}$ range by difference frequency mixing in KTA and $\mathrm{LiIO}_{3}$. A $532 \mathrm{~nm}$ pulse was mixed with a dye laser pulse in the crystals. The infrared power over the infrared range is comparable for the two crystals, but KTA has a higher damage threshold and less absorption.

\section{High Resolution High Power Tunable Infrared Laser}

\section{A.H. Kung}

We have successfully used high order Raman shifting in $\mathrm{H}_{2}$ (RSH) of single frequency and near diffraction 
limited Ti:sapphire laser system in hydrogen to generate IR with 11-4 mJ of IR light at 3-4 $\mu \mathrm{m}$. We have also used differential frequency generation (DFG) in the new KTA crystal with YAG $(1.064 \mu \mathrm{m})$ to produce $12-5 \mathrm{~mJ}$ at 3$5 \mu \mathrm{m}$. In completion of this laser system, we will use the scheme combining the DFG and the first order RSH to cover the whole middle infrared region. We will first differentiate Ti:sapphire (725-825 nm) with YAG second harmonic $(532 \mathrm{~nm})$ to produce $1.3-2.0 \mu \mathrm{m}$, and then differentiate this near IR light with YAG $(1.054 \mu \mathrm{m})$ to produce $60-30 \mathrm{~mJ}$ of near IR $(1.3-2.0 \mu \mathrm{m})$ and $20-10 \mathrm{~mJ}$ of first part middle $\mathbb{R}(2.0-4.0)$. Finally, we will use first order RSH in the multiple pass cell to convert of the near IR $(1.3-2.0 \mu \mathrm{m})$ light into second part middle IR $(4-10 \mu \mathrm{m})$ with the power of $10-2 \mathrm{~mJ}$.

\section{Work in Progress}

\section{IR-VUV Photoionization Spectroscopy}

\section{A.H. Kung and A.G. Suits}

We are exploring a sensitive IR spectroscopy method combining IR excitation with VUV photoionization detection. We set the VUV wavelength below the ionization potential from the ground vibrational state but enough to ionize vibrationally excited molecules. We scan the IR laser and monitor the mass-selected ion signal in a molecular beam machine. We are first trying out this method with $\mathrm{NH}_{3}$ where we use $3.4 \mu \mathrm{m}$ to excite second overtone of the umbrella bending vibration and use $125 \mathrm{~nm}$ VUV to do the ionization. The weak overtone transition will test the detection limit of this method. We will extend such studies to the free radicals, such as $\mathrm{C}_{3} \mathrm{H}_{7}$. In gas phase, this has not been studied partly owing to difficulty in producing high-density pure free radicals and partly owing to low sensitivities of conventional IR spectroscopy. We produce the free radicals in the molecular beam by either pyrolysis or photolysis of certain precursor molecules. The low I.P.s, $8.1 \mathrm{eV}$ for $n-\mathrm{C}_{3} \mathrm{H}_{7}$ and $7.5 \mathrm{eV}$ iso- $\mathrm{C}_{3} \mathrm{H}_{7}$, require quite low-energy VUV photons. As a result, there will be no any other stable molecules to be ionized in the beam. The IR spectroscopy of $\mathrm{C}_{3} \mathrm{H}_{7}$ will open the door for $\mathrm{IR}$ pump photodissociation dynamics for further studies.

\section{Construction of Rotating Source Photodissociation Apparatus}

\section{A.H. Kung, A.G. Suits, and Y.T. Lee}

This rotating molecular beam source machine for studying photodissociation is similar to another highly productive machine in the group, but is totally free of oil since it employs all magnetic bearing turbo molecular pumps. It will allow us to study hydrocarbon radicals that have in the past been prohibitive owing to high background. We are currently testing and completing this apparatus. We will use this machine with the tunable IR laser source to study the photodissociation of free radicals, such as $\mathrm{C}_{3} \mathrm{H}_{7}$,

$$
n-\mathrm{C}_{3} \mathrm{H}_{7} \rightarrow \mathrm{C}_{3} \mathrm{H}_{6}+\mathrm{H} \quad, \quad \Delta \mathrm{H}=37 \mathrm{kcal} / \mathrm{mol}
$$

and

$$
n-\mathrm{C}_{3} \mathrm{H}_{7} \rightarrow \mathrm{C}_{2} \mathrm{H}_{4}+\mathrm{CH}_{3} \quad, \quad \Delta \mathrm{H}=31 \mathrm{kcal} / \mathrm{mol}
$$

by selectively pumping the $\mathrm{CH}$ stretch and $\mathrm{C}-\mathrm{C}$ stretch individually. Then we use a certain wavelength $U V$ laser to dissociate the vibrationally excited $n-\mathrm{C}_{3} \mathrm{H}_{7}$. The FranckCondon overlap and UV photon energy will put the molecule onto a certain part of the repulsive potential surface that may selectively lead one of dissociation channels according to the initial vibrational selection. We will also study the multiple photon dissociation of radicals.

\section{FY 1994 PUBLICATIONS AND REPORTS}

\section{Refereed Journals}

1. A.H. Kung, "Narrowband Mid-Infrared Generation Using KTiOAsO 4 ," Appl. Phys. Lett. 65(9), 1082 (1994).

\section{Other Publications}

2. A.H. Kung, "Efficient Conversion of High Power Narrowband Ti:Sapphire Laser Radiation to the Mid-IR in KTA," Opt. Lett. (submitted).

3. A.H. Kung, S. Fei, and H.L. Strauss, "Mid-IR Generation Using Dye Lasers in KTA vs. $\mathrm{LilO}_{3}$," Appl. Phys. Lett. (submitted).

4. S. Fei, H.L. Strauss, and A.H. Kung, "Mid-IR Generation Using KTA," Bull. Am. Phys. Soc. (in press).

5. A.H. Kung, "Narrowband Mid-Infrared Generation Using $\mathrm{KTiOAsO}_{4}$," in Proceedings of the SPIE Conference on Solid State Lasers and Nonlinear Crystals, San Jose, CA., Feb. 1995.

6. A.H. Kung, "Experimental Aspects of Tunable Narrowband Mid-Infrared Generation in KTA," in Proceedings of the Advanced Solid State Lasers Technical Meeting, Memphis TN, Jan. 30-Feb 2, 1995.

7. A.H. Kung, "Efficient Generation of Tunable Narrowband Mid-IR Radiation in KTA," in Advanced Solid State Lasers, OSA Technical Digest (Optical Society of America, Washington D.C., 1995), p. 334. 


\section{Crossed Molecular Beams*}

\author{
Yuan T. Lee and Arthur Suits, Investigators
}

\section{INTRODUCTION}

The major thrust of this research project is to elucidate detailed dynamics of simple elementary reactions that are theoretically important, and to unravel the mechanism of complex chemical reactions or photochemical processes that play important roles in many macroscopic processes. Molecular beams of reactants are used to study individual reactive encounters between molecules or to monitor photodissociation events in a collision-free environment. Most of the information is derived from measurement of the product fragment energy, angular and state distributions. Recent activities are centered on the mechanisms of elementary chemical reaction dynamics in divalent systems, nonadiabatic collision dynamics, the dependence of the chemical reactivity of electronically excited atoms on the alignment of excited orbitals, the primary photochemical processes of polyatomic molecules, intramolecular energy transfer of chemically activated and locally excited molecules, the energetics of free radicals that are important to combustion processes, the infraredabsorption spectra of carbonium ions, and bond-selective photodissociation through electronic excitation. Recent activities are centered on the primary photochemical processes of radicals, decay and collision dynamics of highly excited Rydberg states of atoms and molecules, and the energetics of hydrocarbon radicals; photodissociation dynamics and thermochemistry of nitrogen-containing aromatic molecules; and the infrared spectra and structure of carbonium and silanium ions.

1. Vibration-Rotation Spectroscopy of the Hydrated Hydronium Ions $\mathrm{H}_{5} \mathrm{O}_{2}{ }^{+}$and $\mathrm{H}_{9} \mathrm{O}_{4}{ }^{+}$

\section{L.I. Yeh, Y.T. Lee, and J.T. Hougen}

High-resolution vibration-rotation spectra in the $\mathrm{OH}$ antisymmetric stretching region near $3700 \mathrm{~cm}^{-1}$ are reported for $\mathrm{H}_{5} \mathrm{O}_{2}{ }^{+}$and $\mathrm{H}_{9} \mathrm{O}_{4}{ }^{+}$. The clusters are produced in a corona discharge ion source, cooled by supersonic expansion, mass-selected, and trapped in an RF octopole ion trap. Spectroscopic interrogation using a two-color laser

\footnotetext{
* This work was supported by the Director, Office of Energy Research, Office of Basic Energy Sciences, Chemical Sciences Division of the U.S. Department of Energy under Contract No. DE-AC03-76SF00098.
}

scheme leads to rovibrational excitation of the trapped ions followed by preferential multiphoton dissociation of the vibrationally excited ions and detection of the resultant fragment ions. Many more lines appear in the partially resolved vibration-rotation spectrum of $\mathrm{H}_{5} \mathrm{O}_{2}{ }^{+}$than can be explained if the molecule is rigid, and we have assumed that these additional lines arise from tunneling splittings caused by large-amplitude internal motions in this ion. Despite the low signal-to-noise ratio, all the observed spectral features can be grouped into roughly $12 R$ branches with a line spacing only $14 \%$ less than the $B+C$ value calculated from the $a b$ initio structure. Theoretically expected splitting patterns were calculated using a formalism developed earlier for tunneling motions in hydrazine, since $\mathrm{H}_{2} \mathrm{~N}-\mathrm{NH}_{2} \mathrm{H}_{2} \mathrm{O}-\mathrm{H}^{+}-\mathrm{OH}_{2}$ are grouptheoretically similar if the central proton of the ion is located symmetrically between the two water molecules. We tentatively conclude that the 12 branches represent the overlapping of six tunneling-split components for the inphase and six for the out-of-phase $\mathrm{OH}$ antisymmetric stretching vibrations expected in this region, but the low signal-to-noise ratio in the present measurements prevented unambiguous comparison of theory and experiment.

\section{The Internal and Translational Energy Dependence of Molecular Condensation Coefficients: SF $_{6}$ and $\mathrm{CCl}_{4}$}

\section{S.J. Sibener and Y.T. Lee}

In this paper we describe a series of beam-surface scattering experiments that examine the internal and translational energy dependence of the molecular condensation probabilities for $\mathrm{CCl}_{4}$ or $\mathrm{SF}_{6}$ colliding with their respective condensed phases. It has been conclusively shown that thermal excitation of a polyatomic molecule's rotational and vibrational degrees of freedom can inhibit its probability of sticking upon impact with a cryogenically cooled surface. This effect is most pronounced in the limit of low incident kinetic energy, and essentially vanishes at higher velocities. As part of these experiments, we have also obtained the angular and velocity distributions for reflect $\mathrm{SF}_{6}$ and $\mathrm{Kr}$ that have been used to examine the energy and momentum exchange of these gases with their respective condensed phases. Our findings suggest that heterogeneous laser isotope separation schemes based on precollision molecular excitation may warrant further investigation. 


\section{Determination of the Barrier Height to $\mathrm{CH}_{3} \mathrm{CO}$ Dissociation}

\section{S. North, D.A. Blank, and Y.T. Lee}

The photodissociation of acetyl chlori de at $248 \mathrm{~nm}$ has been investigated by the technique of photofragment translational spectroscopy. A comparison of the translational energy distributions required to fit the chlorine atom and the acetyl radical indicates that a significant fraction $(\approx 35 \%)$ of the $\mathrm{CH}_{3} \mathrm{CO}$ fragments undergo secondary decomposition to $\mathrm{CH}_{3}$ and $\mathrm{CO}$. From analysis of the center-of-mass translational energy distributions, a value of $17 \pm 1 \mathrm{kcal} / \mathrm{mol}$ for the barrier height to acetyl radical dissociation has been determined.

\section{The Ultraviolet Photodissociation Dynamics of Pyrrole}

\section{D.A. Blank, S. North, and Y.T. Lee}

Photofragment translational spectroscopy was used to study the photodissociation of pyrrole at 193 and $248 \mathrm{~nm}$ under collision-free conditions. Five primary dissociation channels were observed at $193 \mathrm{~nm}$. Two channels resulted from cleavage of the $\mathrm{N}-\mathrm{H}$ bond to yield $\mathrm{H}+$ pyrrolyl radical with one channel following internal conversion (IC) to the ground state $(\approx 21 \%)$ and the other originating from electronically excited pyrrole $(\approx 30 \%)$. Two dissociation channels involved elimination of HCN following IC. One channel producing $\mathrm{HCN}+$ vinylmethylene $(\approx 25 \%)$ following ring opening and hydrogen migration and the other proceeding via a bridged $3 \mathrm{H}$-pyrrole intermediate to form $\mathrm{HCN}+$ cyclopropene $(\approx 24 \%)$. The last channel at $193 \mathrm{~nm}$ involved IC to the ground state followed by ring opening and $\mathrm{N}-\mathrm{C}$ bond cleavage to form $\mathrm{NH}+$ $\mathrm{CHCCHCH}_{2}(<1 \%)$. At $248 \mathrm{~nm}$ three dissociation channels were observed, all of which involved the elimination of atomic hydrogen. Analogous to the results at $193 \mathrm{~nm}$, two of these channels resulted from cleavage of the $\mathrm{N}-\mathrm{H}$ bond with one channel following IC $(\approx 42 \%)$ and the other dissociating from an excited electronic state $(\approx 47 \%)$. The third dissociation channel at $248 \mathrm{~nm}$ involved the cleavage of one of the two C-H bonds in electronically excited pyrrole $(\approx 11 \%)$. Translational energy distributions were determined for all observed dissociation channels. From consideration of the maximum translational energy of the photofragments $D_{0}(\mathrm{C}-\mathrm{H})=112.5 \pm 1 \mathrm{kcal} / \mathrm{mol}$ and $\Delta H_{\mathrm{f}}$ (pyrrolyl radical $)=62 \pm 2 \mathrm{kcal} / \mathrm{mol}$ were determined.

\section{The Photodissociation of Pyridine at $193 \mathrm{~nm}$}

\section{K.A. Prather and Y.T. Lee}

Using photofragmentation translational spectroscopy, the primary processes involved in the photodissociation of pyridine are investigated at $193 \mathrm{~nm}$. Upon absorption of a single photon, $\mathrm{HCN}+\mathrm{C}_{4} \mathrm{H}_{4}, \mathrm{CH}_{3}+\mathrm{C}_{4} \mathrm{H}_{2} \mathrm{~N}, \mathrm{C}_{3} \mathrm{H}_{3}+$ $\mathrm{C}_{2} \mathrm{H}_{2} \mathrm{~N}$, and $\mathrm{H}+\mathrm{C}_{5} \mathrm{H}_{4} \mathrm{~N}$ are the observed primary pathways, with relative yields of $59 \%, 21 \%, 11 \%$, and $9 \%$, respectively. This paper reports the experimental details and results of this study. Comparison of the results of this study with a previous study in our laboratory investigating the photolysis of benzene serves as a direct measure of the strong influence substitution of a heteroatom in an aromatic ring can have on a molecule's photochemistry.

\section{Photodissociation of the Methyl Radical 3s Rydberg State}

\section{S. North, D.A. Blank, P. Chu, and Y.T. Lee}

The photodissociation dynamics of methyl radical have been investigated at $193.3 \mathrm{~nm}$ using photofragment translational spectroscopy. The formation of $\mathrm{CH}_{2}$ and $\mathrm{H}\left({ }^{2} \mathrm{~S}\right)$ was the only dissociation pathway observed. Although it is not possible to assign the spin state of the methylene unambiguously, we believe that methylene is produced predominately in the ${ }^{1} \mathrm{~A}_{1}$ excited state. The translational energy distribution of the products is peaked at $\sim 13 \mathrm{kcal} / \mathrm{mol}$ that is consistent with the magnitude of the exit barrier on the excited state potential energy surface. The breadth of the distribution suggests that the methyl radicals dissociate from a wide range of geometries. From the photofragment angular distribution an anisotropy parameter of $\beta=-0.9 \pm 0.1$ was determined.

\section{Evidence for Stepwise Dissociation Dynamics in} Acetone at $248 \mathrm{~nm}$ and $193 \mathrm{~nm}$

\section{S.W. North, D.A. Blank, J.D. Gezelter, C.A. Longfellow, and Y.T. Lee}

The technique of molecular beam photofragment translational spectroscopy has been used to study the dissociation of acetone following $S_{1} \leftarrow S_{0}(248 \mathrm{~nm})$ and $S_{2} \leftarrow$ $S_{0}(193 \mathrm{~nm})$ excitation. Excitation at $248 \mathrm{~nm}$ resulted in the production of $\mathrm{CH}_{3}$ and $\mathrm{CH}_{3} \mathrm{CO}$ with $14.2 \pm 1.0 \mathrm{kcal} /$ 
mol on average of the available energy appearing as translation of the photofragments. Comparison of the measured $\left\langle E_{T}\right\rangle$ with values reported at $266 \mathrm{~nm}$ suggest that the energy partitioning is dominated by the exit barrier caused by an avoided crossing on the potential energy surface. A substantial fraction $(30 \pm 4 \%$ ) of the nascent acetyl radicals from the primary dissociation contain sufficient energy to undergo spontaneous secondary decomposition. From the onset of the truncation of the $\mathrm{CH}_{3} \mathrm{CO} P\left(E_{\mathrm{T}}\right)$, a threshold of $17.8 \pm 3.0 \mathrm{kcal} / \mathrm{mol}$ for the dissociation of the acetyl radical has been determined in agreement with recent results on the photodissociation of acetyl chloride. The translational energy release in the dissociation of $\mathrm{CH}_{3} \mathrm{CO}$ closely matches the experimentally determined exit barrier. At 193 $\mathrm{nm}$ the only observed dissociation pathway was the formation of two methyl radicals and carbon monoxide. On average $-38 \%$ of the available energy is found in product translation suggesting that significant internal energy resides in the nascent $\mathrm{CH}_{3}$ fragments, consistent with the results of Hall et al. ${ }^{1}$ We conclude that the dynamics and energy partitioning for dissociation at $193 \mathrm{~nm}$ is similar to that at $248 \mathrm{~nm}$.

1. G.E. Hall, D. Vanden Bout, and T.J. Sears, "Photodissociation of Acetone at $193 \mathrm{~nm}$ : Rotational- and Vibrational-State Distributions of Methyl Fragments by Diode Laser Absorption/Gain Spectroscopy," J. Chem. Phys. 94, 4182 (1991).

\section{Enhancements in the Lifetimes of NO Rydberg States in DC Electric Fields: Implications for ZEKE- Experiments}

\section{M.J.J. Vrakking and Y.T. Lee}

Lifetimes of NO p- and f-orbital Rydberg states ( $\mathrm{n}=$ 40-122) have been measured under near field-free conditions as well as in the presence of small DC electric fields. The field causes an increase in the observed lifetimes, which is attributed to Stark-mixing of the predissociative $\mathrm{p}$ - and f-orbitals with high- $l$ orbital angular momentum states. The results indicate that under the conditions existing in high-resolution Zero-ElectronKinetic-Energy PhotoElectron Spectroscopy (ZEKE-PES) experiments, the ZEKE Rydberg states are $l$-mixed.

\section{Work in Progress}

Considerable effort is currently directed to an investigation of the dissociation dynamics of hydrocarbon radicals. A pulsed pyrolytic source has been developed for generating intense beams of cooled radicals. The first investigation into the photochemistry of allyl radical suggest the formation of methyl + acetylene as a minor channel in addition to the dominant hydrogen atom elimination. Studies of the photochemistry of methyl and ethyl radical promise further insight into the thermochemistry and dissociation dynamics of these species. Pyrolytic fragmentation is of central importance in combustion processes, and a study currently under way using IR multiphoton excitation of fluorocarbons promises to yield valuable insight into the ground state dissociation processes of these molecules.

A very well-defined beam of $O\left({ }^{1} D\right)$ has recently been developed using photolysis of ozone in the expansion from a high-pressure pulsed nozzle. The result is a pulsed beam characterized by a very narrow velocity distribution that will yield high-resolution $O\left({ }^{1} D\right)$ reactive scattering experiments. Initial studies focus on an understanding of the dynamics of $\mathrm{O}\left({ }^{1} \mathrm{D}\right)$ reactions with $\mathrm{H}_{2}$ and hydrocarbons.

Recent studies in the IR spectroscopy of solvated carbonium ions have been quite fruitful. "Consequence spectroscopy" enables us to record spectra that are massspecific in both parent and daughter ions. Using this technique with isotope labeling, we have shown that proton exchange is rapid (barrier $<9 \mathrm{kcal} / \mathrm{mole}$ ) between carbons in $\mathrm{CH}_{5}{ }^{+}\left(\mathrm{CH}_{4}\right)$. Further studies of solvation series of carbonium ions, as well as studies of the spectroscopy of radical cations such as $\mathrm{CH}_{3} \mathrm{OH}^{+}, \mathrm{C}_{2} \mathrm{H}_{5} \mathrm{OH}^{+}$and $\mathrm{CH}_{3} \mathrm{COCH}_{3}{ }^{+}$, are currently under way.

We have recently succeeded in producing an intense supersonic lithium atomic beam and exciting it to the $2 \mathrm{P}$ state. Studies of the reaction dynamics of these excited atoms and the orbital alignment dependence of the product distributions promise detailed insight into systems that may be directly and meaningfully compared to accurate quantum mechanical calculations.

Considerable work has been done on the photochemistry of jet-cooled radicals, and studies continue along these lines. An ultrahigh resolution $\mathrm{H}$ atom translational spectrometer has been developed, modeled on the technique of Welge and coworkers. This method is being successfully applied to a study of the photodissociation of methyl radicals at $212 \mathrm{~nm}$.

Pyrazine has been used in recent studies of intermolecular energy transfer. We have recently begun an extensive investigation of the primary photochemistry of pyrazine in the ultraviolet. We have identified several product channels that follow the absorption of a single UV photon, including impulsive release of $\mathrm{HCN}$ with substantial translational energy.

Reactive scattering of sodium dimers is currently under investigation, extending our recent investigation of the chemistry of divalent systems. Reaction with methanol indicates the formation of its sodium adduct, in contrast to the reaction with barium that was observed to form the methoxide. Reaction of sodium dimer with oxygen and ozone are currently under study. Other reactive scattering 
studies currently in development include reactive scattering of hydroxyl and hydrocarbon radicals, development of a pyrolytic $O$ atom source, and development of a laser vaporization carbon atom source for reactive scattering with unsaturated hydrocarbons.

We continue to extend our study of the IR spectroscopy of carbonium and silanium ions, with comparison to molecular dynamics simulations of these systems. The results confirm the extremely floppy nature of $\mathrm{CH}_{5}{ }^{+}$, and show enhanced stablility with the successive addition of solvent atoms and molecules. The simulations enable us to see that solvent hydrogen molecules, for example, preferentially bind to $\mathrm{H}$ atoms involved in the 3center, 2-electron bond.

An exciting new devlopment in the coming year is the commissioning of the Chemical Dynamics Beamline at the Advanced Light Source. This will include a rotating source crossed-beam machine using the intense undulator radiation for soft, nearly background-free ionization. Primary photochemistry of radicals and reactive scattering of hydrocarbons, with direct identification of reaction products, will be possible.

\section{FY 1994 PUBLICATIONS AND REPORTS}

\section{Refereed Journals}

1. L.I. Yeh, Y.T. Lee, and J.T. Hougen, "Vibration-Rotation Spectroscopy of the Hydrated Hydronium Ions $\mathrm{H}_{5} \mathrm{O}_{2}{ }^{+}$and $\mathrm{H}_{9} \mathrm{O}_{4}{ }^{+}, "$ J. Mol. Spec. 164, 473 (1994); LBL-32591.

2 S.J. Sibener and Y.T. Lee, "The Internal and Translational Energy Dependence of Molecular Condensation Coefficients: $\mathrm{SF}_{6}$ and $\mathrm{CCl}_{4}$," J. Chem. Phys. 101, 1693 (1994); LBL-34775.

3. S. North, D.A. Blank, and Y.T. Lee, "Determination of the Barrier Height to $\mathrm{CH}_{3}$ CO Dissociation," Chem. Phys. Letts. 224, 38 (1994); LBL-35383.

4. D.A. Blank, S. North, and Y.T. Lee, "The Ultraviolet Photodissociation Dynamics of Pyrrole," Special Issue, Chem. Phys. 187, 35 (1994); LBL-35874.

5. K.A. Prather and Y.T. Lee, "The Photodissociation of Pyridine at 193 nm," Israel J. Chem. 34, 43 (1994); LBL36776.

\section{LBL Reports}

6. S. North, D.A. Blank, P. Chu, and Y.T. Lee, "Photodissociation of the Methyl Radical 3s Rydberg State," J. Chem. Phys. (in press); LBL-35927.

7. S. W. North, D.A. Blank, J.D. Gezelter, C.A. Longfellow, and Y.T. Lee, "Evidence for Stepwise Dissociation Dynamics in Acetone at $248 \mathrm{~nm}$ and $193 \mathrm{~nm}$," J. Chem. Phys. (in press); LBL-36417.

8. M.J.J. Vrakking and Y.T. Lee, "Enhancements in the Lifetimes of NO Rydberg States in DC Electric Fields: Implications for ZEKE-Experiments," Phys. Rev. A (in press); LBL-35627.

\section{Invited Talks}

9. Y.T. Lee, "Elementary Reactions Involved in the Diamond Film Synthesis," International Conference on Diamond Film Synthesis, National Tsinghua University, Hsinchu, Taiwan, March 25, 1994.

10. Y.T. Lee, "Laser Induced Chemical Reactions," Li Kah Sheng Lecture, Hong Kong University, Hong Kong, April 11, 1994.

11. Y.T. Lee, "Molecular Beam Studies of Dynamics of Molecular Dissociation," Chemistry Department, Hong Kong University, Hong Kong, April 12, 1994.

12. Y.T. Lee, "Following in the Footsteps of James Franck," Israel Academy of Sciences, Jerusalem, Israel, August 25, 1994.

13. Y.T. Lee, "Intermediates of Proton Transfer Reactions," International Molecular Beam Symposium, Salamanca, Spain, September 2, 1994.

14. Y.T. Lee, "Promotion of Chemical Reactions by Laser Excitation," Academia Sinica, Taipei, Taiwan, September $10,1994$.

15. Y.T. Lee, "Combustion and Air Pollution," 75th anniversary of IUPAC, Taipei, Taiwan, September 12, 1994.

16. Y.T. Lee, "Combustion and Atmospheric Chemistry," IUAPPA Conference, Academia Sinica, Taipei, Taiwan, November 2, 1994.

17. Y.T. Lee, "Steering Chemical Reactions Through Laser Excitation," Asian Academy of Sciences, Bangalore, India, December 19, 1994.

18. Y.T. Lee, "Concerted Decomposition of Cyclic and Heterocyclic Compounds," Linus Pauling Lecture, Indian Institute of Science, Bangalore, India, December 21, 1994. 


\section{Molecular Interactions*}

William A. Lester, Jr., Investigator

\section{INTRODUCTION}

This research program is directed at extending fundamental knowledge of atoms and molecules including their electronic structure, mutual interaction, collision dynamics, and interaction with radiation. The approach combines the use of $a b$ initio basis set methods and the recently developed quantum Monte Carlo (QMC) to describe electronic structure, intermolecular interactions, and other properties. Further, the approach uses various methods for characterizing inelastic and reactive collision processes. Present activity is focused primarily on the development and application of the QMC method.

1. Molecular States of the CO Interaction with the $3 d$ Metal Surfaces (Publication 1)

\section{A.C. Paväo, ${ }^{\dagger}$ M.M. Soto ${ }^{\ddagger}$ W. A. Lester, Jr., S.K. Lie, ${ }^{\S}$ B.L. Hammond, "and C.A. Taft"}

$A b$ initio molecular orbital calculations of the carbonyls of $3 d$-transition metals are used to indicate the tilted metal-CO complex as the molecular state precursor of $\mathrm{CO}$ dissociation on $3 d$ surfaces. The present resonatingvalence-bond mechanism for $\mathrm{CO}$ dissociation suggests that the tilted state may be observed in all dissociative $3 d$ surfaces, that is, from Sc to Fe. Calculations of scandium monocarbonyl are used to understand the effects of inclination on electronic configuration, and calculations of $\mathrm{Cr}_{2} \mathrm{CO}$ cluster are used to study $\mathrm{CO}$ dissociation on the $\mathrm{Cr}(110)$ surface and to explain the substantial reduction of $\mathrm{CO}$ binding modes found for the tilted state.

\footnotetext{
†Permanent address: Departamento de Química Fundamental, Universidade Federal de Pernambuco, Pernambuco, Brazil.

$¥$ Present address: MDL Information Systems, Inc., San Leandro, CA.

\$Permanent address: Departamento de Fisica, Universidade Federal Fluminense, 24249 Nieteroi, Rio de Janeiro, Brazil.

"Permanent address: Fujitsu America, Inc., San Jose.

Ipermanent address: Centro Brasileiro de Pesquisas Físicas, Rio de Janeiro, Brazil.
}

2. Characteristics of Electron Movement in Variational Monte Carlo Simulations (Publication 2)

Z. Sun ${ }^{\dagger}$ M.M. Soto, $\ddagger$ and W.A. Lester, Jr.

Improving the efficiency of quantum Monte Carlo (QMC) to make possible the study of large molecules poses a great challenge. Evaluating the efficiency of Monte Carlo sampling, however, is at a rudimentary level and in need of new algorithms. Instead of the autocorrelation time as an efficiency measure for Monte Carlo simulations, we propose a direct method to characterize the movement of electrons in atoms or molecules during variational Monte Carlo (VMC) computations. Further, the approach makes possible an efficient diagnostic tool to understand objectively many interesting issues in QMC. The usefulness of the method is demonstrated by comparisons among improved Metropolis algorithms and the original Metropolis algorithm. We also present an optimization method for choosing step sizes for Monte Carlo walkers. These step sizes are governed by the acceptance ratio of the electrons closest to the heaviest nucleus. Step sizes obtained for $\mathrm{Ne}$ and $\mathrm{Ar}$ are consistent with those obtained by the autocorrelation approach. Our study shows no evidence to support distinctions of core and valence electrons during simulations, and confirms that, in most cases, moving electrons individually is more efficient than moving all the electrons at once. We find that "trapped" or "stale" configurations are due to a large quantum force. From this study we can infer that "trapping" occurs at points where the wave function is close to zero. We present a cutoff as a solution to this problem for guided VMC.

\footnotetext{
†Permanent address: Institute of Mechanics, Chinese Academy of Sciences, Beijing, People's Republic of China.

$\ddagger$ Present address: MDL Information Systems, Inc., San Leandro, CA.
}

3. Hyperconjugation and Charge Distribution in Alicyclic Alcohols and Exo- and Endo-norbornol (Publication 3)

J.G. R. Tostes, ${ }^{\dagger} J$. W. de M. Carneiro, ${ }^{\dagger}$ S. K. Lie, ${ }^{\dagger}$

P.R. Seidl, ${ }^{\ddagger}$ C.A. Taft, ${ }^{\S}$ M.M. Soto," W.A. Lester, Jr., and B.L. Hammond ${ }^{\text {II }}$

$A b$ initio and $\mathrm{MM} 2$ calculations are used to investigate charge distributions in selected alcohols (methanol, ethanol, 2-propanol and $t$-butanol and exo- and endo-norbornol). The calculated net atomic charges obtained using full optimized geometries with a range of basis sets (STO-3G, $\left.4-31 \mathrm{G}, 6-31 \mathrm{G}^{* *}\right)$ indicate that selected carbon and hydrogen atoms in the proximity of a nonbonding oxygen lone pair are more negatively charged and the carbon-
*This work was supported by the Director, Office of Energy Research, Office of Basic Energy Sciences, Chemical Sciences Division, of the U.S. Department of Energy under Contract No. DE-AC03-76SF00098. 
hydrogen bonds are longer than usual. Analysis of fully optimized geometries, HOMO populations, and net atomic charges for these alcohols suggests that the differences in charge distribution on the carbon and hydrogen atoms and their bond lengths can be explained in terms of hyperconjugation. This study shows that hyperconjugation can be a powerful tool for analyzing the effects of oxygen lone pairs on vicinal carbon and hydrogen atoms participating in $\mathrm{C}-\mathrm{C}$ and $\mathrm{C}-\mathrm{H}$ bonds in the vicinity of a hydroxyl group. The charge distributions also suggest that other atoms in close proximity are also sensitive to the hydroxyl lone pair, but to a smaller extent.

\footnotetext{
Permanent address: Universidade Federal Fluminense, Niteroi, RJ, Brazil.

† Permanent address: Centro de Tecnologia Mineral (CETEM), Cidade Universitaria, Rio de Janeiro, Brazil.

\$Permanent address: Centro Brasileiro de Pesquisas Físicas, Rio de Janeiro, Brazil.

"Present address: MDL Information Systems, Inc., San Leandro, CA. qPermanent address: Fujitsu America, Inc., San Jose.
}

\section{An Approach for Improved Variational Quantum Monte Carlo (Publication 4)}

\section{Z. Sun ${ }^{\dagger}$ M. M. Soto $\stackrel{\ddagger}{\ddagger}$ R.N. Barnett, and W.A. Lester, Jr.}

Sampling of core electrons in Monte Carlo approaches to electronic structure is a major bottleneck to efficient studies of large molecules. To overcome this problem, we propose an improved Metropolis algorithm for variational Monte Carlo that includes the second derivatives (Hessian matrix) of the pseudopotential $P=-\ln \left|\Psi_{T}\right|^{2}$ in its transition probability in addition to the commonly used first derivatives (or quantum force). To minimize computational effort, we use only the diagonal elements of the Hessian matrix, which are readily obtained from information already available in the Monte Carlo computation. We analyze the effect of these diagonal terms on the transition probability and core-electron sampling. The approach automatically reduces the step sizes of the innermost electrons and does not require further considerations such as choice of coordinate system or assignment of electrons to specific shells. In addition, heteronuclear molecules pose no difficulty for the present algorithm. Application of the method to representative systems, $\mathrm{Ne}, \mathrm{Ar}$, and $\mathrm{KCl}$, has shown that it increases the acceptance ratio of the innermost core electrons by a factor of 5 over previous algorithms.

\footnotetext{
Permanent address: Institute of Mechanics, Chinese Academy of Sciences, Beijing, People's Republic of China.

†Present address: MDL Information Systems, Inc., San Leandro, CA.
}

\section{FY 1994 PUBLICATIONS AND REPORTS}

\section{Refereed Journals}

1. A.C. Paväo, M.M. Soto, W.A. Lester, Jr., S.K. Lie, B.L. Hammond, and C.A. Taft, "Molecular States of the CO Interaction with the $3 d$-Metal Surfaces," Phys. Rev. B. 50(3), 1868 (1994); LBL-34391.

2. Z. Sun, M.M. Soto, and W.A. Lester, Jr., "Characteristics of Electron Movement in Variational Monte Carlo Simulations," J. Chem Phys. 100(2), 1278 (1994); LBL34733 .

3. J.G.R. Tostes, J.W. de M. Carneiro, S.K. Lie, P.R. Seidl, C.A. Taft, M.M. Soto, W.A. Lester, Jr., and B.L. Hammond, "Hyperconjugation and Charge Distribution in Alicyclic Alcohols and Exo- and Endo-Norbornol," J. Mole. Struct. (Theochem.) 306, 101 (1994); LBL- 35147.

4. Z. Sun, M.M. Soto, R.N. Barnett, and W.A. Lester, Jr., "An Approach for Improved Variational Quantum Monte Carlo," J. Chem Phys. 101 (1), 394 (1994); LBL-35233.

\section{LBL Reports}

5. R.N. Barnett, E.M. Johnson, and W.A. Lester, Jr., "Quantum Monte Carlo Determination of the Lithium $2^{2} \mathrm{~S} \rightarrow 2^{2} P$ Oscillator Strength: Higher Precision," Phys. Rev. A. (submitted July 1994); LBL-36488.

6. J.A. Odutola and W.A. Lester Jr., "Molecular Reorientation Cross Sections in Collisions of $\mathrm{He}\left({ }^{1} \mathrm{~S}\right)+\mathrm{D}_{2}\left(\mathrm{~B}^{1} \Sigma_{\mathrm{u}}^{+}\right)$, HD $\left(B^{1} \Sigma_{\mu}^{+}\right)^{*}$," J. Chem. Phy.s, (submitted August 1994); LBL36010 .

7. A.C. Paväo, B.L. Hammond, M.M. Soto, W.A. Lester, Jr., and C.A. Taft, "Theoretical Study of the CO Interaction with the Cr(110) Surface," Surf. Sci. (submitted September 1994); LBL-36423.

8. A.C. Pavão, C.A. Taft, T.C. Guimãraes, and W.A. Lester, Jr., "Breaking Bonds of Diatomic Molecules on Transition Metal Surfaces," Trends in Chem. Phys. (in press); LBL36648 .

\section{Invited Talks}

9. W.A. Lester, Jr., invited appearance at the PBS special aired nationally, entitled "Your Toxic Trash," October 1993.

10. W.A. Lester, Jr., "Expanding the Pool: Further Thoughts," Lawrence Berkeley Laboratory, Berkeley, CA, February, 1994.

11. W.A. Lester, Jr., "Computers: The Machines, Science, People, and Careers," Second Annual Computer and Computational Science Awareness Program for Minority Youth. The Center for Research on Parallel Computation, California Institute of Technology, Pasadena, CA, March 1994.

12. W.A. Lester, Jr., "Cultural Linkages in Science Education," The Oakland Museum of California, Oakland, CA, April 1994.

13. W.A. Lester, Jr., "Computational Chemistry: Current Status and Future Prospects," California Association of Chemistry Teachers Conference, Lawrence Berkeley Laboratory, Berkeley, CA, August 1994. 


\section{Theory of Atomic and Molecular Collision Processes*}

\author{
William H. Miller, Investigator
}

\section{INTRODUCTION}

This research is primarily involved with the development and application of theoretical methods and models for describing atomic and molecular collision processes and chemical reaction dynamics. Specific topics of interest have included the theory of inelastic and reactive scattering, collision processes involving electronically excited atoms or molecules, collisional ionization phenomena, statistical theories of chemical reactions, scattering of atoms and molecules from surfaces, and the interactions of molecular systems with high power laser radiation.

Research deals with both the development of rigorous theoretical approaches that are applicable to simple chemical systems, and also more approximate methods that are applicable to complex chemical systems, e.g., polyatomic molecules and their reactions. Even in the latter area, though, the goal is to develop approaches that can utilize $a b$ initio quantum chemical calculations of the potential energy surface (in the Born-Oppenheimer approximation) as direct input into the dynamical treatment, and thus, to as great an extent as possible, have a truly predictive theory.

\section{Semiclassical Correction for Quantum Mechanical Scattering (Publication 5)}

\section{S. Keshavamurthy and W.H. Miller}

A straightforward theoretical prescription is described for combining any approximate quantum scattering calculation with a semiclassical correction. The correction involves the standard semiclassical approximation to the time evolution operator, so that only real time trajectories are needed; by transforming to an initial value representation the calculations require only an average over the phase space of initial conditions. To the extent that the semiclassical approximation is accurate, the net result for the S-matrix is exact. Application to one-dimensional barrier transmission shows the semiclassical approximation

\footnotetext{
*This work was supported by the Director, Office of Energy Research, Office of Basic Energy Sciences, Chemical Sciences Division of the U.S. Department of Energy under Contract No. DE-AC03-76SF00098.
}

to do a very good job, for energies above, near to, or far below the barrier.

2. Quantum Mechanical Calculations of the Rate Constant for the $\mathrm{H}_{2}+\mathrm{OH} \rightarrow \mathrm{H}+\mathrm{H}_{2} \mathrm{O}$ Reaction: Full-Dimensional Results and Comparison to Reduced Dimensionality Models (Publication 6) ${ }^{\dagger}$

\section{U. Manthe, ${ }^{\ddagger}$ T. Seideman, ${ }^{\S}$ and W.H. Miller}

The cumulative reaction probability is calculated for the $\mathrm{H}_{2}+\mathrm{OH} \rightarrow \mathrm{H}+\mathrm{H}_{2} \mathrm{O}$ reaction in its full (six) dimensionality for total angular momentum $\mathrm{J}=0$. The calculation, which should give the (numerically) exact result for the assumed potential energy surface, yields the cumulative reaction probability directly, without having to solve the complete state-to-state reactive scattering problem. Higher angular momenta $(J>0)$ were taken into account approximately to obtain the thermal rate constant $k(T)$ over the range $300^{\circ}<\mathrm{T}<700^{\circ}$. The result deviates significantly from the experimental rate constant, suggesting that the potential energy surface needs to be improved. A systematic series of reduced dimensionality calculations is carried out in order to characterize the behavior and reliability of these more approximate treatments; a comparison of the full-dimensional results with previous reduced dimensionality calculations is also made.

\footnotetext{
This work is also supported in part by the National Science Foundation, Grant CHE89-20690.

${ }^{\ddagger}$ Fakultät für Physik, Universität Freiburg, D-79104 Freiburg, Germany.

$\S$ Staecie Institute for Molecular Sciences, National Research Council of Canada, Ottawa, Ontario K1A OR6, Canada.
}

3. Initial State-Selected Reaction Probabilities for $\mathrm{OH}+\mathrm{H}_{2} \rightarrow \mathrm{H}+\mathrm{H}_{2} \mathrm{O}$ and Photodetachment Intensities for $\mathrm{HOH}_{2}-$ (Publication 7)

\section{W.H. Thompson and W.H. Miller}

We have used a discrete variable representation (DVR) with absorbing boundary conditions (ABC) to calculate initial state-selected reaction probabilities and photodetachment intensities. We apply this method to the $\mathrm{OH}+\mathrm{H}_{2}$ reaction constrained to a plane with the $\mathrm{OH}$ bond frozen. The calculated reaction probabilities have all the qualitative features observed in full-dimensional calculations. We have similarly computed arrangementselected photodetachment intensities for one geometry of the $\mathrm{HOH}_{2}{ }^{-}$anion. The resulting spectrum has a dominant peak which will present a test of the neutral potential energy surface upon comparison with experimental results. 
4. On the Relation Between Unimolecular Reaction Rates and Overlapping Resonances (Publication 8)

\section{U. Peskin, H. Reisler, ${ }^{\dagger}$ and William H. Miller}

Unimolecular decay processes are studied in the regime of overlapping resonances with the goal of elucidating how unimolecular reaction rates depend on resonance widths (the imaginary part of the Siegert eigenvalues). As illustrated analytically for onedimensional models and numerically for a more general random matrix version of Feshbach's optical model, transition state theory (TST, RRKM) provides the correct average unimolecular decay rate whether the resonances are overlapping or not. For all studied cases, the explicit "universal" dependence of the TST average rate on the average resonance width (for a given or an energy interval) is that of a saturation curve: in the regime of nonoverlapping resonances (i.e., weak coupling) the standard relation "unimolecular decay rate $=$ resonance width $/ \hbar$ " holds, but as the resonance overlap increases (strong coupling) the rate saturates, becoming practically independent of the average resonance width in the strong overlapping limit. On the basis of these conclusions, a discussion of what has been or can be measured in experiments of unimolecular decay that relates to the average decay rate and to the resonance widths is given.

${ }^{\mp}$ Department of Chemistry, University of Southern California, Los Angeles, CA 90089.

\section{Reactive Scattering Theory for Molecular} Transitions in Time-Dependent Fields (Publication 13)

\section{U. Peskin and W.H. Miller}

A new approach is introduced for computing probabilities of molecular transitions in time-dependent fields. The method is based on the stationary $\left(t, t^{\prime}\right)$ representation of the Schrödinger equation and is shown to be equivalent to infinite order time-dependent perturbation theory. Bound-to-bound (i.e., photoexcitation) and boundto-continuum (i.e., photoreaction) transitions are regarded as reactive collisions with the "time coordinate" as the reaction coordinate in an extended Hilbert space. A numerical method based on imposing absorbing boundary conditions for the time-coordinate in a discrete variable representation framework is introduced. A single operation of the Green's operator provides all the state-specific transition probabilities as well as partial state-resolved (inclusive) reaction probabilities. Illustrative numerical applications are given for model systems.

\section{Final State-Selected Spectra in Unimolecular} Reactions: A Transition-State-Based Random Matrix Model for Overlapping Resonances (Publication 14)

\section{U. Peskin, W.H. Miller, and H. Reisler ${ }^{\dagger}$}

Final state-selected spectra in unimolecular decomposition are obtained by a random matrix version of Feshbach's optical model. The number of final states that are independently coupled to the molecular quasibound states is identified with the number of states at the dividing surface of transition state theory (TST). The coupling of the transition state to the molecular complex is modeled via a universal random matrix effective Hamiltonian that is characterized by its resonance eigenstates and provides the correct average unimolecular decay rate. The transition from non-overlapping resonances that are associated with isolated Lorentzian spectral peaks, to overlapping resonances, associated with more complex spectra, is characterized in terms of deviations from a $\chi^{2}$-like distribution of the resonance widths and the approach to a random phase-distribution of the resonance scattering amplitudes. The evolution of the system from a tight transition state to reaction products is treated explicitly as a scattering process where specific dynamics can be incorporated. Comparisons with recently measured final state-selected spectra and rotational distributions for the unimolecular reaction of $\mathrm{NO}_{2}$ show that the present model provides a useful new approach for understanding and interpreting experimental results which are dominated by overlapping resonances.

\footnotetext{
Department of Chemistry, University of Southern California, Los Angeles, CA 90089.
}

\section{On the "Direct" Calculation of Thermal Rate Constants (Publication 15)}

\section{W.H. Thompson and W.H. Miller}

We present a new approach for the direct (and correct) calculation of thermal rate constants $k(T)$ ("direct" meaning that one avoids having to solve the state-to-state reactive scattering problem, and "correct" meaning that the method contains no inherent approximations). The rate constant is obtained from the long time limit of the flux-position correlation function, $\mathrm{C}_{\mathrm{f}, \mathrm{s}}(\mathrm{t})$, whose calculation is made efficient by taking advantage of the low rank of the flux operator. Specifically, the trace required to obtain $\mathrm{C}_{\mathrm{f}, \mathrm{S}}(\mathrm{t})$ is evaluated by a Lanczos scheme that calculates only the nonzero eigenvalues. The propagation in complex time, $t_{c}=t-i \hbar \beta / 2$ is carried out using a Chebychev expansion. This method is seen to be both accurate and efficient by 
application to the Eckart barrier, the collinear $\mathrm{H}+\mathrm{H}_{2}$ reaction, and the three-dimensional $\mathrm{D}+\mathrm{H}_{2}(\mathrm{~J}=0)$ reaction.

\section{Work in Progress}

Effort is being focused on the very recent (and efficient) approach for computing the thermal rate constant $k(T)$ "directly," analogous to the approach developed earlier for computing the cumulative reaction probability. The goal is to be able to deal accurately with simple reactions, and also to develop approximations that can treat complex reactions to an acceptable level of accuracy.

\section{FY 1994 PUBLICATIONS AND REPORTS}

\section{Refereed Journals}

1. S.M. Auerbach and C. Leforestier, "A New Computational Algorithm for Green's Functions: Fourier Transform of the Newton Polynomial Expansion," Comp. Phys. Comm. 78, 55 (1993); LBL-34570.

2. C. Leforestier and W.H. Miller, "Quantum Mechanical Calculations of the Rate Constant for the Reaction $\mathrm{H}+$ $\mathrm{O}_{2} \rightarrow \mathrm{OH}+\mathrm{O}$," J. Chem. Phys. 100, 733 (1994); LBL34817.

3. P. Saalfrank and W.H. Miller, "Quantum Mechanical Rates for Gas-Surface Processes," Surf. Sci. 303, 206 (1994).

4. S.M. Auerbach and W.H. Miller, "Efficient Polynomial Expansion of the Scattering Green's Function: Application to the $\mathrm{D}+\mathrm{H}_{2}(\mathrm{~V}=1)$ Rate Constant," J. Chem. Phys. 100, 1103 (1994); LBL-34739.

5. S. Keshavamurthy and W.H. Miller, "Semiclassical Correction for Quantum Mechanical Scattering," Chem. Phys. Lett. 218, 189 (1994); LBL-34949.

6. U. Manthe, T. Seideman, and W.H. Miller, "Quantum Mechanical Calculations of the Rate Constant for the $\mathrm{H}_{2}+$ $\mathrm{OH} \rightarrow \mathrm{H}+\mathrm{H}_{2} \mathrm{O}$ Reaction: Full Dimensional Results and Comparison to Reduced Dimensionality Problems," J. Chem. Phys. 101, 4759 (1994); LBL-35825.

7. W.H. Thompson and W.H. Miller, "Initial State-Selected Reaction Probabilities for $\mathrm{OH}+\mathrm{H}_{2} \rightarrow \mathrm{H}+\mathrm{H}_{2} \mathrm{O}$ and Photodetachment Intensities for $\mathrm{HOH}_{2}-$, J. Chem. Phys. 101, 8620 (1994); LBL-35826.

8. U. Peskin, H. Reisler, and W.H. Miller, "On the Relation between Unimolecular Reaction Rates and Overlapping Resonances," J. Chem. Phys. 101, 9672 (1994); LBL35939.

\section{Other Publications}

9. W.H. Miller, "Recent Developments in the Quantum Mechanical Theory of Chemical Reaction Rates," in New Trends in Reaction Rate Theory, eds. P. Hänggi and P. Talkner (Kluwer Academic Publishers, 1994), pp. 225; LBL-35145.

\section{LBL Reports}

10. S.M. Auerbach, "Modern Integral Equation Techniques for Quantum Reactive Scattering Theory," Ph.D. Thesis; LBL34923.

11. R. Hernandez, "Application of Semiclassical Methods to Reaction Rate Theory," Ph.D. Thesis; LBL-34925.

12. L.F. Gaucher, "A Discrete Variable Representation for Electron-Hydrogen Atom Scattering," Ph.D. Thesis; LBL36080.

13. U. Peskin and W.H. Miller, "Reactive Scattering Theory for Molecular Transitions in Time-Dependent Fields," J. Chem. Phys. (in press); LBL-36053.

14. U. Peskin, W.H. Miller, and H. Reisler, "Final StateSelected Spectra in Unimolecular Reactions: A TransitionState-Based Random Matrix Model for Overlapping Resonances," J. Chem. Phys. (in press); LBL-36466.

15. W.H. Thompson and W.H. Miller, "On the 'Direct' Calculation of Thermal Rate Constants," J. Chem. Phys. (submitted); LBL-36609.

\section{Invited Talks}

16. W.H. Miller, "Beyond Transition State Theory, A Rigorous Quantum Theory for Chemical Reaction Rates," Nagoya Symposium on Chemical Reaction Theory, Nagoya, Japan, November 15-18, 1993.

17. W.H. Miller, "Chemical Reaction Dynamics-Where Are We and Where Are We Going?", Symposium on Future Challenges in Molecular Physics, Göttingen, Germany, November 25-27, 1993.

18. W.H. Miller, "Beyond Transition State Theory, A Rigorous Quantum Theory for Chemical Reaction Rates," Awards Symposium, American Chemical Society National Meeting, San Diego, CA, March 12-15, 1994.

19. W.H. Miller, "Semiclassical Corrections to Quantum Mechanical Scattering," Symposium on Quantum Reactive Scattering, American Physical Society National Meeting, Crystal City, VA, April 18-22, 1994.

20. W.H. Miller, "Direct Calculation of the Cumulative Reaction Probability," Workshop on Quantum Mechanical Treatment of Atom Exchange Processes in Molecular Collisions, Harvard-Smithsonian Center for Astrophysics, Cambridge, MA, June 30-July 2, 1994.

21. W.H. Miller, “A Random Matrix/Transition State Model for the Probability Distribution of State-Specific Unimolecular Decay Rates," International Discussion Meeting of the Deutsche Bunsengesellschaft für Physikalische Chemie, Molecular Spectroscopy and Molecular Dynamics: Theory and Experiment, Grainau, Bavarian Alps, Germany, August 28-September 1, 1994.

22. W.H. Miller, "Chemical Reaction Rate Theory-From Transition State Theory to Rigorous Quantum Approaches," NATO Advanced Study Institute on Frontiers of Chemical Dynamics, Antalya, Turkey, September 5-17, 1994. 


\section{Selective Photochemistry*}

\section{Bradley Moore, Investigator}

\section{INTRODUCTION}

The fundamental goals of this work are to elucidate the molecular dynamics of energy transfer and chemical reaction processes. Lasers are used to prepare molecules in selected excited states, and a variety of spectroscopic probes are used to follow energy transfers and chemical reactions with quantum-state resolution. Ideally all quantum numbers are resolved in the initial excitation and for each of the product states. By exploring the dependence of processes on quantum state and other experimentally controllable parameters, it is usually possible to establish a physical model for the process. Optimally, accurate benchmarks are established for the quantitative test of first principles theory.

Chemical reactions usually require excitations corresponding to ultraviolet photon energies and hence the initial state is usually an electronically excited one. The first step is then to understand the transfer of electronic (and perhaps vibrational and even rotational) energy to different electronic states and to vibrational, rotational, and translational degrees of freedom. When the ketene molecule is placed into its first excited electronic state, strong electronic-vibrational and spin-orbit couplings produce a statistical mixture of excited singlet, triplet, and ground-singlet states. The lower the electronic energy the greater the vibrational energy and the greater the corresponding statistical weight. The lowest threshold for dissociation to $\mathrm{CH}_{2}+\mathrm{CO}$ is on the triplet surface. The total energy and angular momentum can be finely controlled. The microcanonical reaction rate, $k(E, J)$, and reaction product attributes can be studied to reveal the dynamics of the reaction in the transition state region. In this same energy region unimolecular exchange of the carbon atoms occurs and thus excitation of ${ }^{13} \mathrm{CH}_{2} \mathrm{CO}$ can yield ${ }^{13} \mathrm{CO}$ product. This reaction permits the first study of reaction rate resonances due to vibrational resonances in the reaction coordinate degree of freedom. These studies are providing an increasingly sharp picture of molecular dynamics in the region of a unimolecular transition state.

Lasers are particularly useful in studying the highly reactive species important in combustion, atmospheric reactions, and homogenous catalysis. Flash photolysis with a laser provides a controlled source of these species for

*This work was supported by the Director, Office of Energy Research, Office of Basic Energy Sciences. Chemical Sciences Division of the U.S. Department of Energy under Contract No. DE-AC03-76SF00098. study in gases or liquids. Laser spectroscopy provides excellent wavelength and time resolution for observation of the spectrum and study of the reaction kinetics of reactive species. Studies here concentrate on infrared absorption spectroscopic studies of hydrocarbon radicals important in combustion and atmospheric chemistry and, in collaboration with Robert Bergman and Charles Harris, coordinatively unsaturated organometallics important in $\mathrm{CH}$ activation chemistry.

\section{Transition State Vibrational Level Thresholds for the Dissociation of Triplet Ketene (Publication 17)}

\section{S.K. Kim, E.R. Lovejoy, and C.B. Moore}

The stepwise increase of rate constants with increasing energy observed in ketene dissociation gives direct experimental evidence for the utility of the fundamental tenets of the RRKM theory that (1) the rate of reaction is controlled by flux through quantized transition-state thresholds, (2) the vibrational energy in the excited molecule is distributed statistically among all the vibrational degrees of freedom, and (3) the intramolecular vibrational redistribution (IVR) rate is much faster than the reaction rate. Further, and in contrast to the usual assumption in applying RRKM theory, $\mathrm{K}$ is seen to be a good quantum number for $\mathrm{J} \leq 6$. The experimental observation of the quantized transition-state thresholds as distinct steps in rate constants confirms the original concept of transition state theory that passage through the transition state is "vibrationally adiabatic" in the sense that energy is tied up in vibrational modes orthogonal to the reaction coordinate during the brief passage through the transition state region. The comparison of RRKM calculations to the experiment, Figure 1-1, also supports the basic tenet of transition state theory that systems cross the transition state directly from reactants and proceed to products without recrossing. The relative step heights are quite well reproduced by RRKM calculations when unity is used for the transmission coefficient for each quantized transition state. In principle, a value significantly less than unity is also possible in the unlikely circumstance that it is identical for every transition state vibrational level threshold.

However, straightforward application of RRKM theory, even with one-dimensional tunneling along the reaction coordinate, does not reproduce the detailed shape of each step. Several steps exhibit small peaks that are clearly inconsistent with the monotonically increasing rate predicted by RRKM theory. The threshold step for the zero-point vibrational level exhibits a less rapid rise than those for excited vibrational levels. The latter rise more rapidly than could be consistent with one-dimensional 


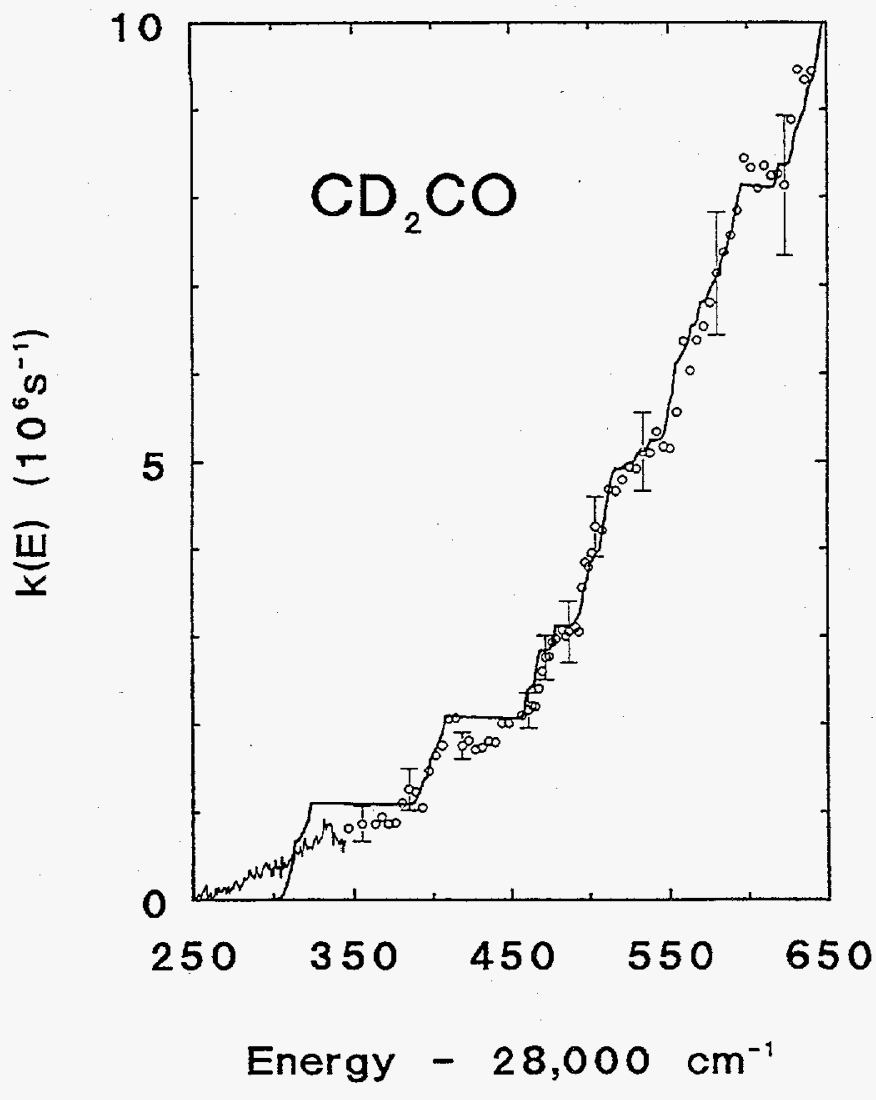

Figure 1-1. Rate constant for $\mathrm{CD}_{2} \mathrm{CO}$ dissociation as a function of the photolysis energy. The $C O(v=0, J=12)$ PHOFEX curve $(\Delta t=1.7 \mu \mathrm{s})$ is shown in the reaction threshold region; its intensity is arbitrarily scaled. The solid line is a RRKM fit.

tunneling for any reasonable value for the imaginary rapidly than could be consistent with one-dimensional frequency for a barrier of the height observed. At this level of detail a quantum mechanical treatment of the reaction coordinate coupled to at least one other coordinate appears to be needed. Ultimately, it will be valuable to compare such a theory with rates for fully resolved initial rotational states.

The $a b$ initio calculations for the geometry and vibrational frequencies of ketene at the transition state have proven to be extremely useful in the interpretation of experimental results. The zero-point energies for the transition state measured from the difference between the observed thresholds for $\mathrm{CH}_{2} \mathrm{CO}$ and $\mathrm{CD}_{2} \mathrm{CO}$ is in exact agreement with that predicted, Figure 1-2. The impulsive model calculation based on the $a b$ initio transition state geometry predicted the $\mathrm{CO}$ product rotational distribution quantitatively thus confirming the $a b$ initio $\mathrm{CCO}$ angle of $116^{\circ}$. The scaled $a b$ initio value for the $\mathrm{C}-\mathrm{C}-\mathrm{O}$ bend of the

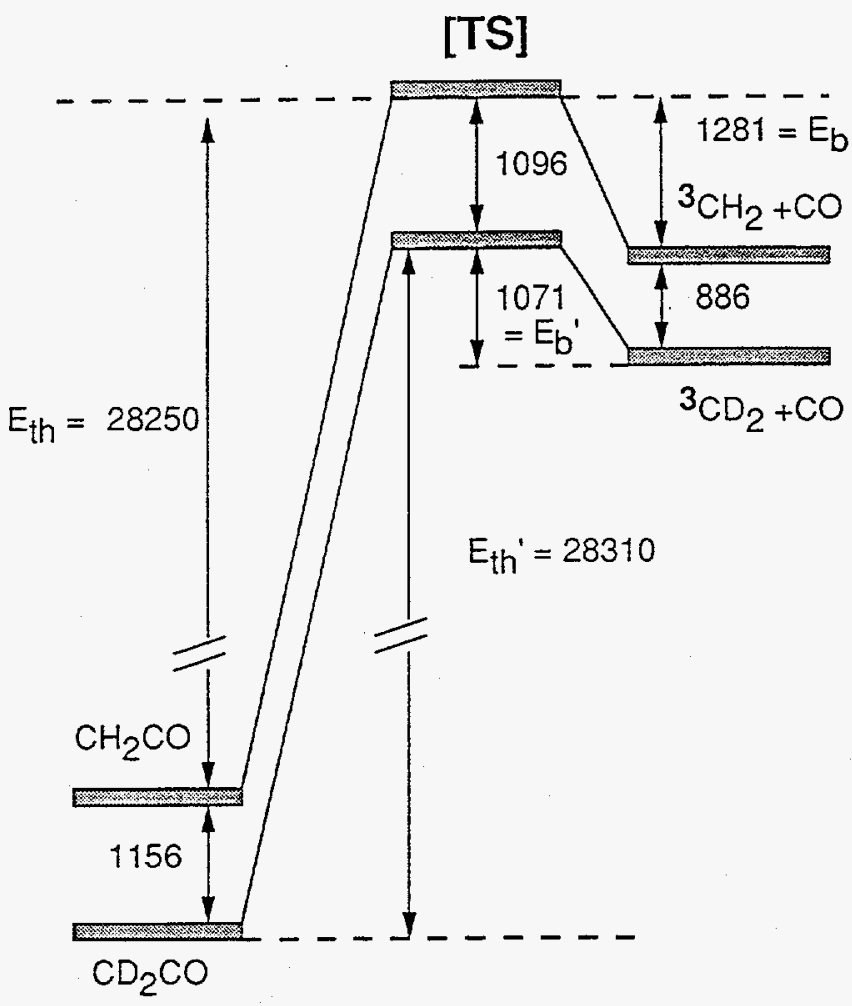

Figure 1-2. Energy diagram for the dissociation of $\mathrm{CH}_{2} \mathrm{CO}$ and $\mathrm{CD}_{2} \mathrm{CO}$ on the triplet surface. The energy differences $\left(\mathrm{cm}^{-1}\right)$ among the zero-point energy levels are shown.

$\mathrm{CH}_{2} \mathrm{CO}$ transition state, $252 \mathrm{~cm}^{-1}$, is in excellent agreement with the value of $250 \pm 15 \mathrm{~cm}^{-1}$ found here. The theoretical frequency derives further support from the observed width of the $\mathrm{CO}$ rotational distribution. The $\mathrm{CH}_{2}$ wagging frequency at the $\mathrm{CH}_{2} \mathrm{CO}$ transition state appears to be $20 \%$ lower than calculated. The barrier for the hindered internal rotation of ketene at the transition state is found to be $240 \mathrm{~cm}^{-1}$ when a sinusoidal potential function, Figure 1-3, is assumed. This is $35 \%$ lower than the best $a b$ initio value of $384 \mathrm{~cm}^{-1}$. Similarly, the barrier height for the reaction itself, $3.06 \mathrm{kcal} / \mathrm{mol}$, is a $\mathrm{kcal}$ or so less than the best theoretical estimate. Thus the actual transition state is found to be somewhat looser than predicted by the $a b$ initio calculations.

The density of vibrational states of the excited reactant and the number of strongly coupled triplet levels, $g_{t}$, control the step height in the RRKM fits to measured rate constants. The Whitten-Rabinovitch approximation gives a good estimation for the ratio of the densities of $\mathrm{CH}_{2} \mathrm{CO}$ and $\mathrm{CD}_{2} \mathrm{CO}$ reactant states. The absolute values for the density of states for $\mathrm{CH}_{2} \mathrm{CO}$ and $\mathrm{CD}_{2} \mathrm{CO}$ are found to be $1.11 \mathrm{~g}_{\mathrm{t}}$ and $1.19 \mathrm{~g}_{\mathrm{t}}$ times higher than predicted by the WhittenRabinovitch approximation for energies between 28,200 


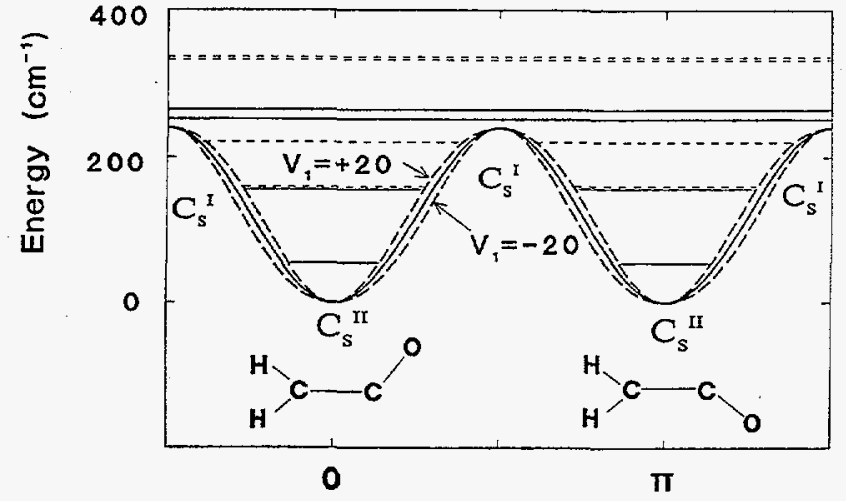

Internal Rotation Angle (0)

Figure 1-3. The model potential function for the hindered internal rotor, $\mathrm{V}(\theta)=(1 / 2) \mathrm{V}_{0}(1-\cos 2 \theta)+(1 / 2) \mathrm{V}_{1}(1-\cos 4 \theta)$, where $\mathrm{V}_{0}=240 \mathrm{~cm}^{-1}$ and $\mathrm{V}_{1}=0$ (solid line) and $\pm 20 \mathrm{~cm}^{-1}$. The ab initio $\mathrm{C}_{\mathrm{s}} \mathrm{I}^{\mathrm{I}}$ transition-state geometry is drawn. For $\mathrm{C}_{\mathrm{s}}{ }^{\mathrm{I}} \mathrm{O}$ is out of the plane with the $\mathrm{CCO}$ plane bisecting the $\mathrm{HCH}$ angle. The calculated energy levels listed are shown for $\mathrm{K}=0$.

and $28,700 \mathrm{~cm}^{-1}$. Recent $a b$ initio work on $S_{0}$ ketene gives densities of states in the range of 1-1.5 times the harmonic density. This would suggest that $g_{t}$ is closer to 1 than to 3 .

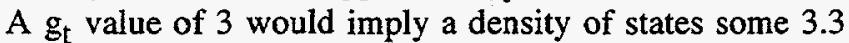
times larger than harmonic. Those few molecules for which experimental data exist, exhibit even greater excesses in density of states, five to ten times harmonic. Thus there is no firm basis on which to select values for $g_{t}$ and $\rho(E)$.

It is clear from comparison of the experimental data with RRKM theory that there is significantly more to be learned about the dynamics of passage through the transition state. A quantum mechanical treatment of the dynamics including coupling of the reaction coordinate to the coupled rovibrational degrees of freedom orthogonal to it will be necessary. For this treatment to be meaningfully compared with experiment, the $a b$ initio potential energy surface must be mapped out through the full spread of energetically accessible bond angles near the transition state. In order to interpret experiments in which the initial rotational and nuclear spin states are fully selected, $a b$ initio results are needed for the direction of the transition dipole moment vector for the UV excitation and for the magnitudes of the intersystem crossing matrix elements. Ultimately, the shapes of steps, their dependence on $\mathrm{J}$ and $\mathrm{K}$, and the symmetries of transition-state vibrational levels along with accurate dynamical calculations will provide a clear picture of molecular dynamics in the region of the transition state.
2. Quantum Yield for Production of $\mathrm{CH}_{3} \mathrm{NC}$ in the Photolysis of $\mathrm{CH}_{3} \mathrm{NCS}$ (Publication 2)

\section{R.A. Alvarez and C.B. Moore}

Methyl isothiocyanate $\left(\mathrm{CH}_{3} \mathrm{NCS} \equiv \mathrm{MITC}\right)$ is widely used in agriculture as a soil fumigant in various formulations. Due to its high vapor pressure, $(\sim 13$ torr at $297 \mathrm{~K})$ MITC can enter the troposphere by spray drift during application or subsequent volatilization. MITC concentrations of up to $3 \mathrm{mg} \mathrm{m}^{-3}$ have been measured at sites downwind from fields injected with metham-sodium, which decomposes in the soil to yield MITC. The fate of species in the troposphere is determined by some combination of (1) photodissociation; (2) chemical removal, e.g., reaction with other trace species such as $\mathrm{OH}$, $\mathrm{O}_{3}$, and $\mathrm{NO}_{3}$; and (3) physical removal, e.g., diffusion to the stratosphere or rainout. The persistence of pollutants in the atmosphere may pose health and environmental risks. In order to assess the potential risks arising from the use of toxic substances, it is useful to know the dominant mechanism and the rate for their removal from the atmosphere. This study, which deals with the fundamental photochemistry of MITC, indicates that solar photodissociation could be the chief removal pathway of MITC.

The ultraviolet (uv) and vacuum-ultraviolet (vuv) absorption spectra of MITC have been previously reported, but the spectrum at wavelengths with significant solar flux $(1 \geq 295 \mathrm{~nm})$ has not been measured. The NCS radical in various electronic states has been detected as a product from the uv and vuv photolysis of MITC. The decomposition of MITC in a discharge has been observed to yield NCS, CS, CN, and $S_{2}$. The production of $S\left({ }^{3} \mathrm{P}\right)$ atoms and $\mathrm{CH}_{3} \mathrm{NC}$ in solutions of MITC irradiated with $254 \mathrm{~nm}$ light has also been reported to occur, with a maximum yield of $45 \%$. However, nothing is known about the photolysis of MITC at energies corresponding to the long-wavelength tail of its lowest energy absorption band, and the quantum yields of the photolysis products have not been quantified at any wavelength.

The absorption cross section, s, of MITC in the solar uv is found here to be significant. The MITC absorption band extends out to at least $340 \mathrm{~nm}$, but the wavelength interval $305-330 \mathrm{~nm}$ accounts for $86 \%$ of the total rate for MIC absorption of the actinic flux in the troposphere. For a photodissociation quantum yield of 1.0 , the total absorption rate of $6.7 \times 10^{-6} \mathrm{~s}^{-1}$ yields a lifetime with respect to photolysis of 41 hours, for July 1 , noon sunlight at a latitude of $40^{\circ} \mathrm{N}$. Thus photochemical degradation in 
the atmosphere could be an effective removal pathway, if the photodissociation quantum yield were sufficiently high. This possibility is explored by quantitatively measuring the yield of photolysis products.

Using an FTIR spectrometer, methyl isocyanide, $\mathrm{CH}_{3} \mathrm{NC}$, was observed to be the major stable product formed in pure gas phase MITC samples (2-10 torr) photolyzed with a $308 \mathrm{~nm}$ excimer laser. The yield of $\mathrm{CH}_{3} \mathrm{NC}$ was then examined using a diode laser flash kinetic spectrometer. The $\mathrm{CH}_{3} \mathrm{NC}$ production can be described by the combination of a direct photolytic component and a secondary bimolecular channel, reactions 1 and 2 , respectively.

$$
\begin{gathered}
\mathrm{CH}_{3} \mathrm{NCS}+\mathrm{hn}(308 \mathrm{~nm}) \rightarrow \mathrm{CH}_{3} \mathrm{NC}+\mathrm{S}\left({ }^{3} \mathrm{P}\right) \\
\mathrm{S}\left({ }^{3} \mathrm{P}\right)+\mathrm{CH}_{3} \mathrm{NCS} \rightarrow \mathrm{CH}_{3} \mathrm{NC}+\mathrm{S}_{2}
\end{gathered}
$$

From biexponential fits to the $\mathrm{CH}_{3} \mathrm{NC}$ rise, it is estimated that reactions 1 and 2 each contributes approximately 50\% of the observed signal. The rate constant $\mathrm{k}_{2}$ was measured to be $(2.0 \pm 0.3) \times 10^{-11} \mathrm{~cm}^{3}$ molecule ${ }^{-1} \mathrm{~s}^{-1}$ by fitting a single exponential to the slow component of the $\mathrm{CH}_{3} \mathrm{NC}$ rise over the MITC pressure range of $0.092-0.32$ torr. S atoms were scavenged by an olefin to measure the yield of reaction (1) alone. The $\mathrm{CH}_{3} \mathrm{NC}$ rise is prompt, and the $\mathrm{CH}_{3} \mathrm{NC}$ yield is $0.98 \pm 0.24$. The yield is not decreased by addition of $1 \mathrm{~atm}$ of $\mathrm{N}_{2}$. The results indicate that solar photolysis of MITC is an effective pathway, and perhaps the major pathway, for its removal from the atmosphere.

\section{Work in Progress}

Unimolecular reaction studies on triplet ketene are being initiated with full rotational state resolution in the initial excitation. An IR optical parametric oscillator has been set up to select a single excited rovibrational state that will then be further excited to the reaction threshold by a uv laser pulse. Quantitative theoretical analysis of the resonances in the isotopic exchange rate is being carried out by William Miller's group with a multidimensional reaction coordinate. Ultimately these resonances will be studied experimentally with complete rotational resolution.

Reaction rates for radical-radical reactions are being measured. Infrared spectra and chemical reaction kinetics of intermediates in organometallic photochemistry in gas and liquid phase are being studied jointly with R.G. Bergman and C.B. Harris. Emphasis is on $\mathrm{CH}$ activation chemistry. Studies of $\mathrm{CH}$ activation systems in liquid $\mathrm{Kr}$ and $\mathrm{Xe}$ are proceeding well.

\section{FY 1994 PUBLICATIONS AND REPORTS}

\section{Refereed Journals}

1. R.A. Alvarez and C.B. Moore, "Absolute Yields of $\mathrm{CO}$, $\mathrm{CO}_{2}$ and $\mathrm{H}_{2} \mathrm{CO}$ from the Reaction $\mathrm{CH}_{2}\left(\tilde{\mathrm{X}}^{3} \mathrm{~B}_{1}\right)+\mathrm{O}_{2}$ by IR Diode Laser Flash Kinetic Spectroscopy," J. Phys. Chem. 98, 174 (1994); LBL-34762.

2. R.A. Alvarez and C.B. Moore, "Quantum Yield for Production of $\mathrm{CH}_{3} \mathrm{NC}$ in the Photolysis of $\mathrm{CH}_{3} \mathrm{NCS}$," Science 263, 205 (1994); LBL-34995.

3. R.H. Schultz, A.A. Bengali, M.J. Tauber, B.H. Weiller, E.P. Wasserman, K.R. Kyle, C.B. Moore, and R.G. Bergman, "IR Flash Kinetic Spectroscopy of C-H Bond Activation of $\mathrm{d}_{0}$ - and $\mathrm{d}_{12}-\mathrm{Cyclohexane}$ by $\mathrm{Cp} * \mathrm{Rh}(\mathrm{CO})_{2}$ in Liquid Rare Gases: Kinetics, Thermodynamics, and an Unusual Isotope Effect," J. Am. Chem. Soc. 116, 7369 (1994); LBL-35543.

4. A.A. Bengali, R.H. Schultz, C.B. Moore, and R.G. Bergman, "Activation of the $\mathrm{C}-\mathrm{H}$ Bonds in Neopentane and Neopentane- $d_{12}$ by $C p * R h(C O)_{2}$ : Spectroscopic and Temporal Resolution of Rhodium-Krypton and RhodiumAlkane Complex Intermediates and an Unusual Equilibrium Isotope Effect," J. Am. Chem. Soc. 116, 9585 (1994); LBL35544.

\section{LBL Reports}

5. A.A. Bengali, R.G. Bergman, and C.B. Moore, "Evidence for the Intervention of Free 16-electron Species Rather than Solvate Complexes in the Ultraviolet Irradiation of $\mathrm{CpCo}(\mathrm{Co})_{2}$ in Liquefied Noble Gas Solvents," J. Am. Chem. Soc. (submitted); LBL-36638.

6. A.A. Bengali, B.A. Arndtsen, P.M. Burger, R.H. Schultz, B.H. Weiller, K.R. Kyle, C.B. Moore, and R.G. Bergman, "Activation of Carbon-Hydrogen Bonds in Alkanes and Other Organic Molecules by $\operatorname{Ir}(\mathrm{I}), \operatorname{Rh}(\mathrm{I})$, and IR(III) Complexes," Pure and Applied Chemistry (submitted); LBL-36639.

7. S.K. Kim, E.R. Lovejoy, and C.B. Moore, "Transition State Vibrational Level Thresholds for the Dissociation of Triplet Ketene," J. Chem. Phys. (in press); LBL-36226.

8. C.B. Moore, "Energy States and Energy Flow Near the Transition States of Unimolecular Reactions," in 1995 Proceedings of the Robert A. Welch Foundation, 38th Conference on Chemical Research (in press); LBL-36640.

9. R.A. Alvarez, "Infrared Diode Laser Studies of the Products from the Reaction $\mathrm{CH}_{2}\left(\tilde{\mathrm{X}}{ }^{3} \mathrm{~B}_{1}\right)+\mathrm{O}_{2}$ and from the NearUV Photolysis of $\mathrm{CH}_{3} \mathrm{NCS}$," Ph.D. Thesis, University of California, Berkeley, December 1993; LBL-34918.

\section{Invited Talks}

10. C.B. Moore, "Energy Levels and Dynamics at the Transition State of Unimolecular Reactions," Physical Chemistry Seminar, Department of Chemistry, University of California, Los Angeles, CA, January 31, 1994. 
11. C.B. Moore, "Transition States and Energy Transfer in the Unimolecular Reactions of Ketene," American Chemical Society National Meeting, San Diego, CA, March 1994.

12. C.B. Moore, "A Spectroscopic View of Quantized Motions at Unimolecular Transition States," American Physical Society Meeting, Pittsburgh, PA, March 1994.

13. C.B. Moore, "A Spectroscopic View of Quantized Motions at Unimolecular Transition States," Opening Plenary Lecture, XXIst Informal Conference on Photochemistry, York University, Toronto, Ontario, Canada, May 1994.

14. C.B. Moore, "Selective Photochemistry," DOE Combustion Research Meeting, Tahoe City, CA, June 1-3, 1994.

15. C.B. Moore, "Reform of Undergraduate Chemistry Education at UCB," Conference of the California
Association of Chemistry Teachers, Lawrence Berkeley Laboratory, Berkeley, CA, August 1994.

16. C.B. Moore, "Quantized Motions for Transition States of Unimolecular Reactions," Physical Chemistry Seminar, University of California, Berkeley, CA, August 1994.

17. C.B. Moore, "Dynamics of Unimolecular Transition States," 13th International Symposium on Gas Kinetics, University College, Dublin, Ireland, September 1994.

18. C.B. Moore, "Energy States and Energy Flow Near the Transition States of Unimolecular Reactions," Robert A. Welch Foundation Conference, Houston, TX, October 24 $25,1994$. 


\section{Photodissociation of Free Radicals*}

\author{
Daniel M. Neumark, Investigator
}

\section{INTRODUCTION}

While many photodissociation studies of stable molecules have been performed in recent years, it has proved difficult to extend these experiments to studies of reactive free radicals. This is largely due to the difficulty of implementing a clean, well-characterized source of free radicals. We have developed a novel approach to this problem by setting up an experiment in which free radicals are generated by photodetachment of a mass-selected anion beam, rather than the more conventional strategies in which radicals are formed by photolysis of a stable precursor or by a chemical reaction. Since nearly all radicals have a positive electron affinity, this approach should be quite general.

In the experiment, an $8 \mathrm{keV}$ beam of cold massselected anions is photodetached with a pulsed laser. The resulting neutral radicals are photodissociated with a second pulsed laser, and the photofragments are detected with high $(-50 \%)$ efficiency using a microchannel plate detector that lies about $100 \mathrm{~cm}$ downstream from the photodissociation laser. The center of the detector is blocked so that the undissociated radicals do not impinge on it, but the photofragments move off the beam axis and strike the detector. The experiment can be operated in several modes. We can measure the total photofragment signal as a function of dissociation laser wavelength, thereby mapping out the dissociative electronic transitions of the radical. We can measure the time-of-flight distribution of the photofragments, thereby obtaining an approximated kinetic energy distribution at a fixed photodissociation wavelength. Finally, using a two-particle position and time sensing detector, we can determine detailed photofragment energy and angular distributions.

In many cases we can obtain the radical bond dissociation energy from the maximum kinetic energy release in this experiment, and this typically yields an accurate value for the heat of formation of the radical. This thermodynamic information is critical in assessing the possible role of the radical in complex chemical mechanisms, such as combustion or atmospheric chemistry. From the details of the kinetic energy and angular distributions, one learns about the symmetry and lifetime of the excited state (or states) of the radical that lead to photodissociation, as well as how the energy during dissociation is distributed among the photofragment degrees of freedom.

1. Study of the Predissociation of $\mathrm{CH}_{3} \mathrm{O} \tilde{\mathrm{A}}\left({ }^{2} \mathrm{~A}_{1}\right)$ by Fast Beam Photofragment Translational Spectroscopy (Publication 10)

\section{D.L. Osborn, D.J. Leahy, E.M. Ross, and D.M. Neumark}

The dissociation dynamics of the methoxy radical $\left(\mathrm{CH}_{3} \mathrm{O}\right)$ following excitation of $\tilde{\mathrm{A}}\left({ }^{2} \mathrm{~A}_{1}\right) \leftarrow \tilde{\mathrm{X}}\left({ }^{2} \mathrm{E}\right)$ spectroscopy. The radicals are produced from photodetachment of a fast, cold negative ion beam. Three product channels are identified: (1) $\mathrm{CH}_{3}+\mathrm{O}$, (2) $\mathrm{CH}_{2}+$ $\mathrm{OH}$, and (3) $\mathrm{CH}_{2} \mathrm{O}+\mathrm{H}$, with channel (1) by far the dominant channel. In channel (1), resolved vibrational structure of the methyl fragment is seen in the translational energy distribution, providing insight into the dissociation dynamics of $\mathrm{CH}_{3} \mathrm{O}$. Several thermodynamics quantities are also obtained: $\mathrm{D}_{0}\left(\mathrm{CH}_{3}-\mathrm{O}\right)=87.8 \pm 0.3 \mathrm{kcal} / \mathrm{mol}, \Delta H_{\mathrm{f}, 0}^{\circ}$ $\left(\mathrm{CH}_{3} \mathrm{O}\right)=6.8 \pm 0.4, \mathrm{D}_{0}\left(\mathrm{CH}_{3} \mathrm{O}-\mathrm{H}\right)=104.0 \pm 0.5, \Delta \mathrm{H}_{\text {acid }, \mathrm{O}}$ $\left(\mathrm{CH}_{3} \mathrm{O}-\mathrm{H}\right)=381.4 \pm 0.7$, and $\mathrm{D}_{0}\left(\mathrm{H}-\mathrm{CH}_{2} \mathrm{O}\right)=19.8 \pm 0.4$.

2. Observation of the Correlated $\mathrm{O}^{3} \mathrm{P}_{\mathrm{j} 1},{ }^{3} \mathrm{P}_{\mathrm{j} 2}$ State Distribution from the Predissociation of $\mathrm{O}_{2} \mathrm{~B}^{3} \Sigma_{\mathrm{u}}^{-}$ (Publication 1) ${ }^{\dagger}$

\section{D.J. Leahy, D.R. Cyr, $\neq$ D.L. Osborn, and D.M. Neumark}

We present a fast beam photofragment translational spectroscopy study of the predissociation of the $\mathrm{O}_{2} \mathrm{~B}{ }^{3} \Sigma_{\mathrm{u}}^{-}$ state. The $B^{3} \Sigma_{\mathrm{u}}^{-}\left(v^{\prime}=7\right) \rightarrow \mathrm{X}^{3} \sum_{\mathrm{g}}^{-}\left(v^{\prime \prime}=4\right)$ transition of the Schumann-Runge band is then excited, resulting in predissociation to two $\mathrm{O}^{3} \mathrm{P}_{\mathrm{j}}$ atoms. We determine the photofragment kinetic energy and angular distribution using a time- and position-sensitive detector. Our energy resolution $(10 \mathrm{meV})$ is sufficient to resolve the energy splittings of the $O$ atom spin-orbit levels, enabling us to determine the correlated $\left(\mathrm{j}_{1}, \mathrm{j}_{2}\right)$ fine structure distribution for the photofragments. These results do not appear to be consistent with predictions based on a recent model of $\mathrm{O}_{2} \mathrm{~B}$ $3 \sum_{\mathrm{u}}^{-}$state predissociation.

\footnotetext{
This work is also supported in part by the National Science Foundation, Grant CHE91-08145.

${ }^{\ddagger}$ Sandia National Laboratories, P.O. Box 969, MS-9055, Livermore, CA 94550 .
}

*This work was supported by the Director, Office of Energy Research, Office of Basic Energy Sciences, Chemical Sciences Division of the U.S. Department of Energy under Contract No. DE-AC03-76SF00098. 


\section{Work in Progress}

We have completed a detailed study of the predissociation dynamics of $\mathrm{O}_{2}$ in which various rovibrational transitions in the $\mathrm{O}_{2} \mathrm{~B}^{3} \Sigma_{\mathrm{u}}^{-} \leftarrow \mathrm{X}^{3} \Sigma_{\mathrm{g}}^{-}$band (the Schumann-Runge band) are excited. The upper state predissociates to two $\mathrm{O}{ }^{3} \mathrm{P}$ atoms, and we have measured the correlated fine structure distribution for the two $O$ atoms starting from the first twelve vibrational levels of the $\mathrm{O}_{2} \mathrm{~B}$ state. We find dramatic differences in this distribution as a function of vibration, indicating that the predissociation mechanism changes markedly with vibrational level. An analysis of these results using both adiabatic and diabatic models shows that non-adiabatic effects at long-range play an important role in the predissociation dynamics. The analysis also suggests that interference effects due to predissociation of a single level by multiple repulsive states are likely to occur. Studies of complex radicals such as phenoxy $\left(\mathrm{C}_{6} \mathrm{H}_{5} \mathrm{O}\right)$ and vinoxy $\left(\mathrm{C}_{2} \mathrm{H}_{3} \mathrm{O}\right)$ are currently under way. Preliminary results on phenoxy indicate that both visible and ultraviolet excitation lead only to the lowest energy channel, $\mathrm{C}_{5} \mathrm{H}_{5}+\mathrm{CO}$. The vinoxy radical has several relatively low energy dissociation channels, namely $\mathrm{H}+\mathrm{C}_{2} \mathrm{H}_{2} \mathrm{O}, \mathrm{CH}_{3}+\mathrm{CO}$, and $\mathrm{CH}_{2}+\mathrm{HCO}$. The competition between these channels and the detailed dynamics of each channel will provide considerable insight into the nature of the low-lying excited state potential energy surfaces of this radical. We are also interested in investigating the photodissociation of the $\mathrm{ClO}_{2}$ radical. There has been some controversy in the literature about the branching ratio between the major $\mathrm{ClO}+\mathrm{O}$ and minor $\mathrm{Cl}+\mathrm{O}_{2}$ channel. With our detection scheme, this branching ratio can be determined in a more straightforward way than in previous investigations.

\section{FY 1994 PUBLICATIONS AND REPORTS}

\section{Refereed Journals}

1. D.J. Leahy, D.R. Cyr, D.L. Osborn, and D.M. Neumark, "Observation of the Correlated $\mathrm{O}^{3} \mathrm{P}_{\mathrm{j} 1},{ }^{3} \mathrm{P}_{\mathrm{j} 2}$ State Distribution from the Predissociation of $\mathrm{O}_{2} \mathrm{~B}^{3} \Sigma_{\mathrm{u}}$ Chem. Phys. Lett. 216, 503 (1993); LBL-34820.

2. C.C. Arnold and D.M. Neumark, "Threshold Photodetachment (ZEKE) Spectroscopy of $\mathrm{Si}_{3}-$," J. Chem. Phys. 100, 1797 (1994).

3. D.W. Arnold, C. Xu, E.H. Kim, and D.M. Neumark, "Study of Low-Lying Electronic States of Ozone by Anion Photoelectron Spectroscopy of $\mathrm{O}_{3}^{-}$," J. Chem. Phys. 101, 912 (1994).

4. M.J. Davis, H. Koizumi, G.C. Schatz, S.E. Bradforth, and D.M. Neumark, "Experimental and Theoretical Study of the
$\mathrm{O}+\mathrm{HCl}$ Transitions State Region by Photodetachment of OHCl,"' J. Chem. Phys. 101, 4708 (1994).

5. C. Xu, E. de Beer, D.W. Arnold, C.C. Arnold, and D.M. Neumark, "Anion Photoelectron Spectroscopy of Smail Indium Phosphide Clusters $\left(\operatorname{In}_{x} \mathrm{P}_{y}^{-} ; x, y,=1-4\right)$," J. Chem. Phys. 101, 5406 (1994).

6. Y. Zhao, G. Reiser, I. Yourshaw, C.C. Arnold, and D.M. Neumark, "Study of $\mathrm{ArBr}^{-}, \mathrm{Arl}^{-}$, and $\mathrm{KrI}^{-}$Anions and the Corresponding Neutral van der Waals Complexes by Anion Zero Electron Kinetic Energy Spectroscopy," J. Chem. Phys. 101, 912 (1994).

\section{Other Publications}

7. C.C. Arnold and D.M. Neumark, "Study of Small Carbon and Silicon Clusters using Negative Ion Photodetachment Techniques," in Advances in Metal and Semiconductor Clusters, Vol. III, ed. M. A. Duncan (JAI Press, Greenwich, CT, 1994).

8. D.M. Neumark, "Studies of Transient Species Using Negative Ion Photodetachment," in Proceedings of The Robert A. Welch Foundation, 38th Conference on Chemical Research, Chemical Dynamics of Transient Species, (Robert A. Welch Foundation, Houston, TX, 1995).

\section{LBL Reports}

9. D.R. Cyr, "Fast Beam Studies of Free Radical Photodissociation," (Ph.D. Thesis), LBL-34924.

10. D.L. Osborn, D.J. Leahy, E. M. Ross, and D.M. Neumark, "Study of the Predissociation of $\mathrm{CH}_{3} \mathrm{O} \tilde{\mathrm{A}}\left({ }^{2} \mathrm{~A}_{1}\right)$ by Fast Beam Photofragment Translational Spectroscopy," J. Chem. Phys. (in press); LBL-36222.

\section{Invited Lectures}

11. D.M. Neumark, "Studies of Clusters using ZEKE Spectroscopy on Anions," Optical Society of America Interdisciplinary Laser Science Conference, Toronto, Ontario, Canada, October 1993.

12. D.M. Neumark, "Transition State Spectroscopy of the $\mathrm{F}+\mathrm{H}_{2}$ Reactions," University of Wisconsin, Madison, WI, ACS Speaker, February 1994.

13. D.M. Neumark, "Fast Beam Studies of Free Radical Photodissociation," Workshop on Imaging Methods in Molecular Structure and Dynamics, Neve Ilan, Israel, June 1994.

14. D.M. Neumark, "Transition State Spectroscopy of the $\mathrm{F}+\mathrm{H}_{2}$ and $\mathrm{OH}+\mathrm{H}_{2}$ Reaction," Hebrew University, Jerusalem, Israel, June 1994

15. D.M. Neumark, "Fast Beam Studies of Free Radical Photodissociation," FORTH IESL, Crete, Greece, June 1994.

16. D.M. Neumark, "Studies of the Vibrational Spectroscopy of Clusters Using Negative Ion Photodetachment," Gordon Conference on Vibrational Spectroscopy, Wolfeboro, NH, August 1994. 
17. D.M. Neumark, "Non-Adiabatic Effects in Free Radical Photodissociation," American Chemical Society National Meeting, Washington, DC, August 1994.

18. D.M. Neumark, "Studies of Semiconductor Clusters by Negative Ion Photodetachment," Seventh International Symposium on Small Particles and Inorganic Clusters, Kobe, Japan, September 1994.
19. D.M. Neumark, "Studies of Clusters by Negative Ion Photodetachment," Conference on Dynamical Aspects of Clusters, Himeji, Japan, September 1994.

20. D.M. Neumark, "Studies of Semiconductor Clusters by Negative Ion Photodetachment," 1994 Spectroscopy Conference on The Future of Spectroscopy: From Astronomy to Biology, Sainte-Adele, Quebec, Canada, September 1994. 


\section{Physical Chemistry with Emphasis on Thermodynamic Properties*}

\author{
Kenneth S. Pitzer, Investigator
}

\section{INTRODUCTION}

The purpose of this program is the discovery and development of methods of calculation of thermodynamic and related properties of important chemical systems by the use of quantum and statistical mechanics together with experimental measurements for key systems. Current emphasis is on fluid systems that include ionic or strongly dipolar components in novel ranges of conditions, including near-critical and supercritical temperatures and compositions extending from pure water or other polar solvent to pure fused salt. Recently, there has been great interest in the exact pattern of behavior of ionic fluids near the critical point. This is expressed by critical exponents. The results reported below constitute major progress in resolution of this problem. Earlier advances yielded improved equations for electrolyte solutions that are now being applied to a wide variety of systems of industrial or geological interest, including geothermal brines. Other recent research involved the development of a feasible method of relativistic quantum mechanical calculation for molecules containing very heavy atoms and its application to important examples. This method is now in wide use.

\section{Behavior of Ionic Fluids Near the Critical Point; Critical Exponents (Publication 1)}

\section{T. Narayanan and K.S. Pitzer}

The near-critical behavior of fluids of neutral molecules is well established. Their limiting critical exponents are those of an Ising lattice with $\beta=0.325$ for density or composition below $T_{c}$ and $\gamma=1.24$ for compressibility or light scattering above $T_{c}$. Recent investigations of ionic fluids, however, show classical (mean field) exponents $(\beta=0.50, \gamma=1.00)$ near, but not too near, the critical point but do not exclude a crossover to Ising exponents very close to $T_{\mathrm{c}}{ }^{1}$ The current research involves light scattering (turbidity) measurements just above $T_{c}$ for systems where ionic forces are present but of intermediate strength as compared to nonionic forces.

\footnotetext{
*This work was supported by the Director, Office of Energy Research, Office of Basic Energy Sciences, Chemical Sciences Division, of the U.S. Department of Energy under Contract No. DE-AC03-76SF00098.
}

More precisely, we report the critical behavior of osmotic compressibility $\left(\chi_{\mathrm{T}}\right)$, that was deduced from turbidity, for two ionic fluid mixtures. The salt is tetrabutylammonium picrate in each case. The measurements covered the reduced temperature, $t$, range $7 \times 10^{-5} \leq \mathrm{t} \leq 7 \times 10^{-2}$. The mechanism underlying the liquid-liquid phase separation is, predominantly, Coulombic in one case and solvophobic for the other. We find that in the Coulombic case the phase separation shows an unambiguous crossover from a mean field $(\gamma=1.0)$ to an Ising ( $\gamma=1.24)$ critical exponent, rather than a true mean field exponent, as the critical temperature $\left(T_{c}\right)$ is approached (Figure 1-1). We find, however, that in a highdielectric fluid, where the ionic forces are weak but solvophobic forces cause phase separation, Ising exponents are observed.

1. K.S. Pitzer, Acc. Chem. Res. 23, 333 (1990); J.M.H. Levelt Sengers and J.A. Given, Mol. Phys. 80, 899 (1992).

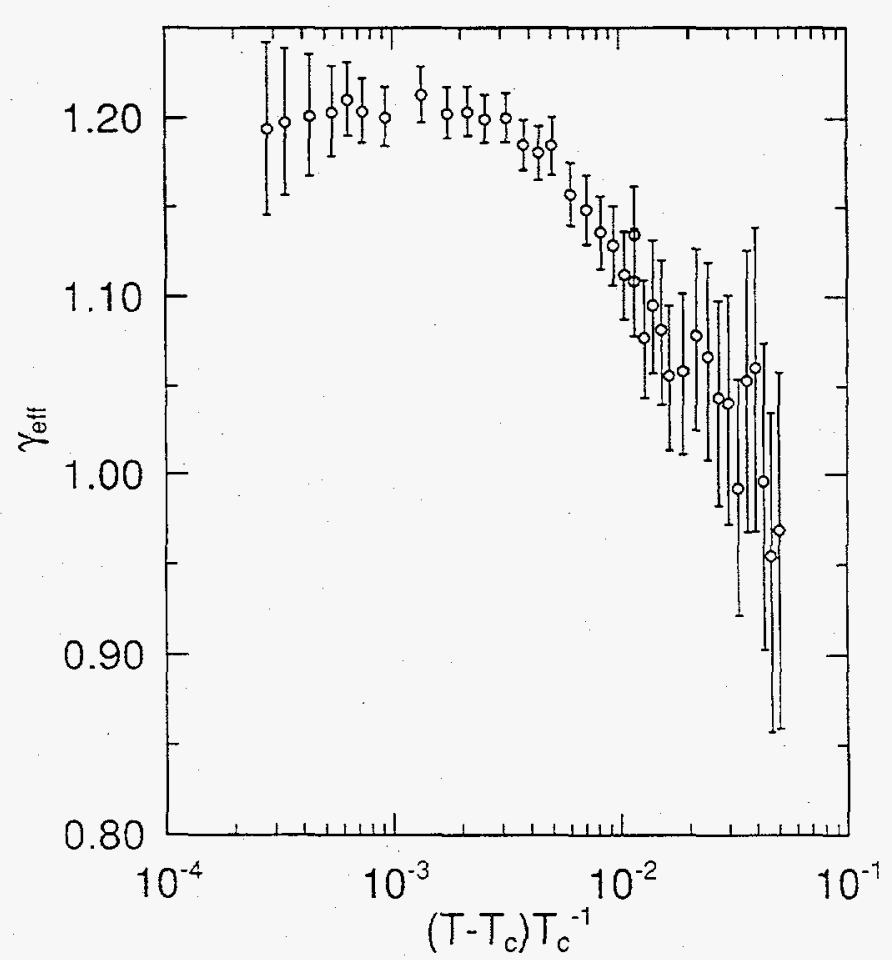

Figure 1-1. Effective exponents $\gamma_{\text {eff }}$ for the strongly ionic system showing the crossover from 1.24 at very small $t=\left(T-T_{c}\right) / T_{c}$ to 1.0 at larger $\mathrm{t}$. 
2. Thermodynamic Properties of $0-6 \mathrm{~mol} \mathrm{~kg}^{-1}$ Aqueous Sulfuric Acid from 273.15 to $328.15 \mathrm{~K}$ (Publication 2)

\section{S.L. Clegg, ${ }^{\dagger}$ J.A. Rard, ${ }^{\ddagger}$ and K.S. Pitzer}

Aqueous sulfuric acid is extremely important in many respects. Generalized equations are presented for an extended form of the Pitzer molality-based thermodynamic model, involving an ionic strength-dependent third virial coefficient. Compatibility with the established formulation is retained.

Osmotic coefficients, emf measurements, degrees of dissociation of the $\mathrm{HSO}_{4}^{-}$ion, differential enthalpies of dilution and heat capacities for aqueous $\mathrm{H}_{2} \mathrm{SO}_{4}$ from 273.15 to $328.15 \mathrm{~K}, 0-6.1 \mathrm{~mol} \mathrm{~kg}^{-1}$ and at $1 \mathrm{~atm}$ pressure have been critically evaluated. Treating this solution as the mixture $\mathrm{H}^{+}-\mathrm{HSO}_{4}^{-}-\mathrm{SO}_{4}^{2}-\mathrm{H}_{2} \mathrm{O}$, and using hydrogen sulfate dissociation constants from the literature, the model parameters were fitted to the data yielding a self-consistent representation of activities, speciation and thermal properties together with the standard potentials of four electrochemical cells and standard-state heat capacities of the $\mathrm{SO}_{4}^{2-}$ ion as functions of temperature. The model equations represent the experimental data accurately (without the use of mixture parameters $\theta_{\mathrm{HSO}_{4}, \mathrm{SO}_{4}}$ and

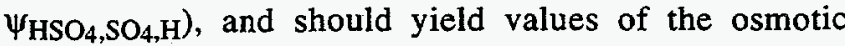
coefficient that are suitable for use as an isopiestic standard over this temperature and molality range. The new model will also enable improved prediction of the properties of mixed acidic sulfate systems.

\footnotetext{
${ }^{\dagger}$ School of Environmental Sciences, University of East Anglia, Norwich, UK NR4 7TJ.

${ }^{\ddagger}$ Lawrence Livermore National Laboratory, University of California, Livermore, CA 94550.
}

\section{Work in Progress}

The very-near-critical studies of ionic fluids will continue for part of FY 1995 with measurements on systems using the same ionic salt but different solvents of systematically varied dielectric constant $\varepsilon$. The shift in location of the crossover with change in $\varepsilon$ will confirm the general picture and provide a severe test for theory for this type of system.

Research is starting on equation-of-state representation of high-temperature aqueous systems involving quadrupolar molecules. The initial and an important real example is $\mathrm{CaCl}_{2}-\mathrm{H}_{2} \mathrm{O}$. In the range of relatively low $\varepsilon$, the solute is the linear $\mathrm{Cl}-\mathrm{Ca}-\mathrm{Cl}$ molecule with very large quadrupole moment. As an initial step, dipole-quadrupole mixtures with hard-sphere repulsion are being simulated in
Monte Carlo calculations for comparison with an existing theory using a Pade approximation. Then the research will proceed to consider the experimental data for $\mathrm{CaCl}_{2}-\mathrm{H}_{2} \mathrm{O}$ in the range above $523 \mathrm{~K}$.

\section{FY 1994 PUBLICATIONS AND REPORTS}

\section{Refereed Journals}

1. T. Narayanan and K.S. Pitzer, "Critical Behavior of Ionic Fluids,” J. Phys. Chem. 98, 9170 (1994); LBL-35246.

2. S.L. Clegg, J.A. Rard, and K.S. Pitzer, "Thermodynamic Properties of $0-6 \mathrm{~mol} \mathrm{~kg}^{-1}$ Aqueous Sulfuric Acid from 273.15 to $328.15 \mathrm{~K}$," J. Chem. Soc. Faraday Trans. 90, 1875 (1994); LBL-34579.

3. K.S. Pitzer and S.M. Sterner, "Equations of State Valid Continuously from Zero to Extreme Pressures for $\mathrm{H}_{2} \mathrm{O}$ and $\mathrm{CO}_{2}$," J. Chem. Phys. 101, 3111 (1994); LBL-35321. ${ }^{\dagger}$

4. C.S. Oakes, R.J. Bodnar, J.M. Simonson, and K.S. Pitzer, "Critical and Supercritical Properties for 0.3 to $3.0 \mathrm{~mol} \cdot \mathrm{kg}^{-1}$ $\mathrm{CaCl}_{2}$ (aq)," Geochim. et Cosmochim. Acta 58, 2421 (1994); LBL-34907. ${ }^{\dagger}$

5. K.S. Pitzer and C.S. Oakes, "Thermodynamics of Calcium Chloride in Concentrated Aqueous Solutions and in Crystals," J. Chem. Eng. Data 39, 553 (1994); LBL-34806. ${ }^{\dagger}$

6. B.S. Krumgalz, R. Pogorelsky, Ya.A. Iosilevskii, A. Weiser, and K. S. Pitzer, "Ion Interaction Approach for Volumetric Calculations for Solutions of Single Electrolytes at $25^{\circ} \mathrm{C}$," J. Solution Chem. 23, 849 (1994); LBL-35473. ${ }^{\dagger}$

7. S.M. Sterner and K.S. Pitzer, "An Equation of State for Carbon Dioxide Valid from Zero to Extreme Pressures," Contributions to Mineralogy and Petrology 117, 362 (1994); LBL-35490. ${ }^{\dagger}$

\section{LBL Reports}

8. T. Narayanan and K.S. Pitzer, "Mean-Field to Ising Crossover in Ionic Fluids," Phys. Rev. Lett. (in press); LBL35416.

9. T. Narayanan and K.S. Pitzer, "Turbidity of a Near Critical Ionic Fluid," Proceedings of the 12th Symp. on Thermophysical Properties, Boulder, CO, June 19-24, 1994, Int. J. Thermophys. (in press); LBL-35519.

10. C.S. Oakes, R. J. Bodnar, J.M. Simonson, and K.S. Pitzer, " $\mathrm{CaCl}_{2}-\mathrm{H}_{2} \mathrm{O}$ in the Supercritical and Two-phase Ranges," Proceedings of the 12th Symp. on Thermophysical Properties, Boulder, CO, June 19-24, 1994, Int. J. Thermophys. (in press); LBL-35515.

11. K.S. Pitzer and S.M. Sterner, "Equations of State Valid Continuously from Zero to Extreme Pressures with $\mathrm{H}_{2} \mathrm{O}$ and $\mathrm{CO}_{2}$ as Examples," Proceedings of the 12th Symp. on Thermophysical Properties, Boulder, CO, June 19-24, 1994, Int. J. Thermophysics (in press); LBL-35354. ${ }^{\dagger}$

12. B.S. Krumgalz, R. Pogorelskii, and K.S. Pitzer, "Volumetric Properties of Single Aqueous Electrolytes from Zero to 
Saturation Concentration at $25^{\circ} \mathrm{C}$ Represented by Pitzer's Ion-interaction Equations," J. Phys. Chem. Ref. Data (submitted); LBL-35668. ${ }^{\dagger}$

13. K.S. Pitzer and S. Jiang, "Equation of State for $\mathrm{NaCl}-\mathrm{H}_{2} \mathrm{O}$; Comparison with Mineral Dehydration Equilibria," Contributions to Mineralogy and Petrology (submitted); LBL-36205.

\section{Invited Talks}

14. K.S. Pitzer, "Thermodynamics and Phase Relations of $\mathrm{NaCl}-\mathrm{KCl}-\mathrm{H}_{2} \mathrm{O}$ and the Aqueous Binaries," 1994 Spring
Meeting of the American Geophysical Union, Baltimore, MD, May 1994.

15. K.S. Pitzer, "Thermodynamics of Industrial and Natural Waters," Symposium on Basic Chemistry for Industrial Use, American Chemical Society, 208th National Meeting, August 1994.

\footnotetext{
This work was supported by the Director, Office of Energy Research, Office of Basic Energy Sciences, Division of Engineering and Geosciences of the U.S. Department of Energy under Contract No. DEAC03-76SF00098.
} 


\section{ATOMIC PHYSICS}

\section{Search for the Electric Dipole Moment of the Electron*}

\author{
Eugene D. Commins, Investigator
}

\section{INTRODUCTION}

Does the electron possess an electric dipole moment (EDM)? This has become one of the most interesting and important questions in modern physics. Existence of an EDM would imply that time reversal $(\mathrm{T})$ invariance and parity $(\mathrm{P})$ are violated, and the question is related to the well-known phenomenon of $\mathrm{CP}$ violation in neutral kaon decay, which is known to be equivalent to $T$ violation. No satisfactory theoretical explanation for $\mathrm{CP}$ violation exists, but several theoretical models of that phenomenon have been proposed, with diverse predictions for the electron EDM. In particular, during the past year one model now widely favored by elementary particle theorists has emerged: "supersymmetric grand unification."1 It predicts that the value of the electron electric dipole moment is closely related to the branching ratio for the forbidden decay $\mu \rightarrow \mathrm{e} \gamma$. During the last year we completed the second version of our experiment and published our latest experimental result ${ }^{2}$ :

$\mathrm{d}_{\mathrm{e}}($ expt $)=$

$[1.8 \pm 1.2$ (statistical) \pm 1.0 (systematic) $] \cdot 10^{-27} \mathrm{e} \mathrm{cm}$

It has been shown ${ }^{1}$ that the result given in Eq. (1) represents a very critical test of supersymmetric grand unified theories, a test of greater significance than a variety of much more expensive experimental efforts, such as second-generation proton decay searches.

Furthermore, the motivation to improve the present experimental limit is extremely high. If after an improvement of precision by another factor of ten, one were still to obtain a null result for $d_{e}$, this would present great difficulties for supersymmetric grand unified theories. Conversely, if supersymmetric grand unification is the right theoretical approach, we should expect to discover clear evidence for the electron EDM. Naturally this has generated intense interest in experimental searches for $d_{e}$ among a number of groups around the world. However, we believe that the third and latest version of our experiment, construction of which began in earnest during the past year,

*This work was supported by the Director, Office of Energy Research, Office of Basic Energy Sciences, Chemical Sciences Division, of the U.S. Department of Energy under Contract No. DE-AC03-76SF00098. offers the best hope for a sound and reliable measurement, with sensitivity at or near $10^{-28} \mathrm{e} \mathrm{cm}$.

1. R. Barbieri, L. Hall, and A. Strumia, LBL-36381.

2. E.D. Commins, S.B. Ross, D. DeMille, and B.C. Regan, Phys. Rev. A50, 2960 (1994).

\section{Summary of the Completed Experiment}

To detect the presence of an electric dipole moment $\mathbf{d}$, one must place the particle of interest in an electric field $\mathbf{E}$ and measure its incremental energy: $W=-\mathbf{d} \cdot \mathbf{E}$. This obviously cannot be done directly with a free (charged) electron. One is thus led to the following question: If an unpaired valence electron in a neutral paramagnetic atom were to possess an EDM $d_{e}$, would the atom as a whole possess an EDM $d_{a}$ proportional to $d_{e}$ ? At first glance this would appear to be impossible, since a neutral atom is not accelerated when placed in a unform external electric field. Thus the average force on each of the charges must be zero. Since in the nonrelativistic limit the force on a charge is proportional to the local electric field, this would seem to imply that the average electric field at each charge in the atom is zero. The external $\mathbf{E}$ field would therefore appear to have been screened by rearrangement (polarization) of the other charges.

However this argument, which may be cast in quantum mechanical form, is valid only in the nonrelativistic limit. It has been shown that when relativistic effects are taken into account $d_{a}$ can actually be quite large for suitable paramagnetic atoms. The ratio $R=d_{a} / d_{e}$ is found to be $\Rightarrow Z^{3} \alpha^{2}$ in magnitude (where $Z$ is the atomic number and $\alpha$ is the fine structure constant). ${ }^{3}$ The "enhancement factor" $R$ has by now been calculated quite accurately for a number of atoms. For the ground $6^{2} \mathrm{P}_{1 / 2}$ state of thallium $(\mathrm{Z}=81)$ one finds

$$
R=-585
$$

with an uncertainty of about $5 \% .^{2}$ In the present experiment, a search is made for $\mathrm{d}_{\mathrm{a}}\left({ }^{205} \mathrm{Tl}, 6^{2} \mathrm{P}_{1 / 2}\right)$ and the results are interpreted in terms of $d_{e}$ by means of Eq. (2).

The atomic beam magnetic resonance method is employed with separated oscillating fields, using ${ }^{205} \mathrm{Tl}$ atoms in the ${ }^{6} \mathrm{P}_{1 / 2}$ ground state (see Figure 1-1). A magnetic field $\mathbf{B}$ defines the axis of quantization, and an electric field $\mathbf{E}$, nominally parallel to $\mathbf{B}$, is applied in the region between the oscillating fields. Laser optical pumping is used for state selection and analysis, and the signal is fluorescence accompanying the decay of excited atoms in the analyzer region. The signature of a nonzero electric 


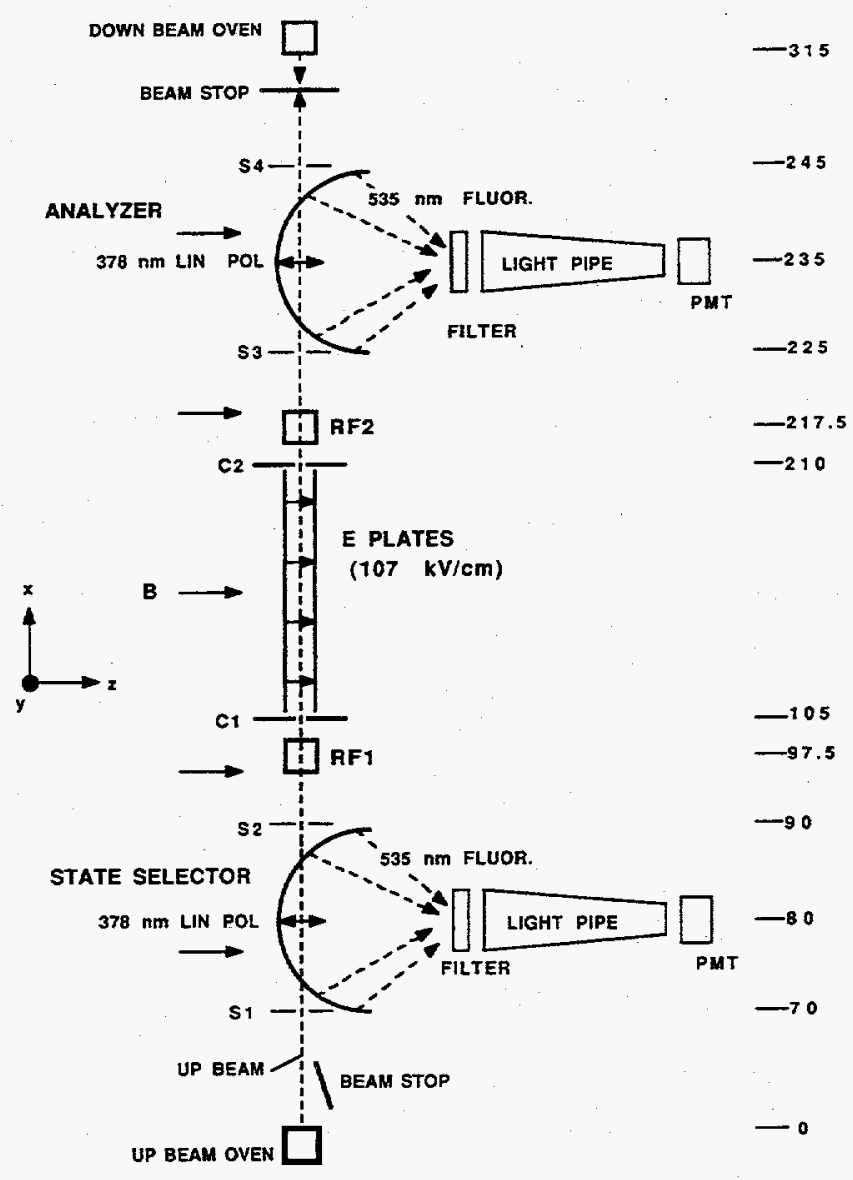

Figure 1-1. Schematic diagram of experimental apparatus used to achieve latest published EDM results. S1-4 and C1,2 are slits used for collimating the atomic beams and diagnosing systematic effects. The numbers at the right refer to distances in centimeters from the up beam oven slit to $S 1$, center of state selector, etc.

dipole moment is a dependence of the signal on the P,T-odd rotational invariant $\mathbf{E} \cdot \mathbf{B}$. Two counterpropagating atomic beams are employed to reduce greatly a systematic effect due to the motional magnetic field Exv/c. Auxiliary experiments are performed to isolate and eliminate residual sources of systematic error, among them an effect due to Berry's geometric phase. ${ }^{3}$

i. P.G.H. Sandars, Phys. Lett. 14, 194 (1965); 22, 190 (1966).

2. Z. W. Liu and H.P. Kelly, Phys. Rev. A45, R4210 (1992).

3. E.D. Commins, Am. J. Phys. 59, 1077 (1991).

\section{Outline of the New Electron EDM Experiment}

We have carried out a detailed analysis of the various sources of noise and systematic error leading to the uncertainties quoted in result in Eq. (1) [see Introduction above]. Every one of these will be improved very substantially in the new experimental arrangement now under construction and nearing completion (see Figure 2-1). Like its predecessor, it utilizes the atomic beam magnetic resonance method with separated oscillating fields, and in fact will be housed in the same vacuum chambers and utilize much of the same equipment. However, in the new experiment we employ two parallel atomic beams going upward, and two counterpropagating atomic beams going downward. Furthermore, each atomic beam consists simultaneously of thallium atoms in the ground $6 \mathrm{P}_{1 / 2}$ state and sodium atoms in the ground $3 \mathrm{~S}_{1 / 2}$ state. A magnetic field $B$ defines the axis of quantization, and equal and opposite electric fields $\mathbf{E},-\mathbf{E}$ for the parallel beams are applied in the region between the oscillating fields and are nominally parallel to B. As previously, laser optical pumping is used for state selection and analysis, and the signal is fluorescence accompanying the decay of excited atoms in the analyzer region. However, in the new experiment, there are separate state selection and analyzer regions for each parallel beam and for thallium (sodium).

By employing two parallel beams from the same oven simultaneously, we shall acquire data simultaneously for $\mathbf{E}$, $-\mathbf{E}$ and thus essentially eliminate one very major source of noise in the old experiment: temporal fluctuations in the magnetic field $\mathbf{B}$. By utilizing sodium as well as thallium, we shall reduce by at least an order of magnitude the

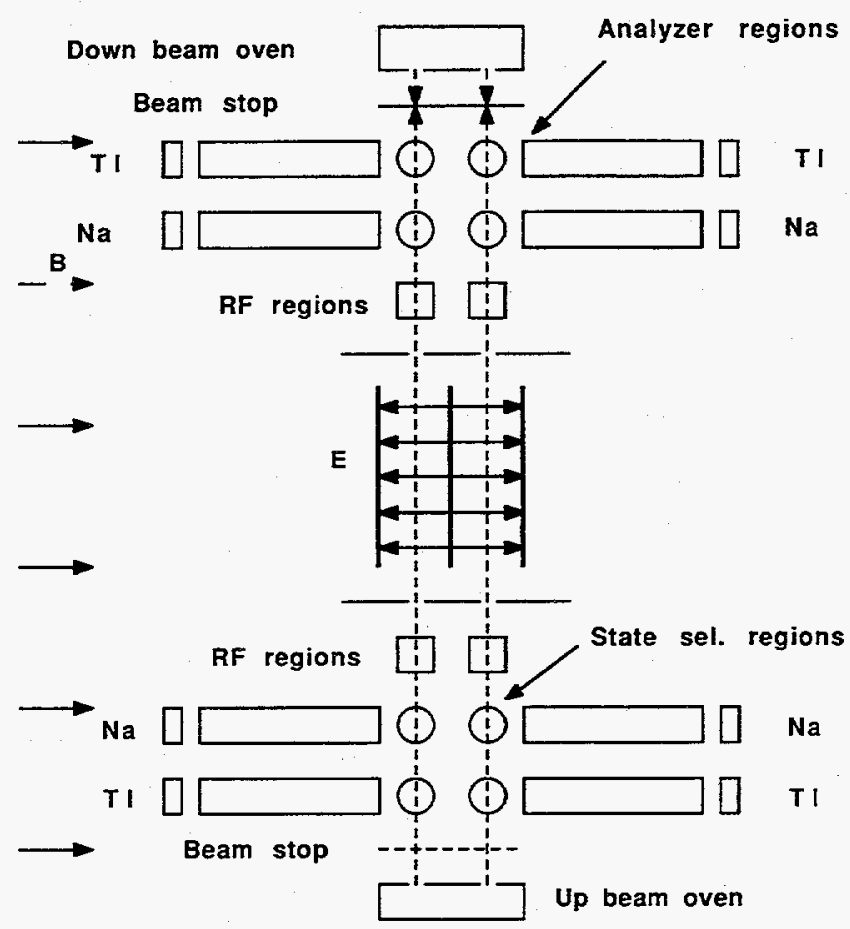

Figure 2-1. Schematic diagram of the new EDM experiment presently being constructed. 
residual sources of systematic error due to geometric phase, the $\mathbf{E} \times \mathbf{v} / \mathrm{c}$ effect, and residual magnetic effects due to leakage and charging currents associated with the electric field. This is because sodium exhibits all of these systematic effects, in some cases much more sensitively than thallium, while its enhancement factor is extremely small: $R \approx 0.3$, so that it exhibits essentially no EDM effect. Thus sodium acts as an ideal calibration. Furthermore, a variety of improvements are being installed to make the new experiment more sensitive than the old one, including more sensitive signal detection, better electric field plates with the possibility to go to higher electric fields, and more advanced and sophisticated computer control.

The construction phase of the new experiment is nearing completion, and we are nearly ready to begin preliminary observations.

\section{High Precision Measurement of Parity Nonconservation in Atomic Ytterbium}

\section{E.D. Commins, D. DeMille, and S. Freedmant}

Precise measurements of atomic parity nonconservation (PNC) provide stringent tests of the standard model of electroweak interactions, with uncertainties usually dominated by imprecise knowledge of atomic structure. It has been noted ${ }^{1}$ that measurements of atomic PNC in a chain of isotopes of the same atom would be useful, since atomic structure uncertainties cancel in the ratio of the PNC effect for different isotopes. In combination with precise high-energy electroweak measurements, isotopic ratio measurements would provide an unambiguous test for the existence of new "tree-level" interactions such as new $Z$ bosons. ${ }^{2-3}$ The interpretation of isotopic ratio measurements in terms of the standard model would at present be limited by the knowledge of nuclear neutron distributions. For a reasonable range of neutron numbers in a heavy element, one finds an uncertainty of $\sim 1 \%$ in the determination of $\sin ^{2} \vartheta_{\mathrm{W}}$ (where $\vartheta_{\mathrm{W}}$ is the Weinberg angle)..$^{3-4}$ This is already at the level of the limits from the best single isotope measurements, due to atomic structure uncertainties; moreover future measurements of neutron distributions by other means may improve this situation. ${ }^{3}$

We have found $\mathrm{d}^{5}$ an extremely favorable case for isotopic PNC comparisons: the $\left(6 \mathrm{~s}^{2}{ }^{1} \mathrm{~S}_{0} \rightarrow 6 s 5 \mathrm{~d}{ }^{3} \mathrm{D}_{1}\right)$ transition $(408 \mathrm{~nm})$ in atomic $\mathrm{Yb}(\mathrm{Z}=70)$ (see Figure 3-1). This transition has a very large PNC E1 amplitude ${ }^{5-6}$ : $\left(\operatorname{Im}\left(E 1_{\mathrm{PNC}}\right)>1.1 \cdot 10^{-9} \mathrm{e} \mathrm{a}_{0}\right)$, which arises because of the

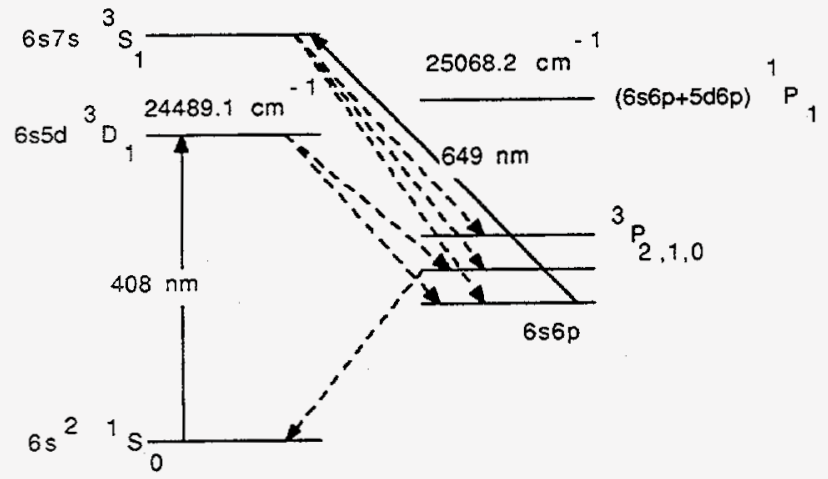

Figure 3-1. Relevant energy levels, excitation wavelengths, and fluorescent decay channels of atomic $\mathrm{Yb}$.

small energy separation between the $6 s 5 d{ }^{3} D_{1}$ state and the odd parity state $(6 s 6 p+5 d 6 p){ }^{1} \mathbf{P}_{1}$. (In fact, this amplitude is approximately 100 times as large as the analogous amplitude for the $6 \mathrm{~S} \rightarrow 7 \mathrm{~S}$ transition in cesium, which has been utilized in the most precise PNC experiment to date.) In addition, the $408 \mathrm{~nm}$ transition in $\mathrm{Yb}$ has a strongly suppressed M1 amplitude $\left(\approx 10^{-4} \mu_{B}\right)$ and a Stark-induced E1 amplitude of convenient size; hence the transition may be studied conveniently by the well-known technique of Stark-PNC interference. ${ }^{7-8}$ Furthermore, $\mathrm{Yb}$ has a wide range of stable isotopes, and the isotope shifts are large enough to resolve all individual isotopes by conventional laser spectroscopic methods.

Thus we are planning an experiment on the $408 \mathrm{~nm}$ transition in $\mathrm{Yb}$ analogous to the very successful cesium PNC experiment at Boulder ${ }^{7}$ and also similar in some ways to an earlier successful PNC experiment on thallium in this laboratory. ${ }^{8}$ So far, no funds from the Chemical Sciences Division have been used for this new project, and the only funding presently available is a one-time grant of $\$ 100 \mathrm{k}$ from LBL and the UC Berkeley Physics Dept. to be used for equipment and operating costs.

\footnotetext{
From Nuclear Science Division and his group.

1. V.A. Dzuba, V.V. Flambaum, and I.B. Khriplovich, Z. Phys. D1, 243 (1986).

2. M. E. Peskin and T. Takeuchi, Phys. Rev. D46, 381 (1992).

3. S.J. Pollock, E.N. Fortson, and L. Wilets, Phys. Rev. C46, 2587 (1992); E.N. Fortson, Y. Pang, and L. Wilets, Phys. Rev. Lett. 65, 2857 (1990).

4. B.Q. Chen and P. Vogl, Phys. Rev. C48, 1392 (1993).

5. D. DeMille, Phys. Rev. Lett., to be published.

6. M. Kozlov, private communication.

7. M.S. Noecker, B.P. Masterson, and C. E. Wieman, Phys. Rev. Lett. $61,310(1988)$

8. P. Drell and E.D. Commins, Phys. Rev. A32, 2196 (1985).
} 
FY 1994 PUBLICATIONS AND REPORTS

\section{Refereed Journals}

1. E.D. Commins, S.B. Ross, D. DeMille, and B.C. Regan, "Improved Experimental Limit on the Electric Dipole Moment of the Electron, Phys. Rev. A50, 2960 (1994).

\section{Other Publications}

2. S.B. Ross, "Search for the Electric Dipole Moment of the Electron," Ph.D. Thesis, University of California, Berkeley, CA, 1993.

3. D.P. DeMille, "High Precision Measurement of Parity Nonconservation in Atomic Yb," Phys. Rev. Lett. (in press). 


\section{High-Energy Atomic Physics*}

Harvey A. Gould, Investigator

\section{INTRODUCTION}

The goals of this program are (1) to achieve an understanding of the physics of electron capture from electron-positron pair production, and heavy particle capture from pair production-this is to be done by performing both experiments and calculations-and (2) to search for a charge-parity violating permanent electric dipole moment (EDM) of the electron as small as $10^{-30} \mathrm{e}-$ $\mathrm{cm}$ or smaller-thousands of times smaller than the present limit.

Recent results include the discovery of a new atomic collision process, electron capture from pair production: the process in which an electron-positron pair is produced by the transient electromagnetic field of a relativistic ion-atom collision and the electron from the pair emerges from the collision bound to the ion. Capture from pair production is predicted to be an important beam loss mechanism at the Relativistic Heavy Ion Collider (RHIC).

Present activities include (1) extending the measurement of electron capture from pair production to $10 \mathrm{GeV} /$ nucleon collision energies and the capture of particles heavier than electrons, (2) performing calculations of capture from pair production using massively parallel computing, and (3) constructing a new experiment to search for an electron EDM using laser trapping and cooling.

\section{Measurement of Projectile Energy and Atomic Number Dependence of Electron Capture from Pair Production in Relativistic Heavy Ion Collisions (Publication 1)}

\section{A. Belkacem, H. Gould, B. Feinberg, R. Bossingham, and W. Meyerhof ${ }^{t}$}

We have previously reported the first observation and measurement of electron capture from electron-positron pair production. Electron capture from pair production has unique properties. Its cross section is expected to increase with collision energy and it can take place between two bare ions. As a result, it is expected to be the dominant

\footnotetext{
*This work was supported by the Director, Office of Energy Research, Office of Basic Energy Sciences, Chemical Sciences Division, of the U.S. Department of Energy under Contract No. DE-AC03-76SF00098.
}

capture mechanism at highly relativistic energies and may limit the lifetime of stored beams of colliding bare ions in colliders such as the RHIC and LHC.

We report the first experimental evidence that the cross section for electron capture from pair production process does increase with increasing relativistic collision energy. As shown in Figure 1-1, the rate of increase is about the same as for electron-positron pair production without the capture of the electron, indicating that the fraction of captured electrons remains roughly constant over the energy range from $0.4 \mathrm{GeV} / \mathrm{u}$ to $1.3 \mathrm{GeV} / \mathrm{u}$.

The experiment was performed at the Lawrence Berkeley Laboratory's Bevalac, using $0.405 \mathrm{GeV} / \mathrm{u}, 0.956$ $\mathrm{GeV} / \mathrm{u}$, and $1.3 \mathrm{GeV} / \mathrm{u} \mathrm{La}^{57+}$ beams incident on thin $\mathrm{Au}$, $\mathrm{Ag}$, and $\mathrm{Cu}$ targets. The experimental setup and apparatus is the same as previously described for a $\mathrm{U}^{92+}$ beam.

Combining with uranium data reported previously gives a projectile atomic number dependence for 0.956 $\mathrm{GeV} / \mathrm{u}$ ions on a Au target of $Z_{\mathrm{p}} 6.54 \pm 0.65$ for capture from pair production and $Z_{p}{ }^{1.53 \pm 0.80}$ for the free pair production process. The projectile atomic number dependence for capture from pair production is larger than the $Z_{p}{ }^{5}$ from the simple perturbation theory, where as the free pair dependence is consistent with the perturbation theory result.

Measurements will be extended to $10 \mathrm{GeV} /$ nucleon to determine if the cross section for capture from pair production continues to rise as rapidly (as the free pair cross section): a question that has important implications for heavy-ion colliders. An experiment to look for muon capture from pair production is being developed.

${ }^{\dagger}$ Stanford University, Stanford, CA 94305.

2. Work in Progress: Nonperturbative MomentumSpace Calculation of Atomic Collision Processes at Relativistic Energies

\section{A. Belkacem, K. Momberger, and A. Sørensen ${ }^{\dagger}$}

We are developing a new nonperturbative method to calculate probabilities of atomic processes involved in relativistic heavy-ion collisions. This method is based on a direct numerical integration of the time dependent Dirac equation in momentum space. The momentum space description has a number of advantages: (i) unlike configuration space, the total wave function stays localized around the origin at all times; (ii) the projection on the highly localized continuum states is simple; (iii) no problem of incompleteness of the basis set is encountered as for the coupledchannels method; (iv) high collision energies are tractable.

Within the Lorentz gauge, the Dirac equation for the electron spinor $\psi(k, t)$ takes the following form in momentum space: 

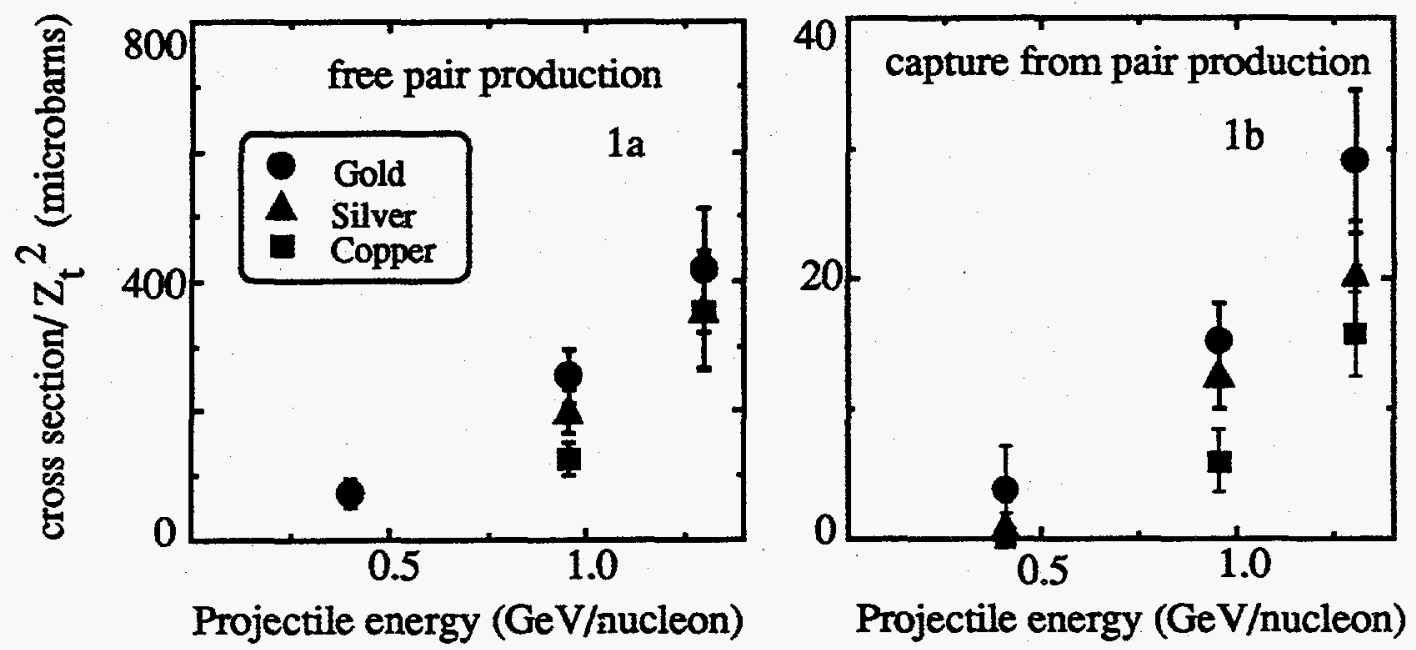

Figure 1-1. Cross sections (divided by $\mathrm{Z}_{4}^{2}$ ) for free pair production ( $1 \mathrm{a}$ ) and electron capture from pair production (lb) for $\mathrm{La}^{57+}$ incident on $\mathrm{Au}, \mathrm{Ag}$, and $\mathrm{Cu}$ targets. The capture cross sections (1b) are corrected for accidental events.

$$
\begin{aligned}
& \mathrm{i} \frac{\partial}{\partial \mathrm{t}} \psi(\mathbf{k}, \mathrm{t})=(\alpha \mathbf{k}+\beta) \psi(\mathbf{k}, \mathrm{t}) \\
& \quad+\frac{\mathrm{e}^{2}}{2 \pi^{2}} \int \mathrm{d}^{3} \mathrm{k}^{\prime}\left(\frac{Z_{t}}{\mathrm{q}^{2}}+\frac{Z_{\mathrm{p}}(1-\alpha \mathbf{v})}{\mathrm{q}_{\mathrm{x}}^{2}+\mathrm{q}_{y}^{2}+\mathrm{q}_{z}^{2} / \gamma^{2}} \mathrm{e}^{\mathrm{iq} \cdot \mathbf{R}(\mathrm{t})}\right) \Psi\left(\mathbf{k}^{\prime}, \mathrm{t}\right)
\end{aligned}
$$

where $\mathbf{q}=\mathbf{k}-\mathbf{k}$. We applied the straight-line trajectory approximation, that is, we assume a point-like projectile of charge $Z_{p}$ e moving along a rectilinear path $R(t)=v_{t} e_{z}+b_{x}$ at constant velocity $\mathbf{v}=\mathbf{v e}_{\mathrm{z}}$ with impact parameter $\mathrm{b}$ relative to point-like target nucleus of charge $Z_{\mathrm{t}} e$ at rest at the origin. $\alpha$ and $\beta$ are the usual Dirac matrices and $\gamma=(1-$ $\left.v^{2}\right)^{-1 / 2}$ the usual Lorentz factor. We use units where $\hbar=$ $\mathrm{m}=\mathrm{c}=1$.

We applied the method to a bare $\mathrm{Au}^{79+}$ projectile impinging with $\gamma=2$ and $b=0.5$ on a hydrogen-like $U^{91+}$ ion assumed initially in a $1 \mathrm{~s}$ spin up state.

We solved the above integro-differential equation using the PVM software on a farm of 10 Sparc stations. The final time-evolved wave function contains a wealth of information on different atomic processes such as pair creation with capture, $\mathrm{K}$-shell ionization, excitation, and electron transfer to the projectile. The probabilities can be obtained by the projection of the final $\psi(k, t)$ on the corresponding states. For example, making use of time reversal, the projection of $\psi(k, t)$ on the negative energy continuum states gives probabilities for pair production with capture of the electron to a $1 \mathrm{~s}$ state (we obtain here $0.10 \%$ ). We observe that a substantial fraction of the density stays in the initial state throughout the collision (ending at $32.8 \%$ ). The probability of excitation of the target amounts to $11.7 \%$ and the inner shell ionization (projection on the continuum positive states) amounts to $56 \%$.
The computer code corresponding to the above calculation will be modified to run on a massively parallel computer (Maspar). We will extend the calculation to several impact parameters in order to deduce a cross section that can be compared to the experimental results.

\footnotetext{
Permanent Address: Institute of Physics and Astronomy, Aarhus . University, DK-8000 Aarhus C, Denmark.
}

\section{Work in Progress: Electron EDM Measurement by Trapping and Cooling of Francium}

We are constructing an experiment to search for a permanent (EDM) of the electron using laser trapping and cooling of francium. Compared to standard atomic beam experiments, this method greatly reduces the major systematic effects and increases the sensitivity of the experiment. An experimental limit of $10^{-30} \mathrm{e}-\mathrm{cm}$-thousands of times smaller than the current upper limit-may be reached. As first step Cs has been trapped and cooled and a fountain is under construction. A steady supply of francium has been obtained as a decay product of ${ }^{229} \mathrm{Th}$, eliminating the need to use an accelerator to produce francium.

\section{FY 1994 PUBLICATIONS AND REPORTS}

\section{Refereed Journals}

1. A. Belkacem, H. Gould, B. Feinberg, R. Bossingham, and W.E. Meyerhof, "Projectile Energy and Atomic Number Dependence of Electron Capture from Pair Production in 
Relativistic Heavy Ion Collisions," Phys. Rev. Lett. 73, 2432 (1994); LBL-36311.

\section{LBL Reports}

2. A. Belkacem, H. Gould, B. Feinberg, R. Bossingham, and W.E. Meyerhof, "Measurement of Electron Capture from $\mathrm{e}^{+}-\mathrm{e}^{-}$Pair Production by $0.956 \mathrm{GeV} / \mathrm{u} \mathrm{U}^{92+}$ on $\mathrm{Au}, \mathrm{Ag}, \mathrm{Cu}$ and Mylar Targets," invited paper at the XVIII ICPEAC, Aarhus, Denmark, Conference Proceedings 295, edited by T. Andersen, B. Fastrup, F. Folkmann, H. Knudsen, and N. Andersen (AIP Press, New York, 1993), p. 849; LBL34534.

3. H. Gould, A. Belkacem, B. Feinberg, R. Bossingham, and W.E. Meyerhof, "Measurement of Electron Capture from Electron-Positron Pair Production," invited paper at the 9th High Energy Heavy Ion Study, Lawrence Berkeley Laboratory, Berkeley CA, Oct. 25-29, 1993; in Proceedings of the 9th High Energy Heavy Ion Study," edited by A.D. Chacon, M. Justice, and H.G. Ritter, p. 113; LBL-35984.

4. H. Gould, A. Belkacem, B. Feinberg, R. Bossingham, and W.E. Meyerhof, "Capture from Pair Production in High Temperature Collisions," in Abstracts of Contributed Papers of the Fourteenth International Conference on Atomic Physics, Boulder, CO, July 31-August 5, 1994, p. 2R-1; LBL-36761 Abs.

5. H. Gould, A. Belkacem, B. Feinberg, R. Bossingham, and W.E. Meyerhof, "Measurement of Energy- and Projectile ZDependence of Electron Capture from $\mathrm{e}^{+}-\mathrm{e}^{-}$Pair Production," Bull. Am. Phys. Soc. 39, 1105 (1994); LBL-36762.

6. B. Feinberg, A. Belkacem, H. Gould, R. Bossingham, and W.E. Meyerhof, "Capture from Pair Production as a Beam Loss Mechanism for Heavy Ions in Relativistic Colliders," Bull. Am. Phys. Soc. 39, 1169 (1994); LBL-35043.

7. K. Momberger, A. Belkacem, and A.H. Sørensen, "Nonperturbative Momentum-Space Calculation of Atomic Collision Processes at Relativistic Energies," in Abstracts of Contributed Papers of the Fourteenth International Conference on Atomic Physics, Boulder, CO, July 31August 5, 1994, p. 2R-3; LBL-36763 Abs.

8. K. Momberger, A. Belkacem, and A.H. Sørensen, "Nonperturbative Momentum-Space Approach to Relativistic Heavy Ion Collisions: Application to $\mathrm{e}^{+}-\mathrm{e}^{-}$ Pair Production with Capture," Bull. Am. Phys. Soc. 39, 1105 (1994); LBL-36764 Abs.

9. H. Gould, A. Ghiorso, and T. Dinneen, "A Laboratory Source of Francium," in Abstracts of Contributed Papers of the Fourteenth International Conference on Atomic Physics, Boulder, CO, July 31-August 5, 1994, p. ID-7; LBL-36765 Abs.

10. A. Belkacem, "Atomic Collision Processes at Relativistic Energies," in Abstracts for the IIIX International Conference on the Application of Accelerators in Research and Industry," November 7-10, 1994, edited by J.L. Duggan and I. Morgan (University of North Texas, Denton, TX), p. 58; LBL-36766 Abs.

11. H. Gould, A. Belkacem, B. Feinberg, R. Bossingham, W.E. Meyerhof, K. Momberger, A.H. Sørensen, T. Dinneen, D. McColm, and K. Street "Experiment and Theory of Relativistic Atomic Collisions," in Abstracts of the Fifteenth U.S. Department of Energy Atomic Physics Program Workshop, October 14-15, 1994, Lexington, KY; LBL36767 Abs.

\section{Invited Talks}

12. A. Belkacem, "Atomic Collision Processes at Relativistic Energies," Conference on the Application of Accelerators in Research and Industry, Denton, TX, November 8, 1994.

13. K. Momberger, "Application of Distributed and Massively Parallel Computing in Relativistic Heavy Ion Collisions," Nuclear Science Division, Lawrence Berkeley Laboratory, Berkeley, CA, October 26, 1994.

14. H. Gould, "Relativistic Atomic Physics," Dept. of Physics, University of Nevada, Las Vegas, NV, October 21, 1994.

15. H. Gould, "Relativistic Atomic Collisions," 15th U.S. DOE Atomic Physics Workshop, Lexington, KY, October 14-15, 1994.

16. H. Gould, "Electron EDM Experiment" Graduate Research Seminar, University of California, Berkeley, CA, Berkeley, August 22, 1994.

17. H. Gould, "Electron Capture from Pair Production as a Beam Loss Mechanism in Relativistic Heavy Ion Colliders," Accelerator and Fusion Research Division Beam Physics Seminar, Lawrence Berkeley Laboratory, Berkeley, CA, May 27, 1994.

18. H. Gould, "Measurements of Electron Capture from Electron-Positron Pair Production," Atomic Collisions: A Symposium in Honor of Christopher Bottcher," Oak Ridge TN, March 3-5, 1994.

19. K. Momberger, "Numerical Solution of the TimeDependent Dirac Equation in Momentum Space and Application to Atomic Processes in Relativistic Heavy-Ion Collisions," University of Kansas, Lawrence, KS, February $11,1994$. 


\section{Atomic Physics*}

Michael H. Prior, Investigator

\section{INTRODUCTION}

This program performs experimental studies of the structure and interactions of atomic systems, to provide detailed descriptions of their behavior and to challenge and stimulate theoretical understanding of the observed phenomena. Research topics are chosen that are best addressed with unique research tools and expertise available at LBL. Often topics selected have relevance to plasma behavior and diagnostics in tokamak devices or advanced laser technology. The program welcomes participation by students, and research topics are suitable for advanced degree theses.

Currently the program exploits the ability of state-ofthe-art electron cyclotron resonance (ECR) ion sources at LBL to produce intense, highly charged continuous beams for conduct of ion-atom collision, Auger, and photon spectroscopic studies. Collaborative associations with investigators from outside LBL are a means of bringing resources to the program, stimulating scientific exchange, and efficiently utilizing experimental facilities.

A prominent project within this program is currently providing the most detailed experimental description of multiple electron capture in slow $(\mathrm{v}<1$ au) ion-atom collisions. Historically, nearly all multiple electron capture studies have consisted of total cross-section measurements into unresolved or only partially resolved final states. Whereas theorists can calculate probability amplitudes for fully defined final quantum states of the products, they must sum and average over much of this detail to obtain total or partial cross sections to compare with the much less detailed experimental data. Conversely, although calculations reproduce total or partial cross-section measurements, when more detailed experimental studies of population amplitudes are available, there may be substantial disagreement at that level, and this can spur improved theoretical treatments.

\footnotetext{
* This work was supported by the Director, Office of Energy Research, Office of Basic Energy Sciences, Chemical Sciences Division of the U.S. Department of Energy under Contract No. DE-AC03-76SF00098.
}

1. Production of Low-energy Continuum Electrons at $0^{\circ}$ by $4.4 \mathrm{keV} / \mathrm{amu} \mathrm{O}^{8+}$ Ions (Publication 1 )

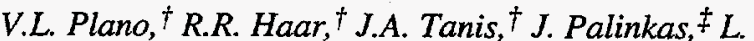

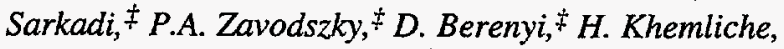 \\ M.H. Prior, and D. Schneider $\$$
}

This work reports study of a multiple electron process in which at least one low energy (few eV) electron is released from a low energy ion-atom collision while one or more are captured onto the projectile $\left(\mathrm{O}^{8+}\right)$. Much study has been devoted to the nature of double electron capture and particularly to cases where the two electrons are captured into atomic orbitals with widely differing principal quantum numbers (nonequivalent states). This has been taken as evidence of a dynamic correlation operative in the collision, which favors population of these states over those where the principal quantum numbers of the two electrons are the same (equivalent states) or nearly so. An extreme limit of the case of nonequivalent capture is that where one electron is captured to a bound state with principal quantum number $\mathrm{n}$, while the other is "captured" into the continuum just above the series limit for the two electron configuration $\mathrm{n}, \mathrm{n}^{\prime}$ with $\mathrm{n}^{\prime} \rightarrow \infty$. Thus two electrons are removed from the atomic target, one is bound to the projectile and one is free with small kinetic energy. This work extends previous studies of $\mathrm{O}^{6+}$ and $\mathrm{O}^{8+}$ in collision with $\mathrm{He}$ atoms to include $\mathrm{H}_{2}$ and $\mathrm{Ar}$ targets.

The measurements were made with slow ${ }^{18} \mathrm{O}^{8+}$ ion beams of energy $4.4 \mathrm{keV} / \mathrm{amu}$, produced by the LBL ECR (Electron Cyclotron Resonance) ion source, and transported to the experiment with the LBL/LLNL beamline facility. The beam passed through a 4-cm-long cell containing the target gas and then entered a parallel plate charge analyzer that directed selected ion product charge states onto a high rate particle multiplier. Electrons emitted from collisions in the gas cell in the direction of the projectile beam $\left(0^{\circ}\right)$ were energy analyzed by a McPherson spherical sector spectrometer with a microchannel plate, position sensitive detector at its exit. The gas cell was biased to a potential of $-200 \mathrm{eV}$ thus shifting the energy of the emitted electrons by this amount. The electrons and projectile product ions were detected in delayed coincidence utilizing a time-toamplitude converter TAC, started by the electron signal and stopped by the projectile product ion. Both the time-offlight and the position of arrival of the electron at the spectrometer exit were recorded by a computer-based collection system. Coincidence events appear as a peak in the distribution of electron/ion time-of-flight values. Data were collected for a range of gas pressures to establish single collision conditions and to determine cross sections for production of the projectile product ions. 
The observations for the $\mathrm{H}_{2}$ target are similar to those seen for $\mathrm{He}$, that is, electron/ $\mathrm{O}^{+}+$ion coincidences are seen only for $q=7$ (none for $q=8$ ), indicating the weakness of direct ionization and the existence of the two electron nature of the low energy electron production. The results for Ar target are more complex. Figure 1-1 summarizes the time-of-flight spectra obtained when the projectile charge analyzer was set for $q=8,7,6,5$. The figure shows that, again, no electrons are observed coincident with the $\mathrm{O}^{8+}$ final charge state, but there are, in addition to the two electron mechanism yielding the $q=7$ coincidences, three and four electron channels yielding the $q=6,5$ coincidences. The fraction of low energy electrons in the $q=7,6,5$ coincidences is 59,39 , and $2 \%$ respectively. Although it is possible, in a multiple electron (more than two) capture for electrons to be emitted subsequently by an Auger cascade from the projectile, it seems unlikely that this process would produce electrons in the $0-10 \mathrm{eV}$ range detected here, thus it is tempting to believe that electrons in this range can be produced directly in the collision via a mechanism analogous to that operating in the two electron

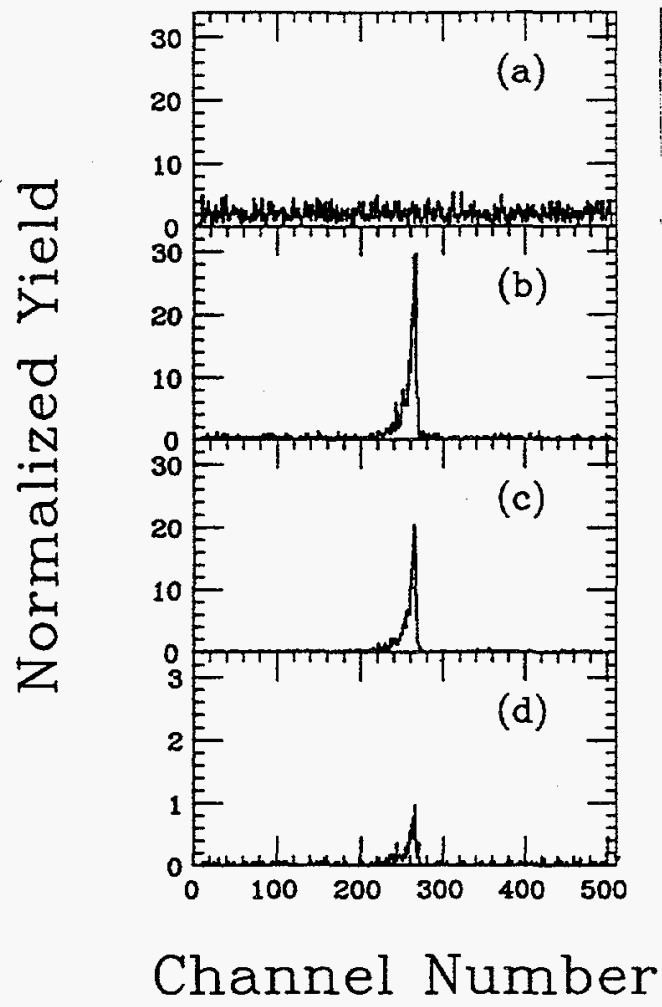

Figure 1-1. Time-of-flight spectra for coincidences between continuum electrons and outgoing projectiles for $\mathrm{O}^{8+}$ colliding with Ar. The final projectile charge states are (a) $8+$, (b) $7+$, (c) $6+$ and (d) $5+$. No coincidences are observed in (a). The signal in (d) indicates that low energy electron production occurs in transfer processes involving as many as 4 electrons. The data are normalized to the same integrated beam flux and target pressure. case, with perhaps Auger emission following. This report was made as a precursor to more complete and detailed study of these interesting multi-electron processes.

\footnotetext{
Western Michigan University, Kalamazoo, MI 49008.

$¥$ Institute of Nuclear Research (ATOMKI), Debrecen 4001, Hungary.

$\S$ Lawrence Livermore National Laboratory, Livermore, CA 94550.
}

\section{Work in Progress: Complex Substate Amplitudes Formed in Double Electron Capture (Publication 2)}

\section{H. Khemliche, M.H. Prior, and D. Schneider ${ }^{\dagger}$}

Electron capture is the dominant inelastic process in slow ( $\mathrm{V}<1 \mathrm{au}$ ) collisions of multiply charged ions with atoms, and, nearly always, the transferred electrons populate excited states of the product ion. In single electron capture the ion relaxes radiatively and the photon spectra, polarization, and/or angular distribution can be used to identify and characterize the excited state. This includes determination of the sense of rotation (orientation parameter) and angular properties of the charge density (e.g., alignment angle) of the captured electron. A similarly detailed description of the state formed in a multiple electron capture collision has been lacking, despite its expected bearing on, e.g., understanding the role of the interelectron interaction during the collision and the competition between pathways among the transient quasimolecular levels leading to the final state. This work describes the first such detailed study of a double electron capture collision. This was done on a system that is both theoretically and experimentally tractable: $\mathrm{C}^{5+}$ on $\mathrm{He}$. We have measured the scattering angle dependence of the complex amplitudes $\mathrm{a}_{\mathrm{M}}$ for populating the $\mathrm{IL}, \mathrm{M}>$ substates of the excited terms $1 s\left[2 s 2 p{ }^{1} P\right]{ }^{2} P$ and $1 s 2 p^{2}{ }^{2} D$ in $C^{3+}$ by transfer of both $\mathrm{He}$ electrons through analysis of anisotropy in the subsequent Auger electron emission from the decay of these states. This is analogous to studies of photon anisotropy in single electron capture studies; however, Auger decay is not bound by electric dipole selection rules and the final ion state is the $S$ state $\left(\mathrm{C}^{4+} 1 \mathrm{~s}^{2}\right)$ for all excited $\mathrm{C}^{3+} 1 \mathrm{~s} 212 \mathrm{l}^{\prime}$ terms, including ${ }^{2} \mathrm{D}$.

To determine the relative phases of the $\mathrm{a}_{M}$ the anisotropy must be measured with respect to the collision plane; this requires use of a coincidence technique. We measure the 1s2l21' Auger spectrum emitted into a small solid angle at polar angle $\theta_{e}$, with respect to the $\mathrm{C}^{5+}$ beam direction, in coincidence with two dimensional position detection of the scattered $\mathrm{C}^{4+}$ final state ions. A transformation of the coincident scattered projectile positions associated with each Auger line gives the azimuthal coordinate, $\phi_{\mathrm{e}}$, of the electron emission with respect to the scattering plane and, $\theta_{\mathrm{l}}$, the projectile ion 
scattering angle. A fit of the expressions for the anisotropy to the observations provides values for the complex substate amplitudes for populating the IL,M $>$ substates and their scattering angle (impact parameter) dependences. A previous noncoincidence study in this program provided values of the $\operatorname{la}_{\mathrm{M}} \mid$ averaged over all scattering angles by measuring the $\theta_{\mathrm{e}}$ dependence of the Auger emission rate integrated over all $\phi_{\mathrm{e}}$.

One use of the results of these measurements is to construct a picture showing how, for example, the $1 \mathrm{~s}(2 \mathrm{~s} 2 \mathrm{p}$ $\left.{ }^{1} \mathrm{P}\right){ }^{2} \mathrm{P}$ charge cloud varies with the scattering angle of the projectile. Figure 2-1 shows this for a collision energy of $25 \mathrm{keV}$. This series of pictures clearly demonstrates the effect of the loss of electron coupling to the rotating internuclear axis for increasingly close collisions (larger

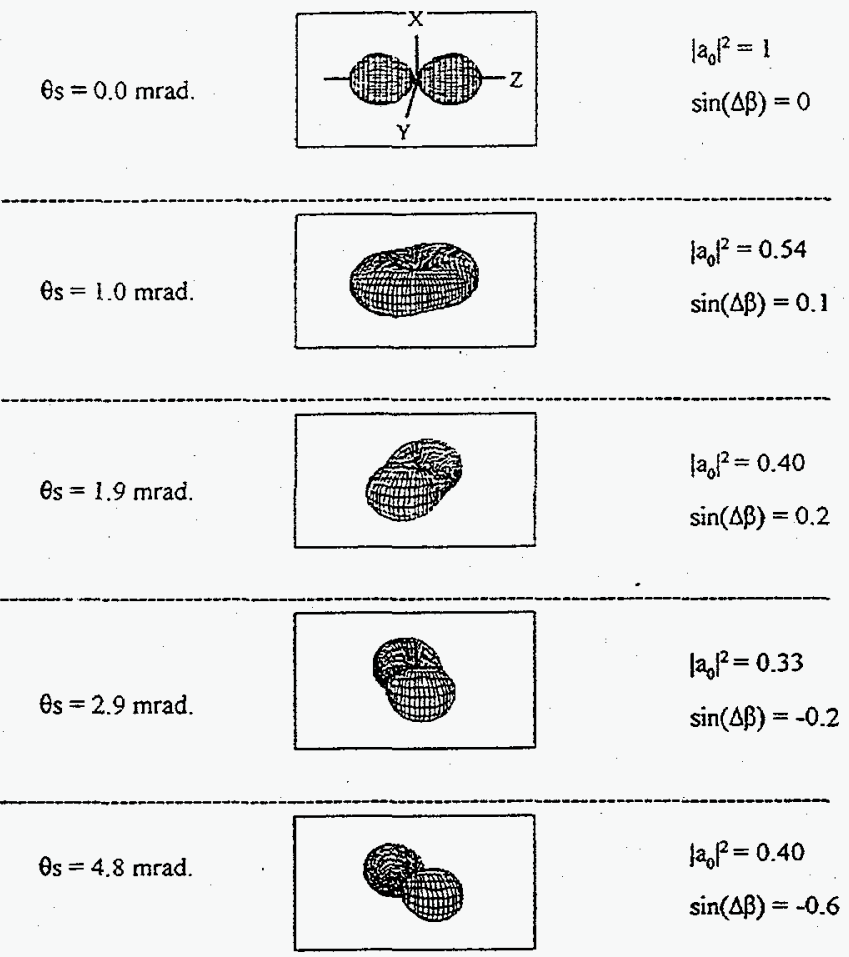

Figure 2-1. Variation of the $C^{3+} 1 s\left(2 s 2 p{ }^{1} P\right){ }^{2} P$ charge cloud with scattering angle for double capture by $\mathrm{C}^{5+}$ from He derived from measurements at $25 \mathrm{keV}$ collision energy. The initial relative velocity is along the $Z$ axis, the projectile scattering $\theta_{S}$, is in the $Y Z$ plane. The internuclear axis rotates 180 degrees during the collision, but as the scattering angle increases (closer collisions) the electron distribution is less able to follow the rotation and the cloud remains fixed at an intermediate orientation. la $\mathrm{l}$ is the magnitude of the complex amplitude for the $M=0$ substate and $\Delta \beta$ is the relative phase between the $M=0$ and $M=1$ amplitudes. scattering angles). The results also provide a measure of the component of angular momentum in the doubly excited state perpendicular to the scattering plane. The results are consistent with a propensity rule that favors population of substates with $\mathrm{M}=-\mathrm{L}$, for both the $1 \mathrm{~s}\left(2 \mathrm{~s} 2 \mathrm{p}{ }^{1} \mathrm{P}\right){ }^{2} \mathrm{P}$ and $1 \mathrm{~s} 2 \mathrm{p}^{2} \mathrm{D}$ levels.

The results of these measurements suggests that, for this collision system, the double capture is the outcome of nearly independent single electron transfers during the collision, with subsequent evolution into states more fully defined by the electron-electron interaction. Thus, for population of these equivalent electron configurations, this study shows no evidence of a correlated behavior during the transfer.

${ }_{\dagger}$ Lawrence Livermore National Laboratory.

3. Work in Progress: Production, Fragmentation and Lifetimes of Highly Charged Fullerene Ions

\section{J. Jian, M.H. Prior, and H. Khemliche}

The goal of this study is to determine the stability and decay modes of highly charged $C_{60}^{9+}$ ions made by the impact of slow, highly charged ions on a fullerene vapor target. It is extremely unusual for any molecule to remain intact carrying more than a few units of charge. However, previous studies have determined that $C_{60}^{q+}$ ions, with $q$ up to 7 , live at least a few microseconds, and as a preliminary to measuring their lifetimes, we have sought to determine whether ions with $\mathrm{q}>6$ can be produced by slow ion impact. Utilizing ion beams from the LBL Advanced Electron Cyclotron ion source, we have explored the production of the $\mathrm{C}_{60}^{\mathrm{q}+}$ ions for a wide range of projectile ions, i.e., ${ }^{40} \mathrm{Ar}^{4,8,12,16,17+},{ }^{136} \mathrm{X} \mathrm{e}^{27+}$, and ${ }^{209} \mathrm{Bi}^{38,44,46+}$ all with energies of $10 \mathrm{keV} / \mathrm{ch}$ arge. The $\mathrm{C}_{60}^{++}$ions are observed using a linear, second order space focused time-of-flight spectrometer, in delayed coincidence with electrons emitted from the collision. The observed ions have survived flight times of at least a few microseconds. The distribution of $\mathrm{C}_{60}^{\mathrm{q}+}$ ions shifts smoothly to higher $\mathrm{q}$ as the projectile charge increases. The mass-to-charge ratio spectrum of the slow collision products can be divided into two regions: (1) the fullerene ions and (2) a range of light fragments that appear at apparent multiples of $M=12$. Double hit spectra (first ion and second ion) show correlations consistent with pairs of singly charged ions generated from breakup of heavier doubly charged units. Figure 3-1 shows the time-of-flight spectrum showing the $C_{60,70}^{9 f}$ peaks obtained from ${ }^{136} \mathrm{Xe}^{27+}$ impact. 


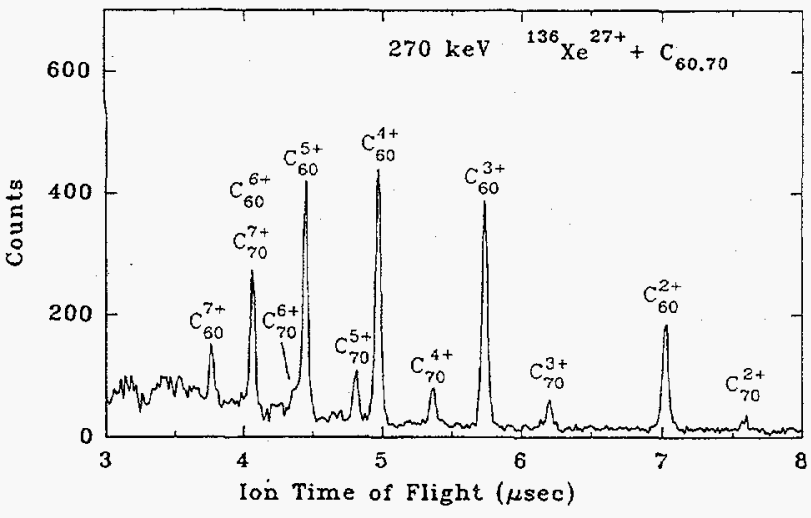

Figure 3-1. Time-of-flight spectrum showing $\mathrm{C}_{60,70}^{q+}$ ion peaks produced by impact of $270 \mathrm{keV}{ }^{136} \mathrm{Xe}^{27+}$ ions on fullerene vapor. The spectrum is a delayed coincidence between prompt electrons, and the arrival of slow heavy ions extracted from the collision region. The electrons result from a multiple electron transfer process; this precludes observation of $C_{60,70}^{+}$ ions that are produced by single electron transfer.

\section{Work in Progress: State Selective Charge}

Transfer-Absolute Cross Section Measurements for $\mathrm{O}^{6+}, \mathrm{Ne}^{8+}$ and $\mathrm{Mg}^{10+}$

\section{J.R. Crespo Lopez-Urrutia, ${ }^{\dagger \neq}$ E.E. Fill, ${ }^{\dagger}$ and M.H. Prior}

In this work, single electron charge exchange cross sections are measured for collisions of He-like oxygen, neon and magnesium ions at energies between 1 and $10 \mathrm{keV} / \mathrm{q}$ with neutral helium and argon gas targets. A reasonable knowledge of these cross sections is of interest for diagnostics of fusion plasmas as well as in astrophysics. More recently, growing interest in the possibility of a charge exchange-pumped $x$-ray laser has stimulated the search for reliable measurements of absolute cross sections in state-selective charge transfer processes.

The measurements are made using ion beams from the LBL Advanced Electron Cyclotron Resonance (AECR) Ion source. The ion beams are focused by an einzel lens into a differentially pumped gas cell where the collisions take place. Pressure in the gas cell is measured by a capacitance manometer. The ion beam is charge analyzed by electrostatic deflection following passage through the gas cell. Pressure studies of the growth of product ion beam intensities provide measurements of the absolute total electron capture cross sections for selected final projectile charge states.

State selective cross sections are obtained from VUV spectra of photons emitted from the gas cell. The line emission from the different $n$,I-states populated was resolved, providing information about how the total cross section is distributed among the states formed in the capture process. The instrument used is a $2.2 \mathrm{~mm}$ grazing incidence monochromator (McPherson 247) equipped with a $600 \mathrm{l} / \mathrm{mm}$ grating, and modified for computer control of the wavelength region scanned. The spectral region between 5.0 and $110 \mathrm{~nm}$ was covered with a resolution of $0.06 \mathrm{~nm}$ FWHM. The relative spectral sensitivity was determined using calculated branching ratios in Li-like and Na-like systems. A concave mirror in grazing incidence imaged the collision interaction region inside the gas cell onto the entrance slit of the monochromator. Line intensities are corrected for cascading contributions from higher lying states. The total experimental error is expected to be about $30 \%$ for the state selective cross sections. Good agreement has been obtained with results from other groups for the $\mathrm{O}^{6+}+\mathrm{He}$ system. Principal quantum numbers of the states populated are predicted reasonably well by the classical overbarrier model.

\footnotetext{
Max-Planck-Institut für Quantenoptic, Garching, Germany.

$\doteqdot$ Present address: Lawrence Livermore National Laboratory.
}

\section{FY 1994 PUBLICATIONS AND REPORTS}

\section{Refereed Journals}

1. V.L. Plano, R.R. Haar, J.A. Tanis, J. Palinkas, L. Sarkadi, P.A. Zavodszky, D. Berenyi, H. Khemliche, M.H. Prior, and D. Schneider, "Production of Low Energy Continuum Electrons at $0^{\circ}$ by $4.4 \mathrm{keV} / \mathrm{amu} \mathrm{O}^{8+}$ Ions," Nucl. Instrum. Meth. in Phys. Res. B86, 181 (1994).

\section{Other Publications}

2. H. Khemliche, M.H. Prior, and D. Schneider, "Complex Substate Amplitudes Formed in Double Electron Capture," Bull. Am. Phys. Soc. 39, 1215 (1994) and Abstracts of Contributed Papers, VII International Conference on the Physics of Highly Charged Ions, F. Aumayr and H.P. Winter, Editors (1994).

3. J. Jin, H. Khemliche, and M.H. Prior, "Production and Decay of Multiply Charged Fullerenes Created by Electron and Highly Charged Ion Impact," Bull. Am. Phys. Soc. 39, 1217 (1994).

4. H. Khemliche, "Determination Experimentale des Amplitudes de Population Complexes des Suos-Niveaux Magnetiques Issus d'une Double Capture Electronique a Bass Energie," Ph.D. Thesis, Universite Pierre et Marie Curie, Paris, France, September 30, 1994

\section{Invited Talks}

5. M.H. Prior, "Complex Substate Amplitudes Formed in Double Electron Capture," VII International Conference on the Physics of Highly Charged Ions, Technical University, Vienna, Austria, September 19-23, 1994. 


\section{PROCESSES AND TECHNIQUES}

\section{CHEMICAL ENERGY}

\section{High-Energy Oxidizers and Delocalized-Electron Solids*}

\author{
Neil Bartlett, Investigator
}

\section{INTRODUCTION}

The aim of this work is the synthesis and characterization of new two- and three-dimensional solids that may be useful in electrical energy storage. Fluorides are emphasized because fluorine is highly electronegative, small and lightweight. Thus high oxidation-state fluorides such as those of cobalt, nickel, copper, or silver have high oxidizing potential and low formula weights. Emphasis is placed on the thermodynamically unstable fluorides, which have sufficient kinetic stability to be easily stored. Such fluorides are not only powerful oxidizers, but the metal center in each is comparable in electronegativity to fluorine. It is probable, therefore, that some of the thermodynamically unstable fluorides will be metallic or even superconducting (like some copper oxide systems). Access to such fluorides is provided by salts of anions that are thermodynamically stable (e.g., $\mathrm{NiF}_{6}{ }^{2-}$ ). Kinetically stable, thermodynamically unstable fluorides, as reagents, can probably substitute for anodic oxidation processes (Simons process) for the preparation of fluorochemicals. Lithium cations in open-channel fluorides could provide ionic conductors stable to oxidation. New synthetic routes also provide access to ordered mixed rutile materials (e.g., $\mathrm{MnNiF}_{4}$ ). These should be ferrimagnets. Other "rutile" systems such as MNF (isoelectronic with $\mathrm{MO}_{2}$ ) like $\mathrm{CrO}_{2}$, could be metallic.

1. Synthesis of $\mathrm{CuNiF}_{6}$, a Powerful Oxidizer and Related $\mathrm{A}^{\mathrm{II}} \mathrm{Ni}^{\mathrm{IV}} \mathrm{F}_{6}$ Salts (Publication 4)

\section{Shen, S. Elder, and N. Bartlett}

The synthesis of nickel trifluoride from nickel(II) salts and $\mathrm{NiF}_{6}{ }^{2-}$ salts in anhydrous hydrogen fluoride (AHF) suggested that even more potent oxidizing fluorides than

*This work was supported by the Director, Office of Energy Research, Office of Basic Energy Sciences, Chemical Sciences Division, of the U.S. Department of Energy under Contract No. DE-AC08-76SF00098.
$\mathrm{NiF}_{3}$ might be prepared by substituting more oxidizing metal(II) species for the Ni(II). This has been achieved for $\mathrm{Cu}(\mathrm{II})$. As anticipated, the $\mathrm{NiF}_{6}{ }^{2-}$ salts of $\mathrm{Co}$ (II), and $\mathrm{Zn}$ (II) are not strong oxidizers and have structures closely related to $\mathrm{NiF}_{3}$. In all cases the magnetic data are in accord with the formulation $\mathrm{A}(\mathrm{II}) \mathrm{Ni}(\mathrm{IV}) \mathrm{F}_{6}$.

The $A(I I) N i(I V) F_{6}$ salts are prepared by adding an AHF solution of $\mathrm{A}^{\mathrm{II}}\left(\mathrm{MF}_{6}\right)_{2}$ salt to $\mathrm{K}_{2} \mathrm{NiF}_{6}$, also in $\mathrm{AHF}$, the desired salt being insoluble and the $\mathrm{KMF}_{6}$ salt sufficiently soluble in AHF to be removed by decantation and several washings by back distillation of AHF:

$$
\begin{aligned}
& \mathrm{A}\left(\mathrm{MF}_{6}\right)_{2}+\mathrm{K}_{2} \mathrm{NiF}_{6} \rightarrow \mathrm{ANiF}_{6} \downarrow+2 \mathrm{KMF}_{6} \\
& (\mathrm{~A}=\mathrm{Co}, \mathrm{Cu}, \mathrm{Zn} ; \mathrm{M}=\mathrm{As}, \mathrm{Sb}, \mathrm{Bi})
\end{aligned}
$$

$\mathrm{CuNiF}_{6}$ is a dark red-brown solid. Surprisingly it does not show any evidence of the Jahn-Teller distortion common to $\mathrm{Cu}(\mathrm{II})$ ( $d^{9}$ electron configuration) systems, but the rhombohedral unit cell (Table 1-1) is, as expected, slightly larger than that of $\mathrm{NI}(\mathrm{II}) \mathrm{Ni}(\mathrm{IV}) \mathrm{F}_{6}\left(\mathrm{NiF}_{3}\right)$. The obedience of the magnetic susceptibility of $\mathrm{CuNiF}_{6}$ to the Curie-Weiss law, and the absence of any field dependence, support the formulation $\mathrm{Cu}(\mathrm{II}) \mathrm{Ni}(\mathrm{IV}) \mathrm{F}_{6}$ \{ $\mathrm{Ni}(\mathrm{IV})$ being low spin $\left.\mathrm{d}^{6}\right\}$, although the value of the moment $(\mu=2.01$ B.M. $)$ is higher than the anticipated spin only moment of 1.73 B.M. Possibly, this is because the rhombohedral phase is a solid solution with some coprecipitated $\mathrm{NiF}_{3}$.

Solid $\mathrm{CuNiF}_{6}$ loses elemental $\mathrm{F}_{2}$ at about $40^{\circ} \mathrm{C}$ :

$$
\mathrm{CuNiF}_{6} \rightarrow \mathrm{CuNiF}_{4}+\mathrm{F}_{2}
$$

but does not decompose rapidly until $60^{\circ} \mathrm{C}$ and above. The solid product, $\mathrm{CuNiF}_{4}$, is a pseudo-rutile phase which is also observed whenever $\mathrm{CuNiF}_{6}$ acts as an oxidizer.

The room temperature synthesis of $\mathrm{CuNiF}_{6}$ in AHF leads to slight loss of fluorine (and corresponding

Table 1-1. The Unit Cell Parameters of $\mathrm{ANiF}_{6}(\mathrm{~A}=\mathrm{Co}$, $\mathrm{Ni}, \mathrm{Cu}, \mathrm{Zn}$ ).

\begin{tabular}{lcccc}
\hline $\mathrm{MNiF}_{6}$ & $\mathrm{a}(\AA)$ & $\lambda\left({ }^{\circ}\right)$ & $\mathrm{V}\left(\AA^{3}\right)$ & $\mathrm{V} / 2\left(\AA^{3}\right)$ \\
\hline $\mathrm{CoNiF}_{6}$ & 5.226 & 56.522 & 92.79 & 46.40 \\
$\mathrm{NiNiF}_{6}$ & 5.161 & 55.719 & 87.54 & 43.77 \\
$\mathrm{CuNiF}_{6}$ & 5.206 & 55.346 & 88.96 & 44.48 \\
$\mathrm{ZnNiF}_{6}$ & 5.218 & 55.427 & 89.79 & 44.89 \\
\hline
\end{tabular}


Table 1-2. Unit Cells of $\mathrm{CuNiF}_{4}, \mathrm{NiF}_{2}$ and $\mathrm{CuF}_{2}$.

\begin{tabular}{cll}
\hline \multicolumn{1}{c}{$\mathrm{CuNiF}_{4}$} & \multicolumn{1}{c}{$\mathrm{NiF}_{2}$} & \multicolumn{1}{c}{$\mathrm{CuF}_{2}$} \\
\hline $\begin{array}{l}\text { tetragonal } \\
\text { (Rutile type) }\end{array}$ & tetragonal & monoclinic \\
$\mathrm{a}=4.609 \AA$, & $\mathrm{a}=4.651 \AA$, & $\mathrm{a}=3.32 \AA$, \\
$\mathrm{c}=3.148 \AA$ & $\mathrm{c}=3.084 \AA$ & $\mathrm{b}=4.54 \AA$, \\
& & $\mathrm{c}=4.59 \AA$ \\
$\mathrm{V}=66.87 \AA^{3}$ & $\mathrm{~V}=66.69 \AA^{3}$ & $\mathrm{~V}=68.72 \AA^{3}$ \\
$\mathrm{Z}=1$ & $\mathrm{Z}=2$ & $\mathrm{Z}=2$ \\
\hline
\end{tabular}

contamination of the $\mathrm{CuNiF}_{6}$ ), but the dry solid can be stored (in FEP containers) for many weeks at $20^{\circ} \mathrm{C}$. In AHF suspension, however, the solid loses $\mathrm{F}_{2}$ at $20^{\circ} \mathrm{C}$ and is thereby fully reduced within 3 days.

$\mathrm{CuNiF}_{6}$ is reduced at $\sim 20^{\circ} \mathrm{C}$ with $\mathrm{Xe}$ (present in large molar excess):

$$
\mathrm{CuNiF}_{6}+\mathrm{Xe} \rightarrow \mathrm{CuNiF}_{4}+\mathrm{XeF}_{2}
$$

or with perfluoropropene, at $-20^{\circ} \mathrm{C}, \mathrm{C}_{3} \mathrm{~F}_{6}(\sim 100$ torr):

$$
\mathrm{CuNiF}_{6}+\mathrm{C}_{3} \mathrm{~F}_{6} \rightarrow \mathrm{CuNiF}_{4}+\mathrm{C}_{3} \mathrm{~F}_{8}
$$

If the partial pressure of $\mathrm{C}_{3} \mathrm{~F}_{6}$ in the last reaction approaches one atmosphere, the $\mathrm{CuNiF}_{6}$ becomes incandescent and $\mathrm{CF}_{4}$ with $\mathrm{C}_{2} \mathrm{~F}_{6}$ is then observed in the volatile products, the $\mathrm{CuNiF}_{4}$ product being much more crystalline.

There are slight variations in the $x$-ray powder patterns of the various $\mathrm{CuNiF}_{4}$ reaction products, but all are related to that of somewhat distorted $\mathrm{NiF}_{2}$ but much less distorted from tetragonal symmetry than is $\mathrm{CuF}_{2}$ (see Table 1-2).

Although the oxidizing properties of $\mathrm{Ni}(\mathrm{IV})$ allowed for the possibility of $\mathrm{CoNiF}_{6}$ being $\mathrm{Co}(\mathrm{III}) \mathrm{Ni}(\mathrm{III}) \mathrm{F}_{6}$, the simple paramagnetism and the magnetic moment, $\mu=$ 3.93 B.M., confirm that the appropriate formulation is Co(II) (high spin $d^{7}$ ) and Ni(IV) (low spin $d^{6}$ ) for which a spin only moment of $\mu=3.87$ B.M. is expected. Since $\mathrm{Co}$ (II) has the same $\mathrm{e}_{\mathrm{g}}{ }^{*}$ orbital population ( 2 electrons) as $\mathrm{Ni}^{\mathrm{II}}$ but a lower nuclear charge, the unit cell of $\mathrm{Co}(\mathrm{II}) \mathrm{Ni}(\mathrm{IV}) \mathrm{F}_{6}$ is larger than that of $\mathrm{Ni}(\mathrm{II}) \mathrm{Ni}(\mathrm{IV}) \mathrm{F}_{6}\left(\mathrm{NiF}_{3}\right)$ (Table 1-1) and $\mathrm{Co}(\mathrm{II}) \mathrm{Ni}(\mathrm{IV}) \mathrm{F}_{6}$ is an inferior oxidizer to $\mathrm{NiF}_{3}$.

2. Preparation and Structural and Magnetic Properties of $\mathrm{AgF}^{+} \mathrm{MF}_{6}-(\mathrm{M}=\mathrm{Ir}, \mathrm{Ru}, \mathrm{Sb}, \mathrm{Bi})$ and $\mathrm{Ag}^{2+}\left(\mathrm{BiF}_{6}\right)_{2}$ (Publication 5)

\section{G. Lucier, J. Münzenberg, W.J. Casteel, Jr., and N. Bartlett}

In earlier work it had been shown that $\mathrm{Ag}^{2+}$ in $\mathrm{AHF}$ was a sufficiently powerful electron oxidizer to oxidize xenon at $\sim 20^{\circ} \mathrm{C}$, or $\mathrm{O}_{2}$ (to $\mathrm{O}_{2}{ }^{+}$) at $-78^{\circ} \mathrm{C}$ and that it liberates $\mathrm{IrF}_{6}$ from $\mathrm{IrF}_{6}{ }^{-}$(ionization potential, $\mathrm{I}, \approx 7 \mathrm{eV}$ ). It had previously been observed by others ${ }^{1}$ that $\mathrm{Ag}^{2+}$ could be stabilized by $\mathrm{SbF}_{6}-$ in the solid state, as the salt $\mathrm{Ag}^{2+}\left(\mathrm{SbF}_{6}-\right)_{2}$, but that $\mathrm{AsF}_{6}-$ did not do so, and produced ${ }^{2}$ a one-dimensional fluorine-bridged cation, $\mathrm{AgF}^{\mathrm{n}+}{ }_{\mathrm{n}}$, in the salt $\mathrm{AgF}^{+} \mathrm{AsF}_{6}$-. Work in these laboratories had already shown that this cation, although less oxidizing than $\mathrm{Ag}^{2+}$, did oxidize $\mathrm{OsF}_{6}-(\mathrm{I} \approx 6 \mathrm{eV})$ to $\mathrm{OsF}_{6}$, but the limitations to the existence of $\mathrm{AgF}^{+}$or $\mathrm{Ag}^{2+}$ salts had not been defined.

It has been found that $\mathrm{AgFMF}_{6}$ salts can be conveniently made by treatment of $\mathrm{AgMF}_{6}$ relatives, in suspension in liquid anhydrous hydrogen fluoride (AHF), with elemental fluorine at $\sim 20^{\circ} \mathrm{C}$. The slight solubility of the $\mathrm{AgMF}_{6}$ salts, and the even lower solubility of the $\mathrm{AgFMF}_{6}$ products, favor the formation of sufficiently large crystals for structural analysis by $\mathrm{x}$-ray diffraction, as well as other physical characterization. The new salts $\mathrm{AgF}^{+} \mathrm{MF}_{6}^{-}(\mathrm{M}=\mathrm{Ir}, \mathrm{Ru}, \mathrm{Sb}, \mathrm{Bi})$ have been prepared in this way. Attempts to prepare $\mathrm{AgF}^{+} \mathrm{PF}_{6}-$ led to precipitation of $\mathrm{AgF}_{2}$ and release of $\mathrm{PF}_{5}$ :

$$
\mathrm{AgPF}_{6}+1 / 2 \mathrm{~F}_{2} \rightarrow \mathrm{AgF}_{2}+\mathrm{PF}_{5}
$$

This contrasts with the successful synthesis of $\mathrm{AgFBF}_{4}$ in these laboratories. Although the gaseous fluoride-ion affinity of $\mathrm{BF}_{3},\left\{-\Delta \mathrm{H}^{\circ}\left(\mathrm{BF}_{3(\mathrm{~g})}+\mathrm{F}_{(\mathrm{g})}{ }^{-} \rightarrow \mathrm{BF}_{4(\mathrm{~g})}\right)^{-}\right)=92 \mathrm{kcal}$ $\mathrm{mol}^{-1}$ \} is about $9 \mathrm{kcal} \mathrm{mol}^{-1}$ smaller than that for $\mathrm{PF}_{5}$, the lattice enthalpy of $\mathrm{AgFBF}_{4}$ must be greater than that of $\mathrm{AgFPF}_{6}$ because the $\mathrm{BF}_{4}{ }^{-}$is smaller than $\mathrm{PF}_{6}{ }^{-}$(by the volume of two $\mathrm{F}$ ligands). For smaller-cation salts, this has been previously shown ${ }^{3}$ to give $5 \mathrm{kcal} \mathrm{mol}^{-1}$ advantage per $F$ ligand to the smaller anion salt (i.e., $\sim 10 \mathrm{kcal} \mathrm{mol}^{-1}$ here). Thus, the failure to prepare $\mathrm{AgF}^{+} \mathrm{PF}_{6}^{-}$points to borderline stability of $\mathrm{AgF}^{+} \mathrm{BF}^{-}{ }_{4}$ which is consistent with the ready dissociation of the latter salt to $\mathrm{AgF}_{2}$ and $\mathrm{BF}_{3}$ in the $\mathrm{AHF}$ medium. It can be reliably concluded that superior $\mathrm{F}^{-}$ acceptors to $\mathrm{PF}_{5}$ are needed to stabilize $(\mathrm{AgF})^{\mathrm{n}+}{ }_{\mathrm{n}}$ salts. The anions $\mathrm{MF}_{6}{ }^{-}(\mathrm{M}=\mathrm{Ru}, \mathrm{Rh}, \mathrm{Ir}, \mathrm{Pt}$ and $\mathrm{Au})$ have high enough ionization potentials $(>7 \mathrm{eV})$ to resist transfer of an electron from the anion to $(\mathrm{AgF})^{\mathrm{n}+}{ }_{\mathrm{n}}$, but in none of these cases has a $\mathrm{Ag}^{2+}\left(\mathrm{MF}_{6}{ }^{-}\right)_{2}$ salt been prepared. The novel bismuth compound is isostructural with its antimony relative, and the structure is illustrated in Figure 2-1.

Both the structure and the magnetic properties of $\mathrm{Ag}\left(\mathrm{BiF}_{6}\right)_{2}$ are consistent with its being an $\mathrm{Ag}^{2+}$ salt. The magnetic susceptibility temperature dependence is obedient to the Curie-Weiss law, as shown in Figure 2-2, although the moment $(\mu=2.1$ B.M. $)$ is slightly larger than that expected for a $d^{9}$ configuration ion. As may be seen from Figure 2-1, each $\mathrm{Ag}^{2+}$ ion is closer to four surrounding $\mathrm{F}$ ligands of four separate $\mathrm{BiF}_{6}\{2$ at $2.096(9)$ and 2 at $2.122(9) \AA$ \} in roughly square array about the cation. There are two other more distant $F$ ligands of two separate anions, 


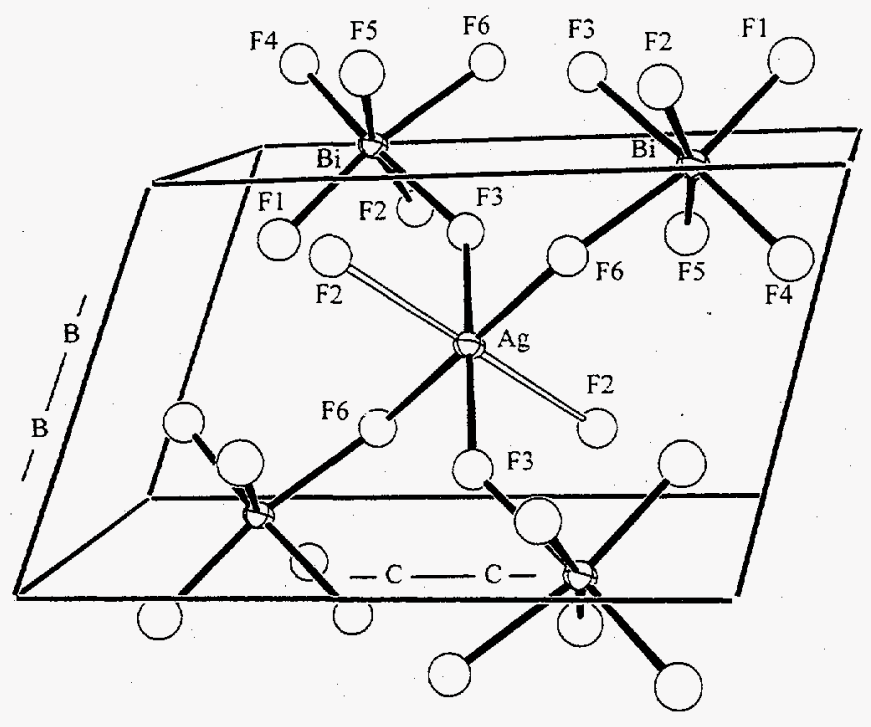

Figure 2-1. ORTEP of $\mathrm{Ag}\left(\mathrm{BiF}_{6}\right)_{2}$.

nearly perpendicular to the nearly square $\mathrm{AgF}_{4}$ arrangement, with $\mathrm{Ag} \cdots \mathrm{F}=2.44(1) \AA$. The filled $d_{\mathrm{z}} 2$ orbital of the $\mathrm{Ag}(\mathrm{II}) d^{9}$ configuration accounts for this elongation ( $\mathrm{z}$ axis) of the $\mathrm{Ag}(\mathrm{II})$ octahedral $\mathrm{F}$ ligand environment. The high effective nuclear charge of the $\mathrm{Ag}(\mathrm{II})$, which is responsible for the remarkable oxidizing properties of these compounds, is manifested in the xy plane. The $\mathrm{F}$ ligands that make close approach to the $\mathrm{Ag}$ (II) in that plane are pulled from the anion, resulting in appreciable distortions from octahedral symmetry of the anion.

The preparation of $\mathrm{AgFMF}_{6}(\mathrm{M}=\mathrm{Sb}$ or $\mathrm{Bi})$ shows these salts are thermodynamically stable with respect to $\mathrm{AgF}_{2}$ (which is of low solubility in $\mathrm{AHF}$ ) and $\mathrm{Ag}\left(\mathrm{MF}_{6}\right)_{2}$ (each soluble in AHF). X-ray powder diffraction data show that the two $\mathrm{AgFMF}_{6}(\mathrm{M}=\mathrm{Sb}$ or $\mathrm{Bi})$ salts are structurally close, but of different structure from either $\mathrm{AgFMF}_{6}(\mathrm{M}=$ As, Ir or $\mathrm{Au}$ ) or $\mathrm{AgFRuF}_{6}$. All of the known $\mathrm{AgFMF}_{6}$ salts are characterized by a temperature-independent paramagnetism similar to that observed in one-dimensional metals. This is illustrated by the data for $\mathrm{AgFBiF}_{6}$ shown in Figure 2-2, where the contrast to that of $\mathrm{Ag}\left(\mathrm{BiF}_{6}\right)_{2}$ is brought out. Evidently, there is a strong coupling of the $\operatorname{Ag}(\mathrm{II}) d^{9}$ moments, as a consequence of interaction through bridging $\mathrm{F}$ ligands. Bridging $\mathrm{F}$ ligands for $\mathrm{Ag}$ (II) occur in both $\mathrm{AgFIrF}_{6}$ (Figure 2-3) and $\mathrm{AgFRuF}_{6}$ (Figure 2-4) and are assumed to be present in $\mathrm{AgFBi}(\mathrm{Sb}) \mathrm{F}_{6}$ also. All attempts to detect metallic type conductivity in single crystals of the various $\mathrm{AgFMF}_{6}$ salts, by applying gold electrodes, have been unsuccessful. It therefore appears that the $(\mathrm{AgF})^{\mathrm{n}+}{ }_{\mathrm{n}}$ cations have a sufficiently large band gap to be effective insulators. (Since $\mathrm{Ag}(\mathrm{II})$ approaches $\mathrm{F}$
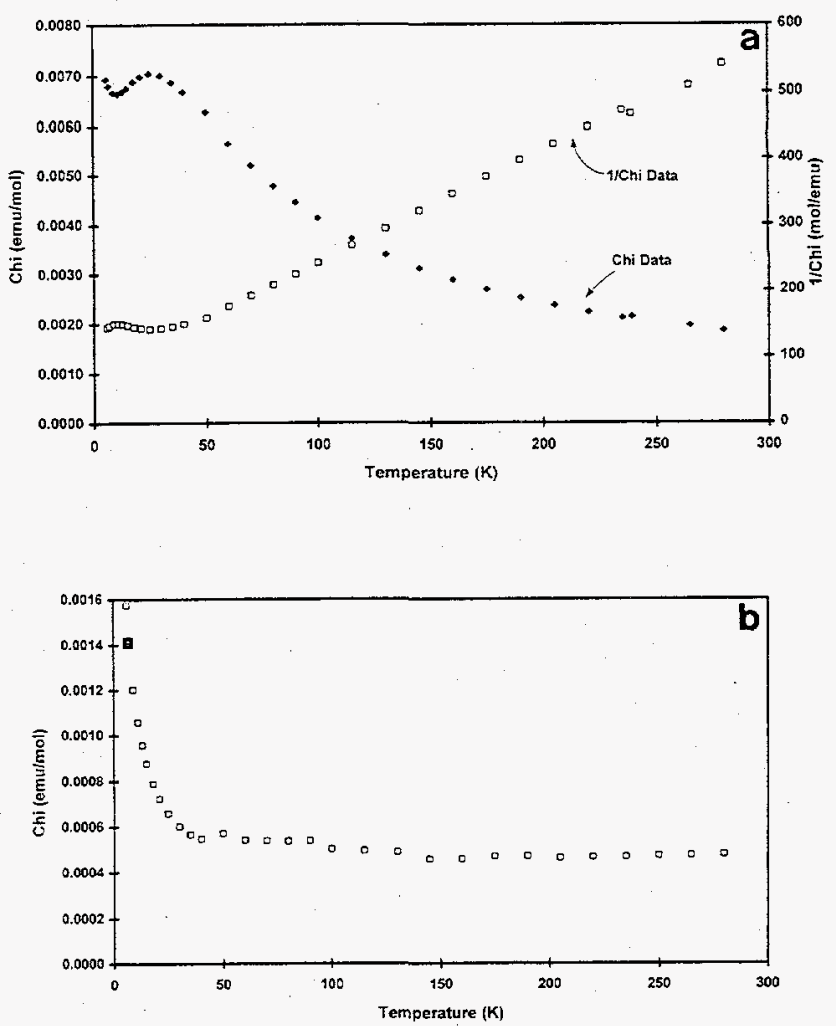

Figure 2-2. (a) Magnetic susceptibility data of $A g\left(\mathrm{BiF}_{6}\right)_{2}$ in a $5 \mathrm{kG}$ field. Simple paramagnetism is observed above $40 \mathrm{~K}, \mu_{\text {eff }}=2.1$ B.M. (b) Magnetic susceptibility of $\mathrm{AgFBiF}_{6}$ in a $5 \mathrm{kG}$ field showing a trend of temperature-independence from $40 \mathrm{~K}$ up to $280 \mathrm{~K}$. The data are also fieldindependent.

ligand in electronegativity in these compounds, metallic conductivity may yet be found).

The close structural similarity of most $\mathrm{IrF}_{6}^{-}$and $\mathrm{RuF}_{6}-$ salts is illustrated by the $\mathrm{LiMF}_{6}$ salts where the $\mathrm{RuF}_{6}{ }^{-}$ formula unit volume is only $0.3 \AA^{3}$ smaller. The marked

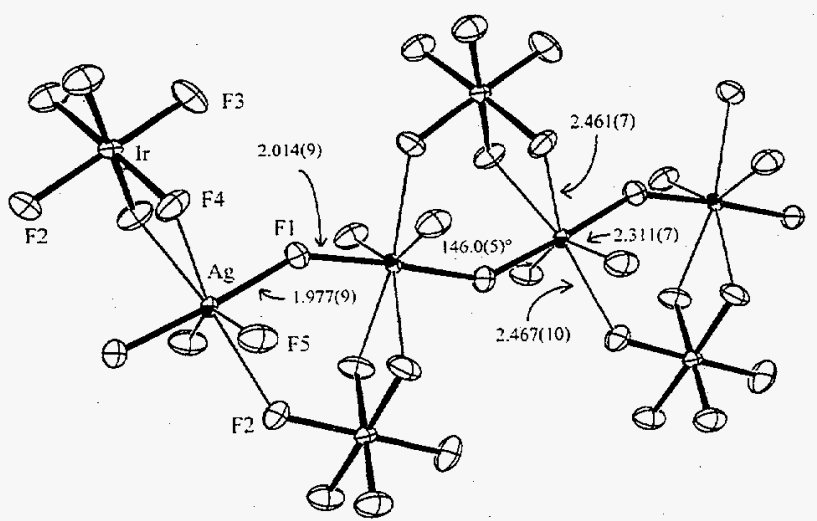

Figure 2-3. ORTEP of $\mathrm{AgFIrF}_{6}$. The $\mathrm{Ag}(\mathrm{II})$ is linearly coordinated and linked through $F 1$ in a one-dimensional chain cation (distances in $\AA$ ). 


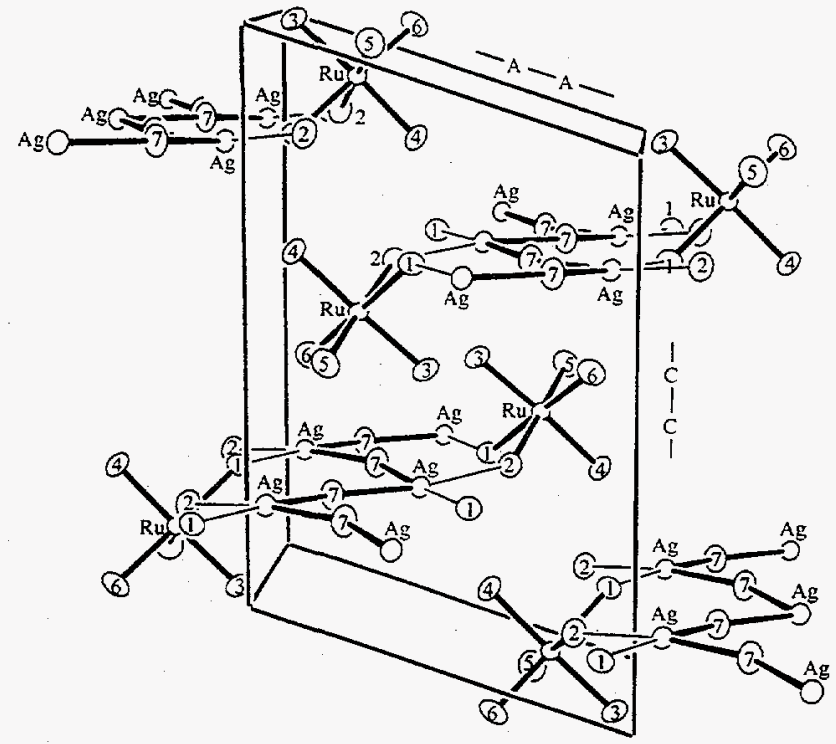

Figure 2-4. ORTEP of $\mathrm{AgFRuF}_{6}$. The $\mathrm{Ag}(\mathrm{II})$ is square coordinated and linked through $\mathrm{F} 7$ in a one-dimensional chain cation.

difference in structure of the $\mathrm{AgF}^{+}$salts was therefore not expected. Since $\mathrm{AgFIrF}_{6}$ (Figure 2-3) has the same structure as $\mathrm{AgFAsF}_{6}$, yet the $\mathrm{IrF}_{6}{ }^{-}$anion is larger by $7.5 \AA^{3}$, it is evident that the linearly coordinated $\mathrm{Ag}$ (II) adapts to the differing anions sizes by a change in the bridging angle $\mathrm{Ag}-\mathrm{F}-\mathrm{Ag}$. The reason for the new structure, represented by $\mathrm{AgFRuF}_{6}$ (Figure 2-4) is therefore unlikely to be connected to anion size. In $\mathrm{AgFRuF}_{6}$, the $\mathrm{Ag}$ (II) is roughly square-coordinated by $\mathrm{F}$ ligands, as in $\mathrm{AgF}_{2}$. This is probably a consequence of the $\mathrm{F}$ ligand having, in $\mathrm{RuF}_{6}$, a lower negative charge than in the $\operatorname{IrF}_{6}{ }^{-}$case (N.B. the electron affinity of $\mathrm{RuF}_{6}$ is $1 \mathrm{eV}$ higher than that of $\operatorname{IrF}_{6}$ ). As anticipated by Crystal Field Theory for a $d^{9}$ configuration, the approximately square arrangement of the $\mathrm{Ag}(\mathrm{II})$ in the $\mathrm{AgFRuF}_{6}$ structure is the alternative to the linear coordination seen in $\mathrm{AgFIrF}_{6}$.

1. D. Gantar, I. Leban, and B. Frlec, J. Chem. Soc. Dalton Trans., 2379 (1987).

2. D. Gantar, B. Frlec, D.R. Russell, and J.H. Holloway, Acta Cryst. C43, 618 (1987)

3. T. E. Mallouk, G.L. Rosenthal, G. Müller, R. Brusaco, and N. Bartlett, Inorg. Chem. 23, 3167 (1984).

\section{The O-O Distance in $\mathrm{O}_{2}{ }^{+}$from the Crystal Structure of $\mathrm{O}_{2} \mathrm{RuF}_{6}$ (Publication 6)}

\section{P. Botkovitz, F. Hollander, and N. Bartlett}

A neutron diffraction study by Ibers and Hamilton ${ }^{1}$ of $\mathrm{O}_{2} \mathrm{PtF}_{6}$ established the presence of discrete $\mathrm{O}_{2}$ and $\mathrm{PtF}_{6}$ species in the cubic crystal lattice but did not provide a definitive value for the $\mathrm{O}-\mathrm{O}$ bond length that has remained experimentally undetermined for the cation $\mathrm{O}_{2}{ }^{+}$. Recent work in these laboratories had shown that $\mathrm{O}_{2}{ }^{+}\left(\mathrm{MF}_{6}\right)^{-}$salts could be recovered in nearly $100 \%$ yield from AHF, and this encouraged a quest for single crystals. A small, deepred (nearly black) crystal of $\mathrm{O}_{2}+\mathrm{RuF}_{6}{ }^{-}$[the Raman spectrum of which showed $\lambda\left(\mathrm{O}_{2}^{+}\right)=1837 \mathrm{~cm}^{-1}$ in agreement with J.E. Griffiths et al. ${ }^{2}$ ] provided 1559 raw intensity data at $146 \mathrm{~K}$.

The unit cell was shown to be cubic, $a=9.9126(15) \AA$ at $146 \mathrm{~K}$, space group Ia3. The data were corrected for decay and absorption effects and, after averaging of equivalent data, removal of systematically absent data left 241 unique data in the final set. The structure determination was started with substitution of the $\mathrm{Pt}$ and $\mathrm{F}$ position of the $\mathrm{O}_{2} \mathrm{PtF}_{6}$ structure, ${ }^{1}$ by $\mathrm{Ru}$ and $\mathrm{F}$, with refinement using standard least-squares and Fourier techniques. Inspection of the Fourier and difference Fourier maps, following anisotropic refinement of the $F$ atom, showed clearly that the electron density for the oxygen atoms was consistent with offset of the oxygen atoms from the threefold axis. The oxygen atom was constrained to have an anisotropic thermal ellipsoid parallel to the threefold axis.

Figure 3-1 shows the difference Fourier that demonstrates the threefold disorder of the $\mathrm{O}_{2}{ }^{+}$, which is

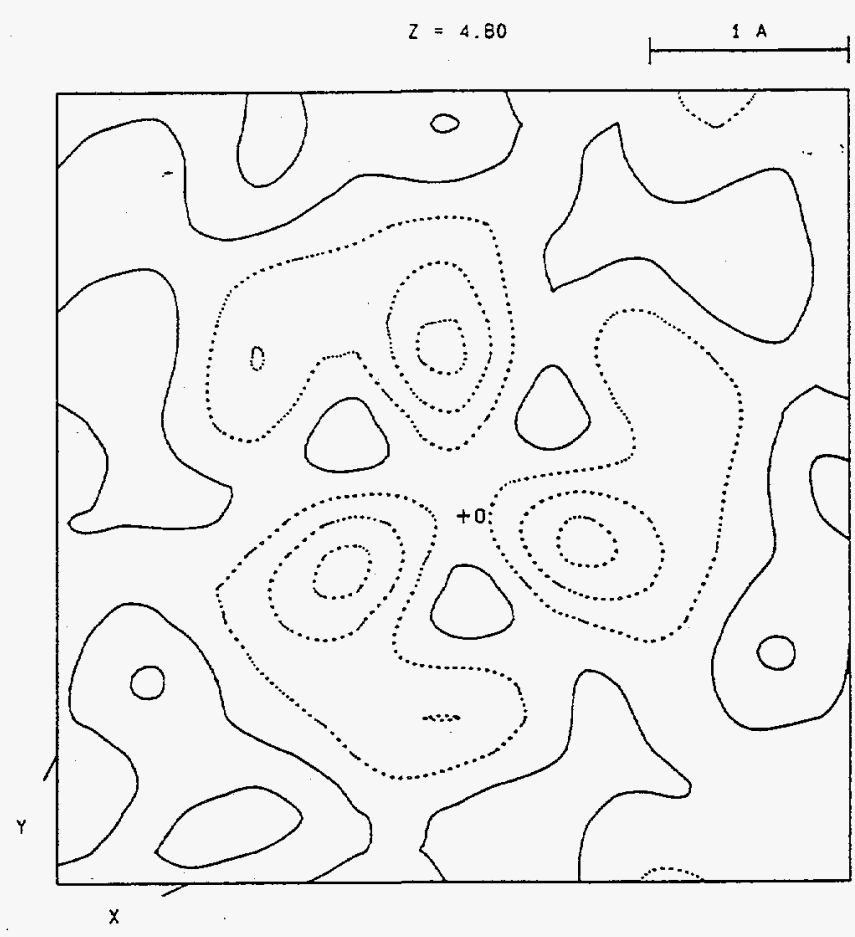

Figure 3-1. Difference Fourier showing the threefold disorder of $\mathrm{O}_{2}{ }^{+}$in $\mathrm{O}_{2} \mathrm{RuF}_{6}$. 
tilted with respect to the threefold axis. The final residuals for 16 variables refined against the 130 data for which $F^{2}>3 \sigma\left(F^{2}\right)$ were $R=1.72 \%$, wR $=2.17 \%$ and $\mathrm{GOF}=$ 0.865 . For all 241 data $\mathrm{R}$ was $3.93 \%$. The $\mathrm{O}-\mathrm{O}$ distance in $\mathrm{O}_{2}{ }^{+}$was found to be $1.125(17) \AA$, which is to be compared with the distance of $1.123 \AA$ derived for the gaseous $\mathrm{O}_{2}{ }^{+}$ species reported ${ }^{3}$ by Herzberg. The Ru-F distance in the nearly octahedral anion is $1.852(4) \AA$ at $146 \mathrm{~K}$.

1. J.A. Ibers and W.C. Hamilton, J. Chem. Phys. 44, 1748 (1966).

2. J.E. Griffiths, W.A. Sunder, and W.E. Falconer, Spectrochimica Acta 31A, 1207 (1975).

3. G. Herzberg, "The Spectra of Diatomic Molecules," D. Van Nostrand Company, Inc., Princeton, NJ, 1950, p. 560.

\section{FY 1994 PUBLICATIONS AND REPORTS}

\section{Refereed Journals}

I. C, Shen, R. Hagiwara, T.E. Mallouk, and N. Bartlett, "Thermodynamic Aspects of the Remarkable Oxidizing Capabilities of Fluorine-Lewis-Fluoroacid Mixtures," ACS Symposium Series No. 555, Inorg. Fluorine Chem., 26 (1994).

\section{LBL Reports}

2. R. Hagiwara and N. Bartlett, "Some Thermodynamic Aspects of the Intercalation of Graphite by Fluoroanions" in Fluorine-Carbon and Fluoride-Carbon Materials, $\mathrm{T}$. Nakajima, ed. (Marcel Dekker, Inc., New York, 1994), Chap. 3; LBL-36260.

3. M. Kawaguchi and N. Bartlett, "Synthesis, Structure, and Intercalation Chemistry of $\mathrm{B} / \mathrm{C} / \mathrm{N}$ Materials Based on the Graphite Network" in Fluorine-Carbon and FluorideCarbon Materials, T. Nakajima, ed. (Marcel-Dekker, Inc., New York, 1994), Chap. 5; LBL-36259.

4. C. Shen, S. Elder, and N. Bartlett, "Synthesis of $\mathrm{CuNiF}_{6}$, a Powerful Oxidizer, and Related $\mathrm{A}^{\mathrm{II}} \mathrm{Ni}^{\mathrm{IV}} \mathrm{F}_{6}$, Salts," LBL36715.

5. G. Lucier, J. Münzenberg, W.J. Casteel, Jr., and N. Bartlett, "Preparation and Structural and Magnetic Properties of
$\mathrm{AgF}^{+} \mathrm{MF}_{6}^{-}(\mathrm{M}=\mathrm{Ir}, \mathrm{Ru}, \mathrm{Sb}, \mathrm{Bi})$ and $\mathrm{Ag}^{2+}\left(\mathrm{BiF}_{6}{ }^{-}\right)_{2}$," Inorg. Chem. (in press); LBL-36089.

6. P. Botkowitz, F. Hollander, and N. Bartlett, "The Interatomic Distance in $\mathrm{O}_{2}{ }^{+}$in $\mathrm{O}_{2} \mathrm{RuF}_{6}$, , LBL-36705.

\section{Invited Talks}

7. N. Bartlett, "Oxygen as a Reducing Agent: Some Chemistry of High Oxidation-State Cations in Liquid HF," Oregon State University, Corvallis, OR, November 1, 1993.

8. N. Bartlett, "Thermodynamically Unstable Transition Element-Fluorides and Their Remarkable Oxidizing Properties," Rutgers University, New Brunswick, NJ, April 22, 1994

9. N. Bartlett, "The Oxidizing Properties of Cationic HighOxidation State Transition Element Fluoro Species," 20th American Chemical Society National Meeting, San Diego, CA, March 14, 1994.

10. N. Bartlett, "Thermodynamically Unstable Transition Element Fluorides and Their Remarkable Oxidizing Properties," George H. Cady Lecture, University of Washington, Seattle, WA, May 24, 1994.

11. N. Bartlett, "History of the Discovery of Argon and Its Implications for Chemistry," Eighth International Conference on Geochronology, Cosmochronology, and Isotope Geology, Berkeley, CA, June 6, 1994.

12. N. Bartlett, "Some Chemistry of High Oxidation State Transition Metal Fluorides in Anhydrous HF," International Conference on Fluorine Chemistry, Kyoto, Japan, July 1994.

13. N. Bartlett, "Interrelationships of $\mathrm{Ag}(\mathrm{II}), \mathrm{Ag}(\mathrm{I})$, and $\mathrm{Ag}(\mathrm{III})$ in Liquid Anhydrous Hydrogen Fluoride Solution," 14th International Symposium on Fluorine Chemistry, Yokohama, Japan, August 1-5, 1994.

14. N. Bartlett, "Thermodynamically Unstable Fluorides," Freie Universität Berlin, Germany, September 9, 1994; Universität Gottinger, September 12; and Universität Bremen, Germany, September 13, 1994.

15. N. Bartlett, "Thermodynamically Unstable Transition Element Fluorides and Their Remarkable Oxidizing Properties," Durham University, England, October 13, 1994.

16. N. Bartlett, "Some Chemistry of Ag I, II and III in Anhydrous Hydrogen Fluoride Solution," Durham University, England, October 19, 1994. 


\section{Catalytic Conversion of $\mathbf{C}_{\mathbf{1}}$ Compounds*}

Alexis T. Bell, Investigator

\section{INTRODUCTION}

The purpose of this program is to develop an understanding of the fundamental processes involved in the catalytic conversion of $\mathrm{C}_{1}$ compounds, such as $\mathrm{CO}, \mathrm{CO}_{2}$, and $\mathrm{CH}_{4}$, to fuels and chemicals. Attention is focused on defining the factors that limit catalyst activity, selectivity, and resistance to poisoning, and the relationship between catalyst composition/structure and performance. To meet these objectives, a variety of surface diagnostic techniques are used to characterized supported and unsupported catalysts before, during, and after reaction. The information thus obtained is combined with detailed studies of reaction kinetics to elucidate reaction mechanisms and the influence of modifications in catalyst composition and/or structure on the elementary reactions involved.

\section{An Infrared Study of Methanol Synthesis from} $\mathrm{CO}_{2}$ on Clean and Potassium-Promoted $\mathrm{Cu} / \mathrm{SiO}_{2}$ (Publication 10)

\section{D.B. Clarke, D.-K. Lee, M.J. Sandoval, and A.T. Bell}

Infrared spectroscopy and temperature-programmed reaction (TPR) spectroscopy were used to study the hydrogenation of $\mathrm{CO}_{2}$ and $\mathrm{CO}_{2} / \mathrm{CO}$ mixtures over $\mathrm{Cu} / \mathrm{SiO}_{2}$ and potassium-promoted $\mathrm{Cu} / \mathrm{SiO}_{2}$ catalysts. Isothermal and temperature-programmed reactions were conducted at temperatures between $303-563 \mathrm{~K}$ and pressures of 0.1 and $0.72 \mathrm{MPa}$. The only products observed during the reaction of $\mathrm{H}_{2}$ and $\mathrm{CO}_{2}$ are $\mathrm{CO}, \mathrm{H}_{2} \mathrm{O}$, and $\mathrm{CH}_{3} \mathrm{OH}$. At $0.1 \mathrm{MPa}$ over $\mathrm{Cu} / \mathrm{SiO}_{2}$, only $1 \%$ of the $\mathrm{CO}_{2}$ reacts to form methanol, the balance forming $\mathrm{CO}$ via the reverse water-gas-shift (RWGS) reaction. Increasing the total pressure to $0.72 \mathrm{MPa}$ and addition of $\mathrm{CO}$ to the feed $\left(\mathrm{CO} / \mathrm{CO}_{2}=1\right)$ improve the methanol selectivity to $26 \%$. Apparent activation energies measured at $480 \mathrm{~K}$ during $\mathrm{H}_{2} / \mathrm{CO}_{2} / \mathrm{Ar}$ reaction at $0.72 \mathrm{MPa}$ are $21.1 \mathrm{kcal} / \mathrm{mol}$ for methanol synthesis and $15.2 \mathrm{kcal} / \mathrm{mol}$ for the RWGS reaction. Substitution of $\mathrm{CO}$ for $\mathrm{Ar}$ in the feed does not affect the apparent activation barrier for RWGS but lowers that for

*This work was supported by the Director, Office of Energy Research, Office of Basic Energy Sciences, Chemical Sciences Division, of the U.S. Department of Energy under Contract No. DE-AC03-76SFO0098. methanol synthesis to $16.9 \mathrm{kcal} / \mathrm{mol}$. Infrared spectra reveal the following species on the $\mathrm{Cu}$ surface during $\mathrm{CO}_{2}$ hydrogenation: bidentate formate, monodentate formate, $\mathrm{H}_{2} \mathrm{O}, \mathrm{CO}$, and two forms of carbonate. Small concentrations of methanol and methoxy species are also observed. Addition of $\mathrm{CO}$ to the $\mathrm{H}_{2} / \mathrm{CO}_{2}$ feed increases both the bidentate coverage and the turnover frequency for methanol synthesis by $40 \%$. Potassium promotion of $\mathrm{Cu} / \mathrm{SiO}_{2}$ accelerates the RWGS reaction but hinders methanol synthesis, reducing the methanol synthesis selectivity to $1 \%$ at $0.72 \mathrm{MPa}$ during the reaction of $\mathrm{H}_{2}$ and $\mathrm{CO}_{2}$. Infrared observations show that potassium promotion stabilizes formate and carboxylate species. Based on the species observed during TPR-IR experiments and previous insights from methanol decomposition studies, a mechanism is proposed for $\mathrm{CO}_{2}$ hydrogenation over $\mathrm{Cu}$. Figure 1-1 illustrates the pathways involved in methanol synthesis and the RWGS reaction.

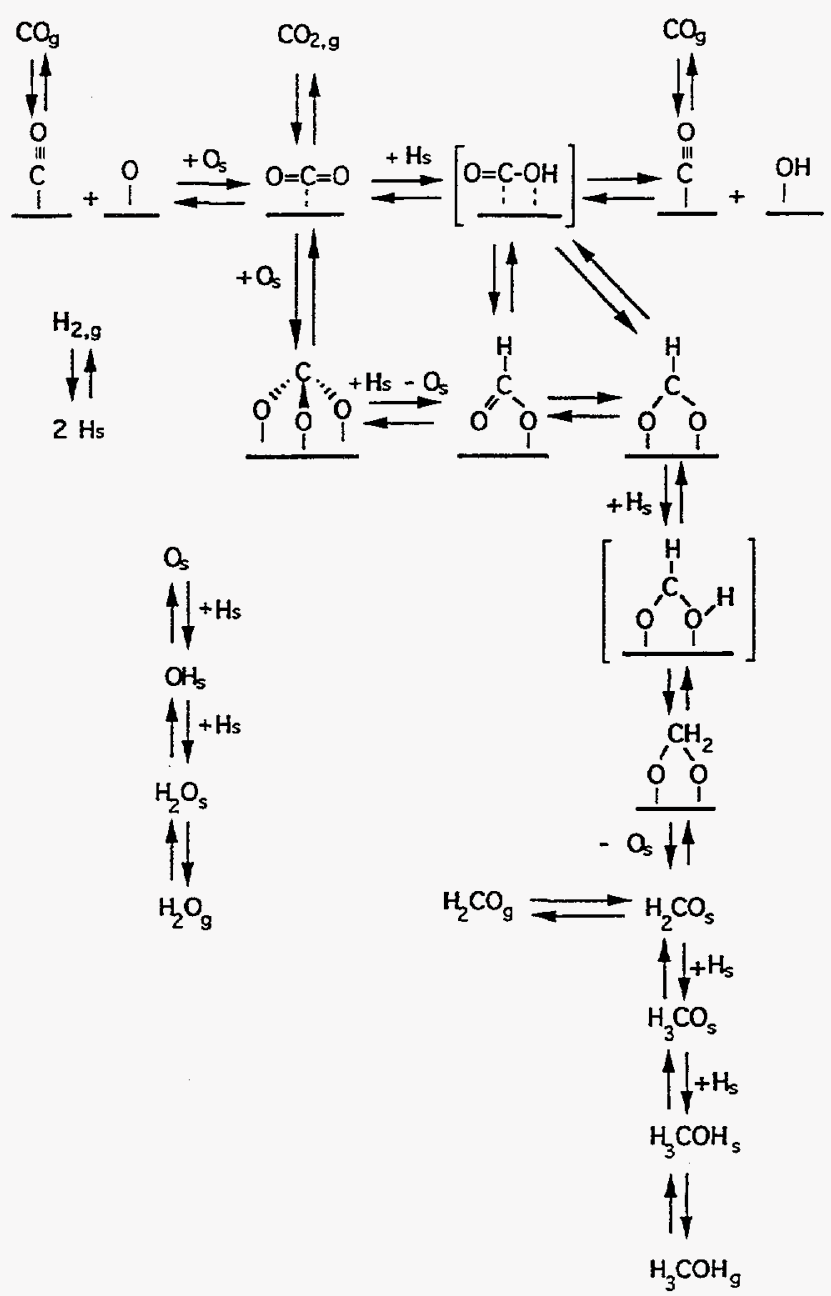

Figure 1-1. Reaction mechanisms for methanol synthesis and the reverse water-gas-shift reaction over copper. 
2. Effects of Dispersion and Metal-Metal Oxide Interactions on Fischer-Tropsch Synthesis over $\mathrm{Ru} / \mathrm{TiO}_{2}$ and $\mathrm{TiO}_{2}-$ Promoted $\mathrm{Ru} / \mathrm{SiO}_{2}$ (Publication 6)

\section{T. Komaya, A.T. Bell, Z. Weng-Sieh, R. Gronsky, F. Engleke, T.S. King, and M. Pruski}

The activity of $\mathrm{Ru} / \mathrm{TiO}_{2}$ and $\mathrm{TiO}_{2}$-promoted $\mathrm{Ru} / \mathrm{SiO}_{2}$ catalysts for Fischer-Tropsch synthesis has been investigated. The Ru dispersion of these catalysts was determined by TEM, and the fraction of the Ru surface available for $\mathrm{H}_{2}$ adsorption was determined by ${ }^{1} \mathrm{H}$ NMR. Even after low-temperature reduction, the surface of titania containing catalysts is covered by titania to a substantial degree. The specific activity and selectivity of these catalysts are dominated by the interactions occurring between $\mathrm{Ru}$ particles and the titania overlayer. As the fraction of the $\mathrm{Ru}$ particle surface covered by titania increases, the turnover frequency for $\mathrm{CO}$ consumption passes through a maximum, while that for methane formation decreases monotonically. The probability for chain growth and the olefin-to-paraffin ratio of the products increase with increasing titania coverage. These trends are attributed to the effects of the titania overlayer on the catalytic properties of $\mathrm{Ru}$.

\section{Effects of Sodium on the Structure and Fischer-} Tropsch Synthesis Activity of $\mathrm{Ru} / \mathrm{TiO}_{2}$ (Publication 9)

\section{T. Komaya, A.T. Bell, Z. Weng-Sieh, R. Gronsky, F. Engleke, T.S. King, and M. Pruski}

The influence of sodium on the migration of titanium containing moieties onto the surface of Ru particle supported on titania has been investigated, together with the effects of sodium on the activity and selectivity of titaniasupported $\mathrm{Ru}$ for Fischer-Tropsch synthesis. It is demonstrated that sodium facilitates the decoration and partial encapsulation of $\mathrm{Ru}$ by titanium-containing moieties as a consequence of the formation of sodium titanates. Since the Tammann temperature of the titanates is lower than that of the anatase or rutile phases of titania, the temperature at which titanium-containing moieties begin to migrate is reduced in the presence of sodium. The turnover frequency for $\mathrm{CO}$ consumption based on exposed Ru sites is observed to be independent of the sodium content of the catalyst, but the turnover frequency for methane formation decreases monotonically with increasing sodium content. The probability of chain growth and the olefin-to-paraffin ratio increase and the extent of ethylene reincorporation into the reaction products decreases with increasing sodium content.
These effects are attributed to a reduction in the surface concentration and mobility of $\mathrm{H}$ atoms adsorbed on $\mathrm{Ru}$.

4. Promotion of $\mathrm{CO}$ and $\mathrm{CO}_{2}$ Hydrogenation over $\mathrm{Rh}$ by Metal Oxides: The Influence of Oxide Lewis Acidity and Reducibility (Publication 7)

\section{A. Boffa, C. Lin, A.T. Bell, and G.A. Somorjai}

An investigation has been carried out of $\mathrm{CO}$ and $\mathrm{CO}_{2}$ hydrogenation over a $\mathrm{Rh}$ foil decorated with submonolayer quantities of $\mathrm{AlO}_{\mathrm{x}}, \mathrm{TiO}_{\mathrm{x}}, \mathrm{FeO}_{\mathrm{x}}, \mathrm{ZrO}_{\mathrm{x}}, \mathrm{NbO}_{\mathrm{x}}, \mathrm{TaO}_{\mathrm{x}}$, and $W_{x}$. The rate of methane formation was measured at $1 \mathrm{~atm}$, and the state of the working catalyst was characterized by XPS immediately after reaction. With the exception of $\mathrm{AlO}_{\mathrm{x}}$, each of the oxides was found to enhance the rate of $\mathrm{CO}$ methanation relative to that observed over unpromoted $\mathrm{Rh}$. The maximum degree of rate enhancement occurs at an oxide coverage of approximately half a monolayer. $\mathrm{AlO}_{\mathrm{x}}$ retards the formation of methane in direct proportion to the oxide coverage. In the case of $\mathrm{CO}_{2}$ hydrogenation, $\mathrm{FeO}_{\mathrm{x}}$ behaves in a manner identical to that of $\mathrm{AlO}_{\mathrm{x}}$, whereas all of the other oxides studied produce a maximum degree of methanation rate enhancement at an oxide coverage of half a monolayer. The enhancement of $\mathrm{CO}$ and $\mathrm{CO}_{2}$ hydrogenation is attributed to the formation of Lewis acid-base complexes between the oxygen end of adsorbed $\mathrm{CO}$ (or $\mathrm{H}_{2} \mathrm{CO}$ formed during the reaction) and anionic vacancies present at the edge of the oxide-metal boundary. The degree of enhancement of $\mathrm{CO}$ and $\mathrm{CO}_{2}$ hydroge nation is found to increase with the average oxidation state of the metal in the oxide overlayer. This trend is attributed to the direct relationship between Lewis acidity of an oxide and the oxidation state of the metal in the oxide. The degree of reduction of a given oxide is found to be higher during $\mathrm{CO}_{2}$ hydrogenation than $\mathrm{CO}$ hydrogenation.

\section{Work in Progress: Thermal Activation of $\mathrm{CH}_{4}$ to Produce $\mathrm{CH}_{\mathrm{x}}$ Species}

\section{J. Carstens and A.T. Bell}

The reaction of methane with $\mathrm{Ru} / \mathrm{SiO}_{2}$ is being investigated. Above $473 \mathrm{~K}$ methane adsorbs dissociatively on $\mathrm{Ru}$ to produce a spectrum of carbonaceous species. These species react at different rates with hydrogen during a temperature-programmed reduction experiment, to produce methane and a homologous series of higher molecular weight hydrocarbons. Four types of carbon have been identified thus far: atomic carbon, $\mathrm{CH}, \mathrm{C}_{2} \mathrm{H}_{\mathrm{x}}(\mathrm{x}=2$, 3 ), and graphitic carbon. Further structural characterization of these forms of carbon by means of ${ }^{13} \mathrm{C}$ NMR is in 
progress. The dynamics of $\mathrm{C}-\mathrm{C}$ bond formation by reaction of $\mathrm{CH}_{\mathrm{x}}$ species and the dynamics of $\mathrm{C}-\mathrm{C}$ bond rupture are being investigated as well, to determine the effects of $\mathrm{Ru}$ particle size and morphology, and the addition of elements such as $\mathrm{Cu}$ and $\mathrm{Re}$ onto the surface of the $\mathrm{Ru}$ particles. The objective of this work is to determine catalyst compositions and structures that will maximize the formation of reactive species from methane and their conversion to hydrocarbons and other organic compounds.

\section{Work in Progress: Solid-State Chemistry of Rh Supported on Alumina}

\section{Z. Weng-Sieh, R. Gronsky, and A.T. Bell}

The interactions of small $\mathrm{Rh}$ particles $(10-30 \AA)$ with $\gamma-\mathrm{Al}_{2} \mathrm{O}_{3}$ under reducing and oxidizing conditions are being investigated by means of atomic resolution transmission microscopy. Under reducing conditions the particles undergo progressive sintering, but there is no loss of $\mathrm{Rh}$ into the support. When the atmosphere in which the catalyst is heated is net oxidizing, the Rh particles are rapidly converted to $\mathrm{Rh}_{2} \mathrm{O}_{3}$ and then more slowly into $\mathrm{RhO}_{2}$ and $\mathrm{RhO}$. The latter two phase wet the surface of the support forming a very thin layer. With time the twodimensional rhodium oxide phases appear to react with the alumina, forming a mixed oxide phase that is very stable to reduction. The structure of this new phase is currently undefined. Under both reducing and oxidizing conditions, $\mathrm{Rh}$, catalyzes the conversion of $\gamma-\mathrm{Al}_{2} \mathrm{O}_{3}$ to $\delta$ - and $\theta$-, and finally to $\alpha-\mathrm{Al}_{2} \mathrm{O}_{3}$. These transformations are accompanied by a progressive loss in the support surface area, a process that facilitates the sintering of Rh particles.

\section{FY 1994 PUBLICATIONS AND REPORTS}

\section{Refereed Journals}

1. T. Komaya and A. T. Bell, "Estimates of Rate Coefficients for Elementary Processes Occurring during Fischer-Tropsch Synthesis over Ru/TiO 2 ," J. Catal. 146, 237 (1994); LBL34334.

2 R. M. Pittman and A. T. Bell, "Raman Investigation of $\mathrm{NH}_{3}$ Adsorption on $\mathrm{TiO}_{2}, \mathrm{Nb}_{2} \mathrm{O}_{5}$, and $\mathrm{Nb}_{2} \mathrm{O}_{5} / \mathrm{TiO}_{2}$," Catal. Lett 24, 1 (1994); LBL-34518.

3. J. Kritzenberger, H.C. Gaede, J.S. Shore, A. Pines, and A.T. Bell, " ${ }^{129} \mathrm{Xe}$ NMR Study of $\mathrm{TiO}_{2}$ (Anatase)-Supported $\mathrm{V}_{2} \mathrm{O}_{5}$ Catalysts," J. Phys. Chem. 98, 10173 (1994); LBL-35048.

4. D.B. Clarke, D.-K. Lee, M.J. Sandoval, and A.T. Bell, "Infrared Studies of the Mechanism of Methanol Decomposition on $\mathrm{Cu} / \mathrm{SiO}_{2}$," J. Catal. 150, 81 (1994); LBL35149.
5. T. Komaya, A.T. Bell, Z. Weng-Sieh, R. Gronsky, F. Engelke, T.S. King, and M. Pruski, "The Influence of Metal-Support Interactions on the Accurate Determination of $\mathrm{Ru}$ Dispersion for $\mathrm{Ru} / \mathrm{TiO}_{2}$ Catalysts," J. Catal. 149, 142 (1994); LBL-35150.

6. T. Komaya, A.T. Bell, Z. Weng-Sieh, R. Gronsky, F. Engleke, T.S. King, and M. Pruski, "Effects of Dispersion and Metal-Metal Oxide Interactions on Fischer-Tropsch Synthesis over $\mathrm{Ru} / \mathrm{TiO}_{2}$ and $\mathrm{TiO}_{2}$-Promoted $\mathrm{Ru} / \mathrm{TiO}_{2}$," J. Catal. 150, 400 (1994); LBL-35440.

7. A. B. Boffa, C. Lin, G. A. Somorjai, and A. T. Bell, "Lewis Acidity as an Explanation for Oxide Promotion of Metals: Implications of Its Importance for Catalytic Reactions," Catal. Lett. 27, 243 (1994); LBL-35762.

8. A.B. Boffa, C. Lin, G.A. Somorjai, and A.T. Bell, "Promotion of $\mathrm{CO}$ and $\mathrm{CO}_{2}$ Hydrogenation over $\mathrm{Rh}$ by Metal Oxides: The Influence of Oxide Lewis Acidity and Reducibility," J. Catal. 149, 149 (1994); LBL-34740.

\section{LBL Reports}

9. T. Komaya, A.T. Bell, Z. Weng-Sieh, R. Gronsky, F. Engleke, T.S. King, and M. Pruski, "Effects of Sodium on the Structure and Fischer-Tropsch Synthesis Activity of $\mathrm{Ru} / \mathrm{TiO}_{2}$," J. Catal.. (in press); LBL-35968.

10. D.B. Clarke and A.T. Bell, "An Infrared Study of Methanol Synthesis from $\mathrm{CO}_{2}$ on Clean and Potassium-Promoted $\mathrm{Cu} / \mathrm{SiO} 2$," J. Catal. (in press); LBL-36275.

11. A.B. Boffa, H.C. Galloway, P.W. Jacobs, J.J. Benitez, J.D. Batteas, M. Salmeron, A.T. Bell, and G.A. Somorjai, "The Growth and Structure of Titanium Oxide Films on Pt(111) Investigated by LEED, XPS, ISS, and STM," Surf. Sci. (in press); LBL-35763.

12. A.T. Bell, L.E. Manzer, N.Y. Chen, V.W. Weekman, L.L. Hegedus, and C.J. Pereira, "The Role of Catalysis in Environmental Protection," CEP (in press); LBL-33460.

13. A.T. Bell, "Understanding Catalysis at the Molecular Level," in Proceedings of the Second Tokyo Conference on Advanced Catalytic Science and Technology, Tokyo, Japan, August 21-26, 1994 (in press); LBL-36760.

\section{Other Publication}

14. A.T. Bell and A. Pines, Eds., "NMR Techniques in Catalysis," Marcel Dekker, Inc., New York, 1994.

Invited Talks

15. A.T. Bell, "Structure-Performance Relationships for $\mathrm{V}_{2} \mathrm{O}_{5} / \mathrm{TiO}_{2}$ Catalysts Used for SCR of NO," Department of Chemical Engineering, Brigham Young University, Provo, UT, December 2, 1993.

16. A.T. Bell, "Defining the Relationships Between Catalyst Structure and Performance: Progress and Perspectives," Reilly Lecture, Department of Chemical Engineering, Notre Dame, IN, April 12, 1994. 
17. A.T. Bell, "Identifying the Relationships Between Catalyst Properties and Catalyst Performance," CCR NIChE Conference, Palm Springs, CA, May 22-25, 1994.

18. A.T. Bell, "Applications of Transient-Response Methods to the Study of the Mechanism and Dynamics of Catalyzed Reactions," ACS Colloid and Surface Chemistry Symposium, Stanford Univeristy, Stanford, CA, June 1922, 1994

19. A.T. Bell, "Studies of the Structure and Promoter Effects of Metal Oxides Deposited on Transition Metal Catalysts," Department of Chemistry, Sophia University, Tokyo, Japan, August 8, 1994.

20. A.T. Bell, "Studies of the Structure and Promoter Effects of Metal Oxides Deposited on Transition Metal Catalysts," Department of Chemistry, Nagoya University, Nagoya, Japan, August 9, 1994.

21. A.T. Bell, "Studies of the Structure and Promoter Effects of Metal Oxides Deposited on Transition Metal Catalysts," Department of Chemistry, Tokyo Institute of Technology, Tokyo, Japan, August 11, 1994.

22. A.T. Bell, "Mechanistic Studies of Methanol Synthesis and Decomposition over Cu," Symposium on Recent Progress in Catalytic Chemistry, Sapporo, Japan, August 17-20, 1994.

23. A.T. Bell, "Understanding Heterogeneous Catalysis at the Molecular Level: Challenges and Perspectives," Second
Tokyo Conference on Advanced Catalytic Science and Technology, Tokyo, Japan, August 21-26, 1994.

\section{Contributed Talks}

24. A.T. Bell, "A Scheme for Metal Oxide Promotion of $\mathrm{CO}$ and $\mathrm{CO}_{2}$ Hydrogenation over $\mathrm{Rh}$ Based on the Correlation of Rate Enhancement and Oxidation State," ACS Meeting, San Diego, CA, March 13-18, 1994.

25. A.T. Bell, "Mechanistic Studies of Methanol Synthesis over $\mathrm{Cu} / \mathrm{SiO}_{2}$," ACS Meeting, San Diego, CA, March 13-18, 1994.

26. A.T. Bell, "Mechanistic Studies of Methanol Synthesis over $\mathrm{Cu} / \mathrm{SiO}_{2}$," Meeting of the California Catalysis Society, Lawrence Berkeley Laboratory, Berkeley, CA, April 28-29, 1994.

27. A.T. Bell, "Mechanistic Studies of Methanol Synthesis over $\mathrm{Cu} / \mathrm{SiO}_{2}$," AIChE Meeting, San Francisco, CA, November 13-18, 1994.

28. A.T. Bell, "Effects of Dispersion and Metal-Metal Oxide Interactions on Fischer-Tropsch Synthesis over $\mathrm{Ru} / \mathrm{TiO}_{2}$ and $\mathrm{TiO}_{2}$-Promoted Ru/SiO 2 ," AIChE Meeting, San Francisco, CA, November 13-18, 1994. 


\section{Transition-Metal-Catalyzed Conversion of $\mathrm{CO}, \mathrm{NO}, \mathrm{H}_{2}$ and Organic Molecules to Fuels and Petrochemicals*}

\author{
Robert G. Bergman, Investigator
}

\section{INTRODUCTION}

The goal of this program is the development of new chemical reactions in which transition metals interact with organic materials and the understanding of how these reactions work. In the early 1980 s workers on this project found the first complexes that undergo oxidative addition into the carbon-hydrogen bonds of completely saturated hydrocarbons $(\mathrm{M}+\mathrm{R}-\mathrm{H} \rightarrow \mathrm{R}-\mathrm{M}-\mathrm{H}$; often referred to as a " $\mathrm{C}-\mathrm{H}$ bond activation" reaction). These reactions involved iridium and rhodium in the relatively low formal +1 oxidation state [i.e., $\operatorname{Ir}(\mathrm{I})$ and $\mathrm{Rh}(\mathrm{I})]$. More recently an iridium $\mathrm{C}-\mathrm{H}$ activation reaction involving the more highly oxidized Ir(III) center has been discovered. Research is now being directed at examining the scope, selectivity, and mechanism of these processes, extending them to other systems, and developing ways to convert activated metal complexes $\mathrm{X}$ $\mathrm{M}-\mathrm{H}$ into functionalized organic molecules. During the current year substantial progress was made in two areas. In the $\mathrm{Rh}(\mathrm{I})$ work an extensive flash kinetic study (carried out in collaboration with Dr. C.B. Moore and his coworkers) of the $\mathrm{Rh}(\mathrm{I}) \mathrm{C}-\mathrm{H}$ activation reaction was completed. These experiments utilized liquefied noble gas solvents at low temperatures, and provided information about the reaction mechanism at a level of detail that had not been previously attainable. In the $\operatorname{Ir}(\mathrm{III})$ chemistry, the first experiments designed to carry out $\mathrm{C}-\mathrm{H}$ activation with induction of molecular asymmetry were carried out.

\section{IR Flash Kinetic Spectroscopy of C-H Bond} Activation of Cyclohexane- $d_{0}$ and $-d_{12}$ by $\mathrm{Cp} * \mathrm{Rh}(\mathrm{CO})_{2}$ in Liquid Rare Gases: Kinetics, Thermodynamics, and an Unusual Isotope Effect

\section{R.H. Schultz, A.A. Bengali, M.J. Tauber, B.H. Weiller, E.P. Wasserman, K.R. Kyle, C.B. Moore, and R.G. Bergman}

Flash kinetic spectroscopy with infrared detection is used to probe $\mathrm{C}-\mathrm{H}$ activation of cyclohexane- $d_{0}$ and $-d_{12}$ by intermediates generated upon ultraviolet irradiation of

*This work was supported by the Director, Office of Energy Research, Office of Basic Energy Sciences, Chemical Sciences Division of the U.S. Department of Energy under Contract No. DE-AC03-76SF00098.
$\mathrm{Cp} * \mathrm{Rh}(\mathrm{CO})_{2}\left(\mathrm{Cp}^{*}=\mathrm{C}_{5}\left(\mathrm{CH}_{3}\right)_{5}\right)$ in liquid rare gas $(\mathrm{Rg}=\mathrm{Kr}$ or Xe) solution at low temperature (163-193 K). Upon UV photolysis, a new C-O stretching band (at $1946.5 \mathrm{~cm}^{-1}$ in $\mathrm{Kr}$ and at $1941.5 \mathrm{~cm}^{-1}$ in Xe) appears promptly, which we attribute to $\mathrm{Cp} * \mathrm{Rh}(\mathrm{CO})(\mathrm{Rg})$. In the presence of hydrocarbon a second $\mathrm{C}-\mathrm{O}$ stretching band $\left(2002.5 \mathrm{~cm}^{-1}\right.$ in $\mathrm{Kr}$ and $1998 \mathrm{~cm}^{-1}$ in Xe) grows in at the same observed rate as the disappearance of the $1946.5 \mathrm{~cm}^{-1}$ band (Figure 1-1). We attribute this second band to the alkyl hydride product, $\mathrm{Cp} * \mathrm{Rh}(\mathrm{CO})(\mathrm{H})\left(\mathrm{C}_{6} \mathrm{H}_{11}\right)$. The concentration dependence of the observed reaction rate in $\mathrm{Kr}$ solution shows behavior consistent with a pre-equilibrium mechanism in which the initial $\mathrm{Cp} * \mathrm{Rh}(\mathrm{CO})(\mathrm{Rg})$ complex equilibrates with a weakly bound $\mathrm{Cp} * \mathrm{Rh}(\mathrm{CO})$ (alkane) complex (which has an IR carbonyl frequency unresolvable from that of the rare gas complex) followed by $\mathrm{C}-\mathrm{H}$ activation of the latter (Figure 1-2). We observe a large normal kinetic isotope effect $\left(k_{2}{ }^{\mathrm{H}} / k_{2} \mathrm{D} \approx 10\right)$ on the $\mathrm{C}-\mathrm{H}$ activation step $k_{2}$, but most unusually, a large inverse isotope effect on the preequilibrium constant $\left(K_{\mathrm{eq}}{ }^{\mathrm{H}} / K_{\mathrm{eq}} \mathrm{D} \approx 0.1\right)$, implying that $\mathrm{C}_{6} \mathrm{D}_{12}$ binds more strongly to the rhodium center than does $\mathrm{C}_{6} \mathrm{H}_{12}$. From the temperature dependence of $k_{2}$, we derive Eyring activation energies $\Delta \mathrm{H}^{\ddagger}$ of $4.2 \pm 0.5 \mathrm{kcal} / \mathrm{mol}$ for $k_{2}{ }^{\mathrm{H}}$ and $5.3 \pm 0.5 \mathrm{kcal} / \mathrm{mol}$ for $k_{2}{ }^{\mathrm{D}}$. A reaction coordinate/energy diagram comparing the changes in energy that occur during the $\mathrm{C}-\mathrm{H}(\mathrm{C}-\mathrm{D})$ activation process for both cyclohexane and cyclohexane- $d_{12}$ is illustrated in Figure 1-3).

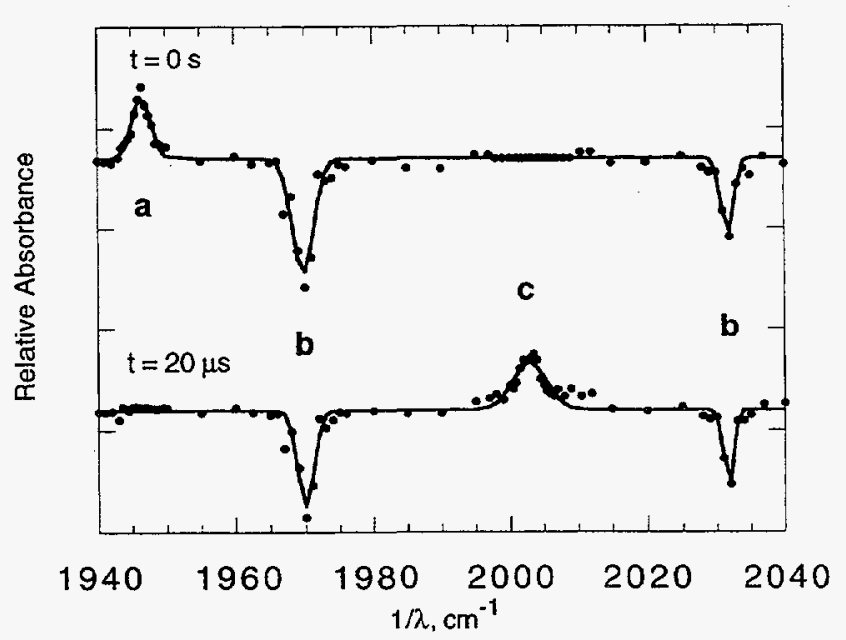

Figure 1-1. Time-dependent infrared spectrum of the photolysis of $\mathrm{CP}^{*} \mathrm{Rh}(\mathrm{CO})_{2}$ in liquid $\mathrm{Kr}$ in the presence of $0.01 \mathrm{M} \mathrm{C}_{6} \mathrm{H}_{12}$ at $183 \mathrm{~K}$. Shown are the relative absorbances at $\sim 0.05$ and $20 \mu \mathrm{s}$ after the photolysis pulse, normalized to the $\mathrm{Cp} * \mathrm{Rh}(\mathrm{CO})_{2}$ concentration at the time each wavelength data point was collected. The peak marked a corresponds to $\mathrm{Cp} * \mathrm{Rh}(\mathrm{CO}) \mathrm{Kr}$; the negative peaks marked $b$ correspond to the disappearance of parent $\mathrm{Cp}_{\mathrm{p}} \mathrm{Rh}(\mathrm{CO})_{2}$; the peak marked $\mathrm{c}$ corresponds to the product $\mathrm{CP}_{\mathrm{P}} * \mathrm{Rh}(\mathrm{CO})(\mathrm{H})\left(\mathrm{C}_{6} \mathrm{H}_{11}\right)$. 


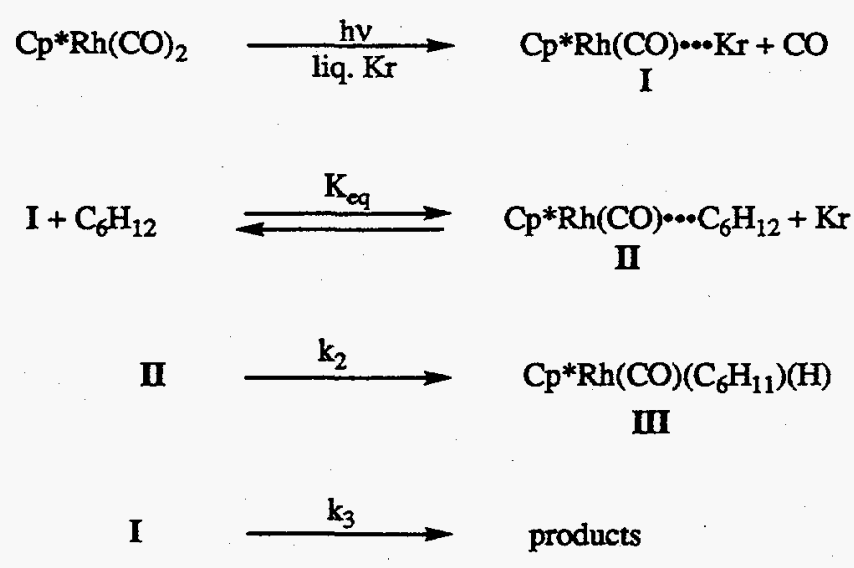

Figure 1-2. Proposed mechanism of cyclohexane $\mathrm{C}-\mathrm{H}$ bond activation by $\mathrm{Cp} * \mathrm{Rh}(\mathrm{CO})(\mathrm{Kr})$ in liquid krypton solution at low temperature.

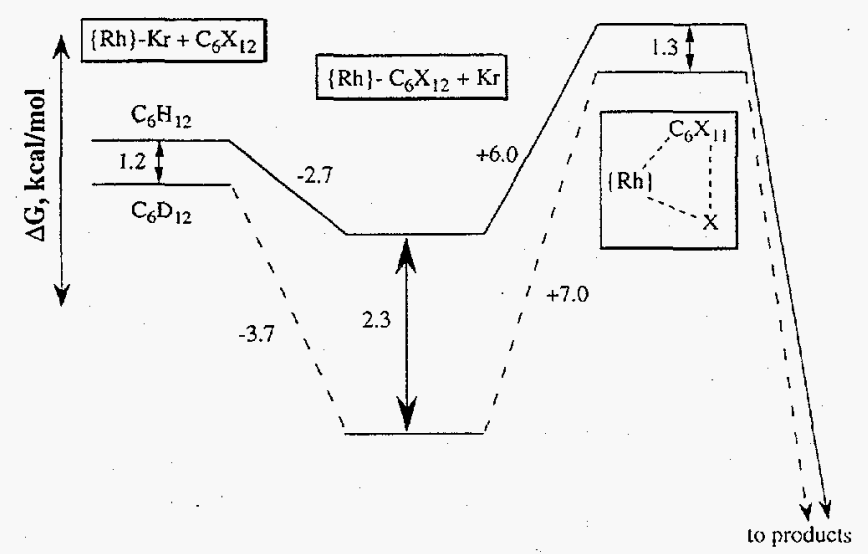

Figure 1-3. Reaction coordinate free energy diagram for activation of $\mathrm{C}_{6} \mathrm{H}_{12}$ and $\mathrm{C}_{6} \mathrm{D}_{12}$ by $\mathrm{Cp} * \mathrm{Rh}(\mathrm{CO})_{2}$ in liquid $\mathrm{Kr}$ at $183 \mathrm{~K}$. The reaction coordinate is assumed to be the $\mathrm{C}-\mathrm{H}$ or $\mathrm{C}-\mathrm{D}$ bond being broken.

2. Activation of the $\mathrm{C}-\mathrm{H}$ Bonds in Neopentane and Neopentane-d 12 by $\left(\eta^{5}-\mathrm{C}_{5}\left(\mathrm{CH}_{3}\right)_{5}\right) \mathrm{Rh}(\mathrm{CO})_{2}$ : Spectroscopic and Temporal Resolution of RhodiumKrypton and Rhodium-Alkane Complex Intermediates

\section{A.A. Bengali, R.H. Schultz, C.B. Moore, and R.G. Bergman}

The photoinitiated reaction of $\mathrm{Cp}^{*} \mathrm{Rh}(\mathrm{CO})_{2}\left(\mathrm{Cp}^{*}=\right.$ $\left(\eta_{5}-\mathrm{C}_{5} \mathrm{Me}_{5}\right)$ with neopentane $\left(\mathrm{C}\left(\mathrm{CH}_{3}\right)_{4}\right)$ and neopentane$d_{12}$ in liquid krypton has been studied using low temperature IR flash kinetic spectroscopy. Photolysis of $\mathrm{Cp}{ }^{*} \mathrm{Rh}(\mathrm{CO})_{2}$ generates a single transient absorption at $1946 \mathrm{~cm}^{-1}$ that is assigned to the $\mathrm{Cp} * \mathrm{Rh}(\mathrm{CO})(\mathrm{Kr})$ complex.
This complex reacts with neopentane to form the $\mathrm{C}-\mathrm{H}$ activated neopentyl hydride product $\mathrm{Cp} *(\mathrm{CO}) \mathrm{Rh}(\mathrm{H})$ $\left(\mathrm{CH}_{2} \mathrm{C}\left(\mathrm{CH}_{3}\right)_{3}\right)$ observed at $2008 \mathrm{~cm}^{-1}$. Confirming earlier flash kinetic results with cyclohexane, the results are consistent with a pre-equilibrium mechanism in which an initially formed transient krypton complex $\mathrm{Cp} * \mathrm{Rh}(\mathrm{CO})(\mathrm{Kr})$ is in rapid equilibrium with a transient (uninserted) alkane complex $\mathrm{Cp} * \mathrm{Rh}(\mathrm{CO})\left(\left(\mathrm{CH}_{3}\right)_{4} \mathrm{C}\right)$ which then proceeds to form the neopentyl hydride in a unimolecular step. Under most conditions our mechanism requires that both $\mathrm{Cp} * \mathrm{Rh}(\mathrm{CO})(\mathrm{Kr})$ and $\mathrm{Cp} * \mathrm{Rh}(\mathrm{CO})($ alkane) exhibit unresolved carbonyl stretching absorptions at $1946 \mathrm{~cm}^{-1}$. However, use of $\left(\mathrm{CD}_{3}\right)_{4} \mathrm{C}$ as the alkane substrate allows us, for the first time, to spectroscopically and temporally resolve both the rhodium-krypton and rhodium-alkane complex intermediates by $1 \mathrm{~cm}^{-1}$ (Figure 2-1), providing further support for the pre-equilibrium mechanism proposed to be operative in these systems. As with cyclohexane and cyclohexane- $d_{12}$, a normal isotope effect is observed for the unimolecular $\mathrm{C}-\mathrm{H}(\mathrm{D})$ insertion step but an unusual inverse equilibrium isotope effect (EIE) is measured for the pre-equilibrium step of the reaction: rhodium is bound an order of magnitude more strongly to $\left(\mathrm{CD}_{3}\right)_{4} \mathrm{C}$ than to $\left(\mathrm{CH}_{3}\right)_{4} \mathrm{C}$. A reaction coordinate/energy diagram comparing the changes in energy that occur during the $\mathrm{C}-\mathrm{H}$ activation process for both neopentane and neopentane-d 12 is illustrated in Figure 2-2.

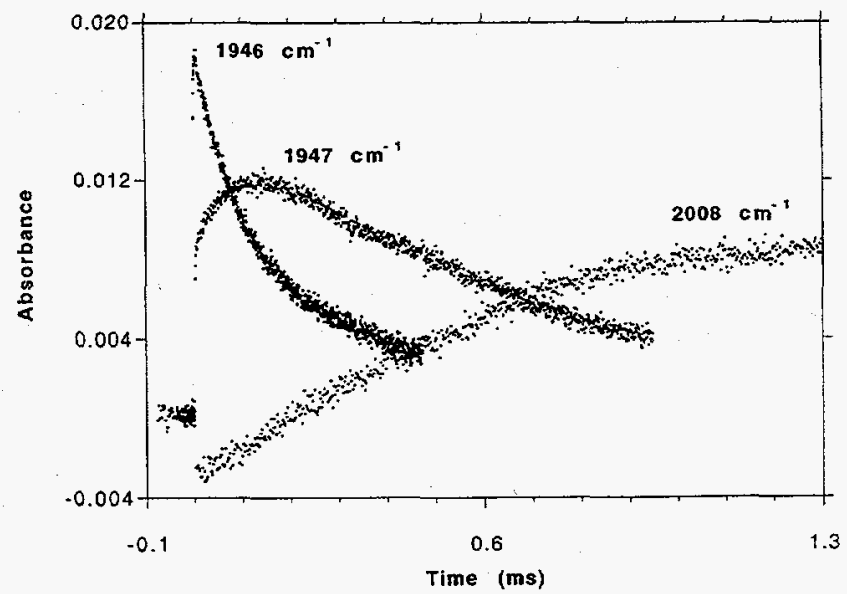

Figure 2-1. The time-dependent absorbances observed when $\mathrm{Cp}^{*} \mathrm{Rh}(\mathrm{CO})_{2}$ is photolyzed in liquid $\mathrm{Kr}$ at $165 \mathrm{~K}$ in the presence of $3.8 \mathrm{mM}$ neopentane- $d_{12}$. The transient at $1946 \mathrm{~cm}^{-1}$ shows biexponential behavior with a fast decay followed by a slower one. In marked contrast to the protiated system that shows only one transient absorption, a second transient absorption that grows in and then decays is observed at $1947 \mathrm{~cm}^{-1}$ in the deuterated system. The growth at $2008 \mathrm{~cm}^{-1}$ is assigned to the $\mathrm{Cp}^{*} \mathrm{Rh}(\mathrm{CO})(\mathrm{D})\left(\mathrm{C}_{5} \mathrm{D}_{11}\right)$ product. 


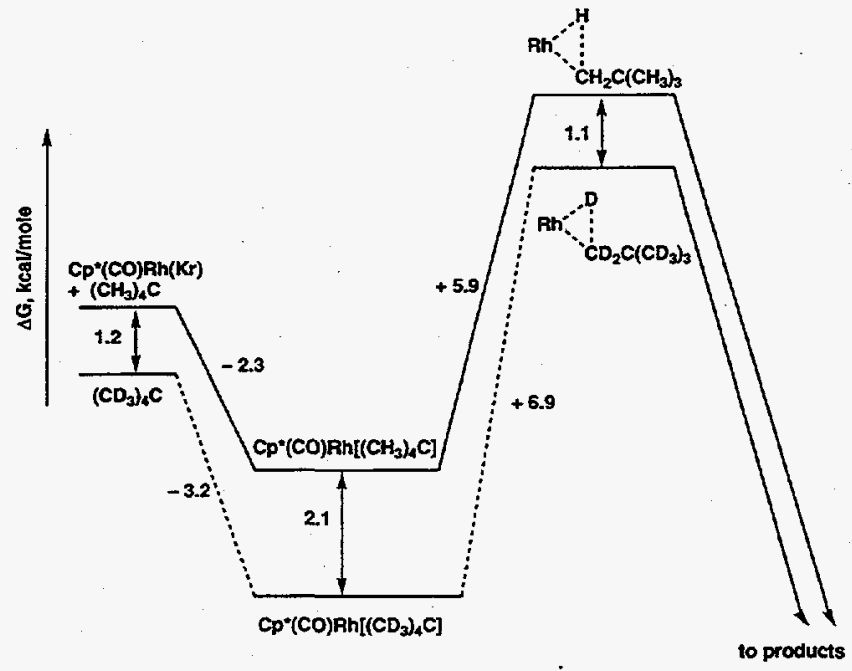

Figure 2-2. Reaction coordinate energy diagram for the activation of $\left(\mathrm{CH}_{3}\right)_{4} \mathrm{C}$ and $\left(\mathrm{CD}_{3}\right)_{4} \mathrm{C}$ by $\mathrm{Cp}{ }^{*} \mathrm{Rh}(\mathrm{CO})_{2}$ in liquid $\mathrm{Kr}$ at $165 \mathrm{~K}$ The reaction coordinate is assumed to be the $\mathrm{C}-\mathrm{H}(\mathrm{D})$ bond being broken.

3. Synthesis and Reactions of Enantiopure

Neomenthylcyclopentadienyliridium(III) Halides with Silver Salts. A Dramatic Steric Effect on the Competition Between Inter- and Intramolecular $\mathrm{C}-\mathrm{H}$ Activation

\section{Y. Ma and R.G. Bergman}

Enantiomerically resolved organometallic complexes have become increasingly useful in mechanistic studies and in applications to organic synthesis. To look for ways to extend chiral chemistry to $\mathrm{C}-\mathrm{H}$ activation, we have begun to explore the development of pseudotetrahedral iridiumand rhodium-based $\mathrm{C}-\mathrm{H}$ activating systems containing enantiomerically resolved ligands. Our initial goals are the development of useful methods for preparing such systems and determining the configurational stability of the chiral metal centers.

As the first of these results, we have found a surprising intramolecular $\mathrm{C}-\mathrm{H}$ activation/dehydrogenation reaction that leads to insignificant amplification of chirality at the Ir center in the product. The systems that we have prepared are complexes 1a, 4a and 10a, illustrated in Figure 3-1, in which an enantiomerically pure neomenthyl (NM) group is attached to the cyclopentadienyl $(\mathrm{Cp})$ ring. Reactions of enantiomerically pure $(\mathrm{NMCp}) \mathrm{M}\left(\mathrm{PMe}_{3}\right)(\mathrm{Me}) \mathrm{X}(\mathrm{M}=\mathrm{Rh}$, Ir) with silver saits produce complexes $\mathbf{2 a - 2 d}$, as shown in the figure. One of these cyclometallation products was

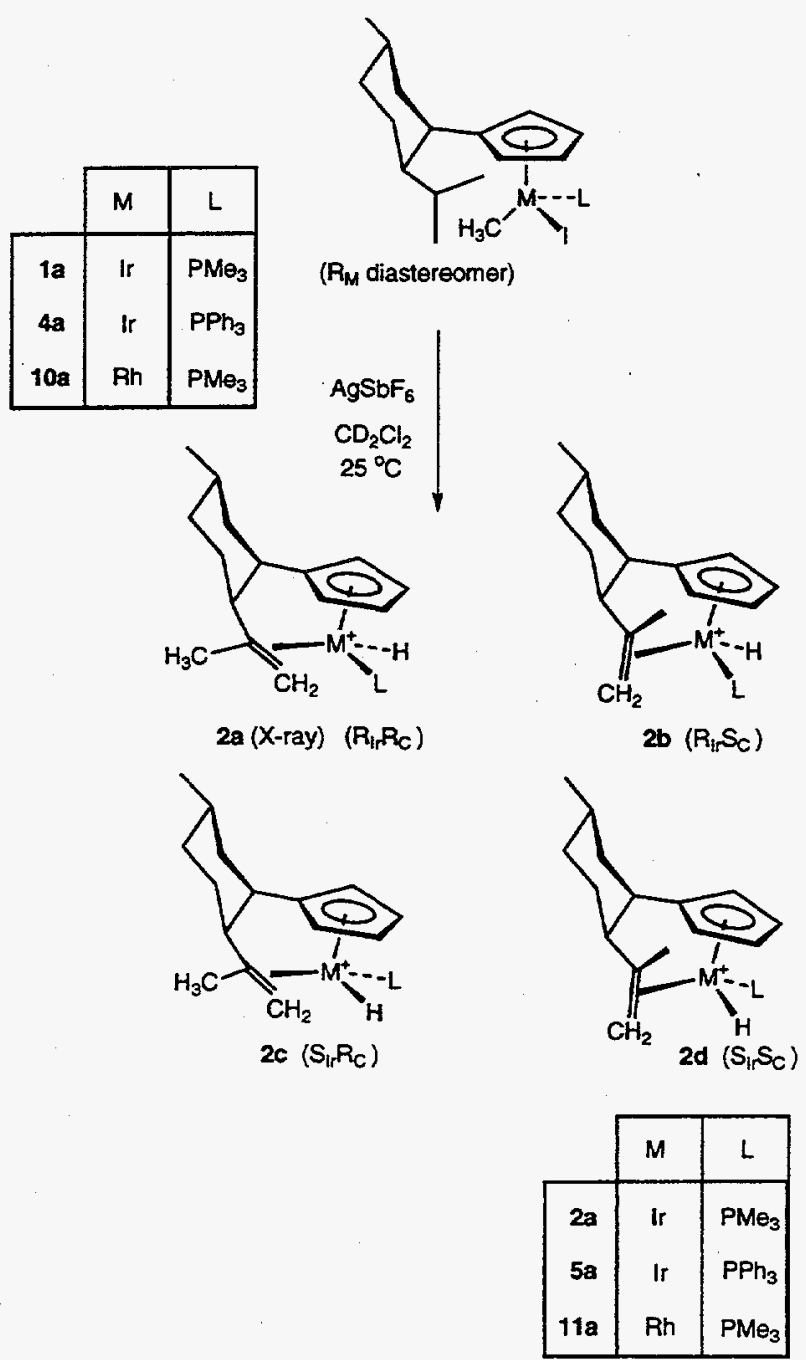

Figure 3-1. Summary of products formed by treatment of 1a with $\mathrm{AgSbF}_{6}$ in $\mathrm{CD}_{2} \mathrm{Cl}_{2}$ at $25^{\circ} \mathrm{C}$.

characterized by $x$-ray diffraction (Figure $3-2$ ). The fact that all four complexes are formed requires that the 16electron ( $\mathrm{NMCp}) \mathrm{M}\left(\mathrm{PMe}_{3}\right)(\mathrm{Me})^{+}$intermediates (e.g., 3, Figure 3-3) involved in these reactions are achiral (planar) at the metal centers on the time average. In contrast to the behavior of $(\mathrm{NMCp}) \mathrm{M}\left(\mathrm{PMe}_{3}\right)(\mathrm{Me})^{+}$, which exhibits only intramolecular $\mathrm{C}-\mathrm{H}$ activation involving the neomenthyl group, generation of the presumed intermediate (NMCp) $\operatorname{Ir}\left(\mathrm{PMe}_{3}\right)$ by photolysis of $(\mathrm{NMCp}) \operatorname{Ir}\left(\mathrm{PMe}_{3}\right)(\mathrm{H})_{2}$ in the presence of hydrocarbons results in intermolecular $\mathrm{C}-\mathrm{H}$ activation. The different outcomes of these two systems are attributed to steric energy differences in the transition states based on molecular mechanics calculations. 


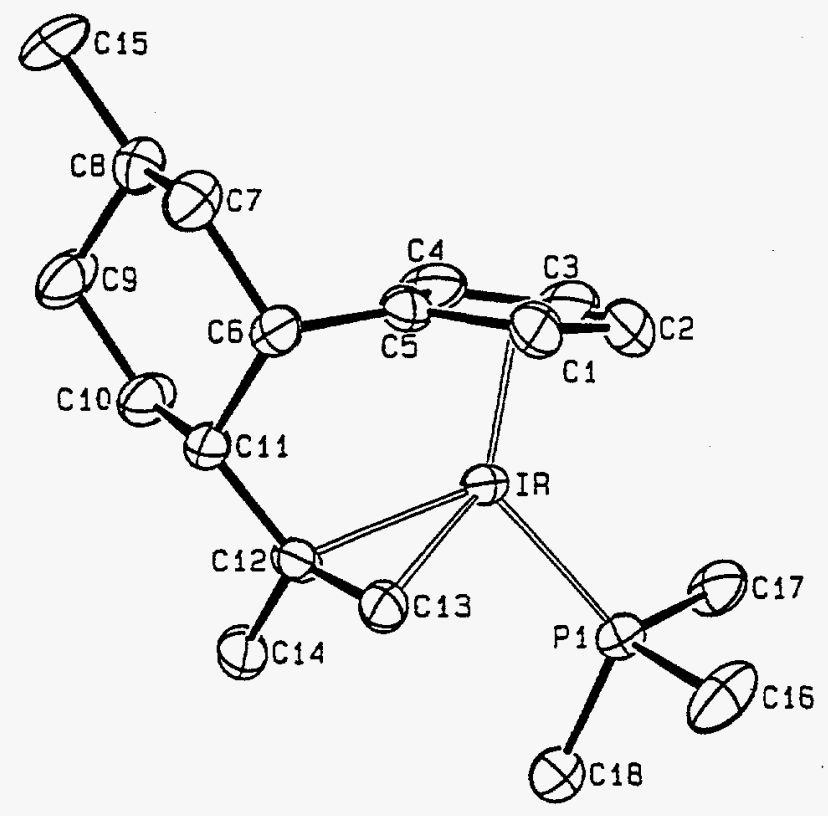

Figure 3-2. ORTEP drawing of 2a. Selected bond distances $(\AA)$ and angles $\left({ }^{\circ}\right)$ : Ir-Cp, 1.868; Ir-P, $2.277(2)$; Ir- $\mathrm{C}_{12}, 2.119(9)$; Ir- $\mathrm{C}_{13}$, 2.182(9); $\mathrm{C}_{12}-\mathrm{C}_{13}, 1.378(12) ; \mathrm{Cp}-\mathrm{Ir}-\mathrm{P}_{1}, 130.4 ; \mathrm{Cp}-\mathrm{Ir}-\mathrm{C}_{13}, 118.1 ; \mathrm{C}_{12}-\mathrm{Ir}-$ $P_{1}, 111.00(22) ; C_{13}$-Ir- $P_{1}, 90.11(25) ; C_{13}-$ Ir $-C_{12}, 36.7(3)$.
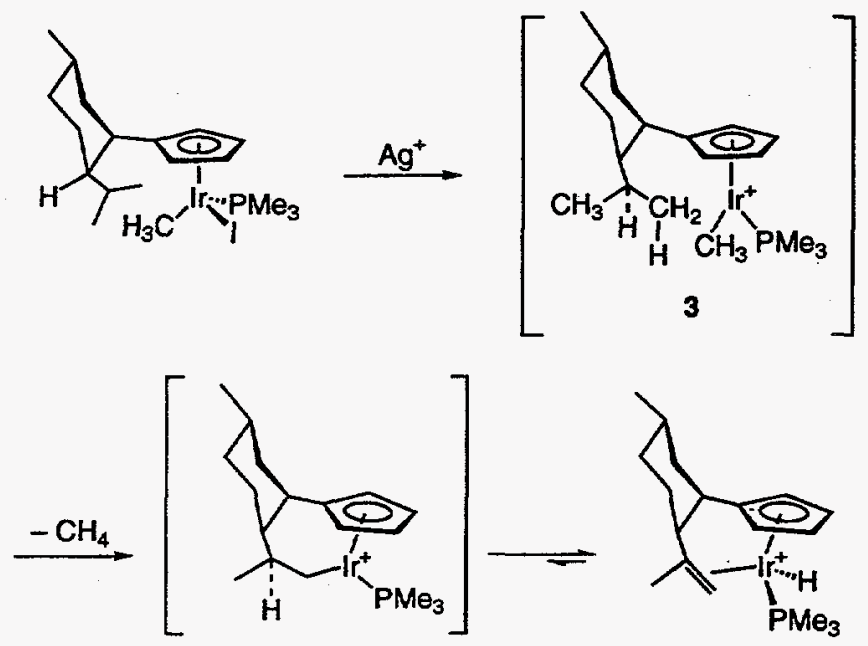

Figure 3-3. Proposed mechanism for the conversion of $1 \mathrm{a}$ to complexes 2.

\section{Work in Progress}

Research is now under way in the following areas: (1) Our flash kinetic investigations of $\mathrm{Rh}(\mathrm{I}) \mathrm{C}-\mathrm{H}$ activating intermediates in noble gas solutions are being extended to systems containing other metals and other organic substrates. Currently under investigation are the reactions of $\mathrm{Cp}{ }^{*} \mathrm{Rh}(\mathrm{CO})(\mathrm{Kr})$ with silanes and the reactions of the analogous cobalt complexes $\mathrm{CpCo}(\mathrm{CO})(\mathrm{Kr})$ with alkanes. (2) In the chiral $\mathrm{C}-\mathrm{H}$ activation area, new substituted cyclopentadienyl ligands are being designed to allow the synthesis of $\mathrm{Rh}$ and Ir complexes having planar chirality, with the goal of using these to obtain efficient asymmetric $\mathrm{C}-\mathrm{H}$ activating reactions.

\section{FY 1994 PUBLICATIONS AND REPORTS}

\section{Refereed Journals}

1. R.H. Schultz, A.A. Bengali, M.J. Tauber, B.H. Weiller, E.P. Wasserman, K.R. Kyle, C.B. Moore, and R.G.Bergman, "IR Flash Kinetic Spectroscopy of C-H Bond Activation of $d_{0-}$ and $d_{12}$-Cyclohexane by $\mathrm{Cp} * \mathrm{Rh}(\mathrm{CO})_{2}$ in Liquid Rare Gases: Kinetics, Thermodynamics, and an Unusual Isotope Effect," J. Am. Chem. Soc. 116, 7369 (1994); LBL-35543.

2. A.A. Bengali, R.H. Schultz, C.B. Moore, and R.G. Bergman, "Activation of the $\mathrm{C}-\mathrm{H}$ Bonds in Neopentane and Neopentane- $d_{12}$ by $\left(\eta^{5}-\mathrm{C}_{5}\left(\mathrm{CH}_{3}\right)_{5}\right) \mathrm{Rh}(\mathrm{CO})_{2}$ : Spectroscopic and Temporal Resolution of Rhodium-Krypton and Rhodium-Alkane Complex Intermediates," J. Am. Chem. Soc. 116, 9585 (1994); LBL-35544.

3. Y. Ma and R.G. Bergman, "Synthesis and Reactions of Enantiopure(Neomenthylcyclopentadienyl)iridium(III) $\mathrm{Hal}$ ides with Silver Salts. A Dramatic Steric Effect on the Competition Between Inter- and Intramolecular C-H Activation," Organometallics 13, 2548 (1994); LBL-35545.

\section{Invited Talks}

4. R.G. Bergman, "The Use of Organotransition Metal Complexes in the Formation and Cleavage of CarbonHydrogen and Carbon-Heteroatom Bonds in Organic Molecules," University of New Orleans, LA, October 22, 1993.

5. R.G. Bergman, "A New Intermolecular C-H Activation Reaction of Methane and Other Hydrocarbons with Ir(III) Complexes," Shell Development Company, Houston, TX, October 27, 1993.

6. R.G. Bergman, "Stoichiometric and Catalytic Reactions of Organic Compounds Mediated by Complexes with MetalNitrogen Multiple Bonds," University of Canterbury, Christchurch, New Zealand, December 1, 1993.

7. R.G. Bergman, "A New Intermolecular C-H Activation Reaction of Methane and Other Hydrocarbons with Ir(III) Complexes," New Zealand Institute of Chemistry Conference, Auckland, New Zealand, December 7, 1993.

8. R.G. Bergman, "Facile Intermolecular C-H Activation of Methane and Other Organic Compounds with Ir(III) Complexes," American Chemical Society Meeting, San Diego, CA, March 16, 1994.

9. R.G. Bergman, "The Use of Organotransition Metal Complexes to Activate Carbon-Hydrogen Bonds in Alkanes and Other Organic Molecules," Washington University, St. Louis, MO, March 31, 1994. 
10. R.G. Bergman, "The Use of Organotransition Metal Complexes to Activate Carbon-Hydrogen Bonds in Alkanes and Other Organic Molecules," University of MissouriColumbia, Columbia, MO, April 1, 1994.

11. R.G. Bergman, "Synthesis and Chemistry of Complexes with Transition-Metal Heteroatom Bonds," National Tsing Hua University, Taipei, Taiwan, April 18, 1994.

12. R.G. Bergman, "Activation of Carbon-hydrogen Bonds in Alkanes and Other Organic Molecules Using Organotransition Metal Complexes" National Chung Cheng University, Chia-Yi, Taiwan, April 19, 1994.

13. R.G. Bergman, "Activation of Carbon-hydrogen Bonds in Alkanes and Other Organic Molecules Using Organotransition Metal Complexes," Academica Sinica, Taipei, Taiwan, April 21, 1994.

14. R.G. Bergman, "Stoichiometric and Catalytic Reactions of Early-late Heterobinuclear Transition Metal Complexes," National Taiwan University, Taipei, Taiwan, April 22, 1994.
15. R.G. Bergman, "Activation of Carbon-Hydrogen Bonds in Alkanes and Other Organic Molecules with Organotransition Metal Complexes," Statoil Symposium, Oslo, Norway, May 30, 1994.

16. R.G. Bergman, "Reactions of Transition Metal Imido Complexes with Organic Compounds," Union Carbide, Bound Brook, NJ, June 20, 1994.

17. R.G. Bergman, "Reactions of Transition Metal Imido Complexes with Organic Compounds," DuPont, Wilmington, DE, June 21, 1994.

18. R.G. Bergman, "Activation of $\mathrm{C}-\mathrm{H}$ bonds in Alkanes and Other Organic Molecules Using $\operatorname{Ir}(\mathrm{I})$ and $\operatorname{Ir}(\mathrm{III})$ Complexes," International Conference on Coordination Chemistry, Kyoto, Japan, July 26, 1994.

19. R.G. Bergman, "Activation of $\mathrm{C}-\mathrm{H}$ bonds in Alkanes and Other Organic Molecules Using $\operatorname{Ir}(\mathrm{I})$ and $\operatorname{Ir}(\mathrm{III})$ Complexes," Tokyo Institute of Technology, Tokyo, Japan, July $27,1994$. 


\section{New Molecular Precursors for Low- Temperature Routes to Oxide Materials*}

\author{
T. Don Tilley, Investigator
}

\section{INTRODUCTION}

Advanced solid-state materials with useful properties increasingly involve intricate 3-dimensional networks, characterized by complex stoichiometries (e.g., in ceramic superconductors such as $\mathrm{HgBa}_{2} \mathrm{Ca}_{2} \mathrm{Cu}_{3} \mathrm{O}_{8+\delta}$ ) and/or metastable architectures (e.g., in zeolites). New generations of materials will undoubtedly result from chemically directed, low-temperature synthetic routes. Our approach involves use of synthesis, coordination chemistry, and condensation reactions for construction of novel 3-dimensional networks. Primary targets have been oxide-based materials, which are derived from tailored, oxygen-rich precursor molecules. Initial directions are based on metal complexes of the siloxide ligand $\mathrm{OSi}\left(\mathrm{O}^{t} \mathrm{Bu}\right)_{3}$ and the phosphate ligand $\mathrm{O}_{2} \mathrm{P}\left(\mathrm{O}^{\mathrm{t}} \mathrm{Bu}\right)_{2}$, which eliminate isobutylene and water cleanly at remarkably low temperatures $\left(100-200^{\circ} \mathrm{C}\right)$ to form $\mathrm{M}_{\mathrm{x}} \mathrm{Si}_{\mathrm{y}} \mathrm{O}_{z}$ or $\mathrm{M}_{\mathrm{x}} \mathrm{P}_{\mathrm{y}} \mathrm{O}_{\mathrm{z}}$ materials. The chemistry of this network formation and the homogeneity of the systems allow some control over phase transformations at higher temperatures. These "pyrolytic polymerization" reactions are being carried out in organized media to generate ordered microstructures for the resulting oxide materials. Further attempts to add directionality to the network formation, via added templates or "ancillary" ligands in the precursor, are expected to orient the condensation reactions. The ultimate goal of this research is to provide tailored materials with novel or specific structural, electronic, optical, and/or catalytic properties.

\section{Trialkoxysiloxy Complexes as Precursors to $\mathrm{MO}_{2} \cdot 4 \mathrm{SiO}_{2}(\mathrm{M}=\mathrm{Ti}, \mathrm{Zr}, \mathrm{Hf})$ Materials}

\section{K.W. Terry and T.D. Tilley}

We have found that tri(tert-butoxy)siloxy complexes $\mathrm{M}\left[\mathrm{OSi}\left(\mathrm{O}^{\mathrm{t} B u}\right)_{3}\right]_{4}(\mathbf{1}, \mathrm{M}=\mathrm{Ti} ; \mathbf{2}, \mathrm{M}=\mathrm{Zr} ; \mathbf{3}, \mathrm{M}=\mathrm{Hf})$ are lowtemperature precursors to $\mathrm{MO}_{2} \cdot 4 \mathrm{SiO}_{2}$ materials. ${ }^{1}$ These compounds decompose sharply, at ca. $250^{\circ} \mathrm{C}$ for 1 and at ca. $140^{\circ} \mathrm{C}$ for both 2 and 3 , with elimination of stoichiometric quantities of water and isobutylene (Figure 1-1). The $\mathrm{TiO}_{2} \cdot 4 \mathrm{SiO}_{2}$ material is amorphous to $1000^{\circ} \mathrm{C}$, and

*This work was supported by the Director, Office of Energy Research, Office of Basic Energy Sciences, Chemical Sciences Division, of the U.S. Department of Energy under Contract No. DE-AC03-76SF00098. samples taken to $1400^{\circ} \mathrm{C}$ contain anatase, rutile, and cristobalite. The $\mathrm{ZrO}_{2} \cdot 4 \mathrm{SiO}_{2}$ material is amorphous to ca. $1100^{\circ} \mathrm{C}$, where crystallization of tetragonal zirconia (t$\left.\mathrm{ZrO}_{2}\right)$ is apparent. Samples taken to $1200^{\circ} \mathrm{C}$ contain small $(6-21 \mathrm{~nm})$ crystallites of $\mathrm{t}-\mathrm{ZrO}_{2}$. Further thermolysis to $1500^{\circ} \mathrm{C}$ for $6 \mathrm{~h}$ results in a mixture of monoclinic zirconia $\left(\mathrm{m}-\mathrm{ZrO}_{2}\right.$ ), $\mathrm{t}-\mathrm{ZrO}_{2}$, and cristobalite (no $\mathrm{ZrSiO}_{4}$ was observed). Transmission electron microscopic analysis of $\mathrm{ZrO}_{2} \cdot 4 \mathrm{SiO}_{2}$ that had been annealed at $800^{\circ} \mathrm{C}$ revealed a relatively ordered, interpenetrating network of thin fibers. The $\mathrm{HfO}_{2} \cdot 4 \mathrm{SiO}_{2}$ material obtained by thermolysis of solid 3 possesses a more particulate (rather than fibrous) microstructure, and is amorphous until ca. $1000^{\circ} \mathrm{C}$, where t$\mathrm{HfO}_{2}$ (or c- $-\mathrm{HfO}_{2}$ ) starts to crystallize. Samples of $\mathrm{HfO}_{2} \cdot 4 \mathrm{SiO}_{2}$ taken to $1460^{\circ} \mathrm{C}$ contain $\mathrm{t}-\mathrm{HfO}_{2}$ (or cubic hafnia, c- $\mathrm{HfO}_{2}$ ), $\mathrm{m}-\mathrm{HfO}_{2}$ (in roughly equal amounts), and cristobalite. Decompositions of $\mathbf{2}$ and $\mathbf{3}$ in refluxing hydrocarbon solvents produce viscous, gel-like solutions composed of $\left[\mathrm{MSi}_{4} \mathrm{O}_{\mathrm{x}}(\mathrm{OH})_{\mathrm{y}}\right]_{\mathrm{z}}$ networks. Removal of solvent yields $\mathrm{MSi}_{4} \mathrm{O}_{\mathrm{x}}(\mathrm{OH})_{\mathrm{y}}$ powders (for $\mathrm{M}=\mathrm{Zr}$, the $\mathrm{BET}$ surface area is $520 \mathrm{~m}^{2} \mathrm{~g}^{-1}$ ) that display crystallization behaviors analogous to materials obtained by decomposing the solid compounds. Finally, relatively smooth, crack-free thin films may be spun onto quartz from $1 \%$ hydrocarbon solutions of 2 or 3 .

Recent studies on the solution-phase network formation have revealed a somewhat different stoichiometry. Thermolysis in toluene- $d_{8}$ shows that tert-butanol is also formed, and that a final product ratio of ca. 3:1 isobutylene to butanol is obtained. Furthermore, monitoring this reaction by ${ }^{1} \mathrm{H}$ NMR spectroscopy has revealed mechanistic information. Early in the transformation, an equivalent of $\mathrm{HOSi}\left(\mathrm{O}^{\prime} \mathrm{Bu}\right)_{3}$ is released per mol of 2 , and this is accompanied by formation of a new zirconium siloxide cluster complex that probably contains $\mu$-oxo or $\mu$ $\mathrm{O}_{2} \mathrm{Si}\left(\mathrm{O}^{t} \mathrm{Bu}\right)_{2}$ bridges. Kinetic and mechanistic studies on

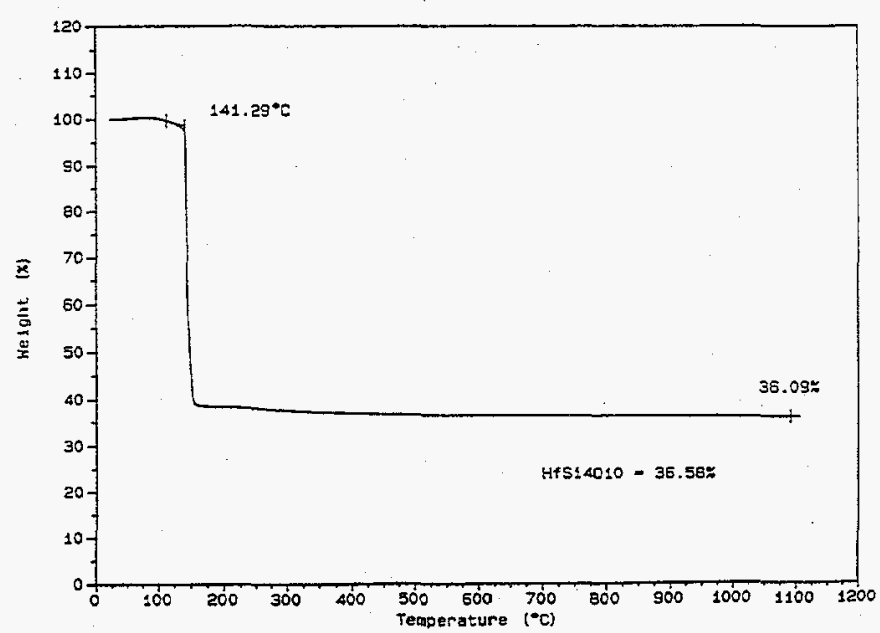

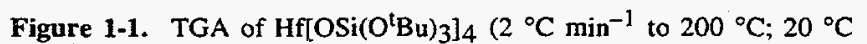
$\min ^{-1}$ to $1100^{\circ} \mathrm{C}$ ). 
elimination reactions of 2 indicate that the decomposition is an autocatalytic process, catalyzed by Brønsted acids. In benzene- $d_{6}$, these reactions proceed completely to a freelyflowing solution containing isobutylene, water, $t$-butanol, and a soluble, zirconia-silica $\mathrm{Zr}_{\mathrm{w}} \mathrm{Si}_{\mathrm{x}} \mathrm{O}_{\mathrm{y}}(\mathrm{OH})_{\mathrm{z}}$ sol, which upon further heating gives a transparent gel.

The high-temperature transformations of $\mathrm{MO}_{2} \cdot 4 \mathrm{SiO}_{2}$ $(\mathrm{M}=\mathrm{Zr}, \mathrm{Hf}$ ) obtained from both solid state and solution thermolyses have been studied in detail. Eventually, at very high temperatures, segregation into nanosized zirconia particles in a silica matrix occurs. It is clear from these results that in this system, amorphous and tetragonal zirconia are matrix-stabilized to an inordinate degree. Thermolysis of $\mathrm{ZrO}_{2} \cdot 4 \mathrm{SiO}_{2}$ at $1500^{\circ} \mathrm{C}$ for $6 \mathrm{~h}$ under argon results in a material that consists of a 9:1 ratio of tetragonal- to monoclinic-zirconia, and cristobalite after cooling to room temperature. Pure tetragonal zirconia is normally stable only over the temperature range of $950-2370^{\circ} \mathrm{C}$. We believe that these solid-state effects result from the highly homogeneous network formation that is accomplished with use of the "single source" molecular precursors.

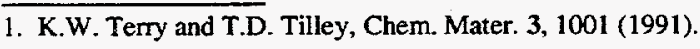

\section{Low-Temperature Pyrolytic Transformations of Tri(tert-butoxy)siloxy Derivatives of Aluminum to Aluminosilicate Materials}

\section{K.W. Terry, P.K. Gantzel, and T.D. Tilley}

Aluminosilicates are technologically important as catalysts, catalyst supports, and structural materials. Development of new chemical and physical properties for such materials will rely heavily on discovery of new routes to aluminosilicate networks. Low-temperature routes, such as those based on hydrolysis of alkoxides in solution (the sol-gel process), are particularly attractive since they offer the possibility for providing new (metastable) structures, and sol-gel routes to $\mathrm{Al}_{2} \mathrm{O}_{3}-\mathrm{SiO}_{2}$ systems based on cohydrolysis of aluminum and silicon compounds have been studied extensively. A major challenge in developing solgel type processes is to maintain homogeneity during the network-forming reactions. The "single source" precursor approach can lead to gels that are more homogeneous than those obtained by hydrolyzing $\mathrm{Al}(\mathrm{OR})_{3} / \mathrm{Si}\left(\mathrm{OR}^{\prime}\right)_{4}$ mixtures, but in aqueous media, Al-O-Si bridges are hydrolyzed rapidly in the initial phase of the condensation. To achieve a high degree of homogeneity at low temperatures, we have been examining aluminum-silicon precursor compounds that undergo thermolytic conversions to silicate materials. ${ }^{1}$

The tri(tert-butoxy)siloxy complex $\left\{\mathrm{Me}_{2} \mathrm{Al}[\mu\right.$ $\left.\left.\mathrm{OSi}\left(\mathrm{O}^{t} \mathrm{Bu}\right)_{3}\right]\right\}_{2}$ (4) was prepared by the reaction of $\left[\mathrm{AlMe}_{3}\right]_{2}$ with $\mathrm{HOSi}\left(\mathrm{O}^{t} \mathrm{Bu}\right)_{3}$. Molecules of 4 in the crystal have a folded $\mathrm{Al}_{2} \mathrm{O}_{2}$ core $\left(156.2^{\circ}\right.$ ), probably because of steric interactions involving the bulky siloxy groups (Figure 2-1). A trimeric derivative, $\left[\mathrm{Me}\left(\mathrm{HuO}^{\mathrm{Bu}} \mathrm{AlOSi}\left(\mathrm{O}^{\mathrm{T}} \mathrm{Bu}\right)_{3}\right]_{3}(5)\right.$, was obtained by addition of tert-butanol to 4 . Compounds 4 and 5 were investigated as precursors to aluminosilicate materials. In the solid state, 4 undergoes thermolysis at low temperatures $\left(150-200^{\circ} \mathrm{C}\right)$ by cleanly eliminating isobutylene (6 equiv), methane, and water. Clean conversion to a $\mathrm{Al}_{2} \mathrm{O}_{3} \cdot 2 \mathrm{SiO}_{2}$ material is observed by thermal gravimetric analysis (TGA) and elemental analysis. Differential thermal analysis (DTA) and x-ray powder diffraction (XRD) show that mullite crystallizes at about $1000^{\circ} \mathrm{C}$, and at $1200^{\circ} \mathrm{C}$, mullite particles of about $15 \mathrm{~nm}$ are produced (as calculated from the Scherrer equation). Compound 5 exhibits similar pyrolytic conversions, except that much higher surface areas are observed for the aluminosilicates produced. For example, thermolysis of 5 at $200^{\circ} \mathrm{C}$ gives a sample with a B.E.T. surface area of $270 \mathrm{~m}^{2} \mathrm{~g}^{-1}$, compared to a surface area of only $30 \mathrm{~m}^{2} \mathrm{~g}^{-1}$ for a sample obtained similarly from 4 . Compound 5 also cleanly decomposes by elimination of isobutylene (12 equiv). The network-forming thermolyses of $\mathbf{4}$ and $\mathbf{5}$ are facile enough to be conveniently carried out in solution. For example, 4 is pyrolyzed in refluxing toluene, resulting in a polymerization that resembles the sol-gel process. A gel powder obtained from this procedure had a surface area of $210 \mathrm{~m}^{2} \mathrm{~g}^{-1}$, and was composed of small particles (ca. 500 $\mathrm{nm}$ in diameter) consisting of much smaller grains (by transmission electron microscopy, TEM). Heating this sample to $800^{\circ} \mathrm{C}$ increased the B.E.T. surface area to $280 \mathrm{~m}^{2} \mathrm{~g}^{-1}$, and further heating to $1200^{\circ} \mathrm{C}$ produced mullite particles (average size $18 \mathrm{~nm}$, by XRD) that were densely packed into an amorphous silica matrix (by TEM).

1. K.W. Terry, P.K. Gantzel, and T.D. Tilley, Chem. Mater. 4, 1290 (1992).

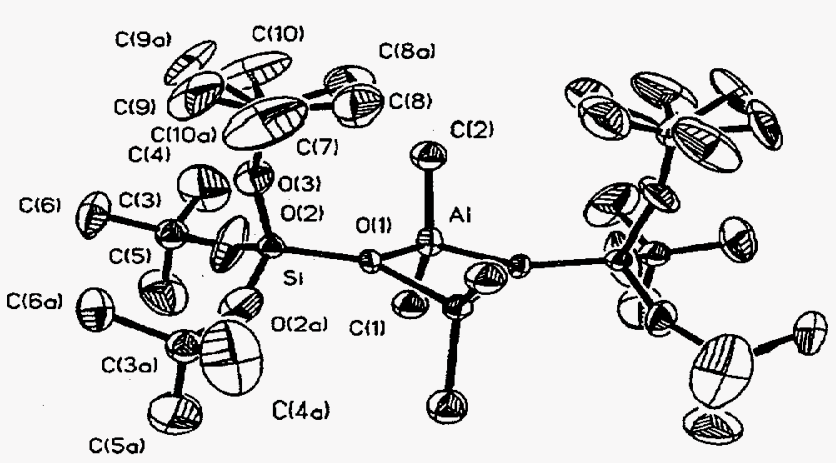

Figure 2-1. ORTEP view of $\left\{\mathrm{Me}_{2} \mathrm{Al}\left[\mu-\mathrm{OSi}\left(\mathrm{O}^{t} \mathrm{Bu}\right)_{3}\right]\right\}_{2}$ showing disordered, half-atom occupancies for methyl carbon atoms. 
3. Chromium(II) and Chromium(III) Tri-tertbutoxysiloxy Complexes

\section{K.W. Terry, P.K. Gantzel, and T.D. Tilley}

The pyrolytic polymerization of $\left\{\mathrm{Zr}\left[\mathrm{OSi}\left(\mathrm{O}^{t} \mathrm{Bu}\right)_{3}\right]_{4}\right\}_{2}$ produces high surface area, amorphous zirconium silicates, which segregate upon further heating to nanosize zirconia particles dispersed in a silica matrix. This observation suggested the possible use of this methodology in new syntheses of supported catalysts, particularly since chemical reactivities and selectivities are known to be very sensitive to the size and shape of the supported metal catalyst particles. Silica- and aluminosilica-supported chromium catalysts are used widely for the catalytic polymerization of ethylene. To examine (alkoxy)siloxy derivatives of chromium as precursors to chromium-supported catalysts, we have begun to explore synthetic routes to $\mathrm{Cr}-\mathrm{OSi}\left(\mathrm{O}^{t} \mathrm{Bu}\right)_{3}$ complexes. ${ }^{1}$ The reaction of $\mathrm{Cr}\left(\mathrm{NEt}_{2}\right)_{4}$ with $\mathrm{HOSi}\left(\mathrm{OBu}_{3}{ }_{3}\right.$ in refluxing toluene produces two isolable siloxide complexes (Eq. 1), $\mathrm{Cr}\left[\mathrm{OSi}\left(\mathrm{O}^{\mathrm{t}} \mathrm{Bu}\right)_{3}\right]_{2}\left(\mathrm{NHEt}_{2}\right)_{2}(6)$ and $\mathrm{Cr}\left[\mathrm{OSi}\left(\mathrm{O}^{\mathrm{t} B u}\right)_{3}\right]_{3}$ $\left(\mathrm{NHEt}_{2}\right)_{2}(7)$.

$\mathrm{Cr}\left(\mathrm{NEt}_{2}\right)_{4}+4 \mathrm{HOSi}\left(\mathrm{O}^{\prime} \mathrm{Bu}\right)_{3} \underset{\text { reflux }}{\stackrel{\text { toluene }}{\longrightarrow}}$<smiles>CCNC(NCC)(OCC)O[Mg]C(C)(C)C</smiles>

(6)

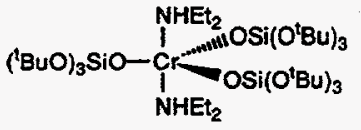

(7)
Compounds 6 and 7 are high spin and possess magnetic moments of $4.7 \mu_{\mathrm{B}}$ and $3.7 \mu_{\mathrm{B}}$, respectively. While this reaction does not provide a high yield of a single product that can be used in a convenient route to chromium silicate materials, it does offer the opportunity to closely compare analogous chromium(II) and chromium(III) siloxide structures.

1. K.W. Terry, P.K. Gantzel, and T.D. Tilley, Inorg. Chem. 32, 5402 (1993).

\section{Work in Progress}

With zirconium complexes, we are exploring in more detail the mechanism for the isobutylene elimination reactions that lead to network formation. These studies include kinetic runs, attempts to isolate and/or characterize clusters from initial condensation reactions, and development of model systems that should simplify the reaction process and make it more amenable to study. Our work with chromium-based precursors is continuing with studies to determine what solid state materials can be obtained, and with evaluations of physical and catalytic properties for the resultant products. We are also developing synthetic routes to $-\mathrm{O}_{2} \mathrm{P}\left(\mathrm{O}^{\mathrm{t}} \mathrm{Bu}\right)_{2}$ complexes, and molecular approaches to more efficient phosphors based on manganese-doped zinc orthosilicate. 


\section{Potentially Catalytic and Conducting Polyorganometallics*}

\author{
K. Peter C. Vollhardt, Investigator
}

\section{INTRODUCTION}

Polyorganometallics have great potential as catalysts for known or new organic transformations and as building blocks for novel electronic materials. Classical routes toward their assembly lack specificity and predictability, and their chemistry is complicated by dissociation into smaller fragments. This project constitutes an interdisciplinary approach to the designed construction of polymetallic arrays, anchored rigidly on novel $\pi$-ligands that enforce hitherto unprecedented metallic topologies. For this purpose, new synthetic organic methodology has been developed that allows the stepwise chemo-, regio-, and loco- (i.e., identity of the metal sequence in heterometallic systems) specific building-up of strain and/or electronically activated $\pi$-systems and attached cluster-chains. The physical and chemical properties of the resulting molecules are unparalleled and include: extreme ligand deformations, highly strained metal-metal bonds, intramolecular organic fragment migrations, new organic reactions, intrachain electron transfers, and thermally reversible photochemical storage processes.

1. Synthesis and Chemistry of Heterobimetallic Fulvalene Complexes Containing $\mathrm{W}, \mathrm{Mo}$, and $\mathrm{Rh}$ (Publication 1)

\section{A.P. Kahn,$^{\dagger}$ R. Boese, ${ }^{\ddagger} J$. Blümel,$\S$ and K.P.C. Vollhardt}

In the complexes $\mathrm{Fv}\left[\mathrm{W}(\mathrm{CO})_{3} \mathrm{Me}\right]\left[\mathrm{Rh}(\mathrm{CO})_{2}\right](1)$ and $\mathrm{Fv}\left[\mathrm{Mo}(\mathrm{CO})_{3} \mathrm{Me}\right]\left[\mathrm{Rh}(\mathrm{CO})_{2}\right](6)$ an alkylmetalcarbonyl nucleus is juxtaposed to the $\mathrm{CpRh}(\mathrm{CO})_{2}$ unit. Both, when separated, exhibit their own characteristic thermal and photochemistry. Of particular interest was to anchor these two fragments in close proximity, in order to observe potentially new synergistic chemistry. Consequently, the photo- and thermal transformations of 1 and 6 were investigated and reaction mechanisms studied by ${ }^{13} \mathrm{CO}$ labeling and low temperature argon matrix isolation experiments. The bulk transformations, including the reversible decarbonylation steps, are summarized in Figures 1-1 and 1-2. They reveal that photo-

*This work was supported by the Director, Office of Energy Research, Office of Basic Energy Sciences, Chemical Sciences Division, of the U.S. Department of Energy under Contract No. DE-AC03-76SF00098.
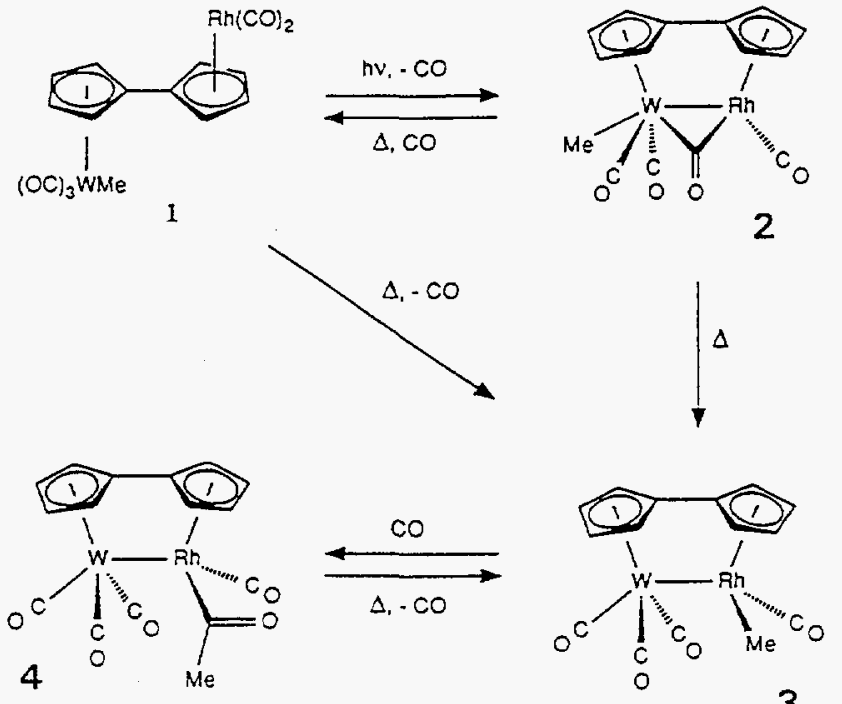

Figure 1-1. Photo- and thermal chemistry of fulvaiene complexes 1-4.

decarbonylation of 1 produces 2 , which then thermolyzes by an unusual methylshift to isomer 3 , whereas heating 1 produces 3 directly, the latter carbonylating reversibly to 4 . The role of 4 is highlighted in the direct conversion of 5 via intermediate 6 to the molybdenum analog of 4, compound 7 , which decarbonylates to the analog of 3 , complex 8 .

In order to corroborate structural assignments, the $\mathrm{x}$ ray structure of 4 was solved (Figure 1-3): $\overline{P 1}, a=$ 13.529(3) $\AA, b=15.813(3) \AA, c=16.164(3) \AA, \alpha=$ $62.72(1)^{\circ}, \beta=87.24(1)^{\circ}, \gamma=86.32(1)^{\circ}, V=3065.9(11) \AA^{3}$, $Z=8, R_{w}=0.045$. The W-Rh bond length of $2.990(1) \AA$ is

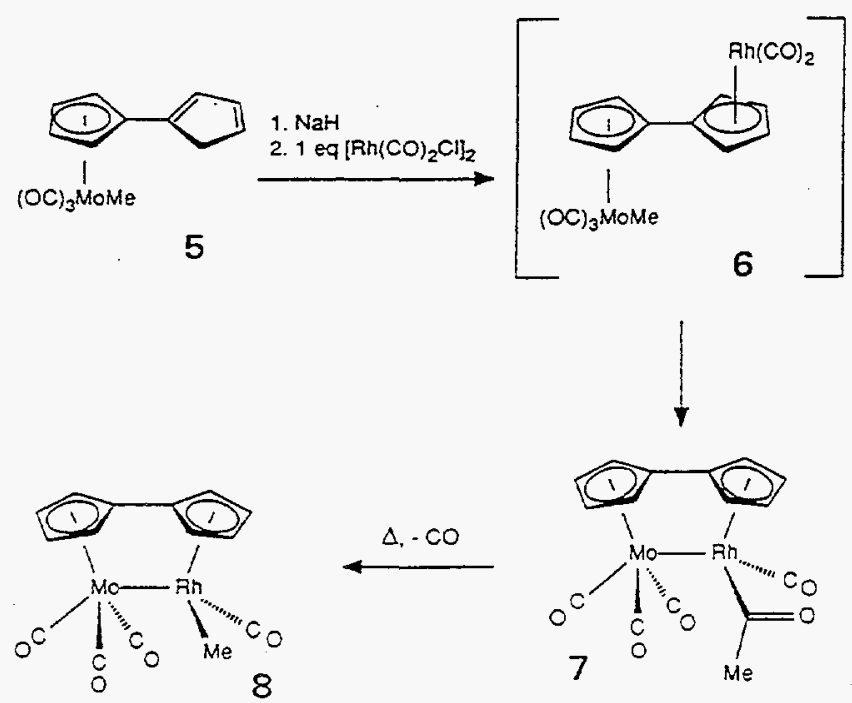

Figure 1-2. Synthesis and thermal chemistry of acyl 7. The intermediate 6 was not observed. 


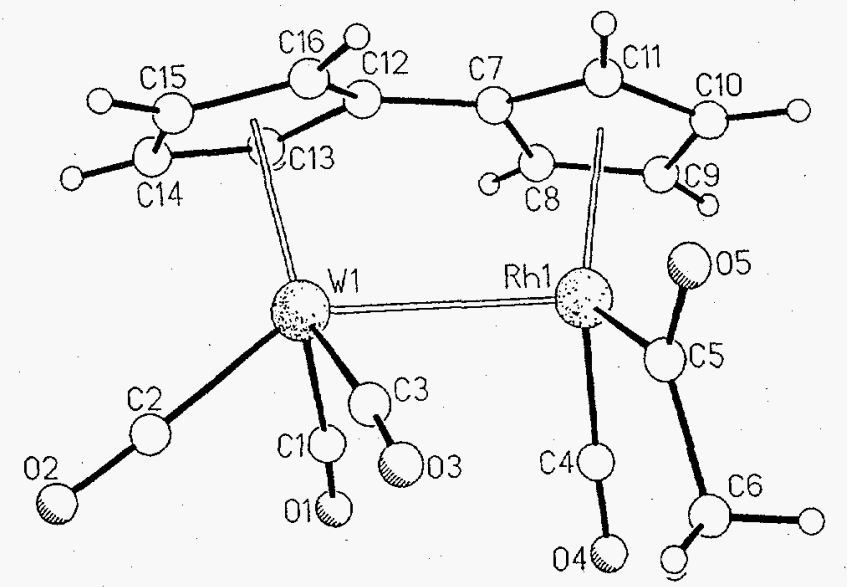

Figure 1-3. SHELXTL drawing of $\mathrm{Fv}(\mathrm{CO})_{3} \mathrm{WRh}(\mathrm{CO}) \mathrm{C}(\mathrm{O}) \mathrm{Me}$ (4).

the longest such structurally determined separation of its kind yet known, a consequence of the distortion from coplanarity of the two Cp-rings (dihedral angle $24.4^{\circ}$ ) and eclipsing of the remaining ligands.

Compound 2 exhibits fluxionality at room temperature on the NMR time scale, indicating a bridging-terminal

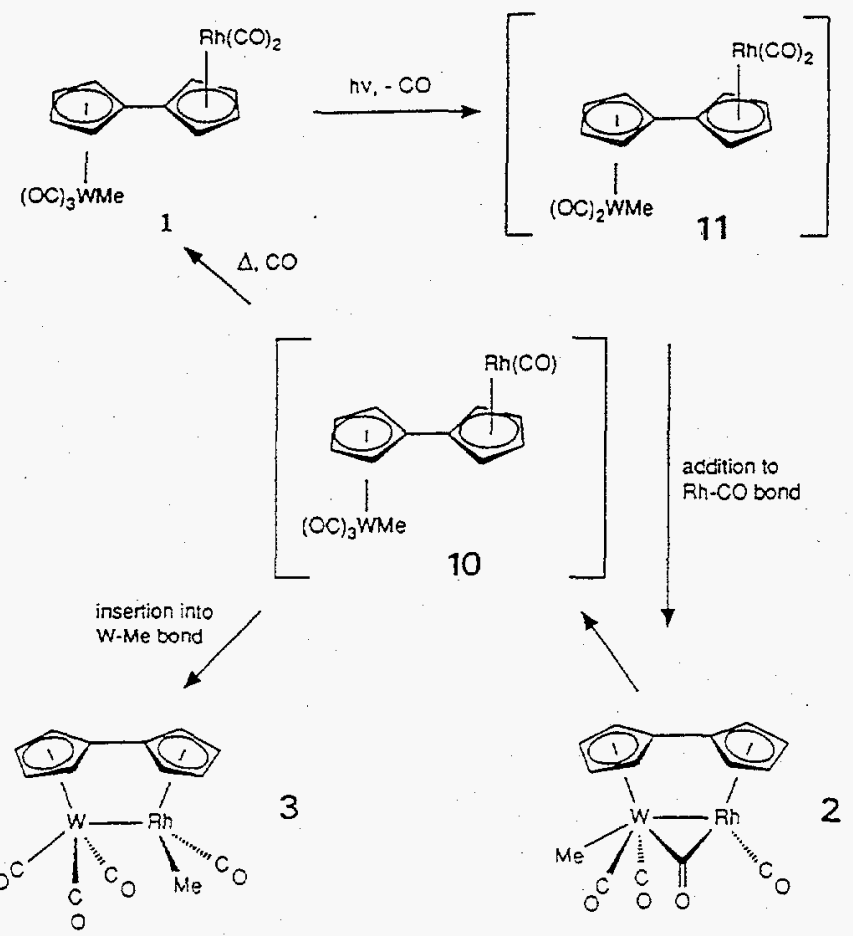

Figure 1-4. Proposed mechanism for the interconversion of 1,2 , and 3 .
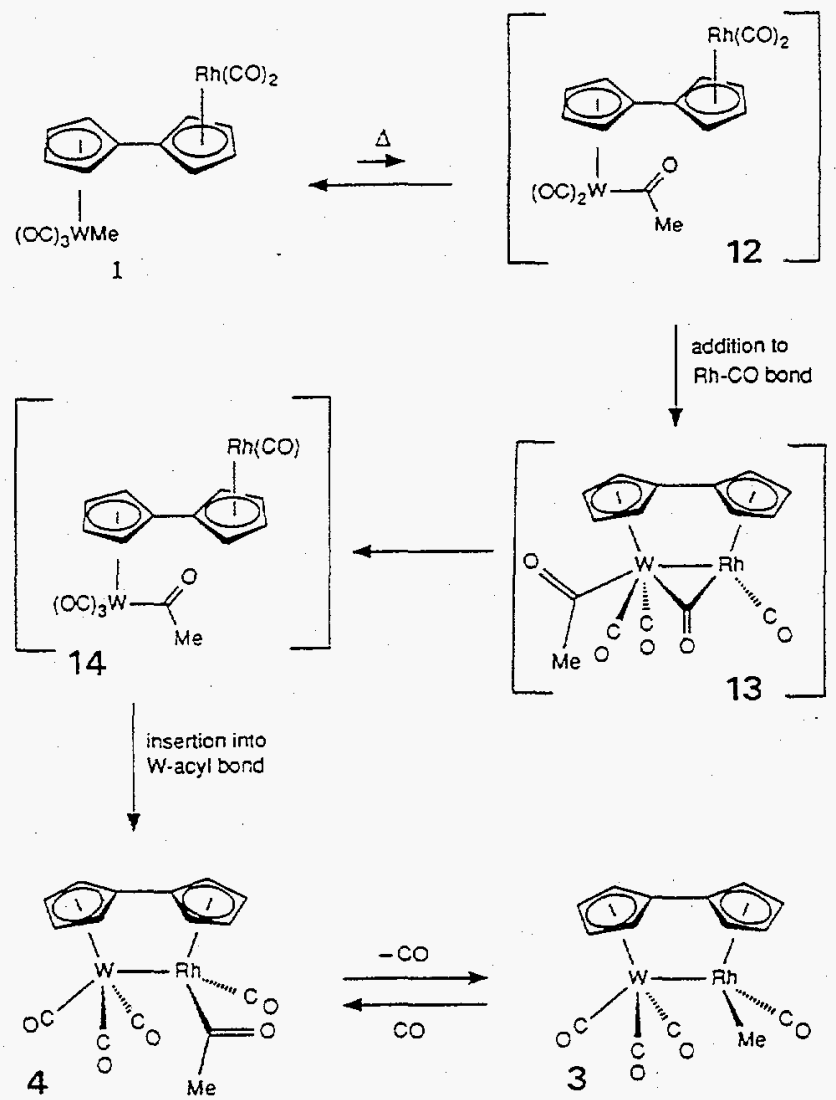

Figure 1-5. Proposed mechanism for the interconversion of 1,3 , and 4 .

carbonyl exchange involving only the Rh-CO ligands, the magnetic environment of the methyl group remaining unchanged.

The ${ }^{13} \mathrm{CO}$ labeling and matrix isolation experiments on these systems leads to the postulate of two distinct mechanisms describing the photo- and thermal chemistry of 1 (and 6). Thus, it is the W site that loses a CO ligand in the photodecarbonylation of 1 (Figure 1-4), but, in contrast, it is the Rh center that is the source of $\mathrm{CO}$ in the thermal process (Figure 1-5). Such duality of mechanism in a heterobimetallic cluster of this type is unprecedented.

\footnotetext{
Permanent address: ARCO Chemicals, New Town Square, PA 19073 , USA.

¥Permanent address: Institut für Anorganische Chemie, Universität-GH Essen, Universitätsstr. 5-7, D-45117 Essen, Germany.

Permanent address: Anorganisch-Chemisches Institut der TU München, Lichtenbergstr. 4, 85747 Garching, Germany.
} 
2. Electron-Reservoir Complexes $\left[\mathrm{Fe}^{\mathrm{I}} \mathrm{Cp}\right.$ (arene)] as Selective Initiators for a Novel Electron Transfer Chain Catalyzed Reaction: Generai Synthesis of Fulvalene-Bridged Homo- and Heterodinuclear Zwitterions (Publication 2)

\section{D.S. Brown, M.-H. Delville-Desbois, ${ }^{\dagger} R$. Boese, ${ }^{\ddagger}$ K.P.C. Vollhardt, and D. Astruc ${ }^{\dagger}$}

Many non-redox reactions proceed by the electrontrapsfer chain (ETC) mechanism. We have now observed ETC catalysis of a redox reaction, the intramolecular disproportionation of dinuclear fulvalene carbonyl complexes in the presence of $\mathrm{PR}_{3}(\mathrm{R}=\mathrm{Me}, \mathrm{OMe})$. The result is the cleavage of the metal-metal bond, replacement of one $\mathrm{CO}$ by two $\mathrm{PR}_{3}$ ligands at a single metal center, and intramolecular electron transfer (Figure 2-1; $\mathrm{R}^{\prime}=\mathrm{H}$, Me in the $\mathrm{Fe}$ catalyst). The starting materials and products are listed in Table 2-1. The catalysts, electron-reservoir complexes $\left[\mathrm{Fe}^{\mathrm{I}} \mathrm{Cp}\left(\mathrm{C}_{6} \mathrm{R}_{6}\right)\right]$, are the most electron-rich, stable neutral compounds known (based on ionization potential values), and are ideal mediators allowing reduction at very negative potentials $\left(E^{\circ}=-1.3\right.$ to $-1.9 \mathrm{~V}$ vs. SCE). They can be used in precise amounts, and their redox potentials $E^{\circ}$ FelFell can be adjusted by the choice of the ring substituents, which provides selectivity.

The molecular structure of the zwitterions was proven by elemental analysis, high resolution ${ }^{1} \mathrm{H},{ }^{13} \mathrm{C}$, and ${ }^{31} \mathrm{P}$ NMR spectroscopy, and mass spectrometry. In the case of entry 4 (Table 2-1), the $x$-ray crystal structure of the product shows the anti conformation of the two metal complex fragments (Figure 2-2), which is most probably common to all the zwitterions reported here. Cyclic voltammetry was used to monitor the ETC-catalyzed process by following the disappearance of the starting complexes and appearance of zwitterions.

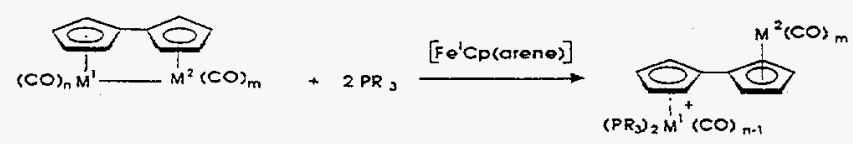

Figure 2-1. Electron-transfer-chain catalyzed zwitterion synthesis.
Table 2-1. Summary of Synthetic Results.

\begin{tabular}{cllllll}
\hline Entry & $\mathrm{M}^{1}$ & $\mathrm{M}^{2}$ & $n$ & $m$ & $\mathrm{R}$ & $\begin{array}{c}\text { Yield } \\
(\%)\end{array}$ \\
\hline 1 & $\mathrm{Mo}$ & $\mathrm{Mo}$ & 3 & 3 & $\mathrm{Me}$ & 60 \\
2 & $\mathrm{~W}$ & $\mathrm{~W}$ & 3 & 3 & $\mathrm{Me}$ & 70 \\
3 & $\mathrm{Fe}$ & $\mathrm{W}$ & 2 & 3 & $\mathrm{OMe}$ & 75 \\
4 & $\mathrm{Fe}$ & $\mathrm{W}$ & 2 & 3 & $\mathrm{Me}$ & 79 \\
5 & $\mathrm{Ru}$ & $\mathrm{Mo}$ & 2 & 3 & $\mathrm{Me}$ & 80 \\
6 & $\mathrm{Ru}$ & W & 2 & 3 & $\mathrm{Me}$ & 83 \\
\hline
\end{tabular}

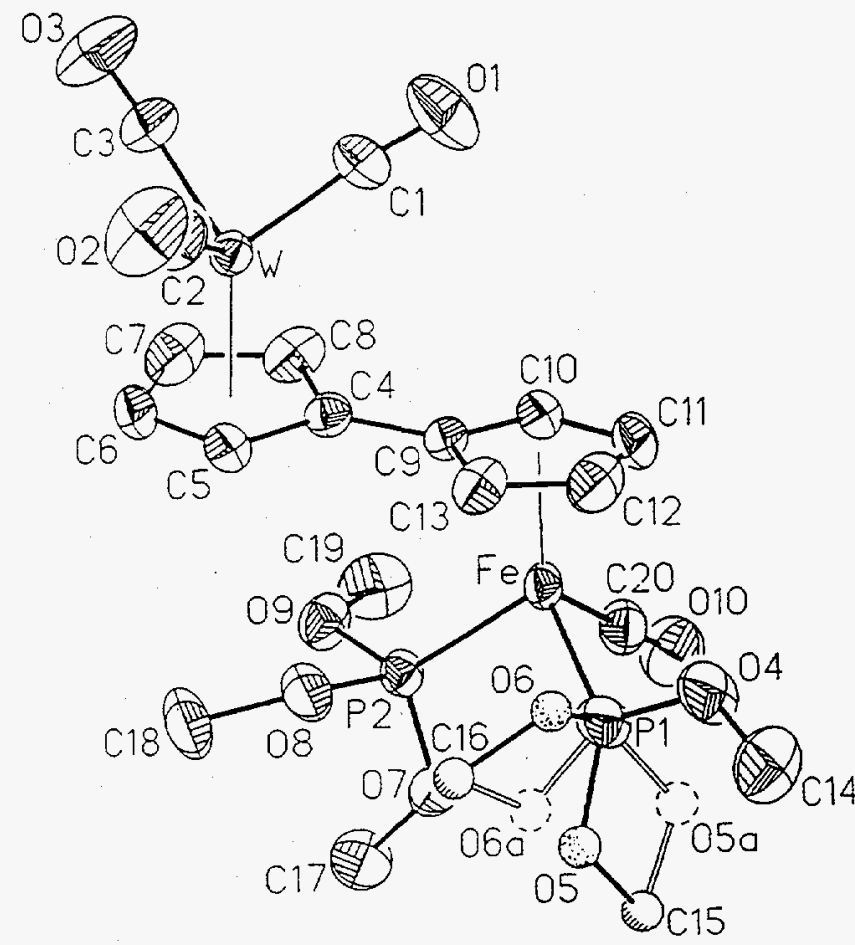

Figure 2-2. ORTEP drawing of Fv[W(CO) $\left.\left.{ }_{3}^{-}\right]\left[\mathrm{Fe}(\mathrm{CO}) \mathrm{PMe}_{3}\right)_{2}{ }^{+}\right]$: $P \overline{1}, a=9.766$ (1) Å, $b=10.973$ (1) $\AA, c=12.536$ (2) $\AA, \alpha=77.60$ (1) , $\beta=73.43(1)^{\circ}, \gamma=81.13(1)^{\circ}, V=1242.5(3) \AA^{3}, Z=2, R_{w}=0.0426$.

\footnotetext{
${ }_{\dagger}^{\dagger}$ Permanent address: Laboratoire de Chimie Organique et Organométallique, URA CNRS $n^{\circ} 35$, Université Bordeaux I, 351 Cours de la Libération, 33405 Talence Cédex, France.

$\doteqdot$ Permanent address: Institut für Anorganische Chemie, Universität-GH Essen, Universitätsstr. 5-7, D-45117 Essen, Germany.
} 
3. Chemistry of Dinuclear Fulvalene Complexes: Dihydrides, Zwitterions, and Ring-Slippage Complexes Derived from $\mathrm{FvM}_{2}(\mathrm{CO})_{6}(\mathrm{M}=\mathrm{Mo}, \mathrm{W})$ (Publication 3)

\section{Tilset, ${ }^{\dagger}$ K.P.C. Vollhardt, and R. Boese $e^{\ddagger}$}

Reduction of the metal-metal-bonded complex $\mathrm{FvM}_{2}(\mathrm{CO})_{6}$ generated the dianion $\mathrm{FvM}_{2}(\mathrm{CO})_{6}{ }^{2-}$. An X-ray crystallographic analysis of $\left[\mathrm{Et}_{4} \mathrm{~N}^{+}\right]_{2}\left[\mathrm{FvW}_{2}(\mathrm{CO})_{6}\right]^{2-}$ (monoclinic space group $P 2{ }_{1} / c, a=7.687(2) \AA, b=$ 13.752(4) (̊), $c=16.297(5) \AA, \beta=94.80(2)^{\circ}, V=$ $1716.8(8) \AA^{3}, Z=4$ ) showed the dianion to contain a planar Fv ring system bonded to the two metal centers in an anti fashion. The dianion reacted with a number of electrophiles to yield the neutral species $\mathrm{FvW}_{2}(\mathrm{CO})_{6} \mathrm{E}_{2}(\mathrm{E}=\mathrm{H}$, Me, Et, $\sigma-\mathrm{C}_{3} \mathrm{H}_{5}, \mathrm{CH}_{2} \mathrm{Ph}$ ). The $\mathrm{p} K_{\mathrm{a}}$ values for the two consecutive deprotonations of $\mathrm{FvW}_{2}(\mathrm{CO})_{6} \mathrm{H}_{2}$ were determined as 14.0 and 16.6 by equilibrium measurements in acetonitrile. Thermolysis and photolysis of $\mathrm{FvW}_{2}(\mathrm{CO})_{6} \mathrm{H}_{2}$ yielded $\mathrm{FvW}_{2}(\mathrm{CO})_{6}$ and $\mathrm{H}_{2}$. Unlike $\mathrm{Cp}_{2} \mathrm{~W}_{2}(\mathrm{CO})_{6}, \mathrm{FvW}_{2}(\mathrm{CO})_{6}$ underwent protonation at the $\mathrm{W}-\mathrm{W}$ bond by $\mathrm{HBF}_{4} \cdot \mathrm{Et}_{2} \mathrm{O}$ in acetonitrile. Reactions of $\mathrm{FvW}_{2}(\mathrm{CO})_{6}$ and $\mathrm{FvMo}_{2}(\mathrm{CO})_{6}$ with $\mathrm{PMe}_{3}$ and $\mathrm{Me}_{2} \mathrm{PCH}_{2} \mathrm{PMe}_{2}$ (dmpm) resulted in generation of the dinuclear zwitterions $\mathrm{FvM}_{2}(\mathrm{CO})_{5}\left(\mathrm{PMe}_{3}\right)_{2}$ and $\left.\mathrm{FvM}_{2}(\mathrm{CO})_{5}\right)(\mathrm{dmpm})$, respectively. An x-ray crystallographic analysis of $\mathrm{FvMO}_{2}(\mathrm{CO})_{5}$ (orthorhombic space group $P 22_{1} 2_{1} 2_{1}, a=9.1049(8) \AA, b=12.2598(14) \AA, c=$ $\left.20.1606(18) \AA, V=2250.4(7) \AA^{3}, Z=4\right)$ showed an anti coordination of the $\mathrm{Mo}(\mathrm{CO})_{3}{ }^{-}$and $\mathrm{Mo}(\mathrm{CO})_{2}(\mathrm{dmpm})^{+}$ moieties at a planar $\mathrm{Fv}$ ligand. Electrophiles added at the anionic part of the zwitterions, whereas $\mathrm{LiAlH}_{4}$ effected reduction of coordinated $\mathrm{CO}$ to $\mathrm{CH}_{3}$ at the cationic center of $\mathrm{FvMo}_{2}(\mathrm{CO})_{5}\left(\mathrm{PMe}_{3}\right)_{2}$. Excess $\mathrm{PMe}_{3}$ caused the conversion of $\mathrm{FvMo}_{2}(\mathrm{CO})_{5}\left(\mathrm{PMe}_{3}\right)_{2}$ and $\mathrm{FvMO}_{2}(\mathrm{CO})_{5}-(\mathrm{dmpm})$ to $\mathrm{Mo}(\mathrm{CO})_{3}\left(\mathrm{PMe}_{3}\right)_{3}$ along with $\mathrm{FvMo}(\mathrm{CO})_{2}\left(\mathrm{PMe}_{3}\right)_{2}$ and FvMo $(\mathrm{CO})_{2}$ (dmpm), respectively. These reactions constitute the first ring-slippage reactions that have been observed in fulvalene metal complexes. When treated with $\mathrm{Mo}(\mathrm{CO})_{3}(\mathrm{NCMe})_{3}, \quad \mathrm{FvMo}(\mathrm{CO})_{2}\left(\mathrm{PMe}_{3}\right)_{2}$ cleanly regenerated $\mathrm{FvMO}_{2}(\mathrm{CO})_{5}\left(\mathrm{PMe}_{3}\right)_{2}$.

\footnotetext{
Termanent address: Department of Chemistry, University of Oslo, P.O. Box 1033 Blindern, N-0315 Oslo, Norway.

¥Permanent address: Institut für Anorganische Chemie, Universität-GH Essen, Universitätsstr. 5-7, D-45117 Essen, Germany.
}

4. Decasubstituted Decaphenylmetallocenes (Publication 4)

\section{R. H. Lowack and K.P.C. Vollhardt}

Cyclopentadienyl ligands with bulky substituents, such as t-butyl or phenyl, can form complexes that differ drastically from those containing the unsubstituted parent ligands. Their steric environment can stabilize the complex kinetically by shielding the reactive metal center and the cyclopentadienyl ring. Perphenylated cyclopentadienyl systems are especially well stabilized, even in the case of "unusual" oxidation states. This is due mainly to the stereorigidity of these systems and probably to a lesser extent to charge delocalization into the phenyl groups. For example, in decaphenylstannocene the rotation of the ten phenyl rings and of the two $\mathrm{Cp}$-units is severely restricted by steric crowding. The planar cyclopentadienyl rings are forced into a parallel orientation, and attack at the tin atom is obstructed. Thus, the $S_{10}$-symmetrical decaphenylstannocene does not decompose up to $350^{\circ} \mathrm{C}$ and can be stored "indefinitely" in wet air. In contrast, the very airand water-sensitive unsubstituted stannocene, and even the decamethyl, decabenzyl, or octaphenyl derivatives, are markedly less stable.

The other decaphenylmetallocenes that have been synthesized and satisfactorily characterized, those with $\mathrm{Ce}$, $\mathrm{Sn}, \mathrm{Pb}, \mathrm{Ni}$, or Mo as the metal atom, show similarly higher stability than the corresponding unsubstituted metallocenes. On the other hand, all the decaphenylmetallocenes and the free ligand itself are reported to be almost insoluble in all common organic solvents. This severe disadvantage of the pentaphenylcyclopentadienyl ligand is due to the compact shape, which facilitates crystallization, and to protection of the metal center from interactions with the solvent. We report here that 1,2,3,4,5-pentaphenylcyclopentadiene (2), derived from 2,3,4,5-pentaphenylcyclopentadienone (1), can be selectively para-acetylated under Friedel-Crafts conditions to 3 , the source of a number of novel materials (Figure 4-1). Compound 3 is remarkably acidic, being deprotonated by amine bases to the symmetrical and quite unreactive anion. Acetalization to $\mathbf{6}$, followed by deprotonation to 7 and metallation, produced to first soluble, derivatized decaphenylstannocene and -plumbocene, 8 and 9 , respectively. Selective hydrolysis back to 6 was possible with hydrazinium tosylate. One-electron oxidation of 7 with $\mathrm{Ag}^{+}$furnished the blue-violet radical 10, a promising species on route to transition metal derivatives. 


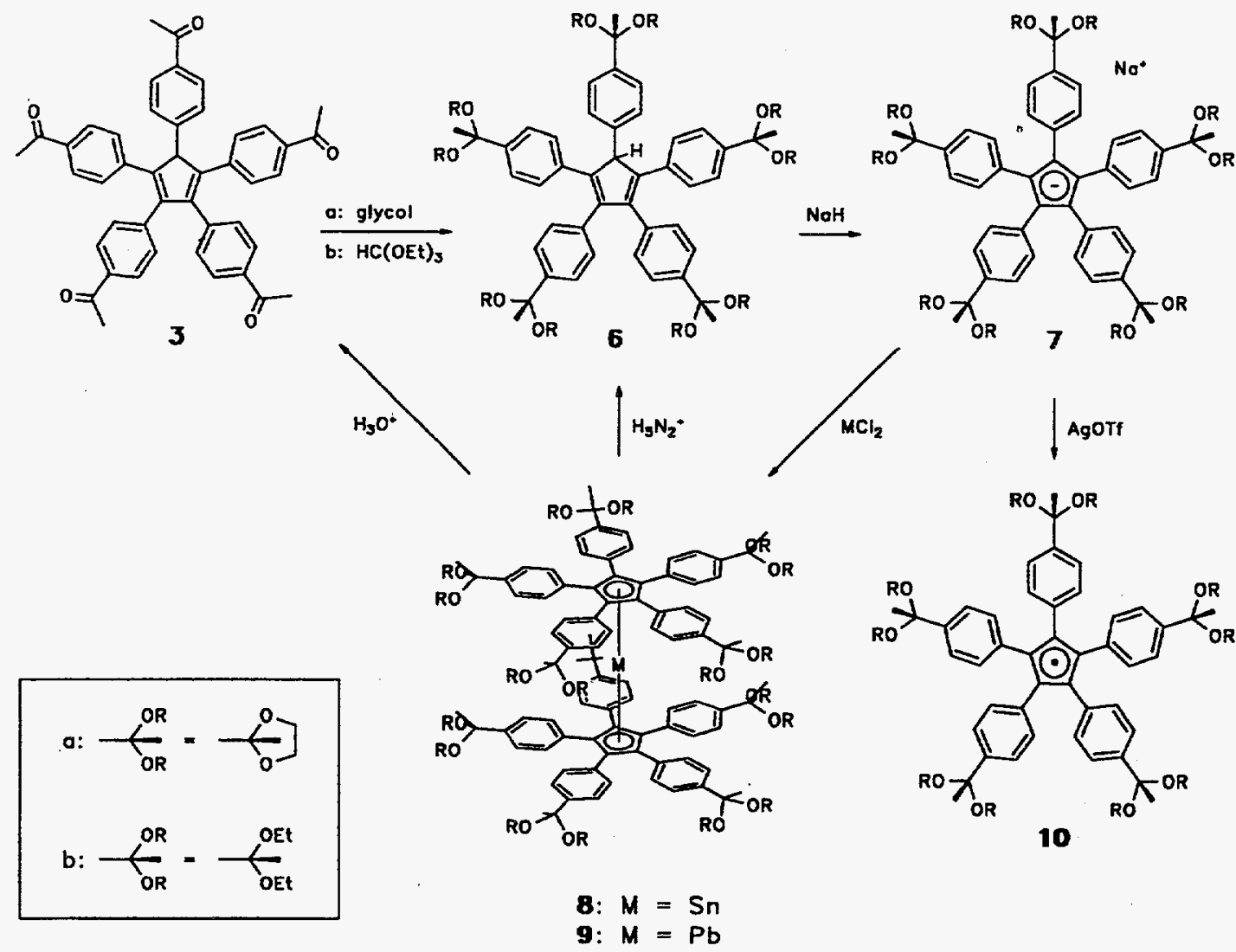

Figure 4-1. The synthesis of functionalized decaphenylmetallocenes.

\section{FY 1994 PUBLICATIONS AND REPORTS}

\section{Refereed Journals}

1. A.P. Kahn, R. Boese, J. Blümel, and K.P.C. Vollhardt, "Synthesis and Chemistry of Heterobimetallic Fulvalene Complexes Containing W, Mo, and Rh," J. Organomet. Chem. 472, 149 (1994); LBL-36652.

2 D.S. Brown, M.-H. Delville-Desbois, R. Boese, K.P.C. Vollhardt, and D. Astruc, "Electron-Reservoir Complexes $\left(\mathrm{Fe}^{\mathrm{I}} \mathrm{Cp}\right.$ (arene)] as Selective Initiators for a Novel ElectronTransfer-Chain Catalyzed Reaction: General Synthesis of Fulvalene-Bridged Homo- and Heterodinuclear Zwitterions," Angew. Chem. 106, 715 (1994); Angew. Chem., Int. Ed. Engl. 33, 661 (1994); LBL-36653.

3. M. Tilset, K.P.C. Vollhardt, and R. Boese, "Chemistry of Dinuclear Fulvalene Complexes: Dihydrides, Zwitterions, and Ring-Slippage Complexes Derived from $\mathrm{FvM}_{2}(\mathrm{CO})_{6}$ $(\mathrm{M}=\mathrm{Mo}, \mathrm{W})$," Organometallics 13, 3146 (1994); LBL36654.

4. R.H. Lowack and K.P.C. Vollhardt, "Decasubstituted Decaphenylmetallocenes," J. Organomet. Chem. 476, 25 (1994); LBL-36366.

5. J.A. King Jr. and K.P.C. Vollhardt, "Thermally and Photochemically Induced Vinyl-hydrogen Activation of
[ $\eta^{4}-1,2: 3,4$ ( trans-1,3,5-hexatriene) $]\left(\eta^{5}\right.$-cyclopentadienyl) cobalt: Regio- and Stereospecific Hydrogen Migrations," J. Organomet. Chem. 470, 207 (1994); LBL-35895.

6. R. Boese, D. Bläser, W.E. Billups, M.M. Haley, A.H. Maulitz, D.L. Mohler, K.P.C. Vollhardt, "The Effect of Fusion of Angular Strained Rings on Benzene: Crystal Structures of 1,2-Dihydrocyclobuta[ $a]$ cyciopropa[ $c]$-, 1,2,3,4-Tetrahydrodicyclobuta[ $a, c]$-, 1,2,3,4-Tetrahydrodicyclobuta[ $a, c]$ cyclopropa[ $e]-$, and $1,2,3,4,5,6$-Hexahydrotricyclobuta[a,c,e]benzene," Angew. Chem. 106, 321 (1994); Angew. Chem., Int. Ed. Engl. 33, 313 (1994); LBL35894.

\section{Invited Talks}

7. K.P.C. Vollhardt, "The Cobalt Way to Total Synthesis," Randolph T. Majors Lecturer, University of Connecticut, Storrs, CT, October 25, 1993.

8. K.P.C. Vollhardt, "The Phenylenes. A Testing Ground for the Origin of Delocalization in Benzene," Randolph T. Majors Lecturer, University of Connecticut, Storrs, CT, October 26, 1993.

9. K.P.C. Vollhardt, "Oligocyclopentadienylmetals: Remarkable Organometallic Materials," Randolph T. Majors Lecturer, University of Connecticut, Storrs, CT, October 27, 1993. 
10. K.P.C. Vollhardt, "Recent Breakthroughs in CobaltMediated Alkyne Cyclizations," Plenary Lecture, Finnish Chemical Congress, Symposium on Synthetic Chemistry, Helsinki, Finland, November 4, 1993.

11. K.P.C. Vollhardt, "Cobalt-Mediated Total Synthesis of Natural and Unnatural Products," University of Oslo, Norway, November 5, 1993.

12. K.P.C. Vollhardt, "Cobalt-Mediated Total Synthesis of Natural and Unnatural Products," University of Oregon, Eugene, OR, December 3, 1993.

13. K.P.C. Vollhardt, "The Phenylenes," Plenary Lecture, 3rd Tohwa International Symposium on Synthetic and
Mechanistic Hydrocarbon Chemistry, Fukuoka, Japan, December 6-9, 1993.

14. K.P.C. Vollhardt, "Oligocyclopentadienylmetals: Remarkable Organometallic Materials," Kangweon National University, Chuncheon, South Korea, April 21, 1994.

15. K.P.C. Vollhardt, "Cobalt-Mediated Total Synthesis of Natural and Unnatural Products," 73rd Annual Meeting of the Korean Chemical Society, Inchon, South Korea, April 22, 1994. 


\section{HEAVY-ELEMENT CHEMISTRY}

\section{Actinide Chemistry*}

\author{
Norman M. Edelstein, Richard A. Andersen, Kenneth N. \\ Raymond, and David K. Shuh, Investigators
}

\section{General Introduction}

Development of new technological processes for the use, safe handling, storage, and disposal of actinide materials relies on the further understanding of basic actinide chemistry and the availability of a cadre of trained personnel. This research program is a comprehensive, multifaceted approach to the exploration of actinide chemistry and to the training of students. Research efforts include synthetic organic and inorganic chemistry for the development of new chemical agents and materials, chemical and physical elucidation through various characterization techniques, and thermodynamic and kinetic studies for the evaluation of complex formation.

The development and understanding of complexing agents that specifically and effectively sequester actinide ions is one program aspect. Such agents are intended for the decorporation of actinides in humans, in the environment, and in systems related to the nuclear fuel cycle. Extensive studies are under way to prepare organometallic and coordination compounds of the f-block elements that show the differences and similarities among the f-elements, and between the f- and d-transition series elements. Optical and magnetic studies on actinides as isolated ions in ionic solids, and in molecules, provide information about electronic properties as a function of atomic number.

\section{Specific Sequestering Agents for the Actinides}

\author{
Kenneth N. Raymond, Investigator
}

\section{INTRODUCTION}

The fundamental coordination chemistry of the actinides in their various oxidation states provides the basis

*This work was supported by the Director, Office of Energy Research, Office of Basic Energy Sciences, Chemical Sciences Division, of the U.S. Department of Energy under Contract No. DE-AC03-76SF00098. for the development of new technologies for the separation and isolation of these elements. Agents that selectively and strongly bind f-transition elements are of particular technological importance in current efforts for the remediation of nuclear waste sites. However, this chemistry also has applications in other fields. The lanthanide elements ( $4 \mathrm{f}$ series) provide good models for the trivalent actinides (5f series); however, there are independent strong interest in the coordination chemistry of the lanthanides because of the use of gadolinum complexes as magnetic resonance imaging (MRI) enhancement agents. Several of our current chelating agents are likely to be used as human decorporation agents in the case of actinide contamination. Recent experiments have shown, for the first time, ligands that are effective in the decorporation of uranyl and americium(III) from test animals.

\section{Measuring Trace Uranium (Publication 2)}

\section{R. Cox, D.A. Buttry, P. Bonnesen, K.N. Raymond}

The sensitivity of a quartz crystal microbalance has been combined with a high surface area support for the binding of uranyl ion via specific ligand groups. Piezoelectric quartz crystals have specific normal modes of vibration, several in the megahertz region. These have very narrow bandwidths so that shifts of a few hertz can be measured. Mass changes on the surface of the crystal then cause a measurable change in frequency. By attaching a uranyl-specific ligand to the quartz crystal surface, reversible binding of uranyl ion has been measured by a frequency change $(290 \mathrm{~Hz})$ in response to a 10 micromolar uranyl ion solution and resultant binding of the uranyl ion. This binding is shown to be relatively specific with respect to the metal cation.

2. In vivo Chelation of $\mathrm{Am}(\mathrm{III}), \mathrm{Pu}(\mathrm{IV}), \mathrm{Np}(\mathrm{V})$ and U(VI) in Mice by TREN-(Me-3,2-HOPO)

(Publication 1)

\section{P.W. Durbin, B. Kullgren, J. Xu, and K.N. Raymond}

Octadentate 3,4,3-Li(1,2-HOPO), composed of the acidic hydroxypyridine isomer, $1,2-\mathrm{HOPO}$, is the most effective ligand yet prepared for in vivo chelation of $\mathrm{Pu}(\mathrm{IV})$, and $\mathrm{Am}(\mathrm{III})$, but it is difficult to prepare and is acutely toxic at high dosage. Hexadentate TREN-(Me-3,2HOPO), composed of the less acidic Me-3,2-HOPO isomer, can be produced in relatively large quantities (see 
<smiles>CC(=O)c1ccn(C)c(=O)c1O</smiles>

$\mathrm{Me}-3,2-\mathrm{HOPO}$

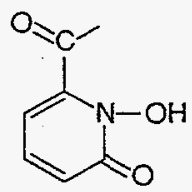

$1,2-\mathrm{HOPO}$

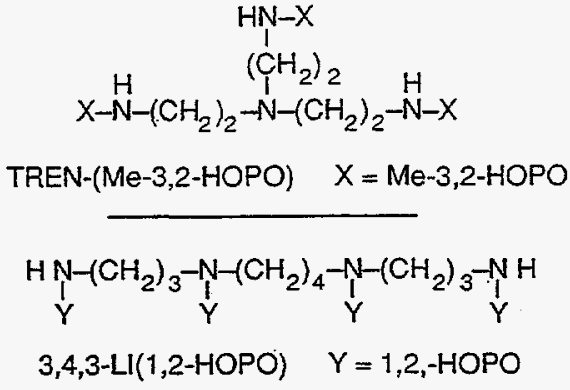

Figure 2-1. Molecular structure of me-3,2-HOPO and 1,2-HOPO hydroxypyridinone isomers, TREN-(Me-3,2-HOPO, and 3,4,3-LI(1,2HOPO). (XBD 951-120)

Figure 2-1). Tren-(Me-3,2-HOPO) $\left(30 \mu\right.$ mol. $\mathrm{kg}^{-1}$ injected intraperitoneally in mice) removed significant body $\mathrm{Pu}(\mathrm{IV})$, $\mathrm{Am}(\mathrm{III}), \mathrm{Np}(\mathrm{V})$ (compared with controls), and those actinide reductions were significantly greater than were obtained with $\mathrm{CaNa}_{3}$-DTPA. TREN-(Me-3,2-HOPO) was almost as effective for reducing body $\mathrm{Pu}(\mathrm{IV})$ as $3,4,3$ LI(1,2-HOPO). TREN-(Me-3,2-HOPO) is of low acute toxicity in mice and its clinical potential merits further investigation.

3. Solution Structure and Dynamics of Lanthanide Complexes of the Macrocyclic Polyamino Carboxylate DTPA-dien. NMR Study and Crystal Structures of the Lanthanum(III) and Europium(III) Complexes (Publication 3)

\section{S.J. Franklin and K.N. Raymond}

An 18-membered macrocyclic DTPA-bisamide ligand (DTPA = diethylenetriamine petaacetic acid) containing a heteroatom in the amide link has been prepared via the condensation of DTPA-dianhydride and diethylenetriamine. The solution structures of the two isomeric pairs present in the Ln(III) complexes of DTPA-dien have been investigated by ${ }^{1} \mathrm{H}$ NMR. One enantiomeric pair exhibits slow exchange on the NMR timescale at low temperatures $\left(0-25^{\circ} \mathrm{C}\right)$ and "dynamic" behavior at higher temperatures.
The other isomeric pair exhibits an unusual "static" behavior; exchange remains slow even at $95^{\circ} \mathrm{C}$. Peak assignments for the Eu DTPA-dien spectra are given based on deuteration studies, 2D COSY spectroscopy, and 2D EXSY spectroscopy. 2D EXSY spectroscopy at several temperatures and mixing times showed that $\Delta \mathrm{G}^{\ddagger}{ }_{299}$ for the "dynamic" isomerization is $57.5 \pm 0.3 \mathrm{~kJ} / \mathrm{mol}$, and that the "dynamic" isomer is an intermediate for the "static" isomerization, which occurs with a change in backbone amine chirality. The structures of the lanthanum (III) and europium (III) DTPA-dien complexes have been determined by $\mathrm{x}$-ray analysis. [La DTPA-dien $\left.\mathrm{H}^{+} \mathrm{H}_{2} \mathrm{O}\right]_{2}$ $\left(\mathrm{CF}_{3} \mathrm{SO}_{3}{ }^{-}\right)_{2} \cdot 18 \mathrm{H}_{2} \mathrm{O}$ crystallizes as a carboxylate-bridged dimer in which each lanthanum ion is 11-coordinate with octadentate ligand coordination, an $\mathrm{h}^{2}$ bridging carboxylate, and one water. [Eu DTPA-dien $\left.\mathrm{H}^{+}\right]_{4}\left(\mathrm{CF}_{3} \mathrm{~S} \mathrm{O}_{3}^{-}\right)_{4} \cdot 6$ $\mathrm{NaCF}_{3} \mathrm{SO}_{3} \cdot 20 \mathrm{H}_{2} \mathrm{O}$ crystallized as a carboxylate-bridged tetramer with two crystallographically independent $\mathrm{Eu}(\mathrm{III})$ positions in which the coordination geometries about Eu1 and Eu2 are nearly identical and are described as a ninecoordinate tricapped trigonal prism with octadentate ligand coordination plus an $\mathrm{h}^{\mathbf{l}}$ bridging carboxylate. The tendency to oligomerize is attributed to the constraints imposed by the macrocycle and the hydrogen bonding available with the link heteroatom. The solution structure of the "dynamic" isomer is found to be the same as the monomer unit of the crystal structures, and the "static" isomer is similar, save a change in one terminal backbone nitrogens' chirality. These model the behavior of several MRI agents.

4. Amide Functional Group Contribution to the Stability of Gadolinium(III) Complexes in DTPA Derivatives

\section{Paul-Roth and K.N. Raymond}

The $\mathrm{Gd}^{3+}$ complexes of diethylenetriaminepentaacetic acid (DTPA) and several closely related ligands are in use, or being developed, as contrast agents for MRI. The bisamide derivatives of DTPA are, like the parent DTPA ligand, octadentate complexing agents of gadolinium, replacing two carboxylate coordinating groups with coordinating amide oxygens. Remarkably, this maintains a significant portion of the stability of the gadolinium complex and an increase in the relative selectivity for $\mathrm{Gd}^{3+}$ vs. $\mathrm{Ca}^{2+}$. The magnitude of the contribution of the amide functional groups to this stability has been investigated. A diethylenetriaminetricarboxylic acid bis-amide derivative in which the two terminal amide groups are replaced by methyl groups (bismethyldiethylenetriaminetriacetic acid, DTTA-BM) is found to have a metal ion affinity at $\mathrm{pH} 7.4$ that is $6.75 \mathrm{log}$ units smaller than the effective stability of the bis-amide DTPA derivatives, corresponding to an 
average contribution of $3.38 \mathrm{log}$ units to the stability of the complex from each amide functional group. The effective stability of the DTTA-BM Ca ${ }^{2+}$ complex relative to the bisamide ligands does not change significantly. Hence the amide functional groups contribute significant stability for gadolinium complexation but little or no enhancement of calcium complexation, explaining the relative selectivity of bis-amide ligands as gadolinium(III) complexing agents.

\section{Work in Progress}

Synthesis and Initial Biological Evaluation for in vivo Chelation of Pu(IV) by Octadentate Ligands Containing 2,3-dihydroxybenzamide and 2,3dihydroxyterephthalamide Binding Units

\section{J. Xu, P.W. Durbin, B. Kullgren and K.N. Raymond}

The linear octadentate ligand 3,4,3-LICAMC is one of the most effective chelating agents for $\mathrm{Pu}(\mathrm{IV})$ that is not acutely toxic. However, at physiological $\mathrm{pH}$, due to the weak acidity of the catechol hydroxy groups and the large proton dependence of the complexation reaction, only three of the four catecholate subunits are coordinated to $\mathrm{Pu}(\mathrm{IV})$. To overcome this disadvantage, a new topological class of octadentate ligands, H-TAM based on an "H-shaped" tetrapodal amine backbone and 2,3dihydroxyterephthalamide (TAM) binding units, has been designed and synthesized both in open and closed (macrotricyclic) architectures. With higher acidity and greater predisposition towards binding, the H-TAM ligands were expected to be stronger $\mathrm{Pu}(\mathrm{IV})$ chelating agents than 3,4,3-LICAM(C). In order to optimize the design of the HTAM chelating agents, other octadentate ligands such as 3,4,3-LIMeTAM, DFO-MeTAM, Oxo-H-TAM, and $\mathrm{H}(\mathrm{n}, 2)$-CAM(C) were also designed, synthesized, and tested for Pu(IV) chelation in vivo. When injected new ligands reduced body, liver, and skeleton Pu significantly below control levels. Soft tissue and kidney Pu were also reduced below control values by 3,4,3-LIMeTAM, DFOMeTAM, Fe(III)-DFO-MeTAM, and macrocyclic BH(2,2)$\mathrm{CAM}$, indicating their $\mathrm{Pu}(\mathrm{IV})$ complexes are more stable than Pu(IV)-3,4,3-LICAM(C) at the reduced $\mathrm{pH}$ of the kidneys. DFO-MeTAM, 3,4,3-LIMeTAM, H(2,2)MeTAM, H(2,2)-EtTAM reduced liver Pu significantly more than $\mathrm{CaNa}_{3}$-DTPA. However, kidney $\mathrm{Pu}$ after treatment with any except the first four ligands mentioned above was 1.5 to 5 times greater than the control value, indicating instability of their $\mathrm{Pu}(\mathrm{IV})$ complexes at reduced $\mathrm{pH}$. All ten ligands administered orally significantly reduced body $\mathrm{Pu}$ below the control level, significant reductions of skeletal $\mathrm{Pu}$ were obtained with 3,4,3LIMeTAM, Oxo-H(2,2)-MeTAM, and DFO-MeTAM. Compared with $\mathrm{CaNa}_{3}-\mathrm{DTPA}$, significantly greater reductions of skeleton $\mathrm{Pu}$ were obtained with oral $\mathrm{H}(2,2)$ CAM(C) and DFO-MeTAM, and significantly greater reductions of liver $\mathrm{Pu}$ were also achieved with $\mathrm{H}(2,2)$ CAM(C). Only H(2,2)-CAM(C) and 3,4,3-LIMeTAM reduced body $\mathrm{Pu}$ as much as an equimolar amount of oral 3,4,3-LICAM(C). The most favorable structure characteristics for in vivo $\mathrm{Pu}(\mathrm{IV})$ chelation, among the ligands reported here, were the simplest open PENTEN or linear backbone $[\mathrm{H}(2,2)$ - or $3,4,3-\mathrm{LI}]$ and the smallest simplest TAM metal-binding group, (MeTAM).

\section{Lanthanide Coordination}

\section{C.J. Sunderland and K.N. Raymond}

Previous work in this group has demonstrated some of the superior features of TREN-(N-Methyl-3-hydroxy-2pyridone) (TREN-(Me-3,2-HOPO)) as a complexing agent for the lanthanides. The thermodynamic stability is high, and the crystal structure of Gd(TREN-(Me-3,2-HOPO)) indicates that two water molecules are bound to the metal. Both these features are promising for MRI applications. This has prompted investigation of the structurally related (but more p-deficient) 5-hydroxy-4-pyrimidone (5,4HOPY) system. 'The slightly different ligand pKa's and synthetically easier heterocyclic core derivitization offered should allow for a subtle probe into the complexation properties of these heterocyclic hydroxy-amides.

Derivitization of the $1,4,7,10,13$-pentaazacyclopentadecane ([15]ane $\mathrm{N}_{5}$ ) macrocycle with (initially) three acetate and two acetamide pendant arms from the amine positions is being attempted. Two isomeric substitution patterns are possible: 1,4,7-triacetate-10,13-bisamide[15] ane $\mathrm{N}_{5}$ and 1,4,10-triacetate-7,13-bisamide-[15]aneN $\mathrm{N}_{5}$. The investigation will examine the extent to which the macrocyclic backbone can contribute to the stability of lanthanide polyaminocarboxylate/amide ligands. Comparison of the thermodynamic stability of both ligands isomers presents the possibility to examine the effect of structural variations of donor group positions within the same macrocyclic system. 


\section{Synthetic and Structural Studies of Actinides and Other Compounds}

Richard A. Andersen, Investigator

6. Crystal Structures of $\left[\left(\mathrm{Me}_{3} \mathrm{Si}\right)_{2} \mathrm{~N}_{4} \mathrm{U}_{2}[\mu-\right.$

$\mathrm{N}(\mathrm{H})($ mesityl $)]_{2}$ and $\left[\left(\mathrm{Me}_{3} \mathrm{Si}\right)_{2} \mathrm{~N}_{4} \mathrm{U}_{2}[\mu-\mathrm{N}(\text { p-tolyl })]_{2}\right.$;

Compounds with Asymmetrically Bridging Primary

Amide and Imide Groups

\section{J.L. Stewart and R.A. Andersen}

The trivalent uranium amide, $\left[\left(\mathrm{Me}_{3} \mathrm{Si}\right)_{2} \mathrm{~N}\right]_{4} \mathrm{U}_{2}[\mu$ $\mathrm{N}(\mathrm{H})$ (mesityl) $]_{2}$, was prepared by the acid-base reaction between $\left[\left(\mathrm{Me}_{3} \mathrm{Si}\right)_{2} \mathrm{~N}\right]_{3} \mathrm{U}$ and mesitylamine. The pKa's of $\left(\mathrm{Me}_{3} \mathrm{Si}\right)_{2} \mathrm{NH}$ and mesitylamine are not particularly helpful in predicting the outcome of the proton transfer reaction; this is probably due to the change in $\mathrm{pKa}$ upon coordination to the uranium center. $\mathrm{X}$-ray crystallographic analysis $\left(-75^{\circ} \mathrm{C}\right)$ of the compound, Figure $6-1$, shows that the mesitylamide (the amide hydrogen atom was located but not refined) asymmetrically bridges the two $\left[\left(\mathrm{Me}_{3} \mathrm{Si}\right)_{2} \mathrm{~N}_{2} \mathrm{U}\right.$ centers with U-N distances of $2.453(3) \AA$ and $2.646(3) \AA$ and a U-N-U angle of $102.9(1)^{\circ}$. The tetravalent uranium organoimide, $\left[\left(\mathrm{Me}_{3} \mathrm{Si}_{2}\right)_{2} \mathrm{~N}_{4} \mathrm{U}_{2}[\mu-\mathrm{N}(\text { p-tolyl })]_{2}\right.$, was prepared in the reaction of $\left[\left(\mathrm{Me}_{3} \mathrm{Si}\right)_{2} \mathrm{~N}\right]_{3} \mathrm{UCl}$ and $\mathrm{LiN}(\mathrm{H})$ (p-tolyl), a reaction which is thought to involve a proton transfer step. $\mathrm{X}$-ray crystallographic analysis $\left(-118^{\circ} \mathrm{C}\right)$ of the compound, Figure 6-2, shows that the p-tolylimide asymmetrically bridges the two $\left[\left(\mathrm{Me}_{3} \mathrm{Si}\right)_{2} \mathrm{~N}\right]_{2} \mathrm{U}$ centers with $\mathrm{U}-\mathrm{N}$ distances

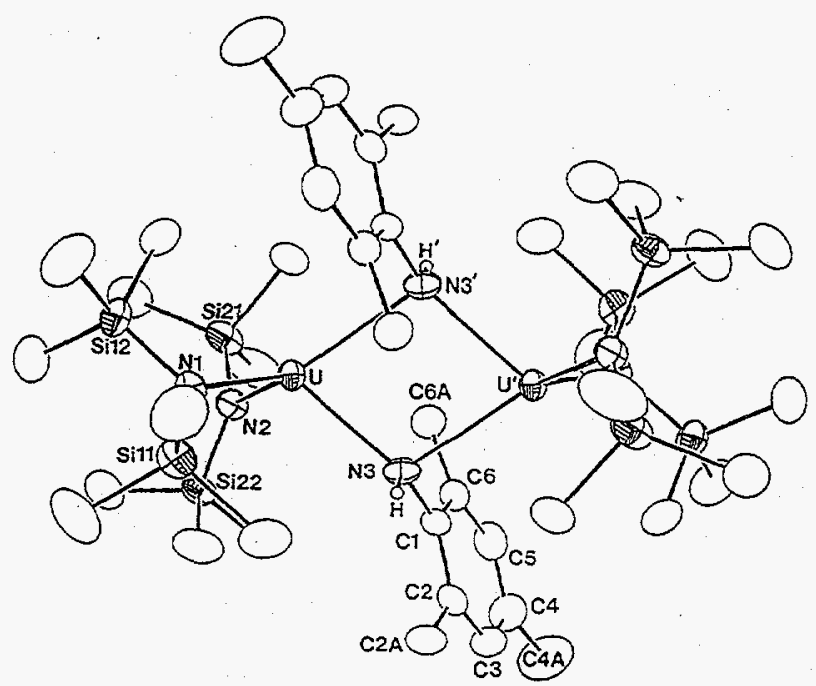

Figure 6-1. ORTEP diagram of $\mathrm{U}_{2}\left[\mathrm{~N}\left(\mathrm{SiMe}_{3}\right)_{2}\right]_{4}[\mu-\mathrm{N}(\mathrm{H})(2,4,6-$

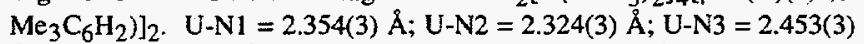
$\AA$; $\mathrm{U}-\mathrm{N} 3=2.646(3) \AA$. . (XBD 951-125)

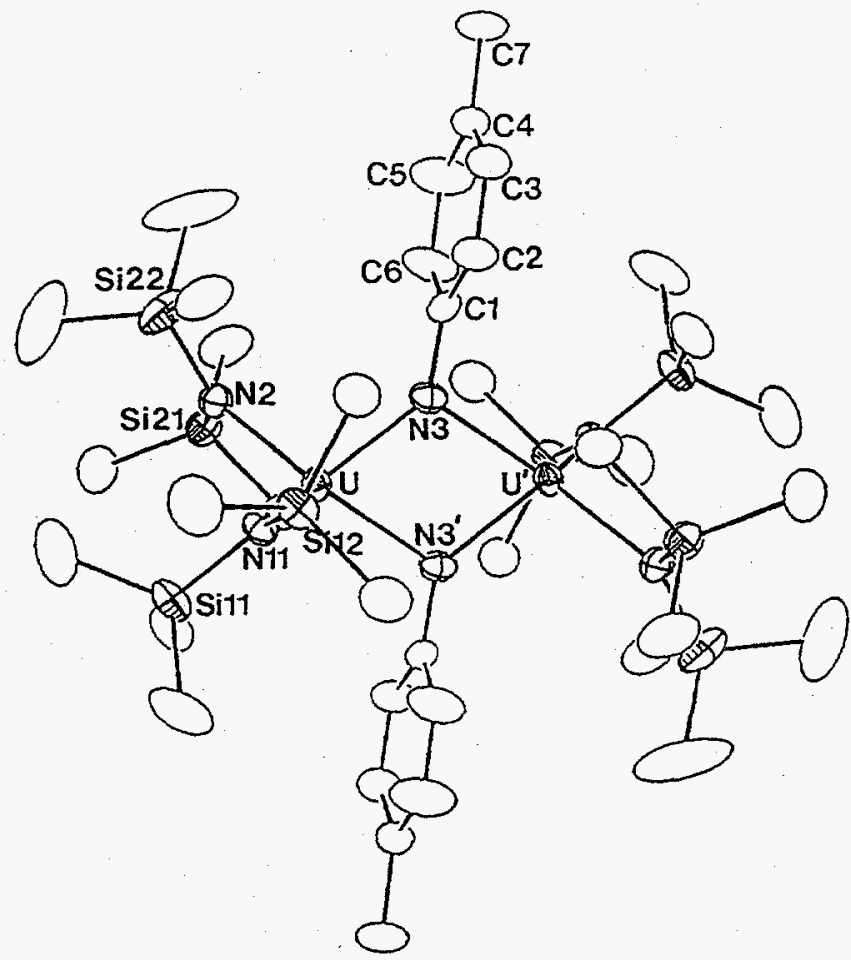

Figure 6-2. ORTEP diagram of $\mathrm{U}_{2}\left[\mathrm{~N}\left(\mathrm{SiMe}_{3}\right)_{2}\right]_{4}[\mu-\mathrm{N}(\mathrm{p}-\mathrm{Tolyl})]_{2}$. U-N1 $=2.293(3) \AA \AA \mathrm{U}-\mathrm{N} 2=2.274(2) \AA ; \mathrm{U}-\mathrm{N} 3=2.17(3) \AA ; \mathrm{U}-\mathrm{N} 3=2.278(3) \AA$. (XBD 951-124)

of 2.378(3) $\AA$ and 2.172(2) $\AA$ and a U-N-U angle of $105.8(1)^{\circ}$. The asymmetry is ascribed to electronic (hybridization) effects. Magnetic susceptibility studies from $5-280 \mathrm{~K}$ on both compounds show no magnetic interaction between the uranium centers.

7. Preparation of Monomeric $\left(\mathrm{Me}_{5} \mathrm{C}_{5}\right)_{2} \mathrm{VO}$ and $\left(\mathrm{Me}_{5} \mathrm{C}_{5}\right)_{2} \mathrm{Ti}(\mathrm{O})(\mathrm{L})$ and Their Decomposition to $\left(\mathrm{Me}_{5} \mathrm{C}_{5}\right)_{4} \mathrm{M}_{4}(\mu-\mathrm{O})_{6}$

\section{M.R. Smith III, P.T. Matsunaga, and R.A. Andersen}

The vanadium metallocene, $\left(\mathrm{Me}_{5} \mathrm{C}_{5}\right)_{2} \mathrm{~V}$, reacts with $\mathrm{N}_{2} \mathrm{O}$ (4 atm, room temperature) to give $\left(\mathrm{Me}_{5} \mathrm{C}_{5}\right)_{2} \mathrm{VO}\left(\mathrm{v}_{\mathrm{Vo}}\right.$ $\left.=855 \mathrm{~cm}^{-1}\right)$ as green-brown crystals from hexane. The vanadyl is monomeric in the gas phase $\left(\mathrm{M}^{+}\right.$in the mass spectrum) and paramagnetic with $\mu_{\mathrm{eff}}=1.95 \mathrm{BM}(\theta=$ $0.49 \mathrm{~K}$ from $5-300 \mathrm{~K}$ ). An isotropic EPR spectrum is observed in methylcyclohexane at room temperature which consists of eight-lines with $\mathrm{g}_{\text {iso }}=1.9844$ and $\mathrm{A}_{\text {iso }}=25 \mathrm{G}$. Similarly, reaction of $\left(\mathrm{Me}_{5} \mathrm{C}_{5}\right)_{2} \mathrm{Ti}\left(\mathrm{C}_{2} \mathrm{H}_{4}\right)$ with $\mathrm{N}_{2} \mathrm{O}$ in a mixture of pyridine-tetrahydrofuran yields monomeric, diamagnetic $\left(\mathrm{Me}_{5} \mathrm{C}_{5}\right)_{2} \mathrm{Ti}(\mathrm{O})(\mathrm{py})$ as orange crystals $\left(v_{\mathrm{TiO}}=\right.$ $\left.852 \mathrm{~cm}^{-1}, v_{\mathrm{Ti}^{18} \mathrm{O}}=818 \mathrm{~cm}^{-1}\right)$. The derivative, $\left(\mathrm{Me}_{5} \mathrm{C}_{5}\right)_{2} \mathrm{Ti}(\mathrm{O})(4-$ phenylpyridine), crystallizes as a toluene 


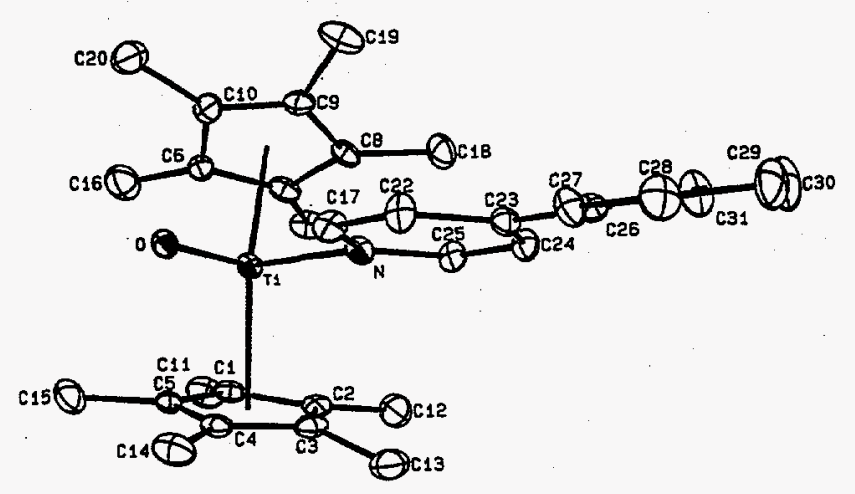

Figure 7-1. ORTEP diagram of $\left(\mathrm{Me}_{5} \mathrm{C}_{5}\right)_{2} \mathrm{Ti}(\mathrm{O})$ (4-phenylpyridine) (toluene), toluene solvate not shown. Ti-C (av) $=2.51 \pm 0.05 \AA$ (rms deviation); $\mathrm{Ti}-\mathrm{Cp}$ (centroid) $=2.20 \AA$ 政 $\mathrm{T}-\mathrm{O}=1.665(3) \AA ; \mathrm{Ti}-\mathrm{N}=$ 2.215(4); Cp (centroid) $-\mathrm{Ti}-\mathrm{Cp}$ (centroid) $=108^{\circ} ; \mathrm{Cp}$ (centroid) $-\mathrm{TiN}=$ $103^{\circ} ; \mathrm{O}-\mathrm{Ti}-\mathrm{N}=90.8(1)^{\circ}$. (XBD 951-122)

solvate, Figure 7-1. The geometry about the titanium is pseudotetrahedral with $\mathrm{Ti}-\mathrm{O}=1.665(3) \AA$. Both oxometallocenes decompose ( $M=V$ at room temperature, $M=$ $\mathrm{Ti}$ with gentle warming) to the known tetranuclear compounds $\left(\mathrm{Me}_{5} \mathrm{C}_{5}\right)_{4} \mathrm{M}_{4}(\mu-\mathrm{O})_{6}, \mathrm{M}=\mathrm{T}, \mathrm{V}$. Mass spectroscopic studies of the ${ }^{18} \mathrm{O}$-labeled compounds show that all of the label in the tetranuclear compounds are derived from the monomeric precursors in a complex net reaction that results in the $\mathrm{MO}$ unit going to the $\mathrm{M}_{4} \mathrm{O}_{6}$ unit.

\section{Dinuclear Titanium (III) Complexes; Exchange Coupling in $\left(\mathrm{Cp}_{2} \mathrm{Ti}\right)_{2}(\mu-\mathrm{O})$ (Publication 19)}

\section{W.W. Lukens, Jr. and R.A. Andersen}

Addition of one-half of an equivalent of water in tetrahydrofuran to $\mathrm{Cp}_{3} \mathrm{Ti}$ in tetrahydrofuran yields the known bridging oxo derivative of $\mathrm{Ti}(\mathrm{III}),\left(\mathrm{Cp}_{2} \mathrm{Ti}\right)_{2}(\mu-\mathrm{O})$, in high yield. Use of labeled water gives $\left(\mathrm{C}_{2} \mathrm{Ti}_{2}\left(\mu-{ }^{18} \mathrm{O}\right)\right.$. The EPR spectrum of a toluene glass at $2 \mathrm{~K}$ gives a spectrum due to a triplet ground state with $\mathrm{g}_{\|}=1.979, \mathrm{~g}_{\perp}=$ 1.981 , and $\mid \mathrm{DI}=0.0249 \mathrm{~cm}^{-1}$. The variable temperature magnetic susceptibility above $20 \mathrm{~K}$ shows intramolecular ferromagnetic coupling and weak intermolecular ferromagnetic coupling; the intramolecular coupling constant is $8.3 \mathrm{~cm}^{-1}$. Below $20 \mathrm{~K}$, the susceptibility is field dependent. An orbital diagram is presented that accounts for the magnetic behavior in the $\mathrm{d}^{1} \mathrm{~d}^{1}$ dinuclear metallocene, see Figure 8-1.

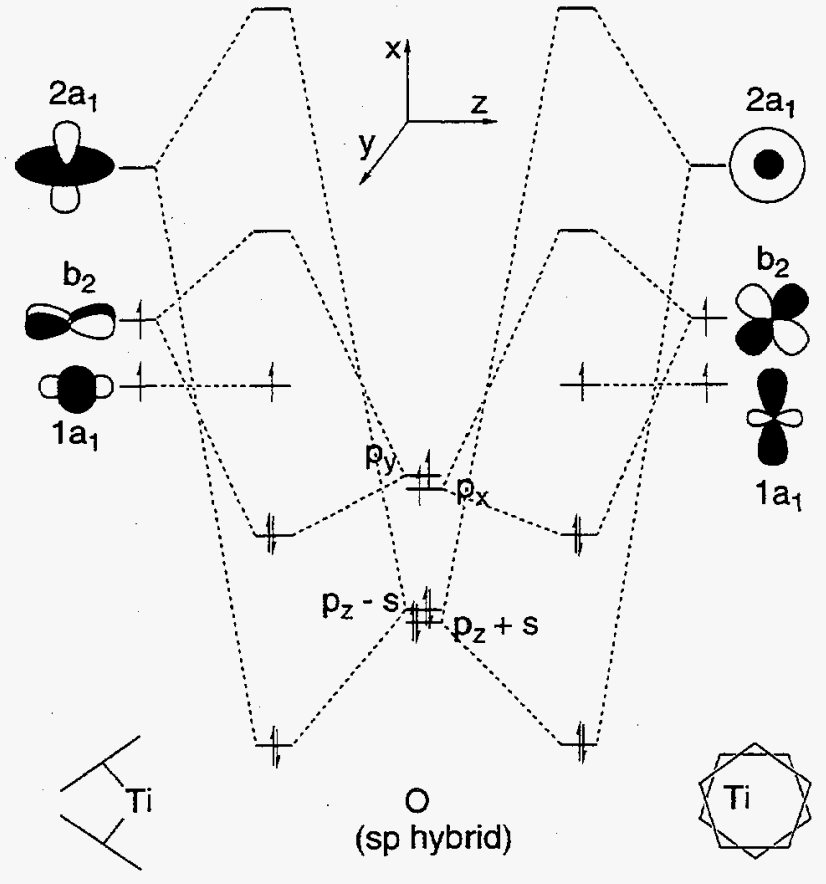

Figure 8-1. A qualitative molecular orbital diagram for $\left(\mathrm{Cp}_{2} \mathrm{Ti}\right)_{2}(\mu-\mathrm{O})$. (XBD 9505-02116)

\section{Bis(pentamethylcyclopentadienyl)titanium Amide}

\section{E. Brady, W. Lukens, J.R. Telford, and G. Mitchell}

The title compound was synthesized as part of an investigation of metal-ligand $\pi$ bonding in titanium(III) compounds. Since the electronically favored orientation of the $\mathrm{NH}_{2}$ group is the most sterically hindered, the relative strength of the electronic and steric effects can be examined. If steric effects dominate, the plane of the $\mathrm{NH}_{2}$ ligand should be perpendicular to the plane formed by the titanium atom and the two centroids of the pentamethylcclopentadienyl ligands. On the other hand, if electronic effects dominate, the titanium atom, and the two centroids, and the $\mathrm{NH}_{2}$ group, acting as a p-donor, will all lie in the same plane. The $\mathrm{NH}_{2}$ group is oriented to maximize the $\pi$-interaction between nitrogen and titanium. The Ti-N bond length is short [1.944(2) $\AA$ ], consistent with increased $\pi$-bonding, see Figure 9-1. 


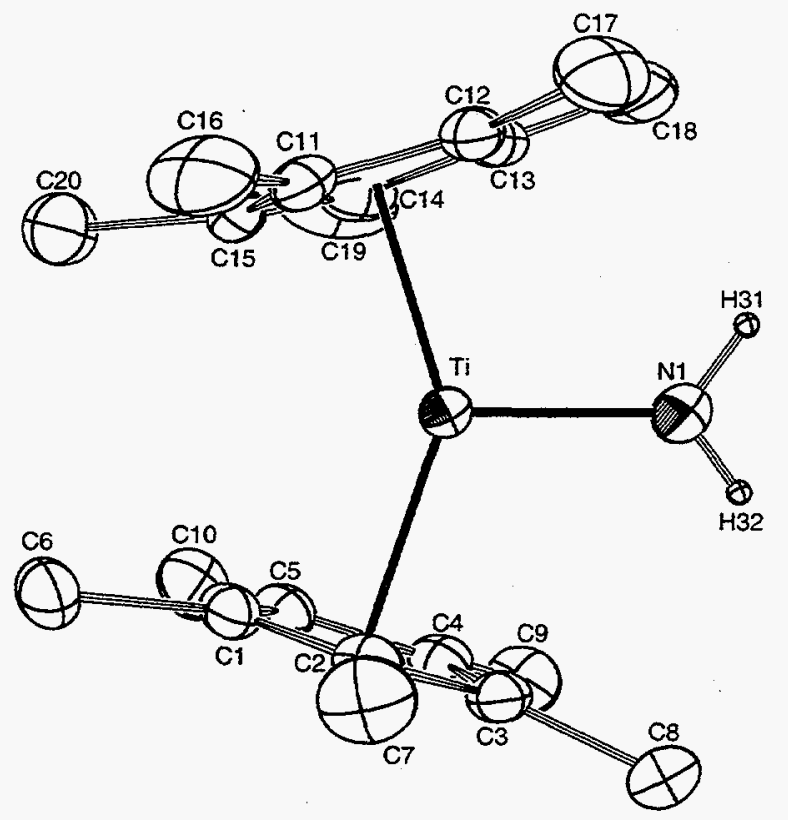

Figure 9-1. ORTEP diagram of $\left(\mathrm{Cp}^{*}{ }_{2} \mathrm{TiNH}_{2}\right.$ with $50 \%$ thermal ellipsoids. Ti-N $=1.944(2) \AA ; \mathrm{Cp}$ (centroid) $=2.07 \AA$. (XBD 951-121)

10. Tris(trimethylsilylcyclopentadienyl)zirconium Chloride

\section{W. Lukens}

The title compound is a bent metallocene with two $\eta^{5}$ cyclopentadienyl ligands and one $\eta^{1}$-cyclopentadienyl ligand. The $\mathrm{Zr}-\mathrm{C}$ bond length to the $\eta^{1}$-cyclopentadienyl ligand is $2.396(5) \AA$, and the $\mathrm{Zr}-\mathrm{Cl}$ bond length is 2.442(1) $\AA$, see Figure 10-1.

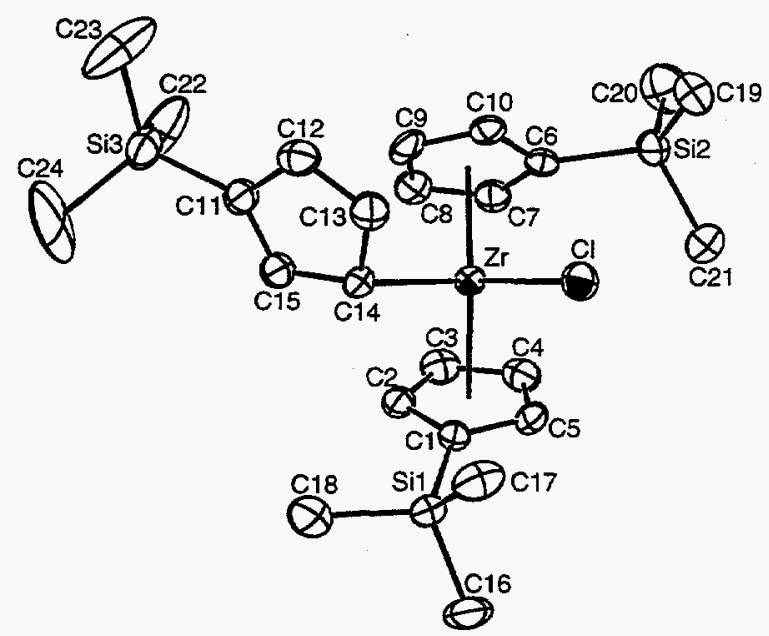

Figure 10-1. ORTEP diagram of $\left(\mathrm{Me}_{5} \mathrm{SiC}_{5} \mathrm{H}_{4}\right)_{3} \mathrm{ZrCl}$ with $50 \%$ thermal ellipsoids. Only one set of the disordered atoms, C22, C23, and C24, is shown. $\mathrm{Zr}-\mathrm{Cl}=2.442(1) \AA ; \mathrm{Zr}-\mathrm{C} 14=2.396(5) \AA ; \mathrm{Zr}-\eta^{5}-\mathrm{C}($ ave $)=2.52(3)$ Å. (XBD 951-123)

\section{Physical and Spectroscopic Properties}

Norman M. Edelstein, Investigator
11. Spectroscopic Study and Crystal Field Analysis of $\mathrm{Cm}^{3+}$ in the Cubic Symmetry Site of $\mathrm{ThO}_{2}$ (Publication 6)

\section{P. Thouvenot, S. Hubert, and N. Edelstein}

$\mathrm{Cm}$, element number 96 in the Periodic Table, occurs in the center of actinide series and its trivalent ion; $\mathrm{Cm}^{3+}$ (radon core, $5 f^{7}$ ) has a half-filled shell. The differences in the electronic structure between its lanthanide counterpart $\mathrm{Gd}^{3+}, 4 \mathrm{f}^{7}$, and $\mathrm{Cm}^{3+}$ demonstrate the effect of the much greater spin-orbit coupling that occurs in the $5 \mathrm{f}$ series. The $\mathrm{Gd}^{3+}$ ground electronic term is an almost pure ${ }^{8} \mathrm{~S}_{7 / 2}$ configuration. Although this $\mathrm{J}=7 / 2$ ion splits in a crystalline field into at most four doublets, the total extent of the splitting is on the order of $\sim 0.5 \mathrm{~cm}^{-1}$. The $\mathrm{Cm}^{3+}$ ion has a much larger spin-orbit coupling constant, and its ground term is only $\sim 80 \%{ }^{8} \mathrm{~S}_{7 / 2}$. As a consequence of the mixing of higher-lying states into the ground term by the spin-orbit coupling interaction (intermediate coupling), the ground state splitting of the lowest $J=7 / 2$ state of $\mathrm{Cm}^{3+}$ in solids is considerably larger than in $\mathrm{Gd}^{3+}, \sim 2-50 \mathrm{~cm}^{-1}$.

Fluorescence and excitation spectra of $\mathrm{Cm}^{3+}$ diluted in $\mathrm{ThO}_{2}$ powder are reported at room and low temperatures. The observed $\mathrm{Cm}^{3+}$ spectra are from the cubic symmetry lattice site and are assigned to phonon-assisted electric dipole transitions and to magnetic dipole transitions. From these assignments the crystal field parameters $\mathrm{B}_{0}^{4}=-6436$ $\mathrm{cm}^{-1}$ and $B_{0}^{6}=1195 \mathrm{~cm}^{-1}$ have been determined. The experimental energy levels for the ground term determined from the optical data (see Figure 11-1) and earlier electron paramagnetic resonance spectra are in good agreement with the calculated values.

12. Comparison of the Electronic Structure of the Lanthanides and Actinides (Publication 24)

\section{N.M. Edelstein}

The lanthanide series consists of the fourteen elements following lanthanum in the periodic table and is formed by the successive addition of a $4 \mathrm{f}$ electron to the electronic configuration of lanthanum. Because the $4 \mathrm{f}$ shell is an inner shell, the chemistry of the lanthanide ions are in general very similar. Although the chemical properties of the early actinide ions are quite different from that of the later actinide ions and the lanthanide series, the actinide 

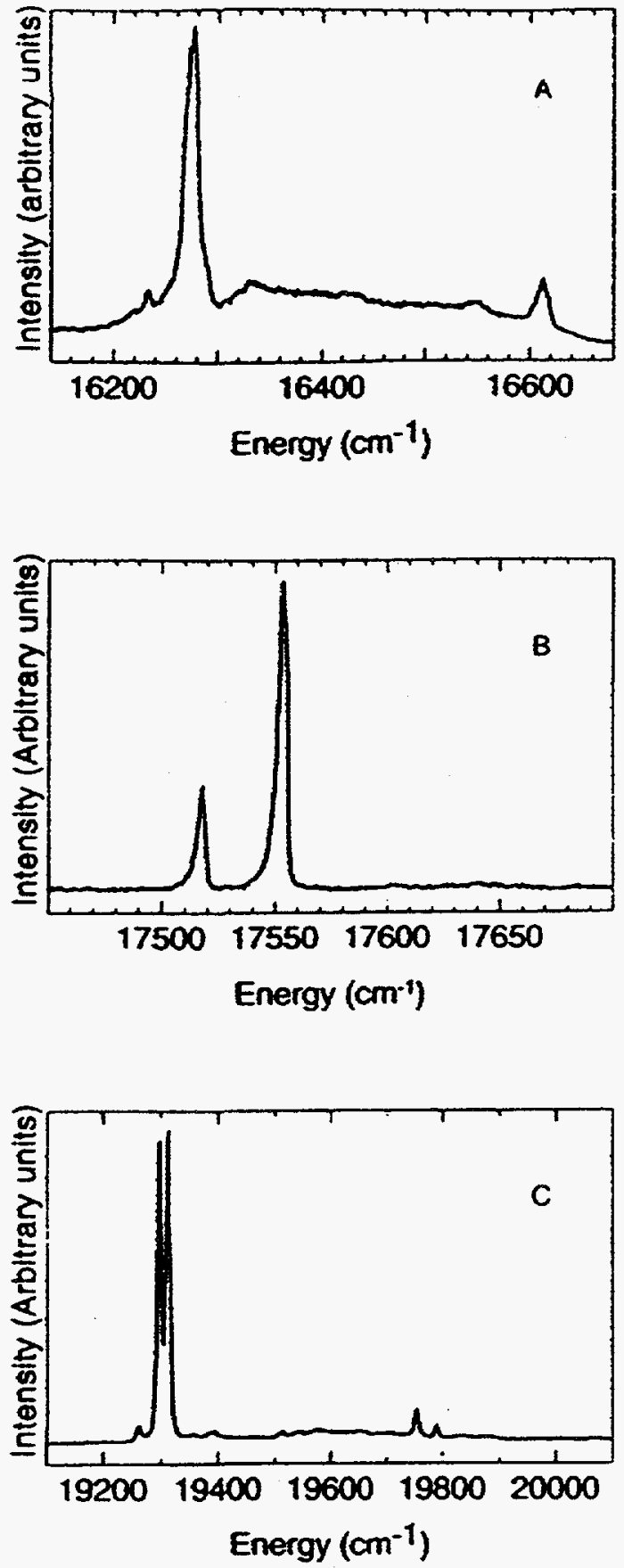

Figure 11-1. Excitation spectra of $\mathrm{Cm}^{3+} / \mathrm{ThO}_{2}$ at $10 \mathrm{~K}$. (A) $16100-$ $16700 \mathrm{~cm}^{-1}$ region; (B) $17400-17700 \mathrm{~cm}^{-1}$ region showing the crystal field splitting between the first (lowest) and the third crystal field level of the ground term; (C) $19100-20000 \mathrm{~cm}^{-1}$ region showing the splitting between the first and second crystal field levels of the ground term $\left(\sim 19300 \mathrm{~cm}^{-1}\right)$ as well as the splitting between the first and third levels $\left(-19790 \mathrm{~cm}^{-1}\right)$. (XBD 951-126)

series, in analogy with the lanthanide series, is defined as the fourteen elements following actinium in the periodic table. The $4 \mathrm{f}$ orbitals in the lanthanide series are inner orbitals and do not participate in chemical bonding. In the

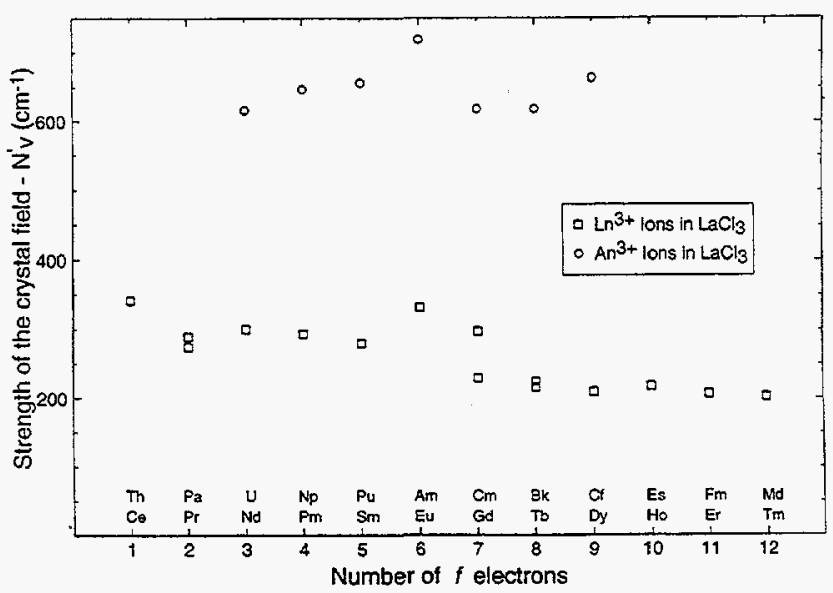

Figure 12-1. A comparison of the $\mathrm{N}_{\mathrm{v}}{ }^{\prime}$ values obtained from the crystalfield parameters for $\mathrm{Ln}^{3+}$ and $\mathrm{An}^{3+}$ in $\mathrm{LaCl}_{3}$ (XBD 9505-02117)

early actinides the $5 f$ orbitals are more extended and very close in energy to the $6 \mathrm{~d}$ orbitals. As the atomic number increases, the $5 f$ orbitals become more localized and progressively lower in energy relative to the $6 \mathrm{~d}$ configuration.

This paper reviews the electronic structure of the $4 \mathrm{f}$ and $5 f$ compounds. To this end the optical and magnetic analyses of similar compounds are compared. In most cases this entails comparing trivalent actinides with trivalent lanthanide compounds although the $\mathrm{f}^{1}$ configuration ( $\mathrm{Ce}^{3+}$ diluted in $\mathrm{Cs}_{2} \mathrm{NaYCl}_{6}$ and $\mathrm{Pa}^{4+}$ diluted in $\mathrm{Cs}_{2} \mathrm{ZrCl}_{6}$ ) will be treated in detail. In general the ground state for lanthanide and actinide compounds with an equal number of $f$ electrons $\left(f^{n}\right)$ and the same coordination about the metal ion is similar, although the total crystal field splitting in the actinides is approximately twice as great as for the lanthanides (see Figure 12-1). The half-filled shell, $\mathrm{f}^{7}$, is a special case with a relatively large ground state splitting in the $5 \mathrm{f}^{7}$ ground term caused by the effects of the much larger spin-orbit coupling.

\section{The Synthesis and Electronic Structure of $\mathrm{Pa}\left(\mathrm{BH}_{3} \mathrm{CH}_{3}\right)_{4}$ (Publication 25)}

\section{W.K. Kot and N.M. Edelstein}

The nonaqueous chemistry of $\mathrm{Pa}$ resembles somewhat that of $\mathrm{Nb}$ and $\mathrm{Ta}$. The most stable oxidation state of $\mathrm{Pa}$ is pentavalent, its tetravalent state is unstable towards oxidation by air, and the trivalent state has been reported but not well-established. The classical inorganic chemistry of $\mathrm{Pa}$ is well-known, but only a few molecular compounds (three organometallic compounds and $\mathrm{Pa}\left(\mathrm{BH}_{4}\right)_{4}$ ) had been reported. The obvious synthetic route to organometallic 
and borohydride compounds is by the reaction between $\mathrm{PaCl}_{4}$ and the appropriate ligand reagents. A modification of an earlier synthesis for $\mathrm{PaCl}_{5}$ from hydrated $\mathrm{Pa}_{2} \mathrm{O}_{5}$ has been developed. It was found that $\mathrm{PaCl}_{5}$ is soluble in chlorobenzene, toluene, or benzene with a solubility of up to $25 \mathrm{mg}$ of $\mathrm{PaCl}_{5}$ in $5 \mathrm{ml}$ of toluene. Reaction of $\mathrm{PaCl}_{5}$ dissolved in toluene with excess $\mathrm{LiBH}_{4}$ gave $\mathrm{Pa}\left(\mathrm{BH}_{4}\right)_{4}$ in minutes from which a yield of $45 \%$ was obtained after sublimation. Thus it is possible to go directly from the pentavalent state directly to tetravalent compounds. Similar reactions of $\mathrm{PaCl}_{5}$ with excess $\mathrm{MeCpNa}$ or $\mathrm{LiBH}_{3} \mathrm{CH}_{3}$ gave the compounds $(\mathrm{MeCp})_{4} \mathrm{~Pa}$ or $\mathrm{Pa}\left(\mathrm{BH}_{3} \mathrm{CH}_{3}\right)_{4}$. These new compounds were identified by their NMR spectra.

The series of compounds $\mathrm{M}\left(\mathrm{BH}_{3} \mathrm{CH}_{3}\right)_{4}(\mathrm{M}=\mathrm{Zr}$, Th, $\mathrm{U}$, and $\mathrm{Np}$ ) had been synthesized and structurally characterized earlier. These four molecules are monomeric in the solid state and for each molecule the metal atom is tetrahedrally coordinated to the methylborohydride group through tridentate hydrogen bridge bonds (see Figure 13-1). Solution NMR studies of the paramagnetic members of the $\mathrm{M}\left(\mathrm{BH}_{3} \mathrm{CH}_{3}\right)_{4}$ molecules are of special interest because the $T_{d}$ molecular symmetry of the complexes means that the dipolar contribution to the paramagnetic shifts vanishes. Thus the interactions between the unpaired magnetic electrons and the ligand nuclei (the so-called Fermi contact shift) can be directly measured.

The measured temperature dependencies of the ${ }^{1} \mathrm{H}$ and ${ }^{11} \mathrm{~B}$ shifts of the $\mathrm{T}_{\mathrm{d}} \mathrm{Pa}\left(\mathrm{BH}_{3} \mathrm{CH}_{3}\right)_{4}$ have been analyzed. For

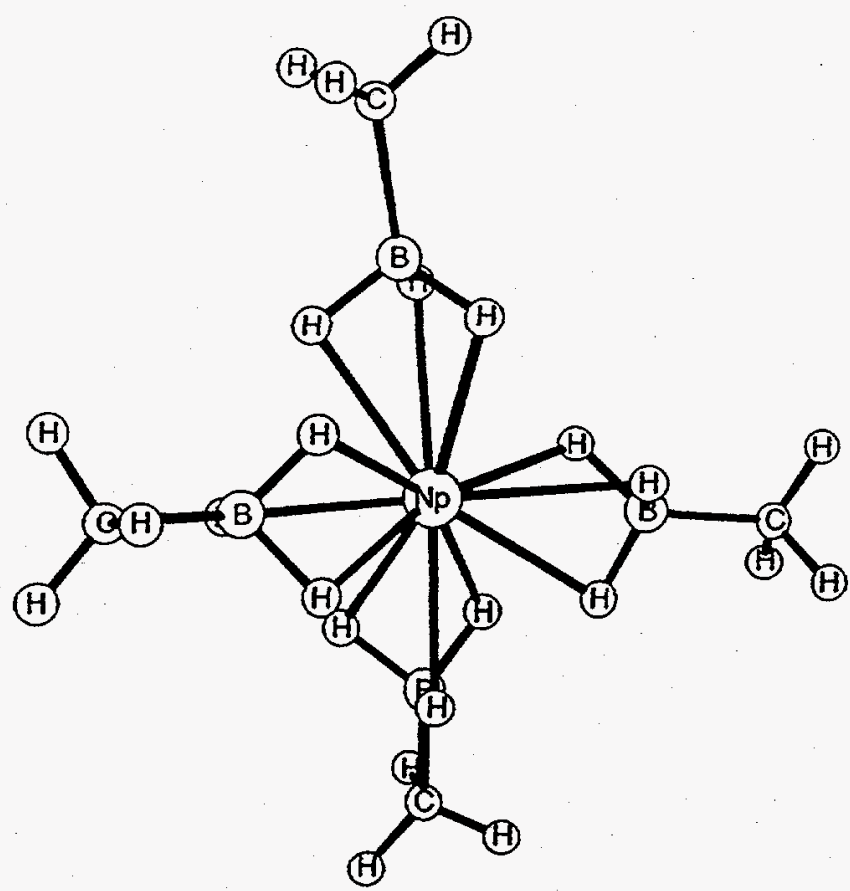

Figure 13-1. Schematic view of $\mathrm{Np}\left(\mathrm{BH}_{3} \mathrm{CH}_{3}\right)_{4}$ showing the $\mathrm{T}_{\mathrm{d}}$ symmetry about the metal ion. (XBD 951-128)

the Pa compound, the ${ }^{1} \mathrm{H}$ NMR peaks of both the bridging and terminal protons shift to lower field as the temperature is decreased in contrast with the data for $\mathrm{U}\left(\mathrm{BH}_{3} \mathrm{CH}_{3}\right)_{4}$ and $\mathrm{Np}\left(\mathrm{BH}_{3} \mathrm{CH}_{3}\right)_{4}$ (Figure 13-2). These observations for
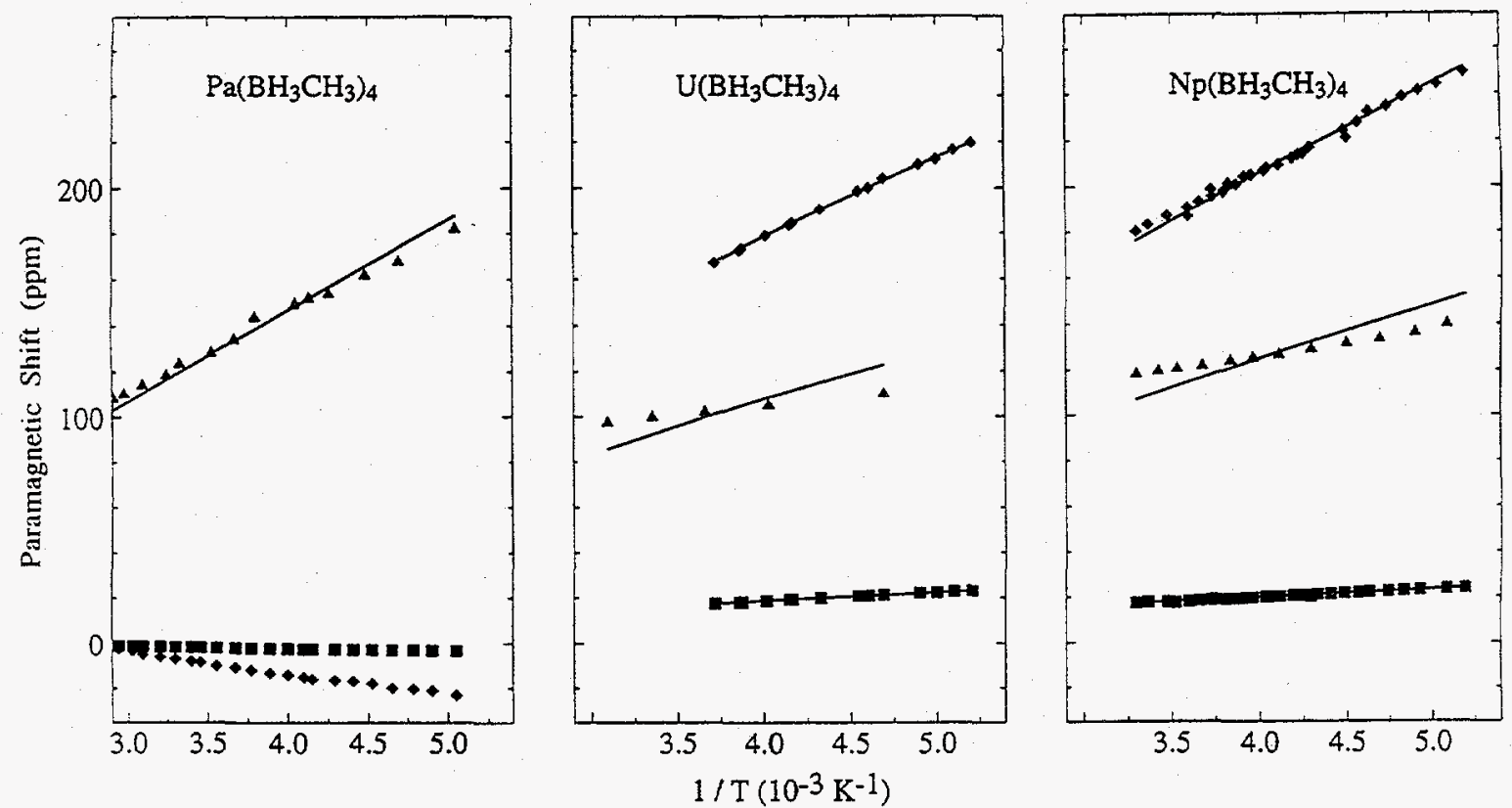

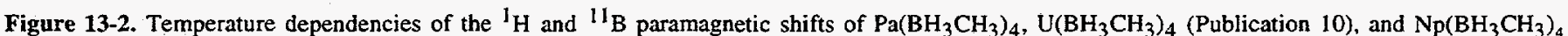
(Publications 10 and 11 ). The symbols are as follows: $\bullet$ bridging proton, $\mathbf{a}$ : terminal proton, $A:{ }^{11} \mathrm{~B}$. The lines represent least-squares fits. (XBD $951-127$ ) 
$\mathrm{Pa}\left(\mathrm{BH}_{3} \mathrm{CH}_{3}\right)_{4}$ are inconsistent with the spin-polarization mechanism that was used previously to analyze the temperature-dependent proton shifts in $\mathrm{U}\left(\mathrm{BH}_{3} \mathrm{CH}_{3}\right)_{4}$ and $\mathrm{Np}\left(\mathrm{BH}_{3} \mathrm{CH}_{3}\right)_{4}$. The $\mathrm{Pa}^{4+}$ ion has one $5 \mathrm{f}$ electron in its ground configuration outside closed shells that has a relatively extended radial distribution and may undergo extensive interaction with the ligands. A second mechanism that could be important in transferring spin density to both ${ }^{1} \mathrm{H}$ and ${ }^{11} \mathrm{~B}$ is covalency but a much more detailed analysis is needed.

14. Synthesis, Crystal Structure, Magnetism, and Absorption Spectra of $A_{2} U X_{5}$ Type Halides $(A=K$, $\mathrm{Rb} ; \mathrm{X}=\mathrm{Cl}, \mathrm{Br}, \mathrm{I}$ ) (Publication 7)

K. Krämer, H.U. Güdel, G. Meyer, T. Heuer, N.M. Edelstein, B. Jun, L. Keller, P. Fischer, E. Zych and J. Drozdzynski

The ternary uranium(III) halides $\mathrm{A}_{2} \mathrm{UX}_{5}(\mathrm{~A}=\mathrm{K}, \mathrm{Rb}$; $\mathrm{X}=\mathrm{Cl}, \mathrm{Br}, \mathrm{I})$ have been prepared from the binary components $\mathrm{AX}$ and $\mathrm{UX}_{3}$ in sealed tantalum containers. These compounds all crystallize with the $\mathrm{K}_{2} \mathrm{PrCl}_{5} / \mathrm{Y}_{2} \mathrm{HfS}_{5}$ type of structure. Lattice constants for ambient temperature are reported. Single-crystal structure refinements were undertaken for $\mathrm{K}_{2} \mathrm{UI}_{5}$ and $\mathrm{Rb}_{2} \mathrm{UCl}_{5}$. Magnetic susceptibility data were recorded with a SQUID magnetometer from liquid helium to room temperature. One-dimensional (intrachain) and three-dimensional antiferromagnetic ordering occur at low temperatures dependent upon the $\mathrm{U}^{3+}-\mathrm{U}^{3+}$ distance. Absorption spectra were recorded between 4000 and $28000 \mathrm{~cm}^{-1}$. They show $\mathrm{f} \rightarrow \mathrm{f}$ transitions typical for $\mathrm{U}^{3+}$ and, depending on the halide, very strong $\mathrm{f} \rightarrow \mathrm{d}$ transitions above 14000 to 15000 $\mathrm{cm}^{-1}$, respectively.

\section{Actinide Synchrotron Radiation Program}

\author{
David K. Shuh and Norman M. Edelstein, Investigators
}

\section{INTRODUCTION}

Synchrotron Radiation (SR) techniques in both the soft $\mathrm{x}$-ray and $\mathrm{x}$-ray regimes, along with the advent of third generation light sources, have been recognized as valuable new resources for the experimental investigation of a wide range of scientific and technological issues concerning actinide materials. The soft $\mathrm{x}$-ray SR thrust utilizes the unique high-brightness attributes of the Advanced Light
Source (ALS) in conjunction with a scanning photoemission microscope to perform the first SR-based investigations of solid state transuranic materials in the 50$1500 \mathrm{eV}$ energy regime. The development and refinement of actinide materials preparation methodologies to make full use of the ALS capabilities are under way.

$S R$ investigations in the $x$-ray regime continue to be pursued and exploited since radioactive and hazardous materials may be safely contained for in situ investigations at these energies. X-ray Absorption Spectroscopy (XAS) techniques are useful, nondestructive probes employed to ascertain oxidation states of materials by X-ray Absorption Near Edge Structure (XANES) and structural information from Extended X-ray Absorption Fine Structure (EXAFS). XAS methodologies are compatible with low concentration samples and are also highly amenable for studies of solutions, both of which are of the utmost importance for environmental purposes. Thus, these methodologies are well-suited for detailed investigations of actinide materials, fission products, and other environmentally relevant systems.

The development of $\mathrm{x}$-ray synchrotron radiation detectors, instrumentation, and sample handling techniques is essential for the succesful research implementation of XAS methodologies to relevant actinide systems. A monolithic four-element germanium solid state detector has been specifically fabricated for use in SR environments with low concentration actinide solution samples, and the initial performance trials of the detector have been successful. Continuing efforts in these fundamental areas permit better utilization of beamtime, exploration of actinide materials systems never before possible, and enables the study of systems with inherently low signal to background.

\section{Environmental Applications of XANES:}

Speciation of Tc in Cement After Chemical Treatment and Se After Bacterial Uptake ${ }^{\dagger}$ (Publication 30)

\section{D.K. Shuh, N. Kaltsoyannis, J.J. Bucher, N.M. Edelstein, S.B. Clark, H. Nitsche, T. Reich, E.A. Hudson, I. Almahamid, P. Torretto, W. Lukens, K. Roberts, B.C. Yee, D.E. Carlson, A. Yee, B.B. Buchanan, T. Leighton, W.-S. Yang, and J.C. Bryan}

XANES has been employed to evaluate the efficacy of a process designed to encapsulate and reduce $\mathrm{TcO}_{4}{ }^{-}$in cement matrices, thereby immobilizing Tc. The oxidation state of Se following bioremediation of Se by bacteria has also been determined by XANES. The XANES measurements were performed at the Stanford Synchrotron Radiation Laboratory (SSRL) and the National Synchrotron Light Source (NSLS) at the respective $\mathrm{K}$ edges of Tc 
$(21.0 \mathrm{keV})$ and $\mathrm{Se}(12.7 \mathrm{keV})$. Comparison of the XANES spectra (shown in Figure 15-1) of Tc in untreated cement to Tc in slag treated cement and to the chemical shifts of reference materials, shows that the oxidation state of $\mathrm{Tc}$ is the same in both cements. Thus, the addition of a reducing agent to the cement formulation does not significantly reduce the $\mathrm{TcO}_{4}^{-}$.

The common soil bacterium, Bacillus subtilis, is known to incorporate $\mathrm{Se}$ on or within the cell wall when exposed to a Se(IV) solution. The Se XANES spectra of $B$. subtilis, as well as bacillus isolated from selenium rich soil, show that the organisms reduce selenite to the red allotrope of elemental Se.

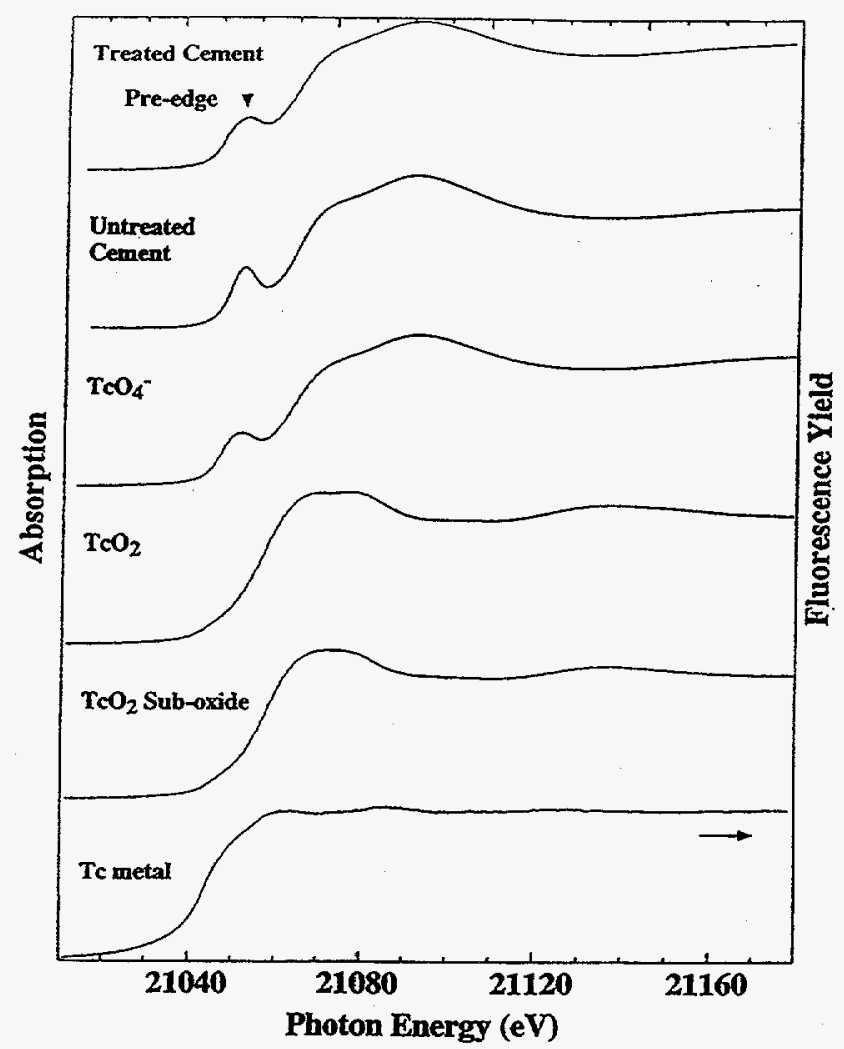

Figure 15-1. Technetium $K$ edge XANES spectra from the treated and untreated $\mathrm{Tc}$-containing cements, as well the Tc reference materials. The pre-edge feature, indicative of the tetrahedral coordination of the $\mathrm{TcO}_{4}^{-}$ species, is clearly evident. The spectra have been normalized and aligned with respect to the Tc metal edge at 21,044 eV. (XBD 951-129)

\footnotetext{
${ }^{\dagger}$ This work was performed in part at SSRL and NSLS, which are operated by the Department of Energy. Divisions of Chemical and Materials Sciences.
}

16. First Results From the SpectroMicroscopy Beamline at the Advanced Light Source ${ }^{\dagger}$ (Publication 31)

\section{J.D. Denlinger, E. Rotenberg, T. Warwick, G. Visser, J. Nordgren, J.-H. Guo, P. Skytt, S.D. Kevan, K.S. McCutcheon, J.G. Tobin, D.K. Shuh, J.J. Bucher, N.M. Edelstein, and B.P. Tonner}

The SpectroMicroscopy Facility at the Advanced Light Source is based on a high-brightness, high-resolution beamline, and the first ultra ESCA experiment to be performed was a proof-of-principle demonstration of the analysis of a minute quantity of a radioactive transuranic sample. In this experiment approximately $1.25 \mu$ grams of the isotope ${ }^{248} \mathrm{Cm}$ was deposited in the form of a curium oxide distributed over a spot $2.5 \mathrm{~mm}$ in diameter on a platinum disk. The total activity of the curium sample was less than $20 \mathrm{nCi}$, most of which arises from a trace ${ }^{246} \mathrm{Cm}$ impurity in the ${ }^{248} \mathrm{Cm}$. Spectra were collected for photoelectrons emitted from the $\mathrm{Cm} 4 f, 4 d$, and valence electron energy levels as well as the $O 1$ s level. A representative $\mathrm{Cm} 4 f$ spectrum is shown in Figure 16-1. Previously, $\mathrm{Cm}$ spectra have been recorded with fixedenergy laboratory sources only. The results of this $\mathrm{Cm}$ investigation have major implications for future studies of radioactive materiais. The technological impact of such measurements is expected to span nuclear solid waste characterization and storage, nonproliferation of nuclear weapons, and fundamental research of the electronic properties of the heaviest elements.

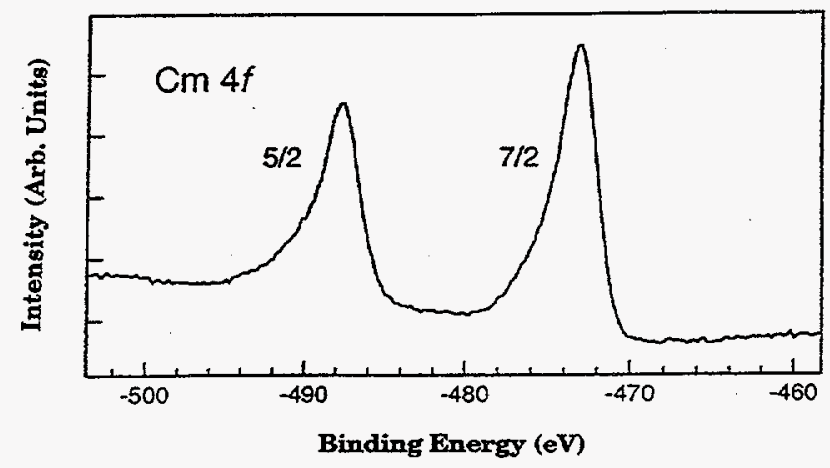

Figure 16-1. Curium $4 f$ spectrum obtained with $h v=1040 \mathrm{eV}$ excitation. An estimated 5 nanograms of curium oxide was illuminated by the $\sim 50 \mu \mathrm{m}$ diameter beam spot. (XBD 951-130)

\footnotetext{
This work was performed in part at ALS, which is operated by the Department of Energy, Division of Materials Sciences.
} 
17. Multinuclear NMR, Raman, EXAFS, and X-ray Diffraction Studies of Uranyl Carbonate Complexes in Near-neutral Aqueous Solution. X-ray Structure of $\left[\mathrm{C}\left(\mathrm{NH}_{2}\right)_{3}\right]_{6}\left[\left(\mathrm{UO}_{2}\right)_{3}\left(\mathrm{CO}_{3}\right)_{6}\right] \cdot 6.5 \mathrm{H}_{2} \mathrm{O}$ (Publication 28)

\section{P.G. Allen, J.J. Bucher, D.L. Clark, N.M. Edelstein, S.A.} Ekberg, J.W. Gohdes, E.A. Hudson, N. Kaltsoyannis, W.W. Lukens, M.P. Neu, P.D. Palmer, T. Reich, D.K. Shuh, C.D. Tail, and B.D. Zwick

${ }^{13} \mathrm{C}$ and ${ }^{17} \mathrm{O}$ NMR, and Raman spectroscopies were used to monitor the fractions of $\mathrm{UO}_{2}\left(\mathrm{CO}_{3}\right)_{3}{ }^{4-}(1)$ and $\left(\mathrm{UO}_{2}\right)_{3}\left(\mathrm{CO}_{3}\right)_{6}{ }^{6-}(2)$ in aqueous carbonate solutions as a function of $\mathrm{pH}$, ionic strength, carbonate concentration, uranium concentration, and temperature. The multinuclear NMR and Raman data are consistent with the formation of $\left(\mathrm{UO}_{2}\right)_{3}\left(\mathrm{CO}_{3}\right)_{6}{ }^{6-}$. The $\mathrm{pH}$ dependence of the ${ }^{13} \mathrm{C}$ NMR spectra was used to determine the equilibrium constant for the reaction $3 \mathrm{UO}_{2}\left(\mathrm{CO}_{3}\right)^{4-}+3 \mathrm{H}^{+} \leftrightarrow\left(\mathrm{UO}_{2}\right)_{3}\left(\mathrm{CO}_{3}\right)_{6}^{6-}+$ $3 \mathrm{HCO}_{3}{ }^{-}, \log \mathrm{K}=18.1( \pm 0.5)$ at $\mathrm{I}_{\mathrm{m}}=2.5 \mathrm{~m}$ and $25^{\circ} \mathrm{C}$, and corresponds to $\log \beta_{36}=55.6( \pm 0.5)$ for the reaction $3 \mathrm{UO}_{2}{ }^{2+}+6 \mathrm{CO}_{3}{ }^{2-} \leftrightarrow\left(\mathrm{UO}_{2}\right)_{3}\left(\mathrm{CO}_{3}\right)_{6}{ }^{6-}$ under the same conditions. Raman and IR spectra showed $v_{1}=831.6 \mathrm{~cm}^{-1}$ (Raman active) and $v_{3}=911 \mathrm{~cm}^{-1}$ (IR active) $U=O$ stretching bands for $1, v_{1}=812.5 \mathrm{~cm}^{-1}$ and $v_{3}=889 \mathrm{~cm}^{-1}$ for trimeric 2. EXAFS data from solid $\left[\mathrm{C}\left(\mathrm{NH}_{2}\right)_{3}\right]_{6}\left[\left(\mathrm{UO}_{2}\right)_{3}\right.$ $\left.\left(\mathrm{CO}_{3}\right)_{6}\right]$ and a solution of $\left(\mathrm{UO}_{2}\right)_{3}\left(\mathrm{CO}_{3}\right)_{6}{ }^{6-}$ suggest that the same uranium species is present in both the solid and solution states. Fourier transforms of the EXAFS spectra of both solid and solution samples revealed five well-resolved peaks corresponding to nearly identical near-neighbor distances for solid and solution samples of 2. Fitting of these peaks yields $\mathrm{U}-\mathrm{O}$ (uranyl) $=1.79, \mathrm{U}-\mathrm{O}$ (carbonate) $=$ 2.45 , U--C $=2.90$, (terminal carbonate) $=4.16$, and $\mathrm{U}-\mathrm{-U}=$ $4.91 \AA$ for the solid, and similar distances for the solution sample. The peak at $4.75 \AA$ in both Fourier transforms (uncorrected for phase shift) corresponds to the U--U interaction at $4.91 \AA$, a conclusion that is supported by the absence of this peak in the Fourier transform of the crystalline monomeric $\mathrm{K}_{4}\left[\left(\mathrm{UO}_{2}\right)\left(\mathrm{CO}_{3}\right)_{3}\right]$. Multiple scattering along the uranyl vector is believed to play a significant role in the EXAFS of all three systems. The EXAFS data are consistent with the trimeric uranyl carbonate species indicated by NMR spectroscopy. Single crystals of $\left[\mathrm{C}\left(\mathrm{NH}_{2}\right)_{3}\right]_{6}\left[\left(\mathrm{UO}_{2}\right)_{3}\left(\mathrm{CO}_{3}\right)_{6}\right] \cdot 6.5 \mathrm{H}_{2} \mathrm{O}$ were obtained from a solution that contained stoichiometric amounts of uranyl nitrate, guanidinium carbonate, and an excess of guanidinium nitrate at $\mathrm{pH} 6.5$ under a $\mathrm{CO}_{2}$ atmosphere. The solid state molecular structure of $\left[\mathrm{C}\left(\mathrm{NH}_{2}\right)_{3}\right]_{6}\left[\left(\mathrm{UO}_{2}\right)_{3}\left(\mathrm{CO}_{3}\right)_{6}\right] \cdot 6.5 \mathrm{H}_{2} \mathrm{O}$ contains a planar $\mathrm{D}_{3 \mathrm{~h}}$ trimetallic $\left(\mathrm{UO}_{2}\right)_{3}\left(\mathrm{CO}_{3}\right)_{6}{ }^{6-}$ anion (Figure 17-1), the structure that Åberg and coworkers originally proposed for the trimeric solution species. The trimetallic anion contains

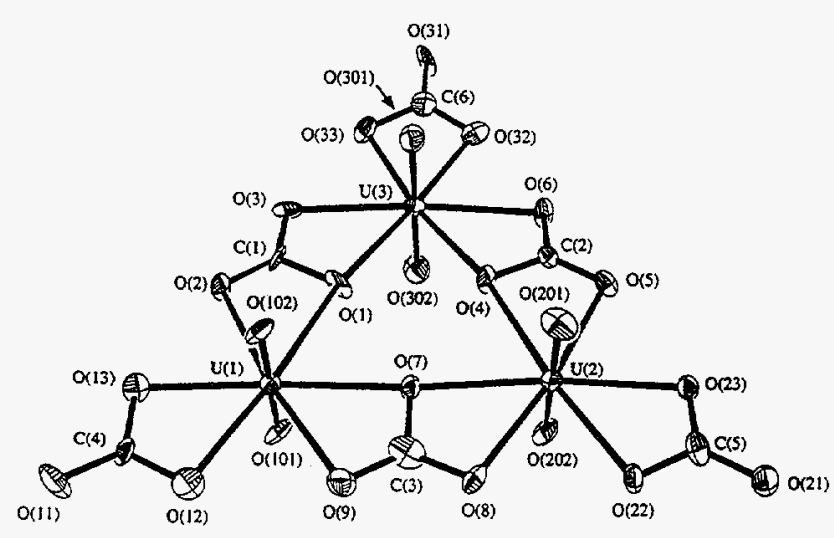

Figure 17-1. Crystal structure of the $\left(\mathrm{UO}_{2}\right)_{3}\left(\mathrm{CO}_{3}\right)_{6}{ }^{6-}$ anion. (XBD 951131)

three uranium atoms and all six carbonate ligands in the molecular plane with three uranyl oxygen atoms above, and three below the plane. Uranyl $\mathrm{U}=\mathrm{O}$ distances average 1.78(1) $\AA$, while $\mathrm{U}-\mathrm{O}$ distances to the carbonate oxygen atoms average 2.41(1) $\AA$ for terminal, and 2.48(1) $\AA$, for bridging ligands. Particularly significant is the average nonbonding $U--U$ distance of $4.97 \AA$ that compares favorably to the $4.91 \AA$ distance seen in the EXAFS analysis.

18. Electronic and Structural Investigations of Technetium Compounds by X-ray Absorption Spectroscopy (Publication 26)

\section{Almahamid, J.C. Bryan, J.J. Bucher, A.K. Burrell, N.M. Edelstein, E.A. Hudson, N. Kaltsoyannis, W.W. Lukens, H. Nitsche, T. Reich, and D.K. Shuh}

${ }^{99} \mathrm{Tc}$ is a by-product of nuclear fission, and its significant half-life $\left(\mathrm{t}_{1 / 2}=2.13 \times 10^{5}\right.$ years $)$ necessitates its consideration in the long-term disposal of nuclear waste. The principal Tc-containing component of nuclear wastes is $\mathrm{TcO}_{4}{ }^{-}$, and the propensity of $\mathrm{TcO}_{4}^{-}$to migrate in the geosphere has led to research aimed at the facile conversion of pertechnetate into other, less mobile compounds of ${ }^{99} \mathrm{Tc}$. An X-ray Absorption Near Edge Structure (XANES) spectroscopic evaluation of the efficacy of a process designed to reduce $\mathrm{TcO}_{4}{ }^{-}$to $\mathrm{TcO}_{2}$ in waste form cement was recently reported. The shapes and the chemical shifts of the $\mathrm{Tc} \mathrm{K}$ edge in $\mathrm{TcO}_{4}{ }^{-}, \mathrm{TcO}_{2}$, and $\mathrm{Tc}$ metal were employed to establish the oxidation state of $\mathrm{Tc}$. In this paper XANES is used to establish the chemical shifts of the technetium $\mathrm{K}$ edge in a range of compounds containing $\mathrm{Tc}$ in a variety of formal oxidation states as shown in Figure 18-1. The edge positions span $19.9 \mathrm{eV}$ 


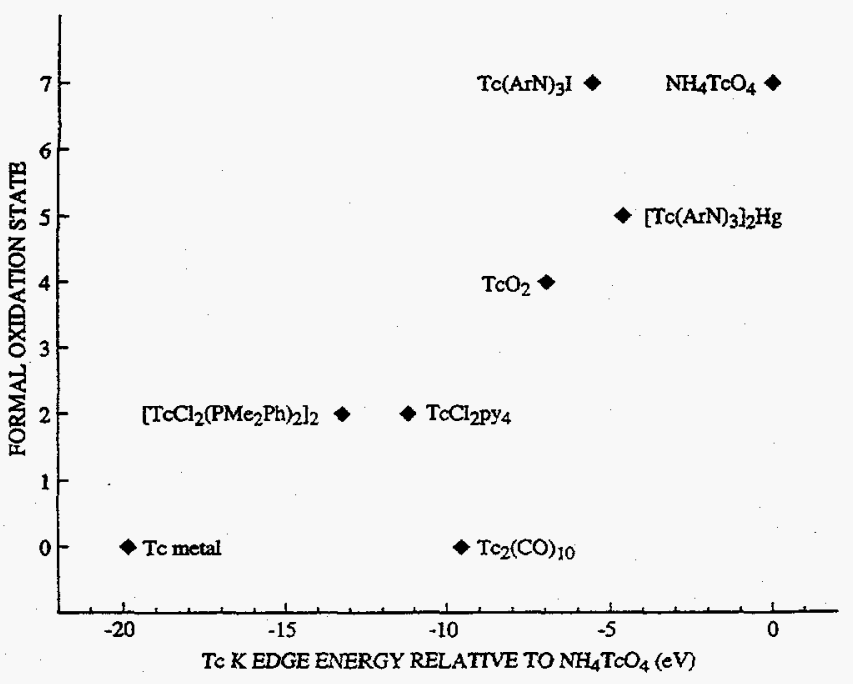

Figure 18-1. Chemical shifts of the Tc $\mathrm{K}$ edge vs. formal oxidation state for Tc metal, $\left[\mathrm{TcCl}_{2}\left[\left(\mathrm{PMe}_{2} \mathrm{Ph}\right)_{2}\right]_{2}, \mathrm{TcCl}_{2} \mathrm{Py}_{4}\left(\mathrm{py}=\mathrm{C}_{5} \mathrm{H}_{5} \mathrm{~N}\right), \mathrm{Tc}_{2}(\mathrm{CO})_{10}\right.$ $\mathrm{TcO}_{2}, \mathrm{Tc}(\mathrm{ArN})_{3} \mathrm{I}\left(\mathrm{ArN}=2,6-\mathrm{Pr}_{2}{ }_{2} \mathrm{C}_{6} \mathrm{H}_{3} \mathrm{~N}\right),\left[\mathrm{Tc}(\mathrm{ArN})_{3}\right]_{2} \mathrm{Hg}$ and $\mathrm{NH}_{4} \mathrm{TcO}_{4}$. (XBD 951-132)

from $\mathrm{Tc}$ metal to $\mathrm{NH}_{4} \mathrm{TcO}_{4}$. Strong correlation between chemical shift and formal oxidation state is observed. EXAFS spectroscopy of $\mathrm{Tc}_{2}(\mathrm{CO})_{10}$ indicates that multiple scattering along the $\mathrm{Tc}-\mathrm{C}-\mathrm{O}$ vector is more important than direct $\mathrm{Tc} \cdot . \mathrm{O}$ scattering. $\mathrm{TcO}_{2}$ is shown by EXAFS to possess a distorted rutile structure with a closest $\mathrm{Tc}-\mathrm{Tc}$ distance of $2.61 \AA$. This is rationalized in terms of the Goodenough model for bonding in transition metal dioxides.

\section{A XANES and EXAFS Investigation of the Speciation of Selenite Following Bacterial Metabolization (Publication 27)}

\section{B.B. Buchanan, J.J. Bucher, D.E. Carlson, N.M. Edelstein,} E.A. Hudson, N. Kaltsoyannis, T. Leighton, W. Lukens, H. Nitsche, T. Reich, K. Roberts, D.K. Shuh, P. Torretto, J. Woicik, W.-S. Yang, A. Yee, and B.C. Yee

The concentration and immobilization of hazardous materials by microbial agents offers great potential for use in environmental remediation technologies. In the case of selenium possible future applications of bioremediation technologies range from oil refinery waste streams containing selenite to agricultural drainage that has contaminated sites such as the Kesterson Reservoir in California. The aerobic soil bacterium, Bacillus subtilis, has been found to detoxify selenite although the metabolic mechanism is not fully understood. As in other cases, selenium oxyanions appear to be reduced to a red elemental

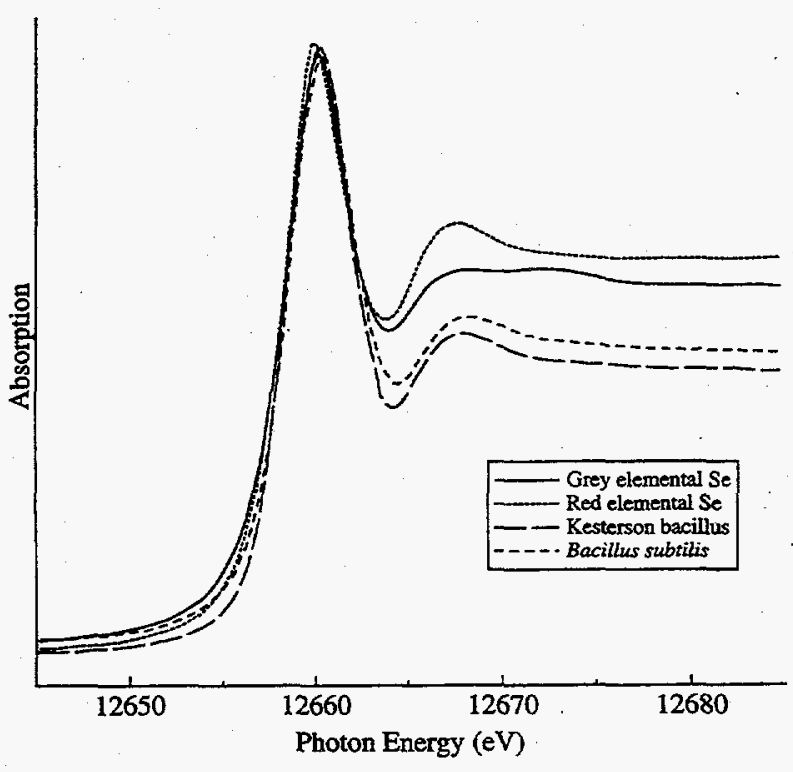

Figure 19-1. The selenium $K$ edge XANES spectra of amorphous red and grey selenium and from $B$. subtilis and the Kesterson bacillus after growth in a selenite medium. The spectra have been normalized to equivalent peak heights. (XBD 951-133)

form, although there is no direct in situ spectroscopic evidence to support this contention. This paper reports the XAS spectroscopic characterization of the oxidation state of selenium following microbial metabolism of selenite from aqueous solution. The common soil bacterium, Bacillus subtilis, can reductively metabolize selenite Se(IV). XANES spectroscopy demonstrates that both $B$. subtilis and an unidentified bacillus isolated from selenium contaminated soil reduce selenite to the red allotrope of elemental selenium. The red and grey forms of selenium are distinguished by their XANES spectra, (see Figure 19-1). EXAFS spectroscopy shows only one absorber-backscatter distance in both red and grey selenium, at $2.43 \AA$ and $2.44 \AA$, respectively.

\section{FY 1994 PUBLICATIONS AND REPORTS}

\section{Refereed Journals}

1. P.W. Durbin, B. Kullgren, J. Xu, and K.N. Raymond "In vivo Chelation of $\mathrm{Am}(\mathrm{III}), \mathrm{Pu}(\mathrm{IV}), \mathrm{Np}(\mathrm{V})$ and $\mathrm{U}(\mathrm{VI})$ in Mice by TREN-(Me-3,2-HOPO)," Rad. Prot. Dosimetry 53, 305 (1994); LBL-34569.

2. R. Cox, D.A. Buttry, P. Bonnesen and K.N. Raymond. "Measuring Trace Uranium," CHEMTECH 24, 18-2 (1994); LBL-36668. 
3. S.J. Franklin and K.N. Raymond, "Solution Structure and Dynamics of Lanthanide Complexes of the Macrocyclic Polyamino Carboxylate DTPA-dien. NMR Study and Crystal Structures of the Lanthanum(III) and Europium(III) Complexes," Inorg. Chem. 33, 5794 (1994); LBL-35200.

4. M. Weydert and R.A. Andersen, "New Synthetic Routes to Cyclopentadienyl-uranium (IV) Fluorides," ACS Symposium Series 555, 384 (1994).

5. P.G. Edwards, M. Weydert, M.A. Petrie, and R.A. Andersen, "Preparation and Synthetic Utility of $\mathrm{MCl}_{4}\left(\mathrm{Me}_{2} \mathrm{NCH}_{2} \mathrm{CH}_{2} \mathrm{NMe}_{2}\right)_{2}$ Where $\mathrm{M}$ is Uranium or Thorium," J. Alloys and Compounds 213/214, 11 (1994).

6. P. Thouvenot, S. Hubert and N. Edelstein, "Spectroscopic Study and Crystal Field Analysis of $\mathrm{Cm}^{3+}$ in the Cubic Symmetry Site of $\mathrm{ThO}_{2}$,' Phy. Rev. B 50, 9715 (1994).

7. K. Krämer, H.U. Güdel, G. Meyer, T. Heuer, N.M. Edelstein, B. Jun, L. Keller, P. Fischer, E. Zych, and J. Drozdzynski, "Synthesis, Crystal Structure, Magnetism, and Absorption Spectra of $A_{2} U X_{5}$ Type Halides $(A=K, R b$; $\mathrm{X}=\mathrm{Cl}, \mathrm{Br}, \mathrm{l})$," Z. Anorg. Allg. Chem. 620, 1339 (1994).

8. J.C. Krupa, E. Simoni, J. Sytsma, and N. Edelstein, "Optical Spectroscopic Studies of Uranyl Chloride $\mathrm{UO}_{2} \mathrm{CL}_{2}$," J. Alloys and Compounds 213, 471 (1994).

9. Y. Hinatsu and N. Edelstein, "Electron Paramagnetic Resonance Spectrum of $\mathrm{Pr}^{4+}$ in $\mathrm{BaCeO}_{3}$," J. Solid State Chem. 112(1), 53 (1994).

10. C. Qian, B. Wang, N. Edelstein, H. Reddman, C. Hagen, and H.-D. Amberger, "The Electronic Structure of Organometallic Complexes of the F-Elements XXXVI. Parameterization of the Crystal Field Splitting Patten of $\left.\mathrm{CH}_{3} \mathrm{OCH}_{2} \mathrm{CH}_{2} \mathrm{C}_{5} \mathrm{H}_{4}\right)^{3} \mathrm{Sm}^{\mathrm{III}}$," J. Alloys and Compounds 207, 87 (1994).

11. D.K. Shuh, L.J. Terminello, L.A. Boatner, M.M. Abraham, and D. Perry, "Characterization of Ce-Doped $\mathrm{LaPO}_{4}$ by Xray Absorption Spectroscopy," Mater. Res. Soc. Symp. Proc. 329, 91 (1994).

12. D.K. Shuh, C.W. Lo, J.A. Yarmoff, A. Santoni, L.J. Terminello, and F.R. McFeely, "Chlorine Chemisorption on Cleaved GaAs(110) and the Onset of Etching at Room Temperature," Surf. Sci. 303, 89 (1994).

13. V. Chakarian, D.K. Shuh, J.A. Yarmoff, H.-S. Tao, U. Diebold, B. Maschoff, N.D. Shinn, and T.E. Madey, "The Influence of Pre-adsorbed $\mathrm{K}$ on the Adsorption of $\mathrm{PF}_{3}$ on $\mathrm{Ru}(0001)$ Studied by Soft X-ray Photoelectron Spectroscopy," J. Chem. Phys. 100, 5301 (1994).

14. T.D. Durbin, J.R. Lince, S.V. Didziulis, D.K. Shuh, and J.A Yarmoff, "Soft X-ray Photoelectron Spectroscopy Study of the Interaction of $\mathrm{Cr}$ with $\mathrm{MoS}_{2}(0001)$ Surface," Surf. Sci. 302, 314 (1994).

15. T.D. Durbin, W.C. Simpson, V. Chakarian, D.K. Shuh, and J.A. Yarmoff, "Stimulated Desorption of $\mathrm{Cl}^{+}$and the Chemisorption of $\mathrm{Cl}_{2}$ on $\mathrm{Si}(111)-7 \times 7$ and $\mathrm{Si}(100)-2 \times 1$," Surf. Sci. 316, 257 (1994).

\section{LBL Reports}

16. K.N. Raymond and J. $\mathrm{Xu}$, "Siderophore-based Hydroxypyridonate Sequestering Agents" in The
Development of Iron Chelators for Clinical Use, R.J. Bergeron and G.M. Brittenham, Eds. (CRC Press, Inc.: Boca Baton, FL, 1994), p. 307; LBL-36670.

17. J.L. Stewart and R.A. Andersen, "Crystal Structures of $\left[\left(\mathrm{Me}{ }_{3} \mathrm{Si}\right)_{2} \mathrm{~N}_{4} \mathrm{U}_{2}[\mu-\mathrm{N}(\mathrm{H})(\text { mesityl })]_{2}\right.$ and $\left[\left(\mathrm{Me}_{3} \mathrm{Si}\right)_{2} \mathrm{~N}\right]_{4}$ $\mathrm{U}_{2}[\mu-\mathrm{N} \text { (p-tolyl) }]_{2}$; Compounds with Asymmetrically Bridging Primary Amide and Imide Groups," LBL-27796.

18. M.R. Smith, III, P.T. Matsunaga, and R.A. Andersen, "Preparation of Monomeric $\left(\mathrm{Me}_{5} \mathrm{C}_{5}\right)_{2} \mathrm{VO}$ and $\left(\mathrm{Me}_{5} \mathrm{C}_{5}\right)_{2} \mathrm{Ti}(\mathrm{O})(\mathrm{L})$ and Their Decomposition to $\left(\mathrm{Me}_{5} \mathrm{C}_{5}\right)_{4} \mathrm{M}_{4}(\mu-\mathrm{O})_{6}$," LBL-33610.

19. W.W. Lukens and R.A. Andersen, "Dinuclear Titanium (III) Complexes; Exchange Coupling in $\left(\mathrm{Cp}_{2} \mathrm{Ti}\right)_{2}(\mu-\mathrm{O})$," LBL36672.

20. E. Brady, W. Lukens, J.R. Telford, and G. Mitchell, "Bis(pentamethylcyclopentadienyl)-titanium Amide," LBL34892.

21. W. Lukens, J.R. Telford, and G. Mitchell, "Tris(trimethylsilylcyclopentadienyl)zirconium Chloride," LBL-34891.

22. D.J. Schwartz and R.A. Andersen, "Reversible Formation of $\left[\mathrm{P}_{2} \mathrm{Pt} \mathrm{H}\right]_{2} \mathrm{Pt}(\mathrm{I})$ Complexes from cis- $\mathrm{P}_{2} \mathrm{Pt} \mathrm{H} \mathrm{H}_{2}$ Complexes, where $\mathrm{P}_{2}$ is a Chelating Phosphine," LBL-36674.

23. P. Selg, H.H. Brintzinger, R.A. Andersen, and I.T. Horvath, "Coordination of $\mathrm{CO}$ to the Alkaline-Earth Metallocene $\left(\mathrm{Me}_{5} \mathrm{C}_{5}\right)_{2} \mathrm{Ca}$," LBL-36671.

24. N.M. Edelstein, "Comparison of the Electronic Structure of the Lanthanides and Actinides," LBL-36124.

25. W.K. Kot and N.M. Edelstein, "The Synthesis and Electronic Structure of $\mathrm{Pa}\left(\mathrm{BH}_{3} \mathrm{CH}_{3}\right)_{4}$," LBL-36673.

26. I. Almahamid, J.C. Bryan, J. J. Bucher, A.K. Burrell,N.M. Edelstein, E.A. Hudson, N. Kaltsoyannis, W.W. Lukens, H. Nitsche, T. Reich, and D.K. Shuh, "Electronic and Structural Investigations of Technetium Compounds by $X$ ray Absorption Spectroscopy," LBL-36432.

27. B.B. Buchanan, J.J. Bucher, D.E. Carlson, N.M. Edelstein, E.A. Hudson, N. Kaltsoyannis, T. Leighton, W. Lukens, $H$. Nitsche, T. Reich, K. Roberts, D.K. Shuh, P. Torretto, J. Woicik, W-S. Yang, A. Yee, and B.C. Yee, "A XANES and EXAFS Investigation of the Speciation of Selenite Following Bacterial Metabolization," LBL-36690.

28. P.G. Allen, J.J. Bucher, D.L. Clark, N.M. Edelstein, S.A. Ekberg, J.W. Gohdes, E.A. Hudson, N. Kaltsoyannis, W.W. Lukens, M.P. Neu, P.D. Palmer, T. Reich, D.K. Shuh, C.D. Tait, and B.D. Zwick, "Multinuclear NMR, Raman, EXAFS, and X-ray Diffraction Studies of Uranyl Carbonate Complexes in Near-neutral Aqueous Solution. X-ray Structure of $\left[\mathrm{C}\left(\mathrm{NH}_{2}\right)_{3}\right]_{6}\left[\left(\mathrm{UO}_{2}\right)_{3}\left(\mathrm{CO}_{3}\right)_{6}\right] \cdot 6.5 \mathrm{H}_{2} \mathrm{O}$," LBL36676.

29. D. Whisenhunt Jr., "Synthesis and Evaluation of Chelating Agents and Resins Specific for the Actinides,." Ph.D Thesis; LBL-36677.

30. D.K. Shuh, N. Kaitsoyannis, J.J. Bucher, N. M. Edelstein, S.B. Clark, H. Nitsche, T. Reich, E.A. Hudson, I. Almahamid, P. Torretto, W. Lukens, K. Roberts, B. C. Yee, D.E. Carlson, A. Yee, B.B. Buchanan, T. Leighton, W.-S. Yang, and J.C. Bryan, "Environmental Applications of XANES: Speciation of Tc in Cement After Chemical 
Treatment and Se After Bacterial Uptake," Mater. Res. Soc. Symp. Proc. (in press); LBL-35411.

31. J. D. Denlinger, E. Rotenberg, T. Warwick, G. Visser, J. Nordgren, J.-H. Guo, P. Skytt, S.D. Kevan, K.S. McCutcheon, J.G. Tobin, D.K. Shuh, J.J. Bucher, N.M. Edelstein, and B.P. Tonner, "First Results From the SpectroMicroscopy Beamline at the ALS," Rev. Sci. Instrum. (in press); LBL-36713.

\section{Other Publications}

32. J.A. Carlisle, L. J. Terminello, A. V. Hamsa, E.A. Hudson, E.L. Shirley, F. J. Himpsel, D.A. Lapiano-Smith, J. J. Jia, T.A. Calcott, R.C.C. Perera, D.K. Shuh, S.G. Louie, J. Stöhr, M.G. Samant, and D.L. Ederer, "Occupied and Unoccupied Orbitals of $\mathrm{C}_{60}$ and $\mathrm{C}_{70}$," Mat. Res. Soc. Symp.Proc., "Novel Forms of Carbon," (in press).

33. J.A. Carlisle, L.J. Terminello, A.V. Hamsa, E.A. Hudson, E.L. Shirley, F.J. Himpsel, D.A. Lapiano-Smith, J.J. Jia, T. A. Calcott, R.C.C. Perera, D. K. Shuh, S.G. Louie, J. Stöhr, M.G. Samant, and D.L. Ederer, "Occupied and Unoccupied Orbitals of $\mathrm{C}_{60}$ and $\mathrm{C}_{70}$," Mol. Cryst. (in press).

\section{Invited Talks}

34. Z. Hou, X. Jide, K.N. Raymond, D.W. Whisenhunt Jr., M.P. Neu, D.C. Hoffman, "Derivatives of Natural Complexing Agents for Removal of Pu(IV) from Waste Streams," ESPIP Technical Exchange Workshop, January 10-11, 1994.

35. D. Whisenhunt, "Current Status of Actinide Specific Chelation: Mammalian Decorporation," Symposium on Environmental Fate and Dynamics of Inorganics, American Chemical Society Meeting, San Diego, March 13-16, 1994.

36. S.J. Franklin and K.N. Raymond, "Solution Structure and Dynamics of Macrocyclic Lanthanide DTPA-Bisamides," XIX International Symposium on Macrocyclic Chemistry, Lawrence, KS, June 12-17, 1994.

37. J.R. Telford, K.N. Raymond, and B.R. Byers, "Isolation and Characterization of a New Series of Siderophores- the Amonabactins," NATO Advanced Study Institute on "Bioinorganic Chemistry: An Inorganic Perspective of Life," June 5-17, 1994.

38. P.W. Durbin and K.N. Raymond, "New Actinide-Chelating Agents: An Overview," Conference on Chelating Agents and Metal Toxicity, National Institute of Health Sciences (NIEHS), Research Triangle Park, NC, September 22-23, 1994.

39. R.A. Andersen, "Early Metal and Lanthanide Metal," American Chemical Society Meeting, San Diego, CA March 14, 1994.

40. R.A. Andersen, "Early Metal and Lanthanide Metal," University of Utah, Salt Lake City, UT, May 16, 1994.

41. R.A. Andersen, "Early Metal and Lanthanide Metal," Utah State University, Logan, UT, May 17, 1994.

42. R.A. Andersen, Max Planck Institute for Radiation Chemistry, Mülheim, Germany, November 16, 1994.

43. R.A. Andersen, "Early Metal and Lanthanide Metal," University of Göttingen, Germany, December 5, 1994.
44. R.A. Andersen, "Early Metal and Lanthanide Metal," University of Breman, Germany, December 7, 1994.

45. R.A. Andersen, "Early Metal and Lanthanide Metal," University of Hamburg, Germany, December 13, 1994.

46. R.A. Andersen, "Early Metal and Lanthanide Metal," Technical University-Berlin, Germany, December 19, 1994.

47. N. Edelstein, "Comparison of the Electronic Structure of the Lanthanides and Actinides," First Workshop on Comparative Science of the f-Elements, Baden-Baden, Germany, July 28-30, 1994.

48. N. Edelstein, "Optical Spectroscopy of f-Element Compounds," Workshop on Relativistic Effects in HeavyElement Chemistry and Physics: Electronic Structure Methods for Lanthanides and Actinides, Helsinki, Finland, August 8, 1994.

49. D.K. Shuh, "Applications of Synchrotron Radiation Techniques to Environmental Problems," Department of Physics Colloquia, University of Missouri-Rolla, Rolla, MO, September 29, 1994.

50. D.K. Shuh, "Applications of Synchrotron Radiation to Chemical Issues in the Environmental Sciences," ACS National Symposium, Synchrotron Radiation Techniques in Industrial Research, Washington D.C., August 24, 1994.

\section{Invited Lectures}

51. K.N. Raymond, "The Design, Synthesis and Evaluation of Actinide-Sequestering Agents,” First DOE/BES Separations Research Conference, Seattle, WA, October 14-15, 1994.

52. K.N. Raymond, "Characterization and Properties of Amonabactins: Fresh-water Siderophores," Gordon Research Conference on Marine Bioinorganic Chemistry, Ventura, CA, January 24-28, 1994.

53. K.N. Raymond, "Metal-Ion-Selective and Stereognostic Coordination Chemistry," Seminar, Colorado State University, Denver, CO, February 22, 1994.

54. K.N. Raymond, "Metal-Ion-Selective and Stereognostic Coordination Chemistry," Seminar, University of Colorado, Boulder, CO, February 24, 1994.

55. K.N. Raymond, "Metal-Ion-Selective and Stereognostic Coordination Chemistry," Seminar, University of Wyoming, Laramie, WY, February 25, 1994.

56. K.N. Raymond, "Stereognostic Coordination Chemistry," Bürgenstock Meeting, Switzerland, May 1-8, 1994.

57. K.N. Raymond, "Bioinorganic Chemistry: An Inorganic Perspective of Life," 1) "The Coordination Chemistry of Iron in Biological Transport and Storage;" 2) Siderophoremediated Iron Transport in Microbes," NATO Advanced Study Institute, June 5-17, 1994.

58. K.N. Raymond, "Siderophore Iron Coordination Chemistry and Bacterial Disease," Keynote Lecturer, Inorganic Biochemistry Summer Workshop, Athens, GA, August 9 , 1994.

59. K.N. Raymond, "Stereognostic Coordination Chemistry of Lead: New Chelating Agents," Conference on Chelating Agents and Metal Toxicity, National Institute of Health Sciences (NIEHS), Research Triangle Park, NC, September 22-23 1994. 


\section{CHEMICAL ENGINEERING SCIENCES}

\section{Molecular Thermodynamics for Phase Equilibria in Mixtures*}

John M. Prausnitz, Investigator

\section{INTRODUCTION}

Molecular thermodynamics provides a useful tool for calculating phase equilibria in mixtures from a minimum of experimental information. Such equilibria are required for design of separation operations and, frequently, for design of new chemical products (e.g., polymeric alloys or drugdelivery systems). Molecular thermodynamics is an engineering-oriented subject based on classical and statistical thermodynamics, and on models from physical chemistry and chemical physics.

For research reported here, the ultimate goal is quantitative prediction of phase equilibria in a variety of mixtures encountered in the chemical and related industries, including those using biotechnology. Prediction is based on insightful correlation of experimental data; such correlation can only follow from interpretation of data through theoretically based models. Therefore, the essence of research reported here is to establish meaningful models that can generalize limited experimental information toward correlation and quantitative prediction or phase behavior in mixtures.

Attention is given to three topics: polymer/solvent and polymer/polymer mixtures; aqueous solutions of proteins containing concentrated salts; and hydrogels in contact with solute-containing water.

1. Molecular Thermodynamics of Polymer Solutions and Blends

Vapor-Liquid Equilibria of Copolymer/Solvent and Homopolymer/Solvent Binaries: New Experimental Data and Their Correlation

\section{R.B. Gupta and J.M. Prausnitz}

Sixty-six data-sets for vapor-liquid equilibria (VLE) in polymer/solvent binary solutions have been obtained using

*This work was supported by the Director, Office of Energy Research, Office of Basic Energy Sciences, Chemical Sciences Division, of the U.S. Department of Energy under Contract No. DE-AC03-76SF00098. a gravimetric sorption technique. Effect of copolymer composition on VLE behavior was examined. Solvents studied were: acetone, acetonitrile, n-butanol, 1,2dichloroethane, chloroform, cyclohexane, n-hexane, methanol, octane, pentane, and toluene. Copolymers studied were: poly(acrylonitrile-co-butadiene), poly(styrene-coacrylonitrile), poly(styrene-co-butadiene), poly(styrene-cobutyl methacrylate), poly(vinyl acetate-co-ethylene), and poly(vinyl acetate-co-vinyl chloride). All the copolymers studied were random copolymers. Some homopolymers were also studied: polyacrylonitrile, polybutadiene, poly(butyl methacrylate), poly(ethylene oxide), polystyrene, and poly(vinyl acetate).

"Expected" non-ideal behavior has been observed for systems such as cyclohexane/poly(ethylene-co-vinyl acetate) and chloroform/poly(styrene-co-butyl methacrylate). However, strong non-ideal behavior has been observed for systems containing hydrocarbons and poly(butadiene-co-acrylonitrile). The perturbed hardsphere-chain (PHSC) equation of state for mixtures of heteronuclear chains was used to represent vapor-liquid equilibria of these copolymer solutions.

Liquid-Liquid Equilibria for Copolymer Mixtures from a Perturbed Hard-Sphere-Chain Equation of State

\section{T. Hino, Y. Song, and J.M. Prausnitz}

A perturbed hard-sphere-chain (PHSC) equation of state for real copolymer mixtures is based on a modified form of Chiew's equation of state for athermal mixtures of heteronuclear hard-sphere chains. The PHSC equation of state includes a van-der-Waals perturbation whose parameters are related to the intermolecular potential as suggested by Song and Mason. In the present model, sequence distribution in a polymer is introduced only into the hard-sphere-chain reference state; attractive forces are averaged, independent of sequence distribution. Theoretical coexistence curves and miscibility maps were computed for binary random copolymer mixtures containing two or three kinds of segments. The PHSC equation of state can predict simultaneous occurrence of a lower critical solution temperature and an upper critical solution temperature in the temperature-composition phase diagram of high- 
molecular-weight copolymer blends. Theoretical and experimental coexistence curves and miscibility maps show good agreement for systems containing two kinds of segments.

A Perturbed Hard-Sphere-Chain Equation of State for Normal Fluids and Polymers (Publication 8)

\section{Y. Song, S.M. Lambert, and J.M. Prausnitz}

A PHSC equation of state is presented that uses the modified Chiew equation of state for hard sphere chains as the reference term, a van der Waals type perturbation term and the Song-Mason method to relate equation-of-state parameters to the intermolecular potential. The PHSC equation requires three parameters to describe a pure fluid: the number of effective hard-spheres per molecule, the segmental diameter, and the intermolecular potential-well depth between an unbonded pair of segments. The PHSC equation reproduces the thermodynamic properties of a wide variety of normal fluids including polymers. Regressed parameters are related to characteristic molecular properties calculated from group contributions based on chemical structure.

Liquid-Liquid Phase Diagrams for Binary Polymer Solutions from a Perturbed Hard-Sphere-Chain Equation of State (Publication 14)

\section{Y. Song, S.M. Lambert, and J.M. Prausnitz}

The PHSC equation of state for multicomponent mixtures is presented as a generalization from the equation of state for pure fluids. The reference term, based on Chiew's equation of state for hard-sphere chains, requires no mixing rules. Only the attractive perturbation requires van der Waals one-fluid mixing rules. Cross parameters needed in the perturbation are obtained using pure-fluid parameters and simple combining rules. Simplifying physical assumptions are given to reduce the perturbation term to the Flory $\chi$ parameter. Specific interactions are included by adapting the model of ten Brinke and Karasz. Model calculations for binary mixtures demonstrate that the PHSC equation can predict lower critical solution temperatures, upper critical solution temperatures and closed partial-miscibility loops. Special attention is given to the effects of polymer molecular weight, pressure, differences in segment size, and differences in segment interaction energy.
2. Precipitation of Proteins in Aqueous Solutions Using Salts

Molecular Thermodynamics for Salt-Induced Protein Precipitation

Y.C. Chiew, D.E. Kuehner, H.W. Blanch, and J.M. Prausnitz

A molecular-thermodynamic model is developed for salt-induced protein precipitation; the model considers an aqueous solution of globular protein molecules as a pseudo one-component system containing macroions that interact through Coulombic repulsion, dispersion attraction, hydrophobic interactions, and forces arising from ionexcluded volume. Forces from ion-excluded volume take into account formation of ion pairs and ionic clusters at high salt concentrations; they are calculated in the context of the Percus-Yevick integral-equation theory. Hydrophobic interactions between exposed non-polar amino-acid residues on the surfaces of the protein molecules are modeled as short-range, attractive interactions between "spherical caps" on the surfaces of the protein polyions. An equation of state is derived using perturbation theory. From this equation of state we calculate liquid-liquid equilibria, i.e., equilibrium between an aqueous phase dilute in protein and another aqueous phase rich in protein; the latter represents "precipitated" protein. In the equation of state, center-to-center, spherically symmetric macroion-macroion interactions are described by the random-phase approximation, while the orientation-dependent short-range hydrophobic interaction is incorporated through the perturbation theory of associating fluids. The results obtained here suggest that either ion-excluded-volume or hydrophobic-bonding effects can precipitate proteins in aqueous solutions with high salt concentrations.

Salting Out Aqueous Proteins: Phase Equilibria and Intermolecular Potentials

\section{C.J. Coen, H.W. Blanch, and J.M. Prausnitz}

Salting-out phase equilibria are reported for lysozyme and $\alpha$-chymotrypsin from concentrated ammonium-sulfate solutions. Supernatant and dense-phase protein concentrations and the resulting protein partition coefficients are given as a function of solution $\mathrm{pH}$ and ionic strength. Phase equilibria with a trivalent salt (sodium citrate) confirm that ionic strength, rather than salt concentration, is the appropriate variable describing phase equilibria. Salting-out behavior of an aqueous mixture of 
lysozyme and $\alpha$-chymotrypsin is independent of the presence of the other protein.

Parameters for a molecular-thermodynamic description of salting-out behavior are obtained from low-angle laserlight scattering (LALLS). Osmotic second virial coefficients from LALLS are reported over a range of $\mathrm{pH}$ for dilute chymotrypsin concentrations in aqueous electrolyte solutions at 0.01 and $1.0 \mathrm{M}$ ionic strengths. Effective Hamaker constants, regressed from experimental osmotic second virial coefficients, are determined for models of the protein-protein potential of mean force. In addition to excluded volume, dispersion and shielded charge-charge potentials, our description of protein-protein interactions includes attractive charge-dipole and dipoledipole potentials as well as an osmotic-attraction potential that becomes important at high salt concentrations. Protein dipole-dipole potentials are required to account for the observed $\mathrm{pH}$ dependence of osmotic second virial coefficients, especially at low ionic strength.

\section{Thermodynamic Properties of Hydrogels}

\section{Phase Equilibria for Aqueous Protein/Polyelectro- lyte Gel Systems}

\section{A.P. Sassi, H.W. Blanch, and J.M. Prausnitz}

A molecular-thermodynamic analysis is directed toward predicting the partitioning of aqueous proteins into charged hydrogels. This analysis takes into account size exclusion by the network, electrostatic interactions, and the osmotic-pressure difference between a hydrogel and its surrounding solution. Electrostatic interactions in the polyelectrolyte gel can be described by Debye-Hückel theory, or the Mean Spherical Approximation, or Katchalsky's cell model for polyelectrolyte solutions. The cell model gives best agreement with experimental partition coefficients for cytochrome $c$. The quasi-electrostatic potential difference between a gel and its surrounding solution demonstrates how the electrostatic contribution to the protein partition coefficient depends on protein charge, gel-charge density, and solution ionic strength. Finally, a qualitative guide is presented for design of a polyelectrolyte gel such that it exhibits specified swelling and partitioning properties.
Partitioning of Proteins and Small Biomolecules in Temperature-and $\mathrm{pH}$-Sensitive Hydrogels

\section{A.P. Sassi, A.J. Shaw, S.M. Han, H.W. Blanch, and J.M. Prausnitz}

Experimental partition coefficients were measured for the distribution of selected proteins and small biomolecules between aqueous, buffered solutions and hydrogels. Temperature-sensitive hydrogels were prepared by polymerizing $\mathrm{N}$-isopropylacrylamide (NIPA) alone and by co-polymerizing NIPA and a charged monomer. Temperature- and $\mathrm{pH}$-sensitive hydrogels were prepared by co-polymerizing NIPA with weakly acidic and/or weakly basic monomers. The effects of temperature, $\mathrm{pH}$ and ionic strength on partitioning were investigated. In general, protein partition coefficients ranged from 0 to 10 in buffers of $0.1 \mathrm{M}$ ionic strength. $\mathrm{pH}$ had a significant effect on the partition coefficient for a protein into a weakly ionizable polyelectrolyte gel even at this ionic strength.

Partitioning of Hexavalent Chromium in Temperature-Sensitive, Polyelectrolyte Hydrogels

\section{A.P. Sassi, D. Freed, H.W. Blanch, and J.M. Prausnitz}

Partitioning data for hexavalent chromium (Cr(VI)) are presented for systems where thermally sensitive poly-Nisopropylacrylamide (NIPA)-based hydrogels are in contact with aqueous solutions of potassium dichromate $\left(\mathrm{K}_{2} \mathrm{Or}_{2} \mathrm{O}_{7}\right)$. The poly-NIPA hydrogels contain $0-3 \%$ quaternized amine comonomer. Experimental results are given for the effect of gel charge, ionic strength, and temperature on the partitioning of $\mathrm{Cr}(\mathrm{VI})$ into these NIPAcopolymer gels. Swelling equilibria in aqueous $\mathrm{K}_{2} \mathrm{Cr}_{2} \mathrm{O}_{7}$ solutions are compared to swelling in aqueous solutions of sodium chloride. Experimental partitioning data as a function of ionic strength and gel charge are compared with predictions based on several theoretical models for mixed electrolyte solutions. Predictions based on the cell model for polyelectrolyte solutions agree best with experimental results. Calculated results are in semi-quantitative agreement with experimental data for the effects of solution ionic strength and gel charge on $\mathrm{Cr}(\mathrm{VI})$ partitioning and in qualitative agreement for the effect of temperature. 
Sorption of Lysozyme by HEMA Copolymer Hydrogels

\section{A.P. Sassi, S.H. Lee, Y.H. Park, H.W. Blanch, and J.M. Prausnitz}

Sorption of lysozyme by 2-hydroxyethylmethacrylate (HEMA) copolymer hydrogels has been studied as a function of $\mathrm{pH}$ and gel composition. Three types of HEMA gels were synthesized: neutral (HEMA), acidic (HEMA + acrylic acid), and basic (HEMA + dimethylaminoethylmethacrylate). Each gel was synthesized at four initial volume fractions to obtain different equilibrium swelling ratios and microstructures. Sorption as a function of time was measured for each gel at

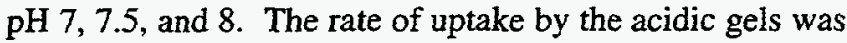
more rapid than that by the neutral gels: to sorb $90 \%$ of the protein required only one hour for the acidic gels but 15 days for neutral gels. Lysozyme did not adsorb or partition into the basic gels. The fractional approach to equilibrium was most rapid for the more swollen gels, and the effect of $\mathrm{pH}$ was small. The results reported here may be useful for rational design of new biomaterials where it is desirable to know the relative magnitudes of the effects of gel composition and synthesis, and solution $\mathrm{pH}$ on protein sorption.

\section{FY 1994 PUBLICATIONS AND REPORTS}

\section{Refereed Journals}

1. T. Hino, S.M. Lambert, D.S. Soane, and J.M. Prausnitz, "Miscibilities in Binary Copolymer Systems," Polymer 34 (22), 4756 (1993).

2 Y. Hu, X. Ying, D.T. Wu, and J.M. Prausnitz, "LiquidLiquid Equilibria for Solutions of Polydisperse Polymers," Macromolecules 26 (25), 6817 (1993).

3. J.M. Prausnitz, "Rediscovering the Relevance of Chemical Engineering," Chemtech 23,12 (1993).

4. T. Hauschild and J.M. Prausnitz, "Monte-Carlo Calculations for Methane and Argon Over a Wide Range of Density and Temperature Including the Two-Phase Vapor-Liquid Region," Mol. Simul. 11, 177 (1993).

5. Y. Song, S.M. Lambert, and J.M. Prausnitz, "Equation of State for Mixtures of Hard-Sphere Chains Including Copolymers," Macromolecules 27, 441 (1994).

6. J.P. Baker, L.H. Hong, H.W. Blanch, and J.M. Prausnitz, "Effect of Initial Total Monomer Concentration on the Swelling Behavior of Cationic Acrylamide-Based Hydrogeis," Macromolecules 27, 1446 (1994).

7. H. Zerres and J.M. Prausnitz, "Thermodynamics of Phase Equilibria in Aqueous-Organic Systems with Salt," AIChE J. 40, 676 (1994).
8. Y. Song, S.M. Lambert, and J.M. Prausnitz, "A Perturbed Hard-Sphere-Chain Equation of State for Normal Fluids and Polymers," IEC Res. 33, 1047 (1994).

9. J.P. Baker, H.W. Blanch, and J.M. Prausnitz, "Equilibrium Swelling Properties of Weakly-Ionizable 2-Hydroxyethyl Methacrylate (HEMA)-Based Hydrogels," J. App. Polym. Sci. 52, 783 (1994).

10. A. Anderko and J.M. Prausnitz, "On the Relationship Between the Equilibrium Constants of Consecutive Association Reactions," Fluid Phase Equilib. 95, 59 (1994).

11. Y. Hu, H. Liu, and J.M. Prausnitz, "A Model-Free Method for Calculating Vapor-Liquid Equilibria for Multicomponent Systems from Total-Pressure or BoilingPoint Data," Fluid Phase Equilib. 95, 73 (1994).

12. M. Kremer, E. Pothmann, T. Rössler, J.P. Baker, A. Yee, H.W. Blanch, and J.M. Prausnitz, "Pore-Size Distributions of Cationic Polyacrylamide Hydrogels Varying in Initial Monomer concentration and Cross-Linker/Monomer Ratio," Macromolecules 27, 2965 (1994).

13. Y. Hu, X. Ying, D.T. Wu, and J.M. Prausnitz, "LiquidLiquid Equilibria for Solutions of Polydisperse Polymers. Continuous Thermodynamics for the Lattice-Fluid Model," Fluid Phase Equilib. 98, 113 (1994).

14. Y. Song, S.M. Lambert, and J.M. Prausnitz, "Liquid-Liquid Phase Diagrams for Binary Polymer Solutions from a Perturbed Hard-Sphere-Chain Equation of State," Chem. Eng. Sci. 49 (17), 2765 (1994).

\section{Other Publications}

15. M.H. Imbacuán, "Osmotic-Pressure Measurements of Aqueous Solutions of Ovalbumin and Alphachymotrypsin," (jointly with Prof. H.W. Blanch), M.S. Thesis, University of California, Berkeley, CA, May 1994.

16. W.L. Jaecksch, "Liquid/Liquid/Solid Equilibria of Sodium Sulfate, Sodium Sulfite and Sodium carbonate; Water; and Acetone, 2-Propanol, and t-Butanol," (jointly with Prof. S. Lynn), M.S. Thesis, University of California, Berkeley, CA, December 1993.

17. A.L. Lopes Schiozer, "Quaternary Liquid/Liquid Equilibria of Sodium Sulfate, Sodium Sulfite, and Water with Two Solvents: Acetone and 2-Propanol," (jointly with Prof. S. Lynn), M.S. Thesis, University of California, Berkeley, CA, May 1994.

18. J.P. Baker, "Equilibrium Swelling Properties of Ionized Hydrogels," (jointly with Prof. H.W. Blanch), Ph.D. Thesis, University of California, Berkeley, CA, December 1993.

\section{LBL Reports}

19. C. Lira-Galeana, A. Firoozabadi, and J.M. Prausnitz, "Computation of Compositional Grading in Hydrocarbon Reservoirs," Fluid Phase Equilib. (submitted); LBL-34807.

20. D.H. Walther, G.H. Sin, H.W. Blanch, and J.M. Prausnitz, "Pore-Size Distributions of Cationic 2-Hydroxyethyl Methacrylate (HEMA) Hydrogels," J. Macromolecular Sci.Chem. Phys. Rev. in Macromolecular Chem. \& Phys. (in press); LBL-34808. 
21. D.H. Walther, H.W. Blanch, and J.M. Prausnitz, "Pore-Size Distributions of N-isopropylacrylamide (NIPA) Hydrogels," LBL-34875.

22. T. Hino, Y. Song, and J.M. Prausnitz, "Liquid-Liquid Equilibria for Copolymer Mixtures From a Perturbed HardSphere-Chain Equation of State," Macromolecules (in press); LBL-35398.

23. J. von Schnitzler and J.M. Prausnitz, "A New AssociationModel for Binary VLE of Alcohol / Hydrocarbon and Amine / Hydrocarbon Mixtures," LBL-35431.

24. J. von Schnitzler and J.M. Prausnitz, "A Classical Model for Closed-Loop Diagrams of Binary Liquid Mixtures," LBL35432.

25. C.J. Coen, H.W. Blanch, and J.M. Prausnitz, "Proteins in Aqueous Electrolyte Solutions: Measurements of SaltingOut Phase Equilibria and Comments on Protein Intermolecular Potentials," AIChE J. (in press); LBL-35480.

26. A.L. Schiozer, "Quaternary Liquid/Liquid Equilibria of Sodium Sulfate, Sodium Sulfite and Water with Two Solvents: Acetone and 2-Propanol," LBL-35481.

27. Y.C. Chiew, D. Kuehner, H.W. Blanch, and J.M. Prausnitz, "Molecular Thermodynamics for Salt-Induced Protein Precipitation," AIChE J. (in press); LBL-35487.

28. O. Pfohl, T. Hino and J.M. Prausnitz, "On the Temperature Calibration of a Thermooptical Apparatus," Fluid Phase Equilib. (submitted); LBL-35588.

29. J.M. Prausnitz, "Some New Frontiers in Chemical Engineering Thermodynamics," Fluid Phase Equilib. (submitted); LBL-35664.

\section{Invited Talks}

30. J.M. Prausnitz, "Against Babelism: Diversity, Hermeneutics and Legitimacy. Aspect of Engineering for the End of This Century" and "Molecular Thermodynamics for Chemical Process and Product Design," Dexter Baker Lectures, Lehigh University, Bethlehem, PA, October 1993.

31. C.J. Coen, Y.C. Chiew, H.W. Blanch, and J.M. Prausnitz, "Salt-Induced Phase Separation of Aqueous Protein Solutions," Annual Meeting of the American Institute of Chemical Engineering, St. Louis, MO, November 1993.
32. Y. Song, S.M. Lambert, and J.M. Prausnitz, "Fluid-Phase Equilibria for Polymer-Solvent Systems from a Perturbed Hard-Sphere Chain Equation of State Based on the Integral Theory of Fluids," Annual Meeting of the American Institute of Chemical Engineering, St. Louis, MO, November 1993.

33. J.M. Prausnitz, "Some New Frontiers in Chemical Engineering Thermodynamics," University of Kyoto, Japan, January 1994.

34. J.M. Prausnitz, "Molecular Thermodynamics for Chemical Process Design," Ethyl Corporation, Baton Rouge, LA, February 1994.

35. D.E. Kuehner, Y.C. Chiew, H.W. Blanch, and J.M. Prausnitz, "A Molecular-Thermodynamic Model for Protein Precipitation in Concentrated Electrolyte Solutions," American Chemical Society Spring Meeting, San Diego, CA, March 1994.

36. H. Zerres and J.M. Prausnitz, "Thermodynamics of Phase Equilibria in Aqueous-Organic Systems with Salt," Spring National Meeting of the American Institute of Chemical Engineering, San Diego, CA, March 1994.

37. J.M. Prausnitz, "Thermodynamics and Separation of Mixtures," Plenary Lecture, Bunsentagung, Berlin, Germany, May 1994.

38. J.M. Prausnitz, "Molecular Thermodynamics for Process and Product Design," Technical University HamburgHarburg, Germany, May 1994

39. J.M. Prausnitz, "Molecular Thermodynamics for Chemical Process and Product Design," University of Manchester, Institute of Science and Technology, United Kingdom, June 1994.

40. A.P. Sassi, "Properties of Hydrogels for Applications in Biotechnology," 4th Research Division, Pharmaceutical Research Division, Eisai, Co., Ltd., Tsukuba, Japan, July 1994, and Department of Chemical Engineering, Kanazawa University, Kanazawa, Japan, August 1994.

41. S.M. Lambert, "Thermodynamics of Polymer Solutions," Dow Chemical Company, Midland, MI, July 1994, and Raychem, Menlo Park, CA, August 1994.

42. C.J. Coen, "Protein Salting-Out Separations: A Fundamental Molecular-Thermodynamic Study," Genencor International, South San Francisco, CA, September 1994. 


\section{Characterization of the Li/Electrolyte Interface*}

Philip N. Ross, Jr., Investigator

\section{INTRODUCTION}

A detailed understanding of the reactions that occur between metallic $\mathrm{Li}$ and the individual molecular constituents of electrolytes used in Li batteries is being developed. Ultrahigh vacuum (UHV) deposition methods are used to prepare ultraclean $\mathrm{Li}$ surfaces of preferred orientation. Molecular films of solvent and/or solute molecules are deposited onto the clean surfaces in UHV at very low temperature. The reaction between $\mathrm{Li}$ and the molecular films is followed using a combination of UHV surface analytical techniques, including Auger electron spectroscopy (AES), secondary ionization mass spectroscopy (SIMS), VUV and $\mathrm{x}$-ray photoelectron spectroscopy (UPES and XPS), and the recently developed variant of angle-resolved PES termed photoelectron diffraction. The connection between films formed on $\mathrm{Li}$ in UHV and films formed at ambient temperature and pressure on $\mathrm{Li}$ in liquid electrolyte is made by the use of a common spectroscopy, ellipsometry. Using the fingerprint method, the ellipsometric signatures obtained in UHV for different surface layers having various known structures and compositions are used to identify the structure and composition of the film formed on the Li electrode in liquid electrolyte. This is a new project initiated late in FY 1993.

\section{Reactivity of Clean Metallic Li Surfaces with Small Molecules: I. Reaction with Dioxygen (Publication 2)}

\section{K. Wang, P.N. Ross, Jr., F.-P. Kong, ${ }^{\dagger}$ and F. McLarnon ${ }^{\dagger}$}

A detailed study was conducted using the combination of ellipsometry and AES of the reaction of clean $\mathrm{Li}$ with $\mathrm{O}_{2}$ by gas dosing in UHV. Thin Li films were deposited on a nickel substrate by the method described in Publication 1 and the film thickness was measured by analysis of the $\mathrm{Li} / \mathrm{Ni}$ AES ratios using elemental sensitivity factors and an exponential attenuation model. Ellipsometric measurements were carried out for several $\mathrm{Li}$ film thicknesses estimated to be from 15 to $150 \AA$ by AES. For this thickness range, it was demonstrated that the ellipsometric parameters $\Delta$ and

\footnotetext{
*This work was supported by the Director, Office of Energy Research, Office of Basic Energy Sciences, Chemical Sciences Division, of the U.S. Department of Energy under Contract No. DE-AC03-76SF00098.
}

$\Psi$ varied (decreased for $\Delta$ and increased for $\Psi$ ) toward the values of bulk $\mathrm{Li}$ as a single-value function of film thickness, being very close to the bulk values at a film thickness of ca. $150 \AA$ ( $\Delta$ and $\Psi$ are defined by $\rho=$ $(\tan \Psi) \mathrm{e}^{\mathrm{i} \Delta}$, where $\rho$ is the ratio of the complex reflection coefficients for $\mathrm{p}$ and $\mathrm{s}$ polarized light components, $\Delta$ and $\tan \Psi$ the relative phase and amplitude change, respectively). This result can be attributed to the facts that the thickness of the films is much smaller than the wavelength of the light ( $4000 \sim 7000 \AA$ ) and that lithium is a very absorbing material so that Drude oscillation does not occur.

The clean films were dosed at low pressure, e.g., $5 \times$ $10^{-8}$ torr, with $\mathrm{O}_{2}$, and uptake of the gases by the $\mathrm{Li}$ was followed simultaneously by spectroscopic ellipsometry and AES. The results showed that $\mathrm{Li}$ is very reactive with $\mathrm{O}_{2}$ at ambient temperature. The oxygen uptake curve measured by AES is shown in Figure 1-1 for different film thicknesses of $\mathrm{Li}$ on $\mathrm{Ni}, 20$ and $150 \AA$. For the thinner film, the entire layer is completely oxidized to a stoichiometry of $\mathrm{L}_{2} \mathrm{O}$ with only 10 Langmuirs ( 1 Langmuir $=10^{-6}$ torr-sec) exposure of dioxygen. For the thicker film, the AES oxygen uptake reached saturation at ca. $20 \mathrm{~L}$ exposure, but because the thickness of the oxide film formed is greater than the escape depth of the oxygen Auger electrons, this saturation does not represent the end of oxidation. This was demonstrated by the ellipsometry data for the $150 \AA$ film shown in Figure 1-2. The $\Delta$ exhibited an initial decrease characteristic of the interaction of dioxygen with other metals, e.g., $\mathrm{Ni}, \mathrm{Cu}, \mathrm{Fe}, \mathrm{Al}, \mathrm{Mg}$, and $\mathrm{Ag}$, then increased sharply approaching the value for the oxygensaturated Ni surface after about $40 \mathrm{~L}$ dosage. The $\Delta-\Psi$ spiral shown in the insert was exactly fitted by an oxidation model of formation of $\mathrm{Li}_{2} \mathrm{O}$ layers that contract due to their higher bulk density than $\mathrm{Li}$, exposing fresh $\mathrm{Li}$ to the dioxygen. This model explains the surprisingly rapid oxidation of the relatively thick (150 $\AA$ ) Li layer. These are important results showing both the power and the limitations of the respective techniques, and the importance of using a combination of spectroscopic methods that complement one another to characterize $\mathrm{Li}$ films in batteries. These films have a complex structure and composition and may vary in thickness from ca. $3 \AA$ to more than a $1000 \AA$.

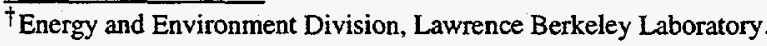

\section{Work in Progress}

Single crystals of both $\mathrm{Al}(111)$ and $\mathrm{Mg}(111)$ will be used as substrates for growing epitaxial thin films of $\mathrm{Li}(111)$ for studies utilizing angle-resolved photoelectron fine structure (ARPEFS). Given satisfactory progress on 


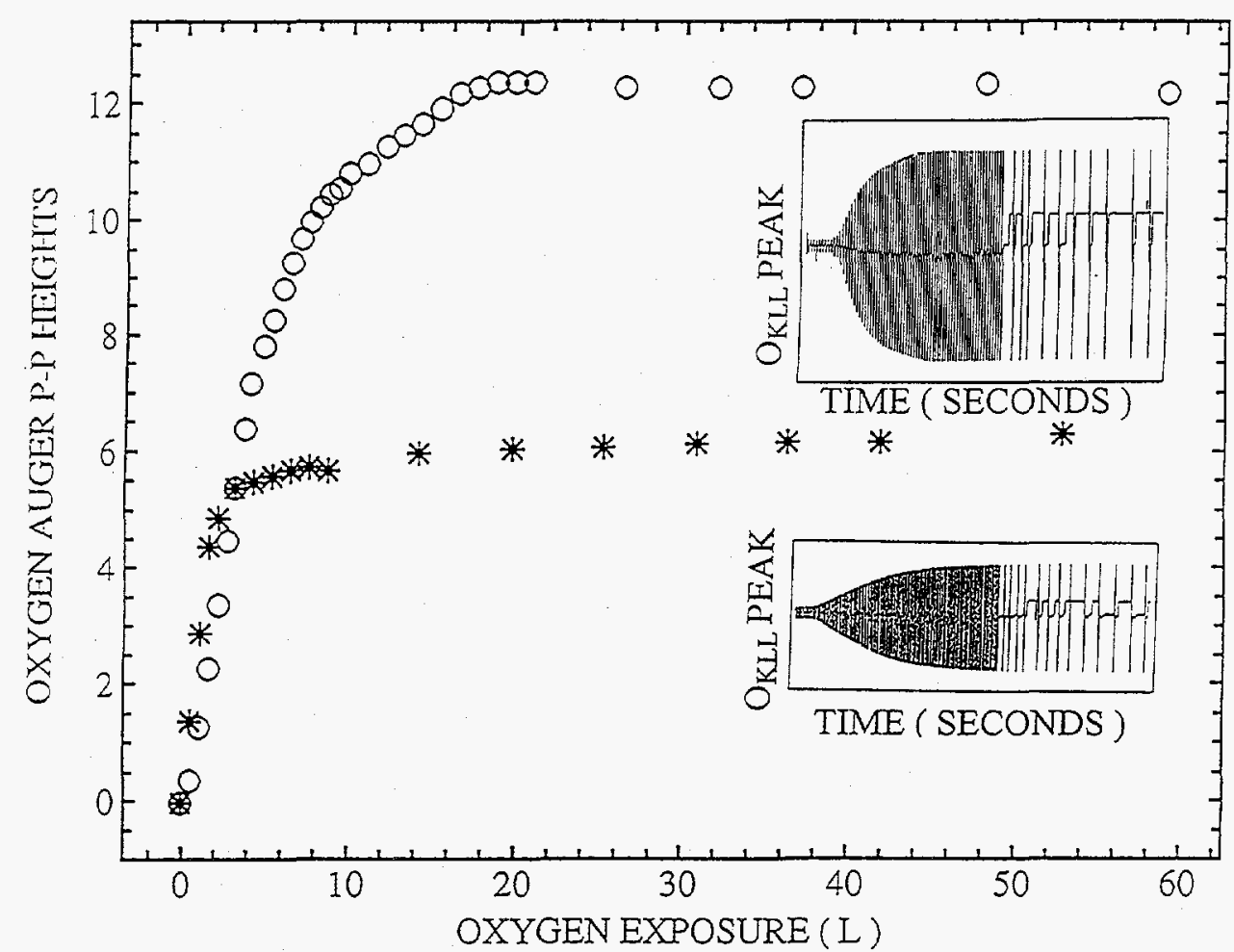

Figure 1-1. (Inserts): Change in $\mathrm{O}$ AES peak at $510 \mathrm{eV}$ in time following exposure of two different Li films to dioxygen gas at $5 \times 10^{-8}$ torr. Resulting $\mathrm{O}$ peak-to-peak heights versus exposure for the two films: (*) $20 \AA$ film; (o) $150 \AA$.

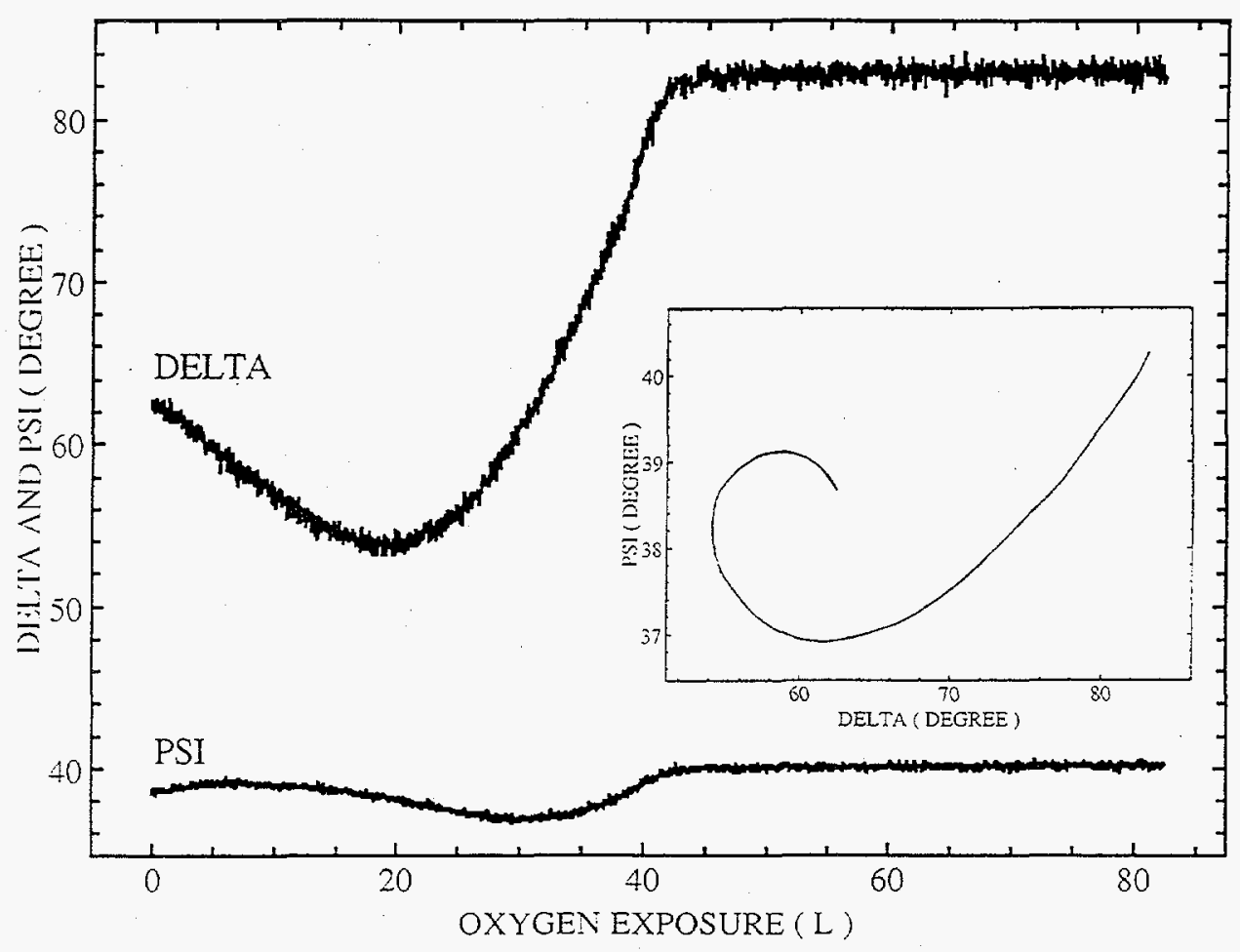

Figure 1-2. Change in the ellipsometric functions $\Delta$ and $\Psi$ with time of exposure to dioxygen as in Figure 1-1 for the $150 \AA$ film. Insert shows the resulting $\Delta-\Psi$ spiral with time as a parameter. 
the growth of clean, epitaxial films of $\mathrm{Li}$, we plan to initiate a photoemission study of the reaction of $\mathrm{Li}$ with $\mathrm{O}_{2}$ to form oxide films in the greater detail afforded by the combination of LEED and photoelectron spectroscopy (especially ARPEFS). This will complement our just completed study of $\mathrm{Li} / \mathrm{O}_{2}$ by ellipsometry. The greater level of detail on the structure and composition of the lithium oxide films as a function of reaction conditions will be used to refine our analysis of the ellipsometric data for the same system. If it turns out that the Li films on $\mathrm{Al}(111)$ and/or $\mathrm{Mg}(111)$ are not sufficiently well ordered for either LEED or ARPEFS, then we will use these substrates to study the effect of alloying $\mathrm{Li}$ with $\mathrm{Al}$ or $\mathrm{Mg}$ on the reactivity of the $\mathrm{Li}$ towards various solvent molecules. However, those experiments will be done at a later time, perhaps as a follow-on to the present study of pure $\mathrm{Li}$. All subsequent photoemission studies would then employ conventional, i.e., not angular resolved, photoemission (XPS and UPS) combined with thermal desorption spectroscopy (TDS). Ni(111) will be used as the substrate because of the convenience of having reversible deposition of $\mathrm{Li}$ on this surface. An atom source, e.g., a source for atomic nitrogen, is being constructed that will enable us to study nitrogen on $\mathrm{Li}$ and to grow $\mathrm{Li}$ nitride films under very well controlled conditions. The growth of $\mathrm{Li}$ nitride films by reaction atomic nitrogen beams would be the next system studied in the ellipsometry chamber.

\section{FY 1994 PUBLICATIONS AND REPORTS}

\section{Other Publications}

1. K. Wang and P. Ross, "Reversible Deposition of Li on Ni(111) in UHV," J. Electrochem. Soc. (in press).

2 K. Wang, P.N. Ross, Jr., F.-P. Kong, and F. McLarnon, "Reactivity of Clean Metallic Li Surfaces with Small Molecules: I. Reaction with Dioxygen," J. Appl. Phys. (submitted). 

LDRD 


\section{Exploration of the Interface Between Homogeneous and Heterogeneous Catalysis: Synthesis and Study of Organometallic Catalysts Supported on Novel Polysiloxane Materials with Controllable Solubility Properties*}

\section{Robert G. Bergman and Bruce M. Novak, Investigators}

\section{INTRODUCTION}

Both homogeneous and heterogeneous catalysis are used by the chemical industry for conversion of feedstocks to commercial chemical products. Heterogeneous catalysts offer the practical advantage of catalyst stability and ease of processing. However, the structures of heterogeneous catalysts and the mechanisms of their reactions cannot be studied easily, and therefore it has historically been difficult to design or modify them in a rational way.

Homogeneous catalysts are more difficult to use, but nevertheless are employed in several large-scale processes (e.g., hydroformylation, methanol carbonylation) because they provide selectivity or chemistry not available in heterogeneous systems. They are almost always more highly characterized than their heterogeneous counterparts, and the mechanisms of their reactions are better understood. As a result, homogeneous catalytic processes are typically more amenable to improvement by systematic structural modification.

In view of the complementary advantages of homogeneous and heterogeneous catalysts, for several years there has been intense interest in developing "hybrid" catalysts that combine the useful properties of both types of systems. An important approach to achieving this goal has been the development of systems in which wellcharacterized homogeneous catalysts are "heterogenized"i.e., they are covalently attached to robust solid supports.

Silica gel is one of the most important and widely-used supports for organometallic catalysts. The goal of this project is the understanding of silica support-catalyst interactions through a continuum approach, wherein the boundary between homogeneous and heterogeneous catalysis systems will be incrementally traversed.

\footnotetext{
*This work was supported by the Laboratory Directed Research and Development funds of the Lawrence Berkeley Laboratory under Department of Energy Contract No. DE-AC03-76SF00098.
}

1. Silica as a Ligand: Reactivity of the IridiumOxygen Bond of $\mathrm{Cp} * \mathrm{Ir}[\mathrm{Silica}](\mathrm{Ph})\left(\mathrm{PMe}_{3}\right)$ (Publication 1)

\section{T.Y. Meyer, K.A. Woerpel, B.M. Novak, and R.G. Bergman}

The novel silica-supported organometallic complex $\mathrm{Cp} * \operatorname{Ir}\left[\right.$ Silica] $(\mathrm{Ph})\left(\mathrm{PMe}_{3}\right)(\mathbf{1})$ was prepared by exchange of silica with the soluble pentamethylcyclopentadienyliridium hydroxide 2 (Figure 1-1). The silica-bound complex 1 was characterized by its reaction with $p$-nitrophenol, regenerating free silica and the iridium $p$-nitrophenoxo complex 4. Chemisorption of the iridium complex was established by the reactions of $\mathrm{Cp} * \mathrm{Ir}\left(\mathrm{OSiMe}_{2}{ }^{\mathrm{t}} \mathrm{Bu}\right)$ $(\mathrm{Ph})\left(\mathrm{PMe}_{3}\right)(6)$ and $\mathrm{Cp} * \operatorname{Ir}\left(p-\mathrm{NHC}_{6} \mathrm{H}_{4} \mathrm{CH}_{3}\right)(\mathrm{Ph})\left(\mathrm{PMe}_{3}\right)(5)$ with silica to produce 1 and an equivalent of $\mathrm{HOSiMe}_{2}{ }^{\mathrm{t}} \mathrm{Bu}$ or $p-\mathrm{NH}_{2} \mathrm{C}_{6} \mathrm{H}_{4} \mathrm{CH}_{3}$, respectively. Treatment of silicabound 1 with the less acidic aryl alcohols phenol, $p$ methoxyphenol, $p$-chlorophenol, and 4-chloro-3,5dimethylphenol resulted in equilibrium mixtures containing the iridium aryloxide products $3,7,8$, and 9 . The apparent equilibrium constants (as defined in Eq. (2)) for these exchange reactions (Eq. (1)), tabulated in Figure 1-1, were found to depend on the acidity of the phenol, with the more acidic ones favoring the formation of aryloxide product. As

$$
\begin{gathered}
{[\mathrm{Ir}]-\text { OSilica }+\mathrm{HOAr} \rightleftharpoons[\mathrm{Ir}]-\mathrm{OAr}+\text { HOSilica }} \\
{[\mathrm{Ir}] \equiv \mathrm{Cp} * \operatorname{Ir}\left(\mathrm{PMe}_{3}\right)(\mathrm{Ph})} \\
\mathrm{K}_{\mathrm{app}}=\frac{[\mathrm{IIr}]-\mathrm{OAr}]\{\text { HOSilica }\}}{\{[\mathrm{Ir}]-\text { OSilica }\} \text { HOAr }]}
\end{gathered}
$$

shown in Figure 1-2, the silica complex 1 reacted with dimethylacetylene dicarboxylate to produce the iridacycle $\mathrm{Cp} * \mathrm{Ir}\left[\mathrm{C}\left(\mathrm{CO}_{2} \mathrm{Me}\right) \mathrm{C}\left(\mathrm{CO}_{2} \mathrm{Me}\right)-o-\mathrm{C}_{6} \mathrm{H}_{4}\right]\left(\mathrm{PMe}_{3}\right)(11)$. Reaction of 1 with ethyl propiolate resulted in formation of $\mathrm{Cp} * \operatorname{Ir}\left(\mathrm{PMe}_{3}\right)(\mathrm{Ph})\left(\mathrm{C} \equiv \mathrm{CCO}_{2} \mathrm{CH}_{2} \mathrm{CH}_{3}\right)(13)$. Complex 1 also activated the C-D bond of benzene- $d_{6}$, replacing the protiated aryl group, to form 1- $d_{5}$ which was characterized by derivatization to $4-d_{5}$. The reactivity of silica-bound 1 was found to be intermediate between that of the monomeric complexes $\mathrm{Cp}$ *Ir(OTf)(Ph)(PMe $)(10)$ and hydroxide 2 , and was believed to be a function of Ir-O bond polarity.

\section{FY 1994 PUBLICATIONS AND REPORTS}

\section{Refereed Journal}

1. T.Y. Meyer, K.A. Woerpel, B.M. Novak, and R.G. Bergman, "Silica as a Ligand: Reactivity of the IridiumOxygen Bond of Cp*Ir[Silica](Ph)(PMe 3$)$," J. Am. Chem. Soc. 116, 10290 (1994); LBL-35850. 

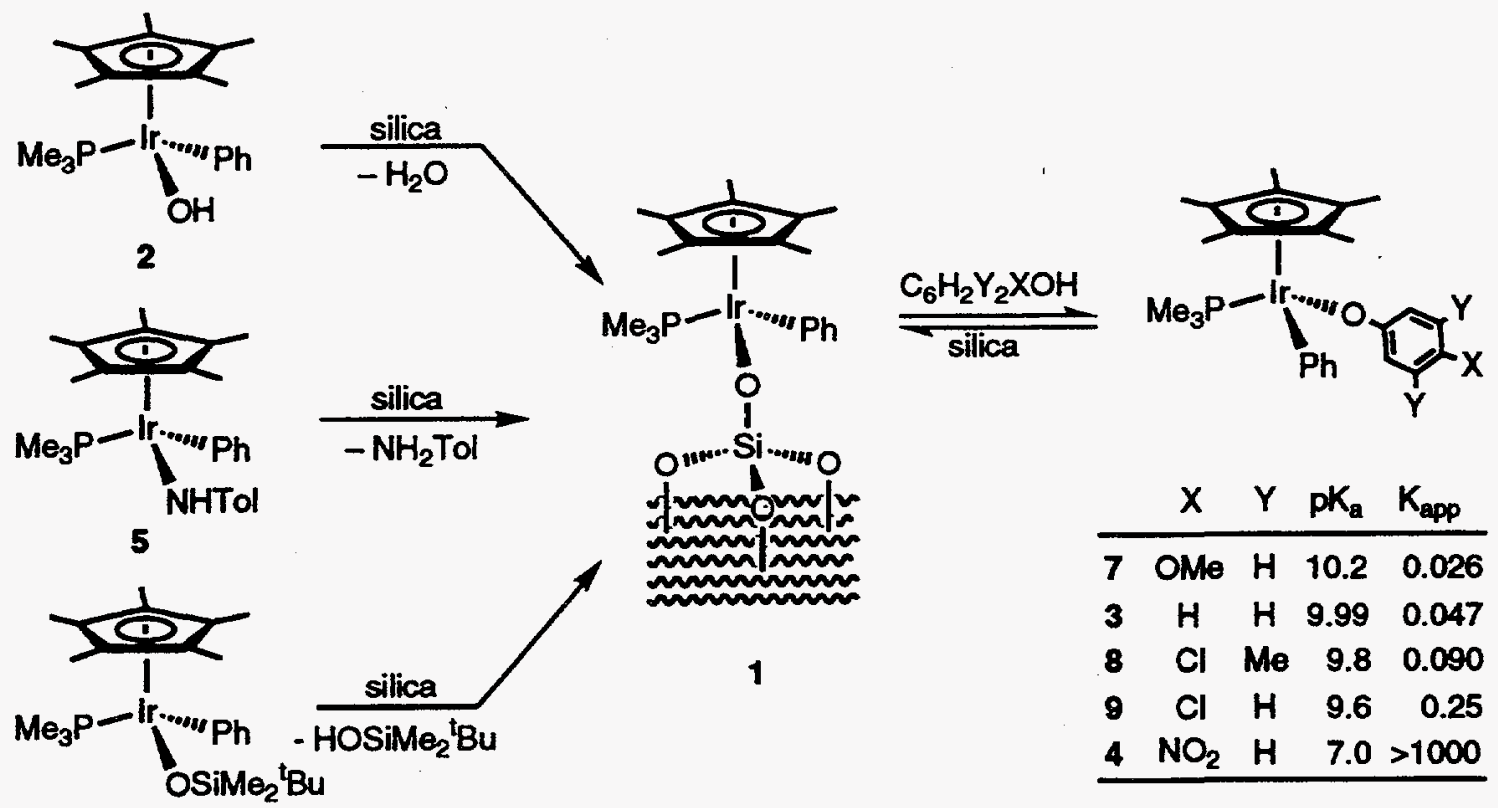

\begin{tabular}{lcccc} 
& X & Y & pKa & K $_{\text {app }}$ \\
\hline 7 & $\mathrm{OMe}$ & $\mathrm{H}$ & 10.2 & 0.026 \\
3 & $\mathrm{H}$ & $\mathrm{H}$ & 9.99 & 0.047 \\
8 & $\mathrm{Cl}$ & $\mathrm{Me}$ & 9.8 & 0.090 \\
9 & $\mathrm{Cl}$ & $\mathrm{H}$ & 9.6 & 0.25 \\
4 & $\mathrm{NO}_{2}$ & $\mathrm{H}$ & 7.0 & $>1000$ \\
\hline
\end{tabular}

6

Figure 1-1. Synthesis of a silica-bound organometallic iridium complex $(1)$ from three different soluble precursors $(2,5$, and 6$)$; apparent $\mathrm{pK}_{\mathrm{a}}$ values for exchange of silica and various substituted phenols at the iridium center.

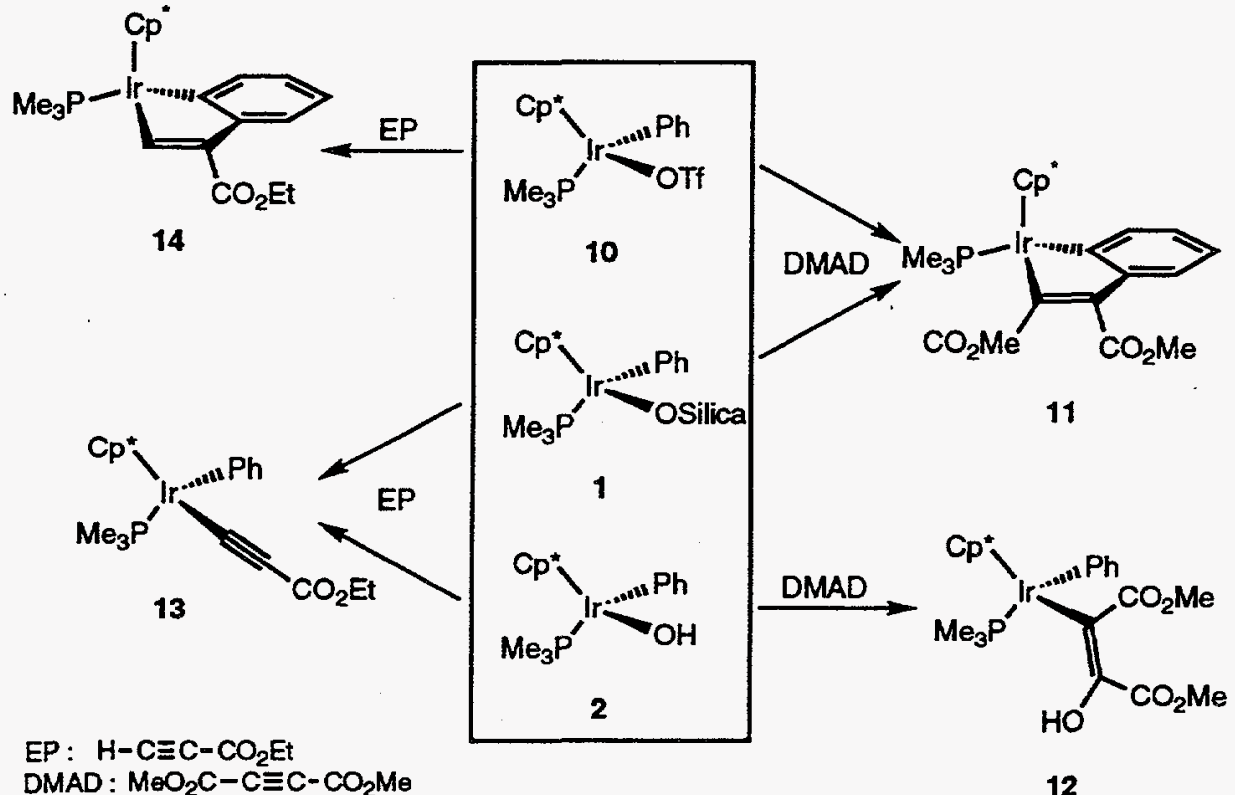

Figure 1.2. Comparison of reactions of silica-bound iridium complex 1 with analogous complexes 2 and 10 . 


\section{Ultrafast Dynamics of Electrons at Surfaces*}

Charles B. Harris, Investigator

\section{INTRODUCTION}

We are developing techniques to examine the spectroscopy and dynamics of surface electronic excitations at interfaces with unprecedented precision. The electronic dynamics of surfaces are involved in many problems of fundamental interest from two-dimensional magnetism to chemisorption. Directly related areas of technological significance include surface photochemistry, laser-surface interactions such as damage mechanisms in optics, surface recombination that can dominate carrier lifetimes at semiconductor interfaces, and magnetic data storage devices.

A complete understanding of excited state dynamics at surfaces requires time, energy, and momentum resolution. Using angle-resolved two-photon photoemission (TPPE), one can study the band structure of electronically excited states at surfaces. We employ an unusual electron time-offlight detection scheme that gives high sensitivity and extreme precision in energy measurements. Liquid helium cooling of the sample to $45 \mathrm{~K}$ enables study of a wide variety of physisorbed and chemisorbed species. Our TPPE apparatus has an order of magnitude better energy resolution than competing techniques and utilizes pico- and femtosecond laser pulses for time-resolved measurements.

\section{Layer-by-Layer Electronic Properties of the} $\mathrm{Xe} / \mathrm{Ag}$ (111) Interface (Publication 2)

\section{C.B. Harris, J.D. McNeill, R.L. Lingle, Jr., R.E. Jordan, and D.F. Padowitz}

Major strides have been made in the study of rare gas films on metal substrates. These experiments show that angle-resolved TPPE can serve as a powerful and general probe of the transition at an interface from two to three dimensions. We have performed layer-resolved twophoton photoelectron spectroscopy of the conduction band of the $\mathrm{Xe} / \mathrm{Ag}(111)$ interface for up to nine atomic layers of

*This work was supported by the Laboratory Directed Research and Development funds of the Lawrence Berkeley Laboratory under Department of Energy Contract No. DE-AC03-76SF00098.

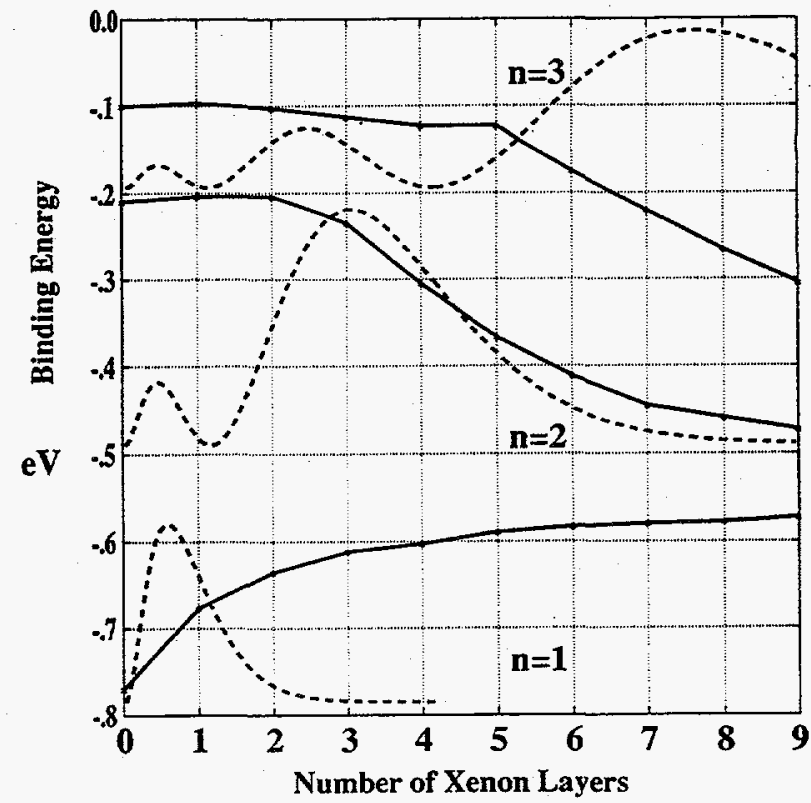

Figure 1-1. The solid lines are the experimental binding energies of an excess electron at the $\mathrm{Xe} / \mathrm{Ag}(111)$ interface for the layer thicknesses indicated. One layer of Xe is approximately $4.1 \AA$ thick. The dashed lines are hydrogenic image potential wave functions for a bare metal surface drawn to scale with layer thickness. This illustrates that a particular image potential state, labeled by its principal quantum number $n$, does not respond to the presence of the adlayer until the layer substantially overlaps with the electron density.

Xe. For multilayers of $\mathrm{Xe} / \mathrm{Ag}(111)$, TPPE shows how the2-D states of the interface evolve toward the 3-D bulk states of crystalline Xe, and the corresponding effective mass measurement by angle-resolved TPPE tracks the spatial extent of the electron as a function of layer thickness. The binding energies of the three lowest quantum states of the conduction band are plotted as a function of layer thickness in Figure 1-1. The $n=1,2$, and 3 bare surface image potential state wave functions are plotted to scale, showing that the energy of a particular state is only perturbed when the layer becomes thick enough to spatially overlap with the electron density distribution of that quantum state.

2. Electrostatics of Metal-Dielectric Interfaces in the Monolayer Limit (Publications 3 and 4)

\section{C.B. Harris, R.L. Lingle, Jr., R.E. Jordan, and J.D. McNeill}

We have recently solved a long-standing puzzle concerning the effect of insulating monolayers on the electrostatic fields near a metal surface. In previous work, ${ }^{1}$ we found that excess electron binding energies on molecular monolayers (including xenon, cyclohexane, and 
n-octane) could be explained without invoking the dielectric response of the monolayer. A systematic study of n-alkanes on $\mathrm{Ag}(111)$ has now revealed that the binding energy of the image potential electron at the monolayer is indeed correlated with both the molecular polarizability and the dielectric constant of the associated bulk material, as shown in Figure 2-1.

A classical electrostatic continuum model indicates that the potential for the metal-insulator interface consists of two principal terms: one is the screened image potential interaction between the electron and the metal while the second is the direct, attractive polarization interaction between the electron and the adlayer. In fact, the attractive interaction between the adlayer and the image electron dominates the screeening effect for monolayers with high dielectric susceptibility. Thus a monolayer may either increase or decrease the binding of an image potential electron to the surface. The two effects were roughly in

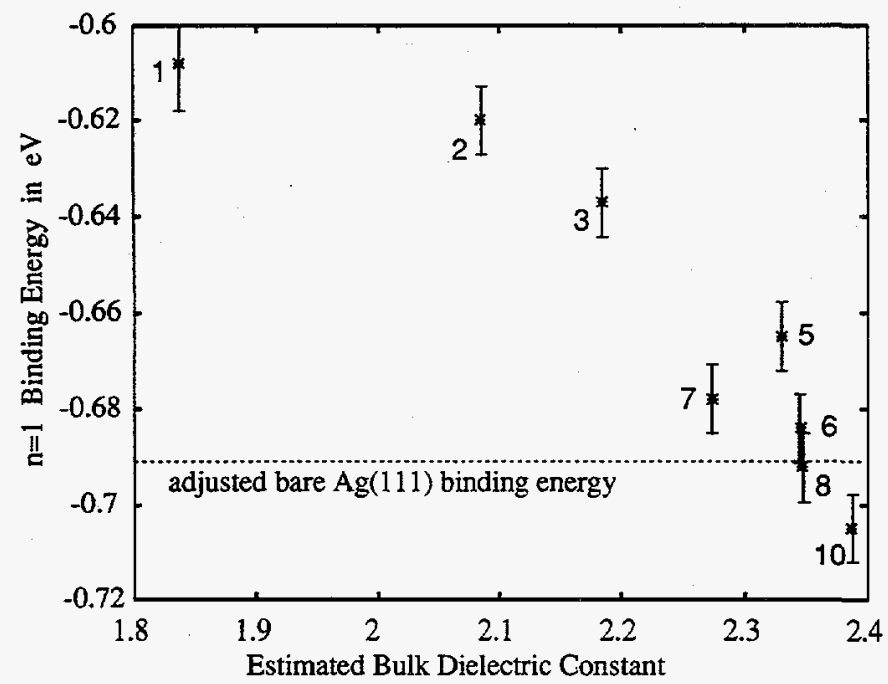

Figure 2-1. Plot of $n=1$ image potential electron binding energy in the presence of monolayers of various $n$-alkanes on $\operatorname{Ag}(111)$. The $x$-axis is the estimated dielectric constant for the associated bulk crystalline material. As the dielectric susceptibility of the layer increases, the binding of the excess electron to the layer dominates screening of the electronmetal interaction, leading to stronger net binding. balance for the previously studied monolayers composed of xenon, cyclohexane, and n-octane.

1. W.R. Merry, R.E. Jordan, D.F. Padowitz, and C.B. Harris, Surf. Sci. 295, 393 (1993).

\section{Electron Localization at Interfaces in 2-D (Publication 1)}

\section{C.W. Harris, R.L. Lingle, Jr., R. E. Jordan, and J.D. McNeill}

We have continued the study of 2-D localization of electrons at metal-alkane interfaces by confirming the predictions of the model we initially applied to this problem in Publication 1. We have observed localized electrons in the bilayer of ethane, n-propane, and n-decane. The ethane case is particularly important, since the theory we applied in Publication 1 predicts that ethane layers would be the shortest alkane to show localization at the interface. We have begun to investigate localization phenomena at interfaces with alkene and aromatic layers.

\section{FY 1994 PUBLICATIONS AND REPORTS}

\section{Refereed Journals}

1. R.L. Lingle, Jr., D.F. Padowitz, R.E. Jordan, J.D. McNeill, and C.B. Harris, "Two-dimensional Localization of Electrons at Interfaces," Phys. Rev. Lett. 72, 2243 (1994).

\section{Other Publications}

2. J.D. McNeill, R.L. Lingle, Jr., R.E. Jordan, D.F. Padowitz, and C.B. Harris, "Layer-by-layer Development of the Electronic Structure of the Xe/Ag(111) Interface," Phys Rev. Lett. (in preparation).

3. R.L. Lingle, Jr, R.E. Jordan, J.D. McNeill, and C.B. Harris, "Dielectric Effects at Monolayer Insulator-on-Metal Interfaces," Surf. Sci. (submitted).

4. R.E. Jordan, R.L. Lingle, Jr., J.D. McNeill, D.F. Padowitz, and C.B. Harris, "Electrons at Metal-Insulator Interfaces II: Adsorbate Morphology and Electronic Structure on Alkane Multilayers," Surf. Sci. (in preparation). 


\section{Structure and Chemistry of Non- metallic Adsorbates at Semiconductor Interfaces Investigated by Synchrotron Radiation Techniques*}

\author{
David K. Shuh, Investigator
}

\section{INTRODUCTION}

This research project utilizes two complementary Synchrotron Radiation (SR) surface characterization methodologies to determine the structural and chemical properties of binary semiconductor material interfaces with simple adsorbates. The results of the SR investigations will provide both fundamental and applied information about the nature of chemisorption on compound semiconductors that are needed to improve existing semiconductor materials processing technologies. Beamlines capable of supporting the SR techniques employed for this work will become available in the near term at the Advanced Light Source (ALS). A principal consideration is development of a scientific program and experimental equipment that will take full advantage of the unique ALS facility resources.

The research employs several SR techniques. X-ray Standing Waves (XSW), Soft X-ray Photoelectron Spectroscopy (SXPS), and Near Edge X-ray Absorption Fine Structure (NEXAFS) are used to characterize the adsorbate-semiconductor interfaces in a direct, surface sensitive manner. XSW is utilized for the determination of the structural parameters, SXPS to characterize the chemical behavior of the adsorbate-semiconductor systems, and NEXAFS for electronic information. These photon-in electron spectroscopy experiments primarily use dedicated user-accessible endstations at the electron storage rings of the Stanford Synchrotron Radiation Laboratory (SSRL) and the National Synchrotron Light Source (NSLS), although there have been initial activities at the ALS. In conjunction with the SR experimental program and efforts, a simple surface science system with limited analytical capabilities continues to be developed to support the SR projects. This modest surface science capability allows for preliminary research on experimental systems of interest, testing of experimental apparatus, and has been upgraded to serve as a primitive SR endstation.

*This work was supported by the Laboratory Directed Research and Development funds of the Lawrence Berkeley Labortory under Department of Energy Contract No. DE-AC03-76SF00098.
1. XSW Results from the $\mathrm{J} / \mathrm{GaAs}(110)$ Interface

\section{W.M. Tong and D.K. Shuh}

The I/GaAs(110) interface geometry has been elucidated for the first time utilizing the XSW technique. The chemisorption of halogens on the GaAs(110) chemically etch the semiconductor material and forms surface reaction products with high oxidation states, but the iodine $\operatorname{GaAs}(110)$ system is unique in that it does neither at room temperature. The structure of the $\mathrm{I} / \mathrm{GaAs}(110)$ interface may form similarly to an ideal epitaxially continued layer structure bulk termination or an epitaxial on top structure, both of which may exhibit passivating properties. The XSW measurements have been performed at SSRL Beamline 3-3 (Jumbo). The bulk and iodine back reflection XSW spectra shown in Figures 1-1 and 1-2 were collected in the constant initial state mode from

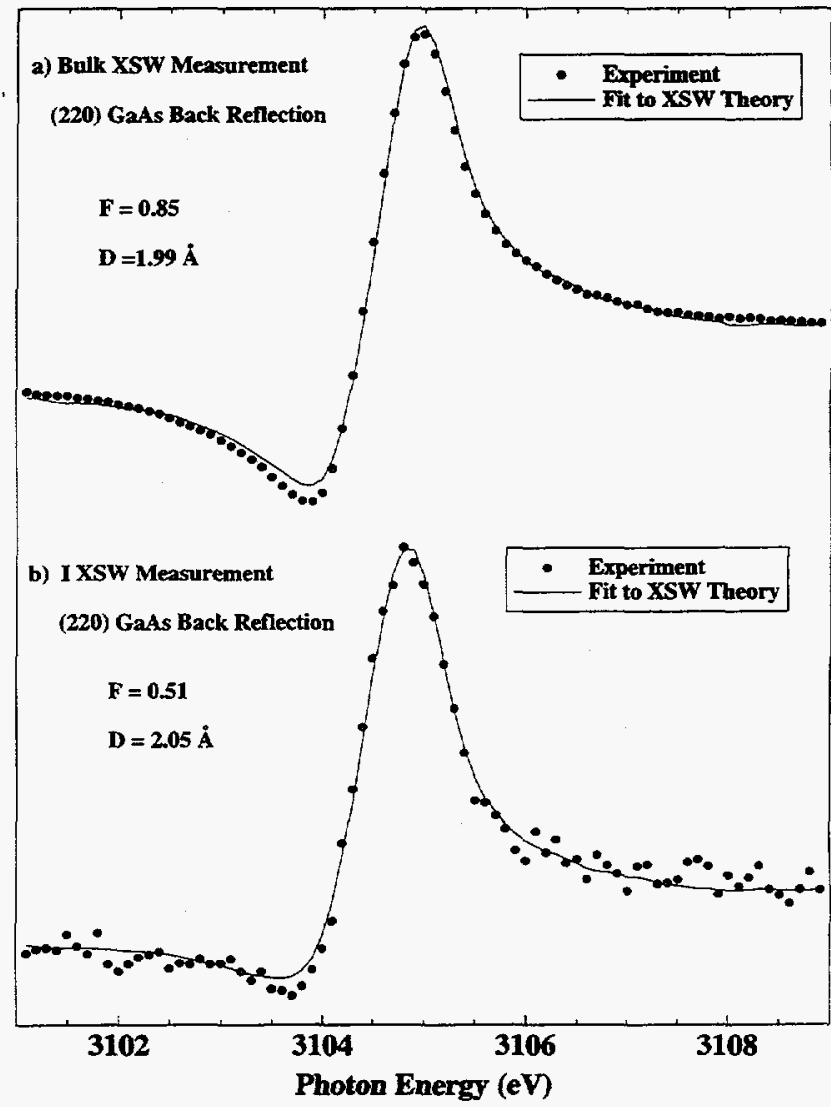

Figure 1-1. The (a) bulk and (b) I XSW profile measurements from the (220) back reflection of the $\mathrm{L} / \mathrm{GaAs}(110)$ interface. The experimental points are represented by the filled circles. The least squares fits to the experimental data, simulated by Egn. (1) and from which the structural parameters have been extracted, are shown by the solid lines. (XBD 9501 00160) 


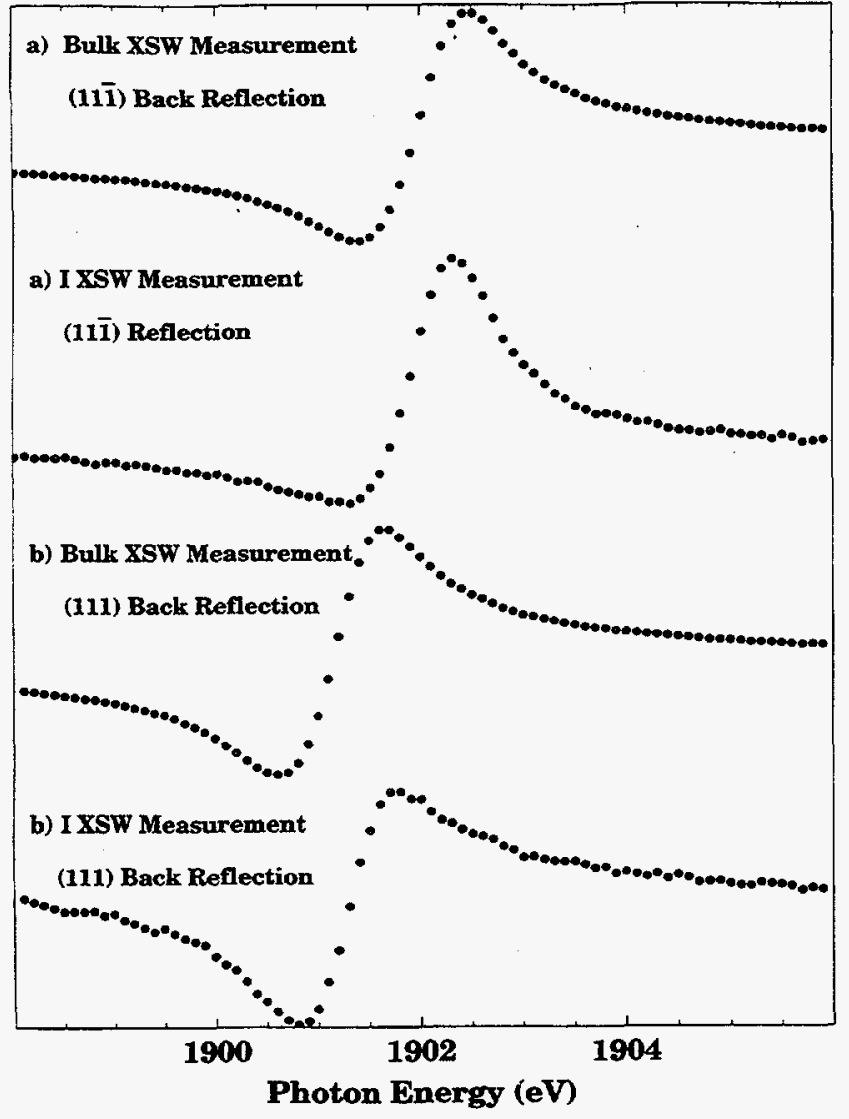

Figure 1-2. The bulk and I XSW yields from the measurements of the (a) (11i) and the (b) (111) back reflections from the $1 / \mathrm{GaAs}(110)$ interface, respectively. The experimental data are shown as filled circles. (XBD 9501-00161)

photoemission or from the total photocurrent, respectively. The iodine XSW signals were obtained from the difference between photoelectron signals collected at the I $3 \mathrm{p} 3 / 2$ peak and those from background. The interface structural information is then derived from a two parameter computer simulation ( $\mathbf{F}$ and $\mathbf{D})$ of

$$
\text { XSW yield }=1+R(E)+2 F \sqrt{R} \cos (v(E)-2 \pi D)
$$

in which $R$ is the reflectivity, $v$ is the reflectivity phase, $F$ is the coherent fraction, and $\mathbf{D}$ is the coherent distance to the Bragg plane of interest. Thus, the amplitude of an XSW spectrum is related to the coherent fraction $(F)$ and the energy position to the phase (D). The reflectivity (not shown) combined with the bulk XSW establishes the experimental sensitivity and resolution, whereas with the I XSW yields the structural parameters of the adsorption geometry.

Figure 1-1 shows the results of the (220) XSW measurements for both the bulk crystal and the I saturated surface. The bulk coherent fraction is low (0.85) as a result of the beamline optical characteristics but the planar spacing is exact. The I XSW has a coherent fraction of 0.51 , indicating some disorder of the I overlayer, but gives an iodine-GaAs distance of $2.05 \AA$ that is nearly the same as the bulk GaAs (220) spacing of $1.99 \AA$. This is the first successful (220) $\mathrm{GaAs}$ reflection experiment that has been obtained on the Jumbo beamline. The $\{111\} \mathrm{XSW}$ experimental reflections are presented in Figure 1-2 and have been fit on a trial basis. The coherent fractions are similar to the (220) reflection and yield interface distances that are characteristic of a bulk termination model. This supports the notion that iodine may form an ideal and passivating interface with $\mathrm{GaAs}(110)$.

\section{Surface Science System/Basic SR Endstation and ALS Beamline 8.0}

\section{W. M. Tong and D.K. Shuh}

The modest laboratory surface science system continues to be employed to perform simple measurements and tests of experimental apparatus. The initial efforts to enhance the capabilities of the system permitting its use as a most basic SR endstation have been implemented. Improvement in the analytical capabilities and instrumentation of the system have also been achieved to meet increased experimental characterization requirements. These ongoing laboratory efforts are essential to complement the SR program, enhance the productivity of the outside userbased SR efforts, and for the prerequisite investigations of experimental systems that are required for successful experimentation at SR sources.

The SXPS investigations will be additionally facilitated by recent membership in the Beamline 8.0 Participating Research Team at the ALS that will provide dedicated beamtime. Valuable contributions to the commissioning of the beamline and beamline endstation have been made.

\section{FY 1994 PUBLICATIONS AND REPORTS}

\section{Refereed Journal}

1. J.J. Jia, T.A. Callcott, J. Yurkas, A.W. Ellis, F.J. Himpsel, M.G. Samant, J. Stohr, D.Ederer, J.A. Carlisle, E.A. Hudson, L.J. Terminello, D.K. Shuh, and R.C.C. Perera, "First Experimental Results From the IBM/TENN/ TULANE/LBL Undulator Beamline at the ALS," Rev. Sci. Instrum. 66, 1442 (1995); LBL-36716.

\section{Other Publications}

2. W.M. Tong, D. K. Shuh, T. Kendelewicz, J. C. Woicik, and P. Pianetta, "Structure of Iodine on GaAs(110) by X-ray Standing Wave Analysis," (in preparation). 
Work for Others 


\section{UNITED STATES OFFICE OF NAVAL RESEARCH}

\section{Superconducting Properties of High- $\mathbf{T}_{\mathbf{c}}$ Oxides*}

\author{
Vladimir Z. Kresin, Investigator
}

\section{INTRODUCTION}

High- $T_{c}$ oxides are layered doped systems; their properties, and the energy spectrum, in particular, strongly depend on the oxygen stoichiometry, level of the doping, etc. Charge transfer is greatly affected by the oxygen content.

Oxygen depletion, as well as overdoping, lead to an appearance of localized magnetic moments and, consequently, to a peculiar gapless superconducting state. The gaplessness has a great impact on the spectroscopy of the cuprates. The behavior of the critical magnetic field is also affected by the presence of magnetic impurities and their ordering at low temperatures.

John-Teller instability also affects charge transfer. This non-adiabaticity leads to isotopic dependence of the carrier concentration, $T_{c}$ and the penetration depth.

A new type of tunneling spectroscopy ("break" junction) allows one to obtain important information. In addition, the "break" Josephson junctions are promising from a point of view of various applications.

\section{Energy Spectrum of Layered Systems: Gaplessness} and the Implications (Publications 2, 5, 7, and 9)

\section{V.Z. Kresin and S.A. Wolf $f^{\dagger}$}

The presence of different low-dimensional structural units along with a short coherence length leads to the formation of a two-gap spectrum, that is, to a double-peak structure in the superconducting density of states (e.g., Y$\mathrm{Ba}-\mathrm{Cu}-\mathrm{O}$ ). The presence of magnetic impurities leads to a gapless state. As opposed to the usual one-gap isotropic model, the region of gaplessness is extended, so that one can observe the effect of gaplessness without a noticeable shift in $T_{c}$. Strong anisotropy of the pairing interaction within the planes of layered superconductor also leads to a similar extension of the region of gapless behavior in the presence of magnetic impurities. This gaplessness has a

*This work was supported by the U.S. Office of Naval Research under Contract No. N00014-92-F0002 through an agreement with the U.S. Department of Energy under Contract No. DE-AC03-76SF00098. great impact on the spectroscopy of the cuprates, particularly for nonstoichiometric compounds.

The concept of gaplessness provides a unified and consistent description of experimental data related to the symmetry.

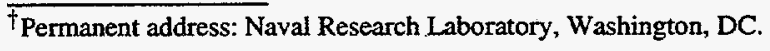

2. Nonadiabaticity: Isotopic Dependent of the Carrier Concentration, $T_{c}$ and the Penetration Depth (Publications 1, 4, and 8)

\section{V.Z. Kresin and S.A. Wolft}

Nonadiabaticity leads to a peculiar dependence of the carrier concentration (and hence $T_{c}$ ) on the isotope substitution. This specific isotope effect, manifested in the doped superconductors, is characterized by the minimum value of the isotope coefficient at $T_{c}=T_{c \text {,max }}$. The value of the carrier concentration affects not only $T_{c}$, but a number of other parameters as well. Among them, the penetration depth, pressure coefficient, etc. As a result, one can predict the isotopic dependence of the penetration depth.

${ }^{\dagger}$ Permanent address: Naval Research Laboratory, Washington, DC.

\section{Collective Excitations in Cluster-Type}

Compounds: Application to Fullerides and QuantumDot Arrays (Publication 3)

\section{V.Z. Kresin and V.V. Kresin ${ }^{\dagger}$}

We analyze collective excitations of carriers in threeand two-dimensional crystals made up of cluster-type units (fullerenes, quantum dots, etc.). This class of compounds is unique in that the electrons are essentially confined to the individual clusters, while the latter are both large in size and close packed. We derive a general expression for the dispersion law of conduction-band plasmons, and apply it to alkali-metal-doped fullerides and quantum-dot arrays. We find that the dispersion relation is modified from the case of a homogeneous electron gas: it becomes measurably steeper and acquires a downward slope. This change is due to the appearance of a term that is determined by the density distribution of electrons within a unit cluster.

\footnotetext{
†ermanent address: University of Southern Califomia, Los Angeles.
} 
4. Single-Particle Tunneling and Josephson Effect in Single-Crystal Break Junctions (Publication 6)

Ya.G. Ponomarev, ${ }^{\dagger}$ B.A. Aminov, M.A. Hein, ${ }^{\dagger}$ H. Heinrichs, ${ }^{\ddagger}$ V.Z. Kresin, G. Muller, ${ }^{\ddagger}$ H. Piel, $\ddagger$ K. Rosner, ${ }^{\S}$ S.V. Tchesnokov, ${ }^{\dagger}$ E.B. Tsokur, ${ }^{\dagger} D$. Wehler, ${ }^{\ddagger}$ K. Winzer, ${ }^{\S}$ A.V. Yarygin, ${ }^{\dagger}$ and K.T. Yusupov ${ }^{\dagger}$

The current-voltage characteristics of break junction in single crystals of $\mathrm{YBa}_{2} \mathrm{Cu}_{3} \mathrm{O}_{7-\mathrm{x}}$ and $\mathrm{YbBa}_{2} \mathrm{Cu}_{3} \mathrm{O}_{7-\mathrm{x}}$ at helium temperature have been studied. For a $\mathrm{YBa}_{2} \mathrm{Cu}_{3} \mathrm{O}_{7-\mathrm{x}}$ Josephson junction, Fiske resonances in low magnetic fields have been observed in the $\mathrm{I}(\mathrm{V})$ characteristic, thus proving the tunneling nature of the contact. The reduced velocity of the electromagnetic wave in the contact area has been calculated to be $c / c_{0}=0.018$ ( $c$ is the Swihart velocity). The ratio of the barrier thickness $d_{\mathrm{I}}$ to the relative dielectric constant of the barrier $\varepsilon$ was found to be $d_{\mathrm{I}} / \varepsilon=0.09 \mathrm{~nm}$. In the quasiparticle tunneling regime, the $\mathrm{YbBa}_{2} \mathrm{Cu}_{3} \mathrm{O}_{7-\mathrm{x}}$ break junctions showed in some cases $\mathrm{I}(\mathrm{V})$ characteristics with a relatively small excess current at "sub-gap" voltages and a steeper rise of the current at the "gap" voltage $V_{g}=2 \Delta / e$. The "BCS-like" form of the observed I(V) characteristics seems to be in favor of s-wave superconductivity.

\footnotetext{
† Permanent address: Moscow State University, Moscow, Russia.

‡Permanent address: Universitat Wuppertal, Wuppertal, Germany

$\S_{\text {Permanent address: Universitat Gottingen, Gottingen, Germany. }}$
}

\section{Localized Magnetic Moments in Layered Systems (Publication 9)}

\section{Yu.N. Ovchinnikov ${ }^{\dagger}$ and V.Z. Kresin}

The presence of magnetic impurities in layered system and the formation of a "spin glass" at low temperature drastically affect the spin-flip scattering, and this is manifested in spectroscopy and transport properties. Moreover, the nature of the dependence $\mathrm{H}_{\mathrm{c} 2}(\mathrm{~T})$ is greatly affected by the presence of magnetic impurities and their ordering at low temperatures. The scattering leads to a change in the sign of the curvature, and consequently to an increase in the value $H_{c 2}$. The theory allows one to explain recent experimental data with the overdoped cuprates that exhibits behavior that is drastically different from the conventional picture.

\footnotetext{
†Permanent address: Landau Institute for Theoretical Physics, Moscow, Russia.
}

\section{FY 1994 PUBLICATIONS AND REPORTS}

\section{Refereed Journals}

1. V.Z. Kresin and S.A. Wolf, "Microscopic Model for the Isotope Effect in the High- $T_{c}$ Oxides," Phys. Rev. B49, 3652 (1994).

2. S.A. Wolf and V.Z. Kresin, "Symmetry of the Pairing, and Mechanism of Superconductivity in the Cuprates," Phys. C235-240, 2413 (1994).

3. V.V. Kresin and V.Z. Kresin, "Conduction-Band Plasmons in Cluster-Type Compounds: Application to Fullerides and Quantum-Dot Arrays, Phys. Rev. B49, 2715 (1994).

4. V.Z. Kresin and S.A. Wolf, "Symmetry, Mechanism of the Pairing, and the Isotope Effect in the Cuprates," J. Superconductivity 7, 531 (1994).

5. V.Z. Kresin and S.A. Wolf, "Symmetry of the Pairing State in the High-Tc Oxides," J. Superconductivity 7, 865 (1994).

\section{Other Publications}

6. Ya.G. Ponomarev, B.A. Aminov, M.A. Hein, H. Heinrichs, V.Z. Kresin, G. Muller, H. Piel, K. Rosner, S.V. Tchesnokov, E.B. Tsokur, D. Wehler, K. Winzer, A.V. Yarygin, K.T. Yusupov, "Josephson Effect and SingleParticle Tunneling in $\mathrm{TBa}_{2} \mathrm{Cu}_{3} \mathrm{O}_{7-\mathrm{x}}$ and $\mathrm{YbBa}_{2} \mathrm{Cu}_{3} \mathrm{O}_{7-\mathrm{x}}$ Single-Crystal Break Junctions," Phys. (in press).

7. V.Z. Kresin and S.A. Wolf, "Gaplessness and Properties of Layered Superconductors: Application to $\mathrm{High}-\mathrm{T}_{\mathrm{C}}$ Cuprates," Phys. Rev. B (in press).

8. V.Z. Kresin and S.A. Wolf, "Non-Adiabaticity and Novel Isotope Effect in the Doped Cuprates," in Proceedings of the International Conference on Lattice Dynamics (in press).

9. Yu.N. Ovchinnikov and V.Z. Kresin, "Critical Magnetic Field in Layered Superconductors," Phys. Rev. B (in press).

\section{Invited Talks}

10. V.Z. Kresin, "Energy Spectrum in the High $T_{c}$ Oxides," Center of Superconductivity, University of Houston, Houston, TX, October 1993.

11. V.Z. Kresin, "High $T_{\mathfrak{c}}$ Superconductivity," A\&M University, College Station, TX, October 1995.

12. V.Z. Kresin, "Gapless Superconducting State," Center of Superconductivity, University of Maryland, College Park, MD, March 1994.

13. V.Z. Kresin, "Fundamentals of Superconductivity," Lectures as Guest Professor, University of Wuppertal, Wuppertal, Germany, June 1994.

14. V.Z. Kresin, "Superconducting State in Nature (e.g., superconductivity and astrophysics; complex molecules; nuclear matter)," University of Wuppertal, Wuppertal, Germany, June 1994. 
15. V.Z. Kresin, "Nature of High $T_{c}$ Superconductivity," Forschungszentrum, Karlsruhe, Germany, June, 1994.

16 V.Z. Kresin, "Spectroscopy and $T_{c}$ of High $T_{c}$ Oxides," Institute fur Festkorperforschung, Julich, Germany, June 1994.

17. V.Z. Kresin, "Properties of High $T_{c}$ Cuprates," University of Groningen, Groningen, Netherlands, June 1994.
18. V.Z. Kresin, "Non-Adiabaticity and High $\mathbf{T}_{c}$," International Conference on Lattice Dynamics, Bled, Slovenia, September 1994.

19. V.Z. Kresin, "Theory of High $T_{c}$," Center of Superconductivity, Cavendish Laboratory, University of Cambridge, England, September 1994.

20. V.Z. Kresin, "Spectroscopy of High $T_{c}$ Oxides," University of Birmingham, England, September 1994. 


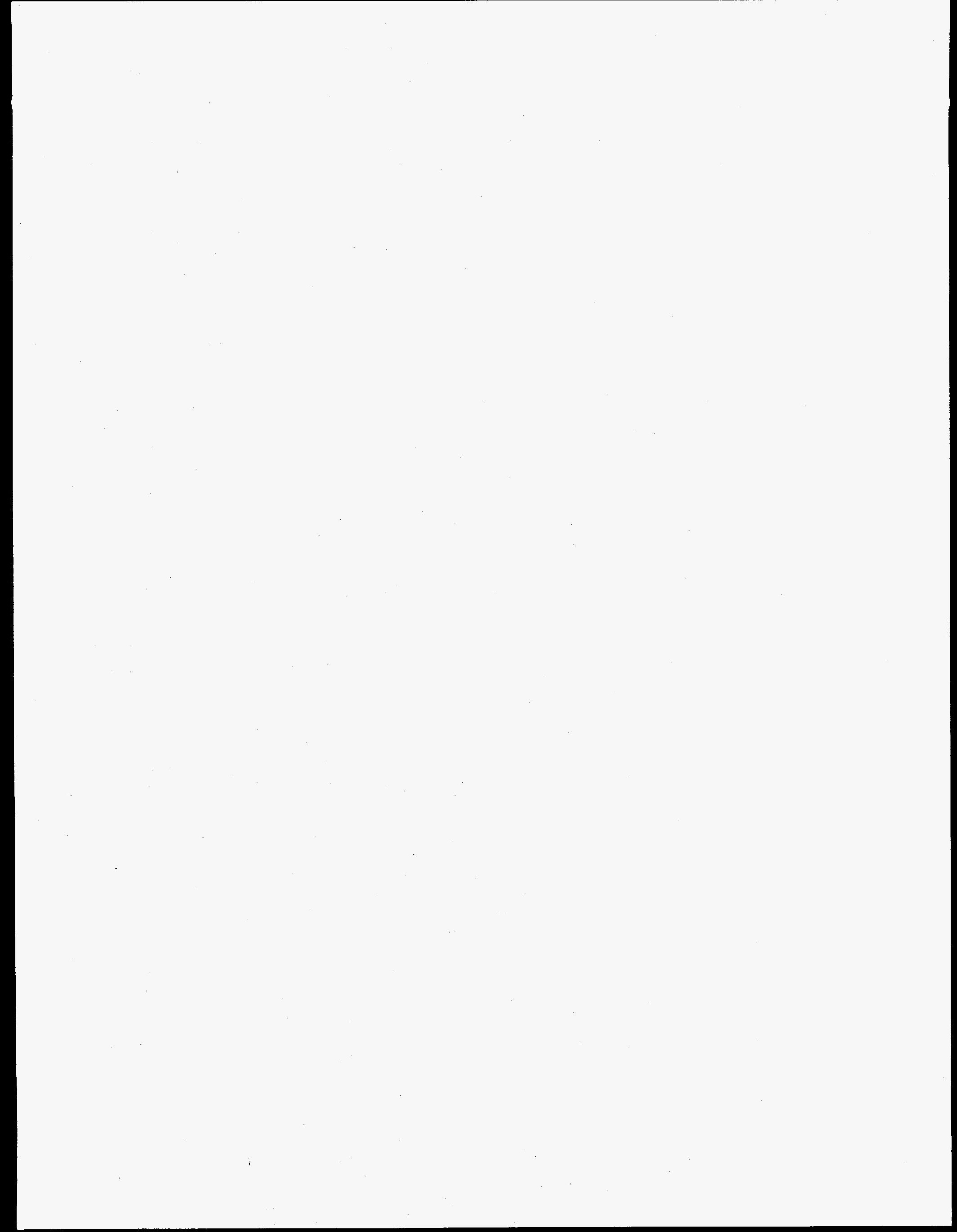


Appendices 


\section{APPENDIX A}

\section{DIVISION PERSONNEL}

\section{Scientific Staff}

Investigators

Scientists, Postdoctoral

Researchers, and Other Research Staff

$\begin{array}{lll}\text { Richard A. Andersen } & \text { L.L. Blosch } & \text { W.W. Lukens, Jr. } \\ & \text { M.A. Petrie } & \text { M.D. Smith }\end{array}$

Neil Bartlett

P. Botkovitz

J.C. Allman

G.M. Lucier

S.H. Elder

B.-C. Shen

L.C. Chacon

J. Munzenberg

Alexis T. Bell

J.N. Carstens

D.B. Clarke

C.S. Gittleman

M.J. Sandoval

I.A. Fisher

S.C. Su

S.F. Brionne

J. Fung

D.M. Kato

T. Komaya

A.K. Murali

Robert G. Bergman

Yongqin Chen

Eugene D. Commins

Robert E. Connick

Norman M. Edelstein
J.J. Bucher

K.M. Murdock

K.P. Osborne

A. Zalkin

\begin{abstract}
A.A. Bengali
T.Y. Meyer
\end{abstract}

M.A. Aubart

A.M. Baranger

M.D. Butts

D.A. Dobbs

T.A. Hanna

P.L. Holland

M.P. Blackwell

B.C. Regan

S.B. Ross

\section{Y.-X. Zhang}

A.-D.L. Nguyen

B.A. Arndtsen M.J. Tauber

R.C. Gatti

S. Hubert

E.A. Hudson

N. Kaltsoyannis
A.R. Smith
J. Sytsma
D.H. Templeton
L.K Templeton
L.J. Terminello 
Harvey A. Gould

Zahid Hussain

Charles B. Harris

T. Lian M.C. Asplund

R.L. Lingle

A. Belkacem

T.P. Dinneen

K. Momberger

D.W. Spooner

S.E. Gadd

N.-H. Ge

J.K. Hane

W.R.A. Huff

S.A. Kellar

E.J. Moler

B.J. Petersen
A.H. Sorensen

K.M. Street

R.E. Jordan

J.C. King

J.D. McNeill

S.E. Bromberg

C.T. Vala
Y. Chen

E.A. Hudson

T. Reich

H. Wu

Y. Zheng

$X$. Zhou
Harold S. Johnston

Vladimir Z. Kresin

Andrew H. Kung

Yuan T. Lee

William A. Lester, Jr.

Richard Marrus

William H. Miller

C. Bradley Moore

Daniel M. Neumark
R.N. Barnett

C.W. Greeff

S.K. Kim

C.-K.K. Ni

J. Xie

$\mathrm{X}$. Yang

A.M. Solvia

A.G. Suits

Berrie

D.A. Blank

D.W. Boo

A.S. Bracker

J.D. Chesko

W.R. Brown

B.B. Birkett

S.M. Auerbach

J.D. Gezelter

R.A. Alvarez

H.P. Beal

C.C. Arnold

D.W. Arnold
H. Hou

C.A. Longfellow

T.-T. Miau

S.W. North

L.A. Smoliar

M.M. Soto

S. Keshavamurthy

L.W. Poirier

E.A. Wade

D.R. Cyr

E.M. Ross
C.-Y.Ng

C.-L.C. Cheng

C.-W. Hsu

R.-I. Kaiser

G.-Y. Liu

K.-T. Lu

D. Stranges

M.J.J. Vrakking

J.A. Odutola

Z. Sun
R. Hernandez

W.H. Thompson 
Scientists, Postdoctoral

Investigators Researchers, and Other

Graduate Students

Guests, Affiliates

Research Staff

Kenneth S. Pitzer

John M. Prausnitz

Michael H. Prior

Kenneth N. Raymond

J. Jin

H.A. Gasteiger

K. Wang

W.M. Tong

B.M. Boyd

A.P. Sassi

J. Wu

A.L. Schiozer

S. Jiang

C.S. Oakes
S.J. Franklin
V.L. Freeman

C. Sunderland

D.W. Whisenhunt, Jr.

S.C. Bardin

J.-P.L. Briand

J.R. Crespo Lopez-Urrutia

L. de Crespin de Billy

R.R. Haar

H. Khemliche

M. Nectoux

K. Langemann

B. Lulay-Bryan

C.O. Roth

S.U. Stutz

P.G. Allen

E.R. Writer

C.G. Lugmair
D.S. Brown
B.A. Siesel
J.K. Cammack
A.H. Singh
S. Kumaraswamy
B. Bodenmann
J.-T. Hwang
D.W. Tien 


\section{SUPPORT STAFF}

Division Administrative Staff

Division Administrator: Linda R. Maio

Administration

L.M. Aubert

T.J. Lynem

A. Winters

Travel

S. Quarello*

Administrative/Secretarial Staff

C. Clark

I. Coble

J. Denney

M. Forman

C. Fowler

C. Gliebe

J.M. Gotay

I. Katsumoto
Personnel/Financial

T.J. Lynem

A. Winters

Technical Support

M.A. Graham
Purchasing

L.M. Aubert

T.R. Lynem

A. Winters

R. Rivas

E.A. Lawhead

L. Ross

H. Manning

S. Montgomery

F.J. Semwogerere

V. Narasimhan

M.F. Noyd

J. Powers

T.C. Peoples

J.G. Rivas

P. Southard

K. Steele

P. Takahashi

D. Williams

A. Wingfield

*Materials Sciences. 


\section{APPENDIX B}

\section{INDEX OF INVESTIGATORS*}

Andersen, Richard A. 62

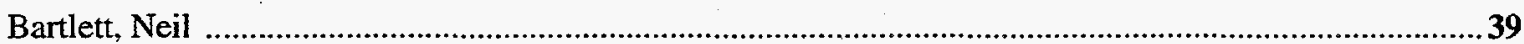

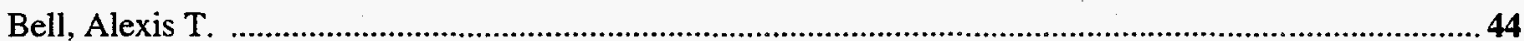

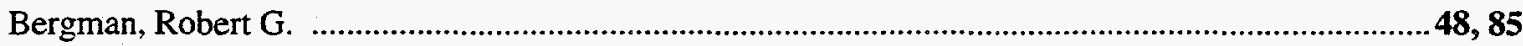

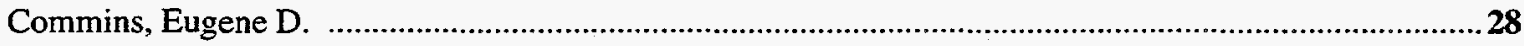

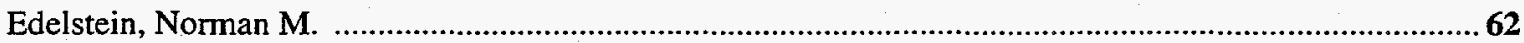

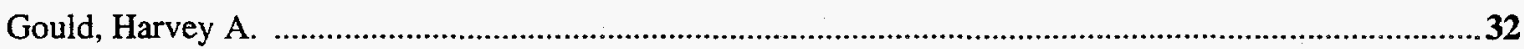

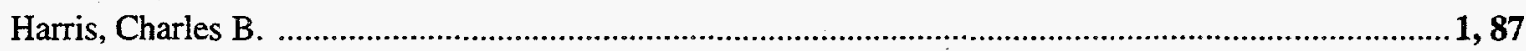

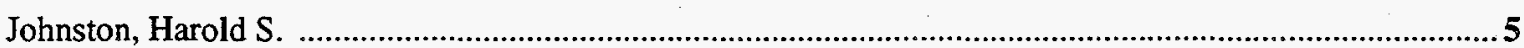

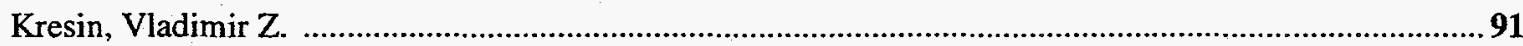

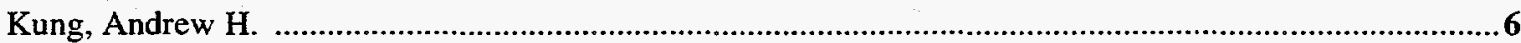

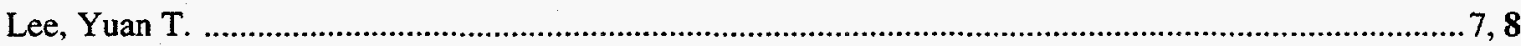

Lester, Jr., William A. ............................................................................................................................ 12

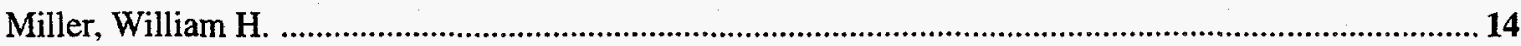

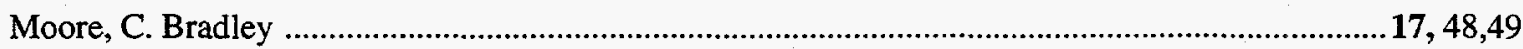

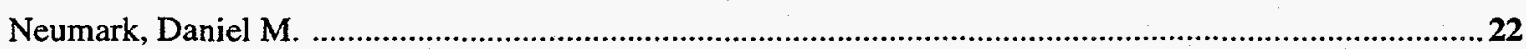

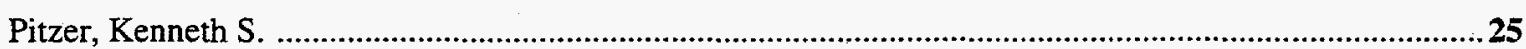

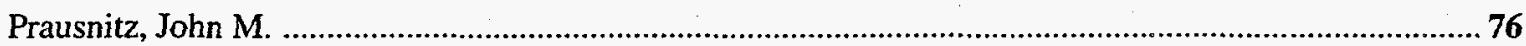

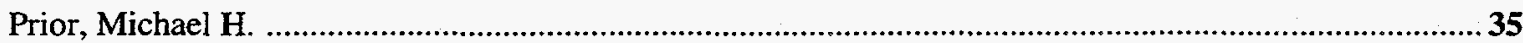

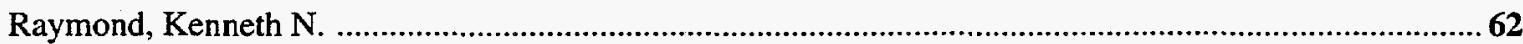

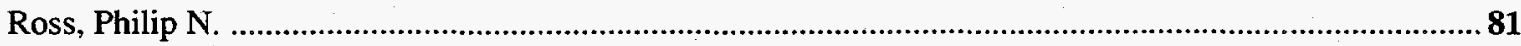

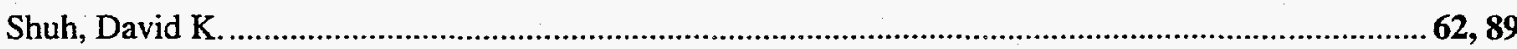

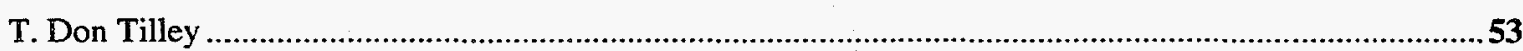

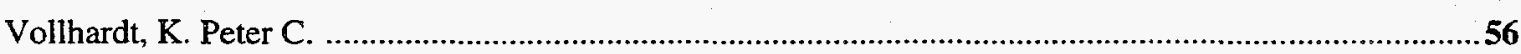

*Boldface numbers indicate investigators' main programs. 